(C)Copyright 2014

Maxwell T. Hansen 



\title{
Multi-Hadron Observables from Lattice Quantum Chromodynamics
}

\author{
Maxwell T. Hansen \\ A dissertation \\ submitted in partial fulfillment of the \\ requirements for the degree of \\ Doctor of Philosophy \\ University of Washington \\ 2014 \\ Reading Committee: \\ Stephen R. Sharpe, Chair \\ Ann Nelson \\ Andreas Karch
}

Program Authorized to Offer Degree:

Department of Physics 

University of Washington

\author{
Abstract \\ Multi-Hadron Observables from Lattice Quantum Chromodynamics \\ Maxwell T. Hansen \\ Chair of the Supervisory Committee: \\ Professor Stephen R. Sharpe \\ Department of Physics
}

We describe formal work that relates the finite-volume spectrum in a quantum field theory to scattering and decay amplitudes. This is of particular relevance to numerical calculations performed using Lattice Quantum Chromodynamics (LQCD). Correlators calculated using LQCD can only be determined on the Euclidean time axis. For this reason the standard method of determining scattering amplitudes via the Lehmann-Symanzik-Zimmermann reduction formula cannot be employed. By contrast, the finite-volume spectrum is directly accessible in LQCD calculations. Formalism for relating the spectrum to physical scattering observables is thus highly desirable.

In this thesis we develop tools for extracting physical information from LQCD for four types of observables. First we analyze systems with multiple, strongly-coupled two-scalar channels. Here we accommodate both identical and nonidentical scalars, and in the latter case allow for degenerate as well as nondegenerate particle masses. Using relativistic field theory, and summing to all orders in perturbation theory, we derive a result relating the finite-volume spectrum to the two-to-two scattering amplitudes of the coupled-channel theory. This generalizes the formalism of Martin Lüscher for the case of single-channel scattering. Second we consider the weak decay of a single particle into multiple, coupled two-scalar channels. We show how the finite-volume matrix element extracted in LQCD is related to matrix elements of asymptotic two-particle states, and thus to decay amplitudes. This generalizes work by Laurent Lellouch and Martin Lüscher. Third we extend 
the method for extracting matrix elements by considering currents which insert energy, momentum and angular momentum. This allows one to extract transition matrix elements and form factors from LQCD. Finally we look beyond two-particle systems to those with three-particles in asymptotic states. Working again to all orders in relativistic field theory, we derive a relation between the spectrum and an infinite-volume three-to-three scattering quantity. This final analysis is the most complicated of the four, because the all-orders summation is more difficult for this system, and also because a number of new technical issues arise in analyzing the contributing diagrams. 


\section{TABLE OF CONTENTS}

Page

List of Figures $\ldots \ldots \ldots \ldots \ldots \ldots \ldots \ldots$. . . . . . . . . . . . . . . . . . .

List of Tables . . . . . . . . . . . . . . . . . . . . . . . v

Chapter 1: Introduction . . . . . . . . . . . . . . . . . . . 1

1.1 Brief history of the Standard Model . . . . . . . . . . . . . . . . . 2

1.2 Brief history of Quantum Chromodynamics . . . . . . . . . . . . . . 5

1.3 Detailed description of QCD . . . . . . . . . . . . . . . . . . 8

1.4 Multi-hadron physics within and beyond the Standard Model . . . . . . . . . 11

1.5 Euclidean time $\ldots \ldots \ldots \ldots \ldots$

1.6 Finite volume . . . . . . . . . . . . . . . . . . . . . . . . . . . . . . . . . . . 28

Chapter 2: Scattering of Multiple Two-Particle Channels . . . . . . . . . . . . . 33

2.1 Multiple-channel extension of quantization condition . . . . . . . . . . . . 33

$2.2 \quad$ Multiple-channel quantization condition for s-wave scattering . . . . . . . . . 43

Chapter 3: Weak Decays into Multiple Two-Particle Channels . . . . . . . . . . 49

$3.1 \quad$ Multiple-channel extension of the Lellouch-Lüscher formula . . . . . . . . . . 49

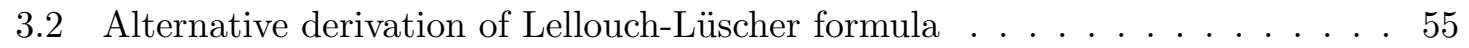

Chapter 4: One-to-Two Transition Matrix Elements . . . . . . . . . . . . . . . 61

4.1 Two-point correlation functions . . . . . . . . . . . . . . . . . . . . 65

4.2 Three-point correlation functions and the generalized Lellouch-Lüscher formula . . . . . . . . . . . . . . 88

Chapter 5: Three-Particle Scattering . . . . . . . . . . . . . . 108

5.1 Quantization Condition ． . . . . . . . . . . . . . . . . . . 110

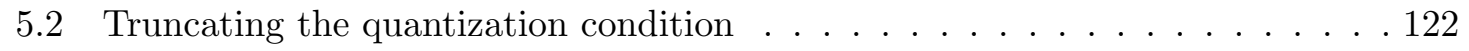

5.3 Derivation . . . . . . . . . . . . . . . . . . . . . 126

5.4 Conclusion of three-particle analysis $\ldots \ldots \ldots 4$ 
Chapter 6: Conclusion . . . . . . . . . . . . . . . . . . 195

Bibliography . . . . . . . . . . . . . . . . . . 196

Appendix A: Two-channel Watson's theorem . . . . . . . . . . . . . . . . 215

Appendix B: Cancelation of free poles . . . . . . . . . . . . . . . . . . . 218

Appendix C: $\quad$ Generalization for twisted boundary conditions in asymmetric volumes 222

Appendix D: Sum-minus-integral identity . . . . . . . . . . . . . . . . . . . 224

Appendix E: $\quad$ Smoothness of $\widetilde{\mathrm{PV}}$ pole prescription $\ldots \ldots \ldots \ldots \ldots$

Appendix F: Detailed study of the isotropic approximation . . . . . . . . . . 235 


\section{LIST OF FIGURES}

Figure Number $\quad$ Page

$1.1 \quad K \rightarrow \pi \pi$ Feynman diagrams . . . . . . . . . . . . . . . . . . . . 14

1.2 Summary of Minkowski vs. Euclidean correlators and transforms . . . . . . . 26

2.1 Definitions of (a) $C_{L}$, (b) $A$ and (c) $i \mathcal{M}$ and (d) subtracted correlator result . 36

$3.1 \quad$ Weak shift to scattering amplitude . . . . . . . . . . . . . . . . . 50

4.1 Definition of (a) two-particle correlator, (b) BS kernel, $K^{*}$, and (c) fully-

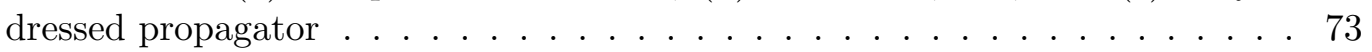

$4.2 \quad$ Definition of (a) scattering amplitude and (b) K-matrix . . . . . . . . . . . . 79

4.3 Definition of (a) three-point correlator and (b) BS kernel with external current 92

4.4 Definitions of transition amplitudes: (a) $\mathcal{A}$ and (b) $\mathbb{H}$. . . . . . . . . . . . 96

5.1 Three-to-three diagram with physical singularity . . . . . . . . . . . . . 115

5.2 Definition of divergence-free three-to-three amplitude, $\mathcal{M}_{\mathrm{df}, 3} \ldots \ldots \ldots$

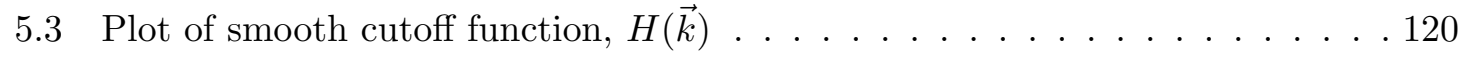

5.4 Skeleton expansion for finite-volume three-to-three correlator . . . . . . . . . 131

$5.5 \quad$ Examples of diagrams contributing to (a) $i B_{2}$ and $(\mathrm{b}) i B_{3} \ldots$. . . . . . . . 132

5.6 Definition of no-insertion finite-volume correlator, $C_{L}^{(0)} \ldots$. . . . . . . . . 134

5.7 Definition of no-switch finite-volume correlator, $C_{L}^{(1)} \ldots \ldots$. . . . . . . . . . . . . . . . . . . . . . . . . .

5.8 Finite-volume residue identity . . . . . . . . . . . . . . . . . . . 140

$5.9 \quad$ Diagrammatic reduction of $C_{L}^{(1)} \ldots \ldots \ldots \ldots \ldots$

5.10 Definition of one-switch finite-volume correlator, $C_{L}^{(2)} \ldots \ldots$. . . . . . . 148

5.11 Definitions of (a) $i \mathcal{K}_{3}^{(2, u, u)}$, (b) $A_{L}^{(2, u)}$ and (c) $C_{L, 0 F}^{(2)} \ldots \ldots \ldots . . . \ldots 151$

5.12 Diagrammatic decomposition of $\mathcal{K}_{3}^{(2, u, u)} \ldots \ldots \ldots \ldots$. . . . . . . . . . . . . . . . . . . . . . . . . . . . . . . .

5.13 Definitions of $(\mathrm{a}) A^{\prime(1, s)}$ and $(\mathrm{b}) A^{\prime(1)} \ldots \ldots \ldots$. . . . . . . . . . 161

5.14 Definition of two-switch finite-volume correlator, $C_{L}^{(3)} \ldots \ldots$. . . . . . 163

5.15 Definitions of $(\mathrm{a}) A_{L}^{\prime(3, u)}$, (b) $C_{L, 0 F}^{(3)}$ and $(\mathrm{c}) \mathcal{K}_{3, L}^{(3, u, u)} \ldots \ldots \ldots . . \ldots 165$

5.16 Diagrammatic decomposition of $\mathcal{K}_{3, L}^{(3, u, u)} \ldots \ldots \ldots$. . . . . . . . . 168

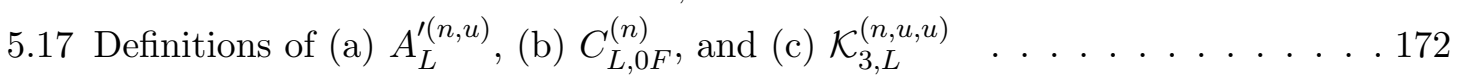


5.18 Diagrammatic decomposition of $\mathcal{K}_{3, L}^{(4, u, u)} \ldots \ldots \ldots \ldots$. . . . . . . 179

B.1 Definition of two-to-two correlation function . . . . . . . . . . . . . . . . . . 221

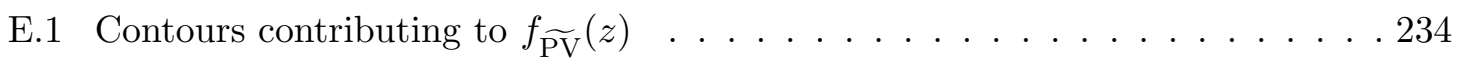

E.2 Comparision of $f_{\widetilde{\mathrm{PV}}}(x)$ and $f_{\mathrm{PV}}(x) \ldots \ldots \ldots \ldots \ldots \ldots \ldots \ldots \ldots \ldots \ldots$ 


\section{LIST OF TABLES}

Table Number $\quad$ Page

$1.1 \quad$ Basic properties of SM quarks . . . . . . . . . . . . . . . . . . . . . . 9

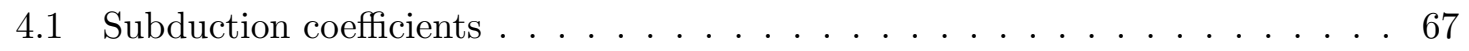

$4.2 \quad$ Nonzero values of $\alpha_{20, \Lambda}^{\mathrm{d}}$ and $\alpha_{22, \Lambda}^{\mathrm{d}}$ for $\mathbf{d}^{2} \leq 3 \ldots \ldots \ldots \ldots$ 


\section{ACKNOWLEDGMENTS}

I would like to thank my advisor, Steve Sharpe. I was so excited when Steve and I started working on the project that became my first paper. We were working together on the hardest physics problem I had ever pursued, and I felt like an equal collaborator. Looking back, I am convinced that Steve could have pushed that project to completion much more quickly, and that he showed masterful restraint to let me chip away until the result started to unfold. While I was buried in the challenge of calculating, Steve was focused on the more complicated and long-term project of building me into an independent researcher. I am very grateful that I managed to stumble into such incredibly competent hands.

Next I would like to thank my other collaborators in work presented here, Raúl Briceño and André Walker-Loud. These gentlemen have contributed significantly to my understanding of physics, and also to my understanding of the physics community in which we work. Raúl has showed me what real passion for the subject looks like, a constant development of new ideas and a powerful drive to organize and refine understanding at every opportunity. André has taught me to think big.

I thank Ruth Van de Water, Andreas Kronfeld and everyone else that I met at Fermilab, for the year I spent working with and learning from them. The opportunity to work in an entirely different environment with such strong physicists was much appreciated. I thank my reading committee, Ann Nelson and Andreas Karch, and the rest of the particle/nuclear theory group. For discussion and camaraderie I am grateful to Seyda, John, Han, Bridget, Stefan, Jakub, Zohreh and the rest of the particle theory grads/postdocs, to all my physics buddies in my year and to the greater UW physics community.

I close here by acknowledging all of the wonderful people in my life outside of physics. I have so many people in my life that give me such joy, and without them this work would never have been completed. In particular I want to mention everyone in 5249, my oldest 
friends back in Bozeman, my family near and extended.

Finally, I acknowledge Esther, who is so very important to me. I am excited about the years to come. 


\section{DEDICATION}

to Mom, Dad and Simon 


\section{Chapter 1}

\section{INTRODUCTION}

In this thesis we present formalism that allows one to extract scattering and decay amplitudes from numerical calculations of quantum chromodynamics (QCD). QCD is the accepted underlying theory of the strong interaction and it contains very rich phenomenlogy but also poses great theoretical challenges. Here we consider the goal of extracting physical predictions from lattice QCD (LQCD), which is a regularization of the theory that makes numerical calculation possible. We analyze systems with multiple strongly interacting particles (hadrons) in the initial or final states. In fact, the relations that we present in this thesis provide the only known method to study such processes quantitatively, with systematically improvable uncertainties. The formalism thus offers important opportunities to better understand the predictions of QCD and, by comparing predictions to experimental results, to investigate new physics models.

To fully explain the applications of this work, we begin by establishing the currently accepted underlying theory of our universe, the Standard Model (SM). In Section 1.1 we summarize the Standard Model by describing the history of its construction. We then review the history of QCD, our main focus within the SM, in Section 1.2 . In Section 1.3 we give a detailed, quantitative discussion of QCD. Next, to better motivate our interest, in Section 1.4 we describe a number of specific processes that can be studied using the results we derive. With these established we turn in Sections 1.5 and 1.6 to two technical aspects of numerical LQCD that are central to the work of this thesis. These are Euclidean time (1.5) and finite volume (1.6). We also summarize our main results in Section 1.6. 


\subsection{Brief history of the Standard Mode 1}

In 1897, Sir Joseph John (J.J.) Thomson demonstrated that cathode rays are comprised of charged particles with mass over one thousand times smaller than that of the hydrogen atom [2, 3]. Twenty years later, Ernest Rutherford found the particle that makes up the rest of hydrogen's mass. By examining a nuclear reaction between nitrogen and alpha particles (helium nuclei), he discovered a postively charged particle with mass very close to the hydrogen atom, which he named the proton. It was soon clear that the proton and electron alone could not account for all of the known elements. The next heaviest element, helium, was found to have a nucleus with twice the proton charge, but roughly four times the mass. This observation was explained with the discovery of the neutron, a neutral twin of the proton, by Sir James Chadwick in 1932 [4. Simultaneous with this work, the particle nature of light was developed from 1900 to 1924, primarily by Max Planck, Albert Einstein, and Arthur Compton [5, 6, 7, 8, 9]. Thus, by 1932, a beautifully simple picture of matter and light had emerged, up to a few exceptions that we now describe.

The first important complication was the prediction of antiparticles by Paul Dirac. These emerged as solutions to a relativistic wave equation that Dirac had found in 1927 [10, 11. In 1931 Carl Anderson discovered the anti-electron or positron, confirming the bold prediction [12. This lead to the new understanding that every particle has an antiparticle with opposite charge $2^{2}$ In a very short period the number of fundamental building blocks was nearly doubled and the puzzle of matter/anti-matter asymmetry was born.

Another complication appeared in 1930 with the discovery of the neutrino. Looking at the beta decay of various nuclei, Wolfgang Pauli and others recognized that the energy distribution was not consistent with the two visible decay products. Pauli posited that a third invisible particle must be carrying away the missing energy. A more complete and predictive theory of beta decay was then provided by Enrico Fermi, who named the missing particle the neutrino [13, 14].

Within a few years the story broadened yet again with the prediction by Hideki Yukawa

\footnotetext{
${ }^{1}$ This account is drawn heavily from Ref. [1]

${ }^{2}$ Certain particles with zero charge, such as the photon, turn out to be their own antiparticles.
} 
of a new type of particle called a meson. The meson was motivated by the question of what holds nuclei together. For example, the two positively charged protons in a helium nucleus would repel in the absence of some short-ranged attractive strong force. That the electromagnetic force is mediated by the photon was already well established, so it was natural that Yukawa should look for some strong-force mediating particle. He further found that the mass of the mediator was dictated by the size of nuclei to be nearly 300 times that of the electron [15].

In 1937 two separate groups, Anderson and Neddermeyer on the west coast of the United States and Street and Stevenson on the east, identified Yukawa's particle in cosmic rays [16, 17, 18. However, these investigations were plagued by some confusion. The problem, as was finally settled in 1947 by Cecil Powell and collaborators, was the existence of two different types of particles in cosmic rays [19, 20]. One is the meson predicted by Yukawa, now called the pi meson or pion, the other is a heavier cousin of the electron, called the muon, which has no direct relation to the strong force. For a brief period in this year, it was possible to believe that all fundamental particles had been discovered.

The illusion of completeness did not last long. In December of 1947 Rochester and Butler published a cloud chamber photograph that showed a heavy neutral particle which had decayed into two oppositely charged pions [21]. The new particle was named the Kmeson, later shortened to kaon. This began an explosion of discovery, with dozens of new particles appearing in the 1950s as collider and detector technology improved. The newfound abundance completely disrupted the simple organized picture. In 1955, in his Nobel Prize acceptance speech, Willis Lamb joked that "the finder of a new elementary particle used to be rewarded by a Nobel Prize, but such a discovery now ought to be punished by a $\$ 10,000$ fine." [22] This multitude of particles was explained in the 1960s and 1970s with the development of the quantum theory of the strong force. We discuss this work in some detail in the next section and do not pursue it further here.

The theory of the electromagnetic and weak interactions reached its modern form slightly before that of the strong force. First quantum electrodynamics (QED), the quantum theory governing the interaction of charged particles via photon exchange, was developed. Its main architects included Hans Bethe, Freeman Dyson, Sin-Itiro Tomonaga, Julian Schwinger and 
Richard Feynman [23, 24, 25, 26, 27, 28, 29, 30, 31]. The framework, which had remarkable predictive power and would become the standard for all future quantum field theories, was largely complete within a few years of the pion and the kaon discoveries, by 1950. Over a decade later in 1961, Sheldon Glashow combined the then well-established QED with the weak interaction into a single unified theory [32]. Six years after this, Steven Weinberg and Abdus Salam incorporated the newly formulated Higgs mechanism into Glashow's framework, thereby reaching the modern theory of the electroweak interaction [33, 34]. Thus, already by 1967, the electroweak part of the Standard Model was fully in place. The completion of the strong interactions were roughly half a decade behind.

The Higgs mechanism within the electroweak sector resolved certain theoretical issues and in doing so predicted an additional particle, a spin-zero scalar called the Higgs boson [35, 36, 37]. The experimental verifcation of this new particle was a long way off and would be preceeded by the discovery of three other bosons in the theory, the massive vector particles that mediated the interaction, called $W^{+}, W^{-}$and $Z$. The mass of these vectors gave the weak interaction a short range, just as with the strong interaction and Yukawa's meson. In contrast to the strong interaction however, no weak bound states were available to set the scale for the exchange particles.

But the theory of Glashow, Weinberg and Salam provided a firm prediction for the weak vector masses, with the mass landing around 100 times that of the proton. The construction of a collider to find the weak vector bosons began at CERN in the late 1970s and culminated in 1983 with the discovery by Carlo Rubbia's group of both the charged and neutral vectors [38, 39]. At this point the Standard Model was on firm ground, awaiting discoveries of only three more particles, with the most prominent being the unique fundamental spin-zero boson called the Higgs.

The saga ends nearly three decades later. At this point all Standard Model particles had been found except for the immensely important Higgs boson. Searches for the elusive particle had begun at CERN in the 1990s and had continued at Fermilab through 2011, when the collider there was shut down. In this period plans were devised and pushed forward at CERN to build a Large Hadon Collider (LHC), with primary focus being the discovery of the final missing piece. The gargantuan effort achieved first data collection in March 2010. 
The LHC continued to take data over the next two years and finally, on July 4th of 2012, the ATLAS and CMS collaborations announced in a joint press conference the discovery of a Higgs-like boson with mass over 130 times that of the proton [40, 41].

With this achievement the Standard Model was completed, some 115 years after the discovery of its first fundamental building block.

\subsection{Brief history of Quantum Chromodynamics}

As mentioned above, experiments in the 1950s showed that a large number of new particles, collectively called hadrons, are produced in proton and electron collisions. Drawing on earlier work from Eugene Wigner and Werner Heisenberg, Kazuhiko Nishijima, Tadao Nakano and Murray Gell-Mann discovered how to organize this "particle zoo", classifying the new particles according to electric charge and isospin as well as a new property denoted strangeness [42, 43, 44, 45, 46]. In 1963, Gell-Mann and George Zweig independently proposed that the classification could be efficiently explained by introducing smaller consitutients inside the hadrons, spin-1/2 fermions called quarks [47, 48, 49] ? $^{3}$ At that time three different kinds, or flavors, were needed. These were named up, down and strange.

Shortly after Gell-Mann and Zweig's papers, in a footnote in a 1965 preprint, Boris Struminsky noted that the $\Omega^{-}$hyperon must contain three quarks of the same flavor (strange) and the same spin. However, being identical fermions, quarks are required to be in a state that is anti-symmetric under particle exchange. Struminsky argued that this anti-symmetry could only be accomplished by introducing a new quantum number [51].

Similar observations were made in the same year by Moo-Young Han with Yoichiro Nambu as well as independently by Oscar W. Greenberg [52, 55]. These groups proposed

\footnotetext{
${ }^{3}$ Zweig preferred the term "ace" for the particle he proposed, but it was Gell-Mann's terminology that became widely accepted. Gell-Mann also said later that he was undecided on the spelling of the new word, until he found a passage in James Joyce's Finnegans Wake [50]:
}

Three quarks for Muster Mark!

Sure he has not got much of a bark

And sure any he has it's all beside the mark. 
that quarks possess an additional degree of freedom, called color, and that each quark flavor forms a triplet of color. In more technical language, Han and Nambu as well as Greenberg suggested that quarks transform as the three-dimensional (fundamental) representation of an $S U(3)$ gauge group 4 The mathematical structure of such a theory had already been developed over a decade earlier by Chen Ning Yang and Robert Mills [54]. In a gauge theory, additional vector particles called gauge bosons are automatically included. These particles transform as the adjoint of the gauge group, which is eight-dimensional in the present case, corresponding to an octet of particles known as gluons. Gluons mediate the quark interactions and are responsible for holding quarks together inside hadrons. This quantum theory of quarks and gluons, which is known as Quantum Chromodynamics (QCD), is the modern accepted theory of the strong interaction.

Although the quantum numbers of the three lightest quarks were understood by 1965 , the physics community was still divided about whether the constituents should be understood as an organizational tool or as fundamental particles. The central concern was that no experiments had shown quarks in isolation, and these should have been easy to identify since they were predicted to have fractional electric charge. In 1968 James Bjorken proposed certain relations in deep inelastic scattering of electrons and protons which, if seen experimentally, would confirm that the protons were composed of pointlike constituents [55]. These were verified by experiments at SLAC in 1969 and provided strong evidence for a particle interpretation of quarks [56, 57].

Nevertheless, in the years that followed the community was still far from unified about the nature of the constituents. The absence of isolated quarks in experiment, as well as the apparently ad hoc implementation of color to avoid the Pauli exclusion principal, left many particle physicists uneasy. This doubt was largely resolved in what came to be known as the November Revolution, a new meson discovery that was announced in November of 1974 [58, 59]. The new meson had actually already been seen by C. C. Ting and collaborators in the Summer of 1974. However Ting wanted to check the results before announcing them,

\footnotetext{
${ }^{4} S U(3)$ is the group formed by matrix multiplication on set of all complex three-by-three matrices $U$ which satisfy $U U^{\dagger}=1$ and $\operatorname{det} U=1$.
} 
and so the discovery was kept quiet until Burton Richter's group at SLAC also saw the particle. Ting named the particle $J$ and Richter called it $\psi$, and so the modern designation contains both these labels. The $J / \psi$ is a neutral meson, which was heavier than any that had been seen before. Particularly striking was that the state has a very long lifetime compared with others of similar mass. These observations were explained by identifying the $J / \psi$ as a bound state of a new quark, called charm, together with a charm anti-quark. The introduction of the charm quark provided a rich set of predictions that were later confirmed, and for most, this breakthrough firmly established the existence of quarks. Indeed, two still heavier quarks have since been discovered: the bottom quark in 1977 and the top quark in 1995, both at Fermilab 60, 61, 62.

The heavier quark discoveries were particularly significant, because the properties of mesons built from heavier quarks are easier to predict. To explain this fact, we now present the final crucial step toward the modern understanding of the strong interaction. This is the discovery of asymptotic freedom by David Gross and Frank Wilczek and independently by David Politzer, in 1973 [63, 64]. In their Nobel Prize winning works, Gross, Politzer and Wilczek showed that the coupling constant which governs the strength of the strong interaction decreases with increasing energy. Indeed, the early 1970s were a crucial time for developing technical understanding of QCD. In the year before Gerard 't Hooft and Martinus Veltman proved that QCD was renormalizable, meaning that the theory was calculable to any fixed order in perturbation theory [65.5 Asymptotic freedom and renormalizability imply that perturbative analysis is both rigorous and predictive, but only for high energy observables. For example, heavier-quark mesons are more amenable to perturbative analysis than those built from up, down and strange. Experiments at PETRA in 1979 found clear evidence for the validity of this perturbative understanding, and the approach was confirmed at the few percent level with the LEP experiments at CERN [66, 67].

The flipside of asymptotic freedom is that the strength of the coupling constant increases with decreasing energy. This makes plausible, but does not prove, that interactions at

\footnotetext{
${ }^{5}$ Interestingly Gerardus 't Hooft understood the scaling of QCD a year before the publications of Gross, Politzer and Wilczek, and apparently deemed it unworthy of publication.
} 
low energies are so strong that the relevant degrees of freedom change. More precisely, if the interactions are strong enough, then the energy cost to isolate quarks produces new quark anti-quark pairs out of the vacuum. In this description, the interquark potential is expected to increase linearly with separation, like the potential of a taut string. It follows that energy rises indefinitely with separation until the string is broken by a new particle/antiparticle pair. This idea is called confinement. It explains why quarks are never seen in isolation, because such a state would cost infinite energy. Although no formal proof exists, the evidence by now is overwhelming.

\subsection{Detailed description of $Q C D^{6}$}

We now completely specify the modern theory of the strong force, QCD. We do not directly use the results of this section anywhere below and only include it for completeness.

We begin by specifying the quark-field-dependent terms of the QCD Lagrangian density

$$
\mathcal{L}_{q}=-\bar{\Psi}_{i, x}\left(-i \not \phi_{i j}+m_{x} \delta_{i j}\right) \Psi_{j, x}
$$

where $i, j$ are color indices running from 1 to 3 and $x$ is a flavor index running from 1 to 6 . (In many cases the sum runs only up to 2,3 , or 4 , as the heavier quarks can be neglected. The quark flavors of the standard model are summarized in Table 1.1.) Each $\Psi_{i, x}$ field is a four-component Dirac spinor, with Dirac indices suppressed.

Here we have also introduced

$$
\not D_{i j}=\gamma^{\mu} D_{\mu, i j}=\gamma^{\mu}\left[\partial_{\mu} \delta_{i j}-i g A_{\mu}^{a} T_{i j}^{a}\right]
$$

where $\gamma^{\mu}$ is a Dirac matrix satisfying $\left[\gamma^{\mu}, \gamma^{\nu}\right]=-2 g^{\mu \nu}$ with $g^{\mu \nu}$ the mostly-plus Minkowski metric, $p_{\mu} p_{\nu} g^{\mu \nu}=-\left[p^{0}\right]^{2}+\mathbf{p}^{2}$. The second term in Eq. 1.2 results in a quark-quark-gluon coupling. It consists of the gluon field $A_{\mu}^{a}$, the generator matrix $T_{i j}^{a}$ and the strong coupling constant $g$. Here $a$ runs over the 8 generators of $S U(3)$ and $T_{i j}^{a}$ is understood to be in the fundamental representation.

It remains only to specify the pure-glue or Yang-Mills part of the theory

$$
\mathcal{L}_{\mathrm{YM}}=-\frac{1}{4} F^{c \mu \nu} F_{\mu \nu}^{c}
$$

\footnotetext{
${ }^{6}$ Conventions in this section are taken from Ref. 68.
} 


\begin{tabular}{c|cccccc} 
& up & down & strange & charm & bottom & top \\
\hline \hline \multirow{2}{*}{ mass $(\mathrm{MeV})$} & $2.3_{-0.5}^{+0.7}$ & $4.8_{-0.3}^{+0.5}$ & $95 \pm 5$ & $1,275 \pm 25$ & $4,180 \pm 30$ & $160,000_{-4,000}^{+5,000}$ \\
& & & & & & \\
charge & $+2 / 3$ & $-1 / 3$ & $-1 / 3$ & $+2 / 3$ & $-1 / 3$ & $+2 / 3$
\end{tabular}

Table 1.1: Basic properties of the six quarks in the Standard Model. All masses are in $\overline{\mathrm{MS}}$ renormalization scheme. For the lightest three the renormalization scale is $\mu=2 \mathrm{GeV}$ and for the heaviest three the scale is equal to the particle mass [69].

where

$$
F_{\mu \nu}^{c}=\partial_{\mu} A_{\nu}^{c}-\partial_{\nu} A_{\mu}^{c}+g f^{a b c} A_{\mu}^{a} A_{\nu}^{b}
$$

Note that this part of the Lagrangian density gives three and four-gluon vertices. This self interaction of the exchange particles is the central feature that distinguishes a non-abelian gauge theory from an abelian gauge theory.

The total Lagrangian density is thus $\mathcal{L}=\mathcal{L}_{q}+\mathcal{L}_{\mathrm{YM}}$ and the action is $S=\int d^{4} x \mathcal{L}$. From this one can formally determine any correlator using the path-integral relation

$$
\left\langle 0\left|T \hat{\mathcal{O}}_{n}\left(x_{n}\right) \cdots \hat{\mathcal{O}}_{1}\left(x_{1}\right)\right| 0\right\rangle=\frac{1}{Z} \int \mathcal{D} A \mathcal{D} \Psi \mathcal{D} \bar{\Psi} \exp [i S] \mathcal{O}_{n}\left(x_{n}\right) \cdots \mathcal{O}_{1}\left(x_{1}\right)
$$

where $T$ denotes time ordering and

$$
Z \equiv \int \mathcal{D} A \mathcal{D} \Psi \mathcal{D} \bar{\Psi} \exp [i S]
$$

It is not, however, a priori clear that this path-integral is well-defined. One way to make sense of the integral is in perturbation theory. As we have already mentioned, it is now well established that the theory is calculable at any fixed order in perturbation theory. To perform such calculations one must gauge-fix and then regularize and renormalize ultraviolet divergences. Certain quantities also suffer from infrared divergences but these cancel in any physical observable. Perturbative QCD is not relevant to the present work and we therefore make no further mention of this extensive field. 
Another tool for understanding QCD is to consider a modified theory in which spacetime is discretized. By this we mean that quark fields are restricted to only take values on a lattice of points

$$
\psi(x) \longrightarrow \psi(a n)
$$

where $a$ is the lattice spacing and $n$ is a four-vector of integers. Additional work is needed to completely define and make sense of the discretized theory. We do not describe the steps here but merely assert that it is well understood how to define a discretized path integral and then how to perform a continuum limit to recover desired physical quantities. Discretizing QCD is the only known way to regulate ultraviolet divergences without first performing a perturbative expansion. Since perturbation theory does not describe the lowenergy properties of the theory, the availability of a nonperturbative regulator is crucial.

Although important progress has been made via analytic studies of LQCD, the bulk of modern focus is on numerical calculations of the theory. This requires restricting the discretized theory to a finite (usually periodic/antiperiodic) spacetime, so that the path integral reduces to a finite-dimensional integral. In addition, because the integration space is so large, numerical work relies on Monte Carlo sampling to approximate the desired result. This technique only applies when the integrand is positive definite, and thus cannot be used with the complex oscillatory factor, $\exp [i S]$. For this reason the numerical path-integral must be performed in Euclidean rather than Minkowski field theory.

In this work we consider the role of Euclidean time and finite volume in extracting physical observables from LQCD. In particular, we are interested in processes that have two or more on-shell hadrons in the initial or final state 7 Before describing how LQCD

\footnotetext{
${ }^{7}$ As we describe in more detail in the finite-volume section below, extracting observables is often easier when only one (or zero) hadrons appears in the initial or final state. For example, matrix elements of local operators between single-particle states can be directly extracted by taking an appropriate limit on Euclidean time coordinates. This approach is already being widely used in precision numerical studies. I am currently working with Jack Laiho and Ruth S. Van de Water to extract matrix elements relevant for neutral kaon mixing. These matrix elements predict the rate at which a kaon oscillates into an anti-kaon. Our study considers operators both within and beyond the Standard Model, and comparison with experiment will thus provide constraints on new physics models.
} 
can be used to study such processes we present a series of specific examples to motivate our interest.

\subsection{Multi-hadron physics within and beyond the Standard Model}

In this section we discuss various decay and scattering processes that have multiple hadrons in the initial/final state. The specific cases that we consider highlight the significance of the work presented in the following chapters. However, it is important to emphasize that the examples are only illustrative, and that the formalism that we present below is much more general. In particular, in this thesis we derive formalism to extract the following quantities from numerical LQCD:

1. two-to-two scattering amplitudes, for scalar particles in systems with any number of coupled two-particle channels,

2. weak decay matrix elements, with a two-scalar final state that is coupled to other two-particle channels,

3. one-to-two scalar transition matrix elements, for currents that inject energy, momentum and angular momentum,

4. three-to-three scattering quantities, for identical scalar particles with no other open scattering channels.

Numerical LQCD is the only known first-principles approach for predicting these quantities from the underlying theory. Since a method for extracting these observables was unavailable before this work, we think the results are an important asset in reaching a detailed, quantitative understanding of the strong interaction.

The rest of this section is organized as follows. In Subsection 1.4.1 we discuss the decay $K \rightarrow \pi \pi$. The formalism for determining this decay rate from LQCD was developed by Martin Lüscher and Laurent Lellouch in the (very good) approximation that the lightest two quarks have equal mass [70. In Subsection 1.4.2 we discuss various phenomenologically interesting two-particle resonances including $a_{0}(980)$ and $f_{0}(980)$. These can be 
systematically studied with our coupled two-scalar scattering formalism (1 above). Next in Subsection 1.4 .3 we consider $D \rightarrow \pi \pi, K \bar{K}$, which is accessible via our weak decay relation (2), in the approximation where coupling to higher particle states is neglected. In Subsection 1.4.4 we discuss various semi-leptonic/photonic processes such as such as $\pi \gamma \rightarrow \pi \pi$. These are accessible from lattice calculations via our formal developments for transition matrix elements (3). Finally in Subsection 1.4.5 we discuss three particle resonances including $N(1440) \rightarrow N \pi \pi$ and $\omega(782) \rightarrow \pi \pi \pi$. Our three-particle analysis (4) represents progress towards first principles analysis of these states.

\section{$1.4 .1 \quad K \rightarrow \pi \pi$}

Mesons with the quantum numbers of one strange antiquark and one of the lightest two quarks are called kaons. These particles have a rich phenomenology and provide important opportunities for testing and constraining physics that goes beyond the Standard Model (BSM physics). Because strangeness is conserved in QCD, kaons can only decay through the weak interactions. Decays can be leptonic as well as semileptonic, involving both leptons and hadrons, and purely hadronic. The last of these provides an opportunity to explore the interplay of weak and strong interactions, with incoming and outgoing QCD states coupled only via charged weak vectors, $W^{ \pm}$. An old problem in this sector, specifically in $K \rightarrow \pi \pi$ decays, is the $\Delta I=1 / 2$ rule. This "rule" is the experimental observation, made nearly sixty years ago, that kaons (which have isospin of one-half) decay to the isospin-zero state with a much higher rate than to the isospin-two state [71, 72, 173. The enhancement is measured to be 74

$$
\left.\frac{\operatorname{Re}\left[A_{K} \longrightarrow(\pi \pi, I=0)\right]}{\operatorname{Re}\left[A_{K} \longrightarrow(\pi \pi, I=2)\right.}\right]=22.2 \pm 0.1
$$

Here $A$ is a decay amplitude and so the ratio of rates is larger, equal to the ratio of magnitudes squared.

The $\Delta I=1 / 2$ rule predates $\mathrm{QCD}$, and early attempts to understand the observation were completely unsuccessful. Evaluating matrix elements of

$$
\left[\bar{s} \gamma^{\mu}\left(1-\gamma_{5}\right) u\right]\left[\bar{u} \gamma_{\mu}\left(1-\gamma_{5}\right) d\right]
$$


within the quark-model, one finds that the ratio of amplitudes should be order one, in stark tension with experiment [74].

Understanding was greatly improved with the advent of QCD and the operator product expansion. Using these tools, the decay amplitudes can be determined from matrix elements of a low-energy effective hamiltonian [75, 76, 77, 78, 79]

$$
\mathcal{H}_{\mathrm{eff}}^{\Delta S=1}=\frac{G_{F}}{\sqrt{2}} \sum_{i=1}^{8}\left[\left(V_{u s}^{*} V_{u d}\right) z_{i}(\mu)-\left(V_{t s}^{*} V_{t d}\right) y_{i}(\mu)\right] Q_{i}(\mu) \quad\left(\mu<m_{c}\right)
$$

where $Q_{i}(\mu)$ are four quark operators and $z_{i}(\mu)$ and $y_{i}(\mu)$ are Wilson coefficients. The latter contain information on scales above the renormalization scale $\mu$, and for $\mathrm{CP}$ conserving processes only the $z_{i}(\mu)$ are numerically relevant. Here we have also introduced the Fermi decay constant $G_{F}=1.16637(1) \times 10^{-5} \mathrm{GeV}^{-2}$ as well as certain elements of the CabibboKobayashi-Maskawa (CKM) matrix, $V_{x y}$ [69].

The Wilson coefficients can be calculated perturbatively for $\mu \gtrsim 1 \mathrm{GeV}$. Indeed major progress in the understanding of the $\Delta I=1 / 2$ rule was made when it was observed that the quark evolution, represented by the Wilson coefficients, leads to both an enhancement of the $I=0$ and a suppression of the $I=2$ final state [75, 76, 80, 81, 82, 83]. Nevertheless, perturbative effects are not sufficient to fully explain Eq. (1.8) and the low energy QCD matrix elements are needed.

We do not display the explicit forms of the four-quark operators $Q_{i}(\mu)$ here, but only comment that these include that of Eq. (1.9), which is due to $W^{+}$exchange [see Figure 1.4.1(a)], as well as penguin operators such as [74]

$$
\sum_{q}\left[\bar{s} \gamma^{\mu}\left(1-\gamma_{5}\right) d\right]\left[\bar{q} \gamma_{\mu}\left(1-\gamma_{5}\right) q\right], \quad \sum_{q}\left[\bar{s} \gamma^{\mu}\left(1-\gamma_{5}\right) q\right]\left[\bar{q} \gamma_{\mu}\left(1-\gamma_{5}\right) d\right]
$$

where the sum runs over up, down and strange. These operators arise from so-called penguin diagrams like that shown in Figure 1.4.1(b).

To extract $K \rightarrow \pi \pi$ amplitudes, one must determine

$$
\left\langle\pi \pi, I\left|Q_{i}(\mu)\right| K^{0}\right\rangle
$$

These matrix elements must be calculated using non-perturbative techniques, with the only 


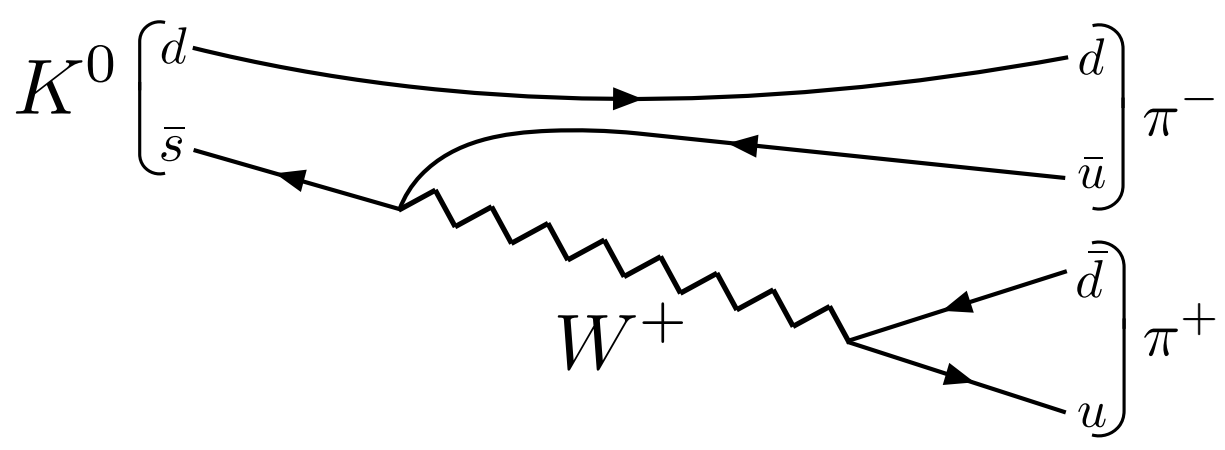

$(a)$

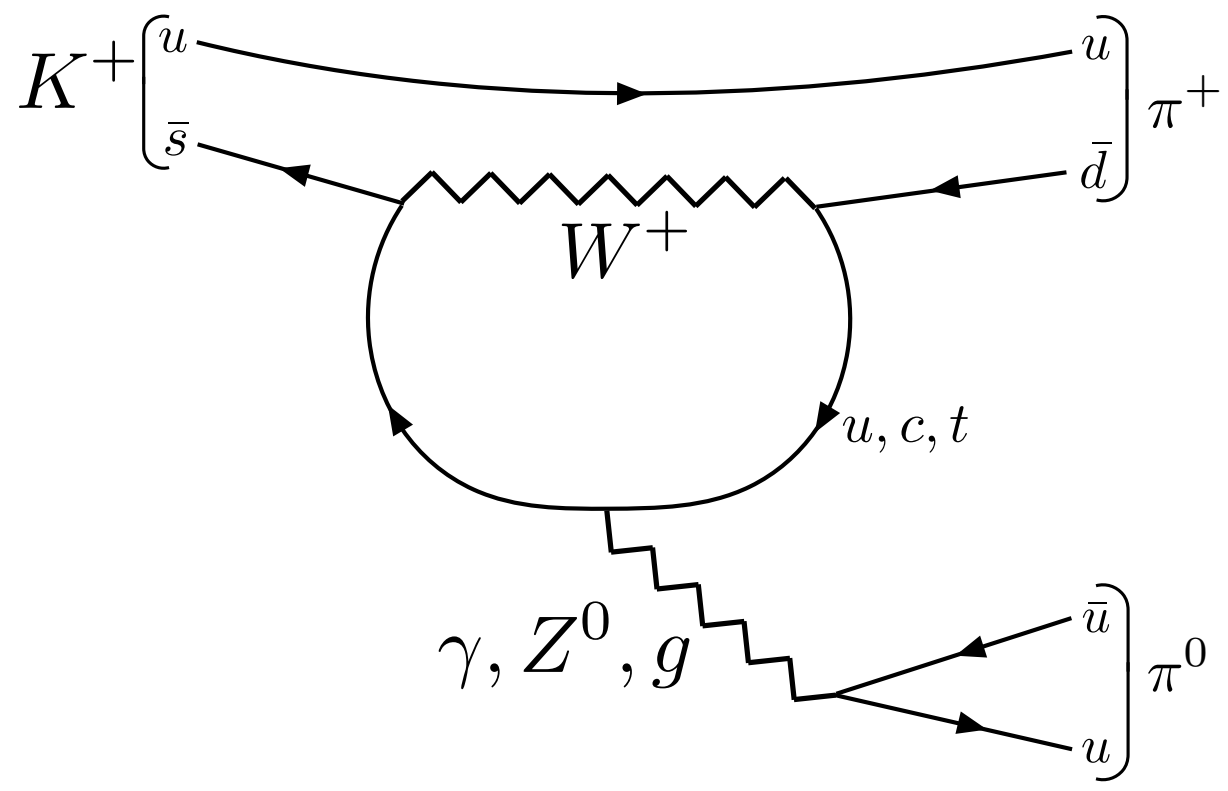

(b)

Figure 1.1: Feynman diagrams responsible for $K \rightarrow \pi \pi$ : (a) tree-level W-exchange diagram (b) one-loop penguin diagram. 
known sytematically improvable option being $\mathrm{LQCD}^{8} \mathrm{~A}$ full-error-budget calculation is now being implemented by the RBC/UKQCD collaboration [88, 89, 90]. The methodology is fully in place, results for the $I=2$ final state with a complete error budget are available [91], and complete results for the more challenging $I=0$ final states should become available in the next few years. At that stage we will finally learn whether and in what manner QCD can explain the $\Delta I=1 / 2$ rule and the observed CP-violation 9 rate in $K \rightarrow \pi \pi$ decays.

\subsubsection{Two-particle resonances}

The rich resonance structure observed in hadronic scattering experiments is expected to be completely described by QCD. Here we consider the energy regime where only twoparticle channels are open. The scattering amplitudes connecting these channels contain all information about the two-particle resonances of the theory.

More precisely, the eigenstates of the QCD hamiltonian are asymptotic states built only from those mesons and baryons that are stable in the absence of the electroweak interaction. All predictions of the theory are contained within the scattering matrix (S-matrix) which is the matrix of overlaps between all possible incoming and outgoing asymptotic states. In the two-particle sector, the S-matrix may be expressed in the space of open two-particle channels together with angular momentum which, at fixed energy, completely specifies the states. The simplest case is a single two-particle channel, for which the matrix reduces to a single phase for each value of angular momentum, $S_{J}=\exp \left[2 i \delta_{J}\right]$. A resonance is defined as a sharp increase of the phase-shift $\delta_{J}$ as a function of the two-particle center of mass (CM) frame energy. A more rigorous definition is possible if one knows the analytic form of $S$ in the complex CM-energy plane. Then a resonance is defined as a pole located off the real axis.

In Chapter 2 we present a method for extracting the S-matrix from LQCD, for a system with multiple strongly-coupled two-scalar channels. By extracting scattering observables at

\footnotetext{
${ }^{8}$ Other options include the vacuum saturation approximation, which fails completely, and the large $N_{c}$ (number of colors) expansion, which predicts the correct order of magnitude for the decay rates [77, 84, 85, 86. 87.

${ }^{9} \mathrm{C}$ is charge conjugation symmetry, $\mathrm{P}$ is parity.
} 
many energies and fitting the result to a well-motivated analytic function, one can use this method to determine $S$ off the real axis. The poles in the result then give the full resonance structure of the theory.

This method has already been applied in a full LQCD calculation, at unphysically heavy $391 \mathrm{MeV}$ pion mass, to the $I=1 / 2 \pi K, \eta K$ system [92.10 This study, which considers $J^{P}=0^{+}, 1^{-}, 2^{+}$, demonstrates that the formalism can be applied to physically relevant systems and that resonance properties can be rigorously extracted. In particular, the authors find a narrow scalar resonance and a broad tensor resonance resembling the $K_{0}^{*}(1430)$ and the $K_{2}^{*}(1430)$ respectively. In the $J=1^{-}$channel they find a bound state below $\pi K$ threshold. This is expected to become the experimentally known narrow resonance at physical pion masses.

Many other states could be investigated using this approach. For example, QCD phenomenologists are interested in better understanding two scalar resonances with energies close to the $K \bar{K}$ threshold, known as the $a_{0}(980)(I=1)$ and the $f_{0}(980)(I=0)$. These are sufficiently narrow that it seems well-motivated to associate them with certain excited states of the quark model. However they do not fit into the scalar $q \bar{q}$ nonet, and thus other solutions are required. Possible alternatives include interpreting the narrow resonances as tetraquark states or as $K \bar{K}$ molecules [93, 94, 95, 96, 97, 98]. In general these are modeldependent descriptions, but in certain contexts a more rigorous definition can be applied. In this sector much insight could be gained by using LQCD to extract resonance pole positions, as well as to determine overlaps of various operators with scattering states at the pole.

\subsection{3 $D \rightarrow \pi \pi, K \bar{K}$}

In December of 2011 the $\mathrm{LHCb}$ experiment at CERN reported evidence for CP-violation in (the difference of) $D^{0} \rightarrow \pi^{+} \pi^{-}$and $D^{0} \rightarrow K^{+} K^{-}$decays [99]. Although the initially reported rate was larger than naive expectations from the Standard Model, there was sufficient uncertainty in the Standard Model prediction for it to be consistent with the result

\footnotetext{
${ }^{10}$ Note that the $\eta$ is a resonance for physical pion mass but is a stable particle for the pions used in this calculation.
} 
(see, e.g. Refs. [100, 101, 102, 103, 104, 105]). The violation was then reduced in a more complete analysis from May of 2014 [106]. Nevertheless, it is interesting to understand whether a calculation of such decays using lattice methods is feasible.

In the context of LQCD the issue turns out to be a generic field-theoretic question of whether decays into multiple, strongly-coupled channels can be analyzed. In Chapter 3 we demonstrate that this is possible and detail the method for decay into coupled two-scalar channels. This formalism allows one to analyze $D \rightarrow \pi \pi, K \bar{K}$ in the approximation where coupling to higher-particle states is neglected. This can likely only offer a rough guide of the physical decay rates. A qualitative indication of this (ignoring differences in phase space) is that the $f_{0}(1500)$ has a $50 \%$ branching fraction to $\pi \pi \pi \pi$, while the branches to $\pi \pi, K \bar{K}$ and $\eta \eta$ are $\sim 35 \%, 9 \%$ and $5 \%$, respectively [69].

\subsubsection{One-to-two transition matrix elements}

A LQCD calculation of proton-proton fusion through the weak interaction, $p p \rightarrow d e^{+} \nu_{e}$, would allow for a direct theoretical prediction of this fundamental process which powers the sun. The MuSun Collaboration will measure a related process, muon capture on deuterium [107]. At low energies, these two processes are described by the same two-nucleon contact interaction [108, providing an opportunity to over-constrain these reactions for which there is currently discrepancy between experimental results [109, 110] and theory calculations [108, 111].

Another example of particular interest is the heavy meson decay $B^{0} \rightarrow K^{*} \ell^{+} \ell^{-} \rightarrow$ $\pi K \ell^{+} \ell^{-}$which could be used to probe physics beyond the Standard Model. Also for this process there is currently tentative tension between experimental results [112, 113, 114, 115, 116] and Standard Model predictions [117, 118, 119, 120].

In Chapter 4 we demonstrate how to unambiguously study $\mathbf{1} \rightarrow \mathbf{2}$ form factors via lattice QCD. Specifically our formalism allows one to extract matrix elements of energy/angularmomentum injecting currents between a one-particle state and a two-particle asymptotic state. This is a first step towards constructing a formalism for the determination of $\mathbf{2} \rightarrow \mathbf{2}$ form factors, which is directly relevant for $p p \rightarrow d e^{+} \nu_{e}$. 
$1.4 .5 \quad N(1440) \rightarrow N \pi \pi, \omega(782) \rightarrow \pi \pi \pi, K_{2}^{*}(1430) \rightarrow K \pi \pi$

A better understanding of the Roper resonance $N(1440)$ has been an important goal of QCD phenomenologists for decades. It is unnatural from the perspective of quark models, since its mass is lower than that of the negative-parity ground state $N(1535)$ [121, 122].

As with the other states discussed, a quantitative prediction of the $N(1440)$ properties from first principles QCD in only possible via LQCD. Also as above, the formalism to extract the resonance's properties from the underlying theory is currently unavailable. In the present work we take an important step towards this formalism. Specifically, in Chapter 5 , we analyze a single scalar particle with no coupling between odd and even particle states. Within this set-up we derive a relation between the three-particle finite-volume spectrum and a particular (non-standard) infinite-volume quantity. Our formalism is strictly valid for three pion states in QCD and could be used to investigate the $\omega(782)$ resonance, which decays to three pions 11

Future work will generalize this to accommodate non-identical and non-degenerate scalar particles and also particles with spin. This will allow one to treat the Roper resonance and also $K_{2}^{*}(1430) \rightarrow K \pi \pi$. This is well-motivated by the current status of LQCD calculations. In particular, the aforementioned LQCD calculation of $\pi K, \pi \eta$ scattering was limited, in the $J=2^{+}$sector, by the opening of the $K^{*} \rightarrow K \pi \pi$ threshold [92]. Our formalism for this system could thus potentially allow lattice practitioners to extract physical information from existing numerical data.

\footnotetext{
${ }^{11}$ For physical pion masses the resonance would also couple to five-pion states, invalidating the analysis presented here. This issue would potentially be evaded in simulations with unphsyically heavy pions. Here one must determine what range of pion masses gives a resonant $\omega(782)$ which only decays to three-pion states. Practically one would likely proceed to analyze the three-particle spectrum at some pion mass without knowing the phenomenology. If the extracted resonance sits between three and five particle masses then the concluded properties are formally rigorous. If the resonance exceeds the five particle threshold, then the result must be interpreted with a systematic uncertainty due to the neglected on-shell state.
} 


\subsection{Euclidean time}

Having discussed a number of interesting processes with two or more hadrons in the initial or final states, we now return to the task of understanding how these can be studied using LQCD. As mentioned above, this thesis emphasizes the role of Euclidean time and finite volume in extracting multi-hadron observables. In this section we carefully discuss the former.

We first recall the main motivation: Numerical lattice QCD calculations can only be performed using Euclidean time. This is because only Euclidean path integrals have a real positive integrand that can be evaluated using Monte Carlo techniques. For this reason, it is paramount to understand what information can be extracted from numerically determined correlators with Euclidean time coordinates. In the following subsection we carefully define the Euclidean correlator. Then, in Subsection 1.5.2, we discuss the difficulties of extracting physical scattering information from these objects.

\subsubsection{Definition of the Euclidean correlator}

We begin with the Minkowski two-point correlator

$$
\left\langle 0\left|\hat{\mathcal{O}}_{2}\left(x^{0}, \mathbf{x}\right) \hat{\mathcal{O}}_{1}\left(y^{0}, \mathbf{y}\right)\right| 0\right\rangle=\frac{1}{Z} \int \mathcal{D} A \mathcal{D} \Psi \mathcal{D} \bar{\Psi} \exp [i S] \mathcal{O}_{2}\left(x^{0}, \mathbf{x}\right) \mathcal{O}_{1}\left(y^{0}, \mathbf{y}\right)
$$

where we have assumed $x^{0}>y^{0}$. Restricting attention to the left-hand side, we insert a complete set of states and use the identity for time translation of quantum operators to reach

$$
\begin{aligned}
& \left\langle 0\left|\hat{\mathcal{O}}_{2}\left(x^{0}, \mathbf{x}\right) \hat{\mathcal{O}}_{1}\left(y^{0}, \mathbf{y}\right)\right| 0\right\rangle= \\
& \sum_{n} \int \frac{d^{3} \mathbf{P}}{(2 \pi)^{3} 2 E_{n, \mathbf{P}}} \exp \left[-i E_{n, \mathbf{P}}\left(x^{0}-y^{0}\right)\right]\left\langle 0\left|\hat{\mathcal{O}}_{2}(0, \mathbf{x})\right| n, \mathbf{P}\right\rangle\left\langle n, \mathbf{P}\left|\hat{\mathcal{O}}_{1}(0, \mathbf{y})\right| 0\right\rangle
\end{aligned}
$$

Here we label eigenstates of the Hamiltonian by their total momentum $\mathbf{P}$ as well as by a collective label $n$, which encodes all other properties. We stress that $n$ might also include continuous degrees of freedom, so that the schematic sum may also contain integrals. We also assume throughout that the ground state is unique and has zero energy. 
If the integrals in Eq. 1.14 are convergent, then it is clear that extending $x^{0}-y^{0}$ into the negative complex plane will only improve this convergence. In particular, if we rotate to pure imaginary values of the same magnitude, denoted $-i\left(x_{4}-y_{4}\right)$, then the relation becomes

$$
\begin{aligned}
& \left\langle 0\left|\hat{\mathcal{O}}_{E, 2}\left(x_{4}, \mathbf{x}\right) \hat{\mathcal{O}}_{E, 1}\left(y_{4}, \mathbf{y}\right)\right| 0\right\rangle \equiv \\
& \quad \sum_{n} \int \frac{d^{3} \mathbf{P}}{(2 \pi)^{3} 2 E_{n, \mathbf{P}}} \exp \left[-E_{n, \mathbf{P}}\left(x_{4}-y_{4}\right)\right]\left\langle 0\left|\hat{\mathcal{O}}_{2}(0, \mathbf{x})\right| n, \mathbf{P}\right\rangle\left\langle n, \mathbf{P}\left|\hat{\mathcal{O}}_{1}(0, \mathbf{y})\right| 0\right\rangle .
\end{aligned}
$$

This is the definition of the Euclidean correlator.

We next claim that this Euclidean correlator, and its generalization to any number of fields, is defined by a modified path integral. To review the derivation of the path-integral formula, we do not work within QCD but instead consider a one-dimensional, real scalar field theory. This captures the basic points of the derivation but avoids a number of complicating details.

We begin by expressing the time dependence via the time evolution operator, but in contrast to Eq. 1.15, we do so without inserting a complete set of states. Assuming $x_{4}>y_{4}$, we find

$$
\begin{aligned}
\left\langle 0\left|\hat{\mathcal{O}}_{E, 2}\left(x_{4}\right) \hat{\mathcal{O}}_{E, 1}\left(y_{4}\right)\right| 0\right\rangle & =\left\langle 0\left|\hat{\mathcal{O}}_{E, 2}(0) \exp \left[-\hat{H}\left(x_{4}-y_{4}\right)\right] \hat{\mathcal{O}}_{E, 1}(0)\right| 0\right\rangle \\
=\frac{1}{Z^{\prime}} & \lim _{\left(T_{+} \rightarrow+\infty, T_{-} \rightarrow-\infty\right)} \int d \phi \int d \phi^{\prime} \\
& \times\left\langle\phi^{\prime}\left|e^{-\hat{H}\left(T_{+}-x_{4}\right)} \hat{\mathcal{O}}_{E, 2}(0) e^{-\hat{H}\left(x_{4}-y_{4}\right)} \hat{\mathcal{O}}_{E, 1}(0) e^{-\hat{H}\left(y_{4}-T_{-}\right)}\right| \phi\right\rangle,
\end{aligned}
$$

where we have introduced

$$
Z^{\prime} \equiv \lim _{\left(T_{+} \rightarrow+\infty, T_{-} \rightarrow-\infty\right)} \int d \phi \int d \phi^{\prime}\left\langle\phi^{\prime}\left|\exp \left[-\hat{H}\left(T_{+}-T_{-}\right)\right]\right| \phi\right\rangle
$$

Here $|\phi\rangle$ is an eigenstate of our one-dimensional field $\hat{\phi}(0)$ with eigenvalue $\phi$. To see that Eq. 1.17 holds, note that the integrals over eigenstates of $\hat{\phi}(0)$ can be rewritten as linear combinations of Hamiltonian eigenstates. The exponential factors then remove all excited states and the $1 / Z^{\prime}$ pre-factor cancels the coefficients on the two vacuum states.

The next step is to write each time evolution operator as a product of $N \gg 1$ factors 
which each evolve by an infinitesimal amount. For example

$$
\begin{gathered}
a=b \\
c=d \\
\exp \left[-\hat{H}\left(x_{4}-y_{4}\right)\right]=\exp [-\hat{H} \epsilon] \exp [-\hat{H} \epsilon] \times \cdots \times \exp [-\hat{H} \epsilon],
\end{gathered}
$$

where $N_{m}$ factors appear on the right-hand side and $\epsilon \equiv\left(x_{4}-y_{4}\right) / N_{m}$. We then insert a complete set of states

$$
1=\int d \phi|\phi\rangle\langle\phi|
$$

between each pair of adjacent infinitesimal time-translators. This gives a large number of factors with the form

$$
\left\langle\phi_{\tau+1}\left|e^{-\hat{H} \epsilon}\right| \phi_{\tau}\right\rangle
$$

where $\tau$ is an integer labeling the various variables of integration.

If we assume the Hamiltonian takes the standard form $\hat{H}=\hat{\Pi}^{2} / 2+V(\hat{\phi})$ then this factor can be further simplified

$$
\begin{aligned}
\left\langle\phi_{\tau+1}\left|e^{-\hat{H} \epsilon}\right| \phi_{\tau}\right\rangle & =\int \frac{d \Pi}{2 \pi}\left\langle\phi_{\tau+1} \mid \Pi\right\rangle\left\langle\Pi\left|e^{-\epsilon \hat{\Pi}^{2} / 2} e^{-\epsilon V(\hat{\phi})}\right| \phi_{\tau}\right\rangle+\mathcal{O}\left(\epsilon^{2}\right), \\
& =C \exp \left[-\epsilon\left\{\frac{1}{2} \frac{\left(\phi_{\tau+1}-\phi_{\tau}\right)^{2}}{\epsilon^{2}}+V\left(\phi_{\tau}\right)\right\}\right]+\mathcal{O}\left(\epsilon^{2}\right) .
\end{aligned}
$$

Absorbing all factors of $C$ into $Z=Z^{\prime} / C^{N}$ and dropping $\mathcal{O}\left(\epsilon^{2}\right)$ terms, we deduce

$$
\left\langle 0\left|\hat{\mathcal{O}}_{E, 2}\left(x_{4}\right) \hat{\mathcal{O}}_{E, 1}\left(y_{4}\right)\right| 0\right\rangle=\frac{1}{Z} \int D \phi \exp \left[-S_{E}\right] \mathcal{O}\left(x_{4}\right) \mathcal{O}\left(y_{4}\right)
$$

where

$$
\begin{aligned}
\int D \phi & \equiv\left[\prod_{\tau=N_{-}}^{N_{+}} \int d \phi_{\tau}\right] \\
S_{E} & \equiv \epsilon \sum_{\tau=N_{-}}^{N_{+}} \mathcal{L}_{E}(\tau) \equiv \epsilon \sum_{\tau=N_{-}}^{N_{+}}\left[\frac{1}{2} \frac{\left(\phi_{\tau+1}-\phi_{\tau}\right)^{2}}{\epsilon^{2}}+V\left(\phi_{\tau}\right)\right] \\
\mathcal{O}(\tau) & \equiv\left\langle\phi_{\tau+1}|\hat{\mathcal{O}}(0)| \phi_{\tau}\right\rangle
\end{aligned}
$$

and

$$
Z \equiv \int D \phi \exp \left[-S_{E}\right]
$$


Finally, if we take the limit in which the time slices become arbitrarily close together $(\epsilon \rightarrow 0)$ then Eq. 1.28 becomes

$$
S_{E} \equiv \int_{-\infty}^{\infty} d \tau \mathcal{L}_{E}(\tau) \equiv \int_{-\infty}^{\infty} d \tau\left[\frac{1}{2}\left[\partial_{\tau} \phi(\tau)\right]^{2}+V[\phi(\tau)]\right]
$$

Actually, this limit is highly subtle, since it involves approaching an infinite continuum of integration variables. We will not pursue this issue further here but merely assert that practitioners of LQCD are concerned with taking the (closely related) continuum limit in order to extract physical observables. Although there is no proof that the limit is welldefined, numerical implementation has been highly successful.

Next observe that the quantity $S_{E}$, in Eq. 1.31, is a modified form of the action that defines the real scalar field theory. In particular, it is reached by rotating the time coordinate from the real to the imaginary axis, and then relabeling. This is also the correct prescription to identify the Euclidean path-integral of QCD, one rotates from $\left(x^{0}, \mathbf{x}\right)$ to real $\left(x_{4}, \mathbf{x}\right)$, where $x_{4}=i x^{0}$

$$
\begin{aligned}
i S & =i \int d x^{0} \int d \mathbf{x} \mathcal{L}\left(x^{0}, \mathbf{x}\right) \\
& =\int_{\mathcal{I}} d x_{4} \int d \mathbf{x} \mathcal{L}\left(-i x_{4}, \mathbf{x}\right) \\
& \longrightarrow \int_{\mathcal{R}} d x_{4} \int d \mathbf{x} \mathcal{L}\left(-i x_{4}, \mathbf{x}\right) \equiv-\int d^{4} x_{E} \mathcal{L}_{E}\left(x_{E}\right) \equiv-S_{E} .
\end{aligned}
$$

To go from Eq. 1.32 to 1.33 , we have simply relabeled $x^{0}=-i x_{4}$. The subscript $\mathcal{I}$ indicates that integration is along the imaginary $x_{4}$ and therefore real $x^{0}$ axis. Here the first two lines are trivially equivalent and only involve relabeling. To go from Eq. 1.33 to (1.34), we rotate the integration contour to the real $x_{4}$ axis. Thus, in this step the action changes from a functional of fields on real $x^{0}$ to a functional of fields on real $x_{4}$.

Returning to notation that is appropriate for QCD we reach

$$
\begin{aligned}
\left\langle 0\left|T \mathcal{O}_{E, n}\left(x_{E, n}\right) \cdots \mathcal{O}_{E, 1}\left(x_{E, 1}\right)\right| 0\right\rangle & \\
& =\frac{1}{Z} \int \mathcal{D} A_{E} \mathcal{D} \Psi_{E} \mathcal{D} \bar{\Psi}_{E} \exp \left[-S_{E}\right] \mathcal{O}_{E, n}\left(x_{E, n}\right) \cdots \mathcal{O}_{E, 1}\left(x_{E, 1}\right)
\end{aligned}
$$

where $T$ denotes time ordering and

$$
Z \equiv \int \mathcal{D} A_{E} \mathcal{D} \Psi_{E} \mathcal{D} \bar{\Psi}_{E} \exp \left[-S_{E}\right]
$$


As already mentioned, the discretized version of this path-integral can be numerically evaluated. We now consider how the Euclidean time signature limits the information that one can extract.

\subsubsection{Extracting Minkowski correlators from infinite-volume Euclidean correlators}

Because numerical lattice calculations are performed in a finite volume and with nonzero lattice spacing, the Euclidean correlators that one extracts will differ from those of the infinite-volume continuum theory. However, it is at least in principle possible to run calculations with many different lattice and box sizes, and so to systematically reduce these effects. With this in mind, we now consider what information can be extracted from numerically determined, infinite-volume/continuum correlation functions, evaluated at Euclidean time. We will find that physical multi-hadron observables cannot be determined in this way, and this will motivate the remainder of this work, which relies on finite-volume as a tool to overcome the obstacle.

The first natural question is whether it is possible to convert

$$
\left\langle 0\left|T \mathcal{O}_{E, n}\left(x_{E, n}\right) \cdots \mathcal{O}_{E, 1}\left(x_{E, 1}\right)\right| 0\right\rangle_{\text {num }} \longrightarrow\left\langle 0\left|T \mathcal{O}_{n}\left(x_{n}\right) \cdots \mathcal{O}_{1}\left(x_{1}\right)\right| 0\right\rangle_{\text {num }}
$$

where the subscript indicates that the functions are known numerically. This represents an ill-posed problem in most cases of interest [123, and the issue has been quantititatively investigated in the context of inverse Laplace transforms [124]. To understand the connection, it is neccesary to first introduce the momentum-space Euclidean correlator

$$
\begin{aligned}
G_{E}\left(q_{E, 1}, \cdots,\right. & \left.q_{E, n}\right) \equiv \int d^{4} x_{E, 1} \cdots d^{4} x_{E, n} \exp \left[-i\left(x_{1,4} q_{1,4}+\cdots+x_{n, 4} q_{n, 4}\right)\right] \\
& \times \exp \left[-i\left(\mathbf{x}_{1} \cdot \mathbf{q}_{1}+\cdots+\mathbf{x}_{n} \cdot \mathbf{q}_{n}\right)\right]\left\langle 0\left|T \mathcal{O}_{E, n}\left(x_{E, n}\right) \cdots \mathcal{O}_{E, 1}\left(x_{E, 1}\right)\right| 0\right\rangle
\end{aligned}
$$

Next observe that $G_{E}$ can be written as the Laplace transform of the position-space Minkowski correlator. This is achieved by rotating the $x_{4}$ integrals from the real to the imaginary axis. Here one must take care to rotate the contours in the direction that gives damping at infinity. For example, considering the case when all $q_{4}<0$, we split all $x_{4}$ integrals into the positive and negative halves of the real axis. For the term with all positive ranges we rotate 
to the positive imaginary axis to find

$$
\begin{aligned}
& G_{E}\left(q_{E, 1}, \cdots, q_{E, n}\right)=i^{n} \int_{x^{0}=0}^{x^{0}=\infty} d^{4} x_{1} \cdots d^{4} x_{n} \exp \left[-x_{1}^{0}\left|q_{1,4}\right|-\cdots-x_{n}^{0}\left|q_{n, 4}\right|\right] \\
& \times \exp \left[-i\left(\mathbf{x}_{1} \cdot \mathbf{q}_{1}+\cdots+\mathbf{x}_{n} \cdot \mathbf{q}_{n}\right)\right]\left\langle 0\left|T \mathcal{O}_{n}\left(x_{n}\right) \cdots \mathcal{O}_{1}\left(x_{1}\right)\right| 0\right\rangle+\cdots
\end{aligned}
$$

where $\cdots$ indicates a series of similar terms that arrise from integrals over the negative real axis. We generally expect the Laplace transform to converge, since the Minkowksi correlator has oscillatory time dependence, so the integrand above decays exponentially with time. We thus deduce that the problem of analytic continuation may be reexpressed as that of finding the inverse Laplace transform

$$
G_{E}\left(q_{E, 1}, \cdots, q_{E, n}\right)_{\text {num }} \quad \underset{\text { inverse } \mathrm{LT}}{\longrightarrow} \quad\left\langle 0\left|T \mathcal{O}_{n}\left(x_{n}\right) \cdots \mathcal{O}_{1}\left(x_{1}\right)\right| 0\right\rangle_{\text {num }}
$$

Ref. [124] discusses in great detail why this problem is "severely ill posed". The authors first point out that, while the Laplace transform is continuous, its inverse is not. The argument introduces a sequence of functions $f_{n}\left(x^{0}\right)$, with $n=1,2,3, \cdots$, together with their Laplace transforms $g_{n}\left(q_{4}\right)$. The authors demonstrate that it is possible to construct a sequence such that as $n \rightarrow \infty$

$$
\int_{0}^{\infty} d q_{4}\left|g_{n}\left(q_{4}\right)\right|^{2} \longrightarrow 0 \quad \text { while } \quad \int_{0}^{\infty} d x^{0}\left|f_{n}\left(x^{0}\right)\right|^{2} \longrightarrow \infty
$$

To see the significance of this, we introduce $G\left(q_{4}\right)$ as the exact Laplace transform, and observe that $G\left(q_{4}\right)_{\text {num }, n} \equiv G\left(q_{4}\right)+g_{n}\left(q_{4}\right)$ defines a sequence of approximations that improve with increasing $n$. Specifically, the approximations improve in the sense that the norm of $G\left(q_{4}\right)_{\text {num }, n}-G\left(q_{4}\right)$ vanishes with increasing $n$. (Recall that the norm squared is defined as the integral of the function's magnitude squared, as given in Eq. (1.41.) Remarkably, however, the difference between the exact inverse $F\left(x^{0}\right)$ and the approximate inverses $F\left(x^{0}\right)_{\text {num }, n} \equiv F\left(x^{0}\right)+f_{n}\left(x^{0}\right)$ has divergent norm in the limit $n \rightarrow \infty$.

The situtation is improved if we include the assumption that $F\left(x^{0}\right)$ must be a continuous function. In this case the approximate inverse Laplace transform will at least formally approach the exact result. However if the norm of $G\left(q_{4}\right)-G\left(q_{4}\right)_{\text {num }, n}$ is bounded by some vanishing $\epsilon$, then the norm of $F\left(x^{0}\right)-F\left(x^{0}\right)_{\text {num }, n}$ will only vanish as $[\log (\epsilon)]^{-1}$. Thus, the errors in the inverse are significantly enhanced. 
Abandoning the approach of direct analytic continuation, progress can be made by recalling that we are often interested in the momentum-space Minkowski correlator. This is defined as

$$
\begin{aligned}
G\left(q_{1}, \cdots, q_{n}\right) \equiv \int d^{4} x_{1} \cdots d^{4} x_{n} \exp \left[i\left(x_{1}^{0} q_{1}^{0}+\cdots+x_{n}^{0} q_{n}^{0}\right)\right] \\
\quad \times \exp \left[-i\left(\mathbf{x}_{1} \cdot \mathbf{q}_{1}+\cdots+\mathbf{x}_{n} \cdot \mathbf{q}_{n}\right)\right]\left\langle 0\left|T \mathcal{O}_{n}\left(x_{n}\right) \cdots \mathcal{O}_{1}\left(x_{1}\right)\right| 0\right\rangle
\end{aligned}
$$

We thus consider whether it is justified to rotate the integration and so deduce

$$
\begin{aligned}
G\left(q_{1}, \cdots, q_{n}\right) & \stackrel{?}{=}(-i)^{n} \int d^{4} x_{E, 1} \cdots d^{4} x_{E, n} \exp \left[x_{1,4} q_{1}^{0}+\cdots+x_{n, 4} q_{n}^{0}\right] \\
& \times \exp \left[-i\left(\mathbf{x}_{1} \cdot \mathbf{q}_{1}+\cdots+\mathbf{x}_{n} \cdot \mathbf{q}_{n}\right)\right]\left\langle 0\left|T \mathcal{O}_{E, n}\left(x_{E, n}\right) \cdots \mathcal{O}_{E, 1}\left(x_{E, 1}\right)\right| 0\right\rangle
\end{aligned}
$$

This is closely related to the preceeding analysis. The difference is that we are working here with the momentum-space Minkowski correlator rather than the momentum-space Euclidean correlator. In Figure 1.2 we summarize the four types of correlators and the relationships between them. Note that the Laplace transform is used to change between position and momentum space while simultaneously changing between Euclidean and Minkowski time. It turns out that Laplace transforming the Euclidean position-space correlator to extract the Minkowski momentum-space correlator works for certain momenta ranges, but fails generally.

Consider, for example, the simplest possible case of a two point function in free scalar field theory. We slightly modify the definition given in Eq. (1.42), instead taking

$$
G(k) \equiv \int d^{4} x \exp \left[i\left(x^{0} k^{0}-\mathbf{x} \cdot \mathbf{k}\right)\right]\langle 0|T \phi(x) \phi(0)| 0\rangle=\frac{1}{i} \frac{1}{-\left[k^{0}\right]^{2}+\omega_{k}^{2}-i \epsilon},
$$

where we have introduced $\omega_{k} \equiv \sqrt{\mathbf{k}^{2}+m^{2}}$, with $m$ the mass of the scalar particle. Here only one Fourier transform was performed because the second simply generates a momentum conserving delta-function which plays no role in the present argument.

It is next useful to consider the expression at an intermediate stage when only the spatial integral has been evaluated

$$
G(k)=\frac{1}{2 \omega_{k}} \int d x^{0} \exp \left[i x^{0} k^{0}-i\left(\omega_{k}-i \epsilon\right)\left|x^{0}\right|\right] .
$$




$\begin{array}{cccc} & \begin{array}{c}\text { Minkowski } \\ \text { time }\end{array} & & \begin{array}{c}\text { Euclidean } \\ \text { time }\end{array} \\ \begin{array}{c}\text { momentum } \\ \text { space }\end{array} & G(p, \cdots) & \text { a.c. } & G_{E}\left(p_{E}, \cdots\right) \\ & \text { f.t. } & \text { l.t.? } & \text { f.t. } \\ \begin{array}{c}\text { position } \\ \text { space }\end{array} & \langle 0|\mathcal{O}(x) \cdots| 0\rangle & \text { a.c. } & \left\langle 0\left|\mathcal{O}_{E}\left(x_{E}\right) \cdots\right| 0\right\rangle\end{array}$

Figure 1.2: Summary of the various relations between Minkwoski/Euclidean time and position/momentum space correlators. The letters between the various correlator pairs indicate the relation: a.c. for analytic continuation, f.t. for Fourier transform, l.t. for Laplace transform. The question mark on l.t. emphasizes that the transform does not always correctly relate the correlators, as we discuss in the text. 
When the Euclidean spatial correlator in Eq. 1.43) is used to attempt the same result, one instead arrives at

$$
G(k) \stackrel{?}{=}-\frac{i}{2 \omega_{k}} \int d x_{4} \exp \left[x_{4} k^{0}-\omega_{k}\left|x_{4}\right|\right] .
$$

We deduce that Eq. 1.46) only produces the correct result for $\left[k^{0}\right]^{2}<\omega_{k}^{2}$. Outside of this region the Euclidean integral is divergent and therefore clearly gives no information about the true Minkowski propagator. The problem arises because the contour at infinity, which is implicitly neglected in going from Eq. 1.45 to Eq. 1.46) (more generally from 1.42) to 1.43 ), gives an important contribution once the magnitude of $k^{0}$ exceeds $\omega_{k}$. (Indeed the neglected contour gives a divergent contribution which, if correctly accounted for, must cancel the divergence in Eq. (1.46) to give the known result.)

In this simplest of cases the convergence issue can be resolved by splitting the range of integration as was done in Eq. (1.39) above, and then treating $\left[k^{0}\right]^{2}<\omega_{k}^{2}$ and $\left[k^{0}\right]^{2}>\omega_{k}^{2}$ separately. In this way one can reproduce the free Minkowski propagator, except for the $i \epsilon$ shift of the single-particle pole. However, this cumbersome approach does not generalize well to the more-complicated correlators of interacting theories. In particular, no implementation of Laplace transforms can give the correct result for an interacting theory at energies above multi-particle thresholds. The reason is that in this case the result is imaginary due to the integration (with $i \epsilon$ prescription) along the multi-particle branchcut. Since Eq. (1.43) is pure real, up to an overall phase, the correct result cannot be reproduced ${ }^{12}$ We direct the reader to Ref. [125] for additional discussion as well as a more physically interesting example where the Laplace transform is valid.

We conclude this section by offering a final attempt to extract physical information from numerically determined Euclidean correlation functions. Maiani and Testa studied infinite-volume Euclidean correlators of the form [126]

$$
G_{\mathbf{q}}\left(t_{1}, t_{2}\right)=\left\langle\pi_{\mathbf{q}}\left(t_{1}\right) \pi_{-\mathbf{q}}\left(t_{2}\right) J(0)\right\rangle
$$

where $J(0)$ is a current with the quantum numbers of two pions. They considered taking a large $t_{1}$ limit followed by a large $t_{2}$ limit. This would naively interpolate a two-pion out

\footnotetext{
${ }^{12}$ Looking to Eq. 1.43 we see that the integral is real as long as the correlator is invariant under $\mathbf{x}_{i} \rightarrow-\mathbf{x}_{i}$ for all $i$. This is satisfied as long as parity is a symmetry of the theory.
} 
state, but as one might expect from the preceding discussion, a more complicated structure emerges.

In a careful and thorough analysis, the authors found

$$
\begin{aligned}
& G_{\mathbf{q}}\left(t_{1}, t_{2}\right) \stackrel{t_{1} \gg t_{2} \gg 0}{\longrightarrow} \frac{Z_{\pi}}{2 \omega_{q}} \exp \left[-\omega_{q}\left(t_{1}+t_{2}\right)\right] \\
& \times\left[(1 / 2)\left(\left\langle\pi_{\mathbf{q}} \pi_{-\mathbf{q}}, \text { out }|J(0)| 0\right\rangle+\left\langle\pi_{\mathbf{q}} \pi_{-\mathbf{q}}, \text { in }|J(0)| 0\right\rangle\right)+C \exp \left[2\left(\omega_{q}-m_{\pi}\right) t_{2}\right]+\cdots\right],
\end{aligned}
$$

where $\cdots$ indicate terms that are suppressed in the large time limit. Observe that the first term in square brackets involves an average of in- and out-states, rather than the outstate alone. This is not so surprising since the correlator is known to be real and the average has this property. The average is not particularly problematic. For example, below the four-particle threshold the average could be used to determine the two-particle phase shift, which is the only physically relevant scattering information anyway.

This information is however unavailable, as a result of the second term in square brackets. Note that this term increases with increasing $t_{2}$ and thus dominates the on-shell matrix elements in the large time limit. The coefficient $C$ depends on off-shell matrix elements with no clear physical interpretation. Note that the second term is only growing for nonzero $\mathbf{q}$ and in fact has no $t_{2}$ dependence at threshold $(\mathbf{q}=\mathbf{0})$. Indeed, Maiani and Testa also considered the threshold limit of this result, and found that it does produce a useful result

$$
G_{\mathbf{0}}\left(t_{1}, t_{2}\right) \stackrel{t_{1} \gg t_{2} \gg 0}{\longrightarrow} \frac{Z_{\pi}}{2 m_{\pi}}\langle\pi|J(0)| \pi\rangle\left(1-a \sqrt{\frac{m_{\pi}}{4 \pi t_{2}}}+\cdots\right)
$$

where $a$ is the $\mathrm{S}$-wave scattering length.

In summary, in this section we have argued that no analytic continuation, in position or momentum space, is possible above two-particle threshold. We have further shown that taking asymptotic time limits directly on Euclidean correlators only provides scattering information at threshold. This would appear severely limiting for LQCD, but fortunately, as we describe in the next section, the problem can be circumvented.

\subsection{Finite volume}

Having argued that multi-hadron observables cannot be extracted from numerically determined, infinite-volume Euclidean correlators, we now consider the effects of finite-volume. 
We work throughout the remaining text with a finite periodic spatial-volume of linear extent $L$. We continue to treat the time direction as infinite. This is well motivated because finite-Euclidean-time (equivalently finite-temperature) effects are smaller in typical numerical calculations and also because the difficulties faced in infinite-volume are most easily overcome by only compactifying spatial directions.

The finite-volume theory has a discrete energy spectrum which varies with $L$. The discrete tower of states may be organized according to all quantum numbers which are conserved in finite-volume, and it is useful to separately consider the spectrum in each subspace. For concreteness consider states with the quantum numbers of two pions in a given isospin channel $(I=0,1,2)$ and with zero total momentum. We denote the spectrum in this subspace by $E_{1}(L), E_{2}(L), \cdots$. Two-pion states are disjoint from single and three-pion states because they have different eigenvalues under G-parity, which we treat as an exact symmetry here. We stress that quantum numbers are the only tool for classifying states, because we have no concept of asymptotic states to classify multi-particle configurations. For example two and four-particle states can only be separated kinematically, and we do so by restricting attention to $0<E<4 m_{\pi}$, with $m_{\pi}$ the physical pion mass. Observe next that, for any finite $n, E_{n}(L)$ approaches $2 m_{\pi}$ in the limit $L \rightarrow \infty$. Thus in this limit the spectrum contains no information about particle interactions. On the other hand, at finite $L$ the values of $E_{1}(L), E_{2}(L), \cdots$ must differ from those of two-free particles as a result of particle interactions. It follows that an expression which specifies the values of these finitevolume energies should contain information about the interactions, and thus information about scattering 13

The relation between the finite-volume spectrum and scattering amplitudes was worked out by Martin Lüscher, for the case of a single two-particle channel with zero total momentum [127, 128]. In this seminal work, Lüscher derived a relation that allows one to extract the two-pion elastic scattering phase-shift, $\delta_{J}$, and thus also the S-matrix, $S_{J}=\exp \left[2 i \delta_{J}\right]$, from the finite-volume spectrum.

\footnotetext{
${ }^{13}$ Consider also that the spectrum of QCD in finite-volume as well as the scattering properties of its low lying states are nonperturbative questions. Thus, although neither can be predicted analytically from the quark-field Lagrangian, it is possible that an analytic relation between them exists.
} 
The derivation proceeds by first providing a relation between low-energy QCD and nonrelativistic quantum mechanics. Specifically one can construct an energy-dependent potential $U_{E}$, such that the Schrödingier equation with $U_{E}$ gives a wave function with an asymptotic form dictated by $\delta_{J}$. Lüscher then provided a thorough analysis of the quantum mechanical system in finite-volume, to determine how the asymptotic wave-function may be extracted from the spectrum. The resulting expression gives the physical observables $\delta_{J}\left(E_{n}\right)$ from the discrete levels $E_{n}(L)$.

This work was further generalized to states with nonzero total momentum in the finitevolume frame [129, 130, 131]. Ref. [130] also provided an alternative, purely field theoretic derivation of Lüscher's quantization condition. To describe this generalization we introduce $\mathbf{P}$ as the total momentum of the system. We further introduce notation for the center of mass $(\mathrm{CM})$ frame spectrum $E_{1}^{*}(L), E_{2}^{*}(L), \cdots$. This is related to the moving frame spectrum via

$$
E^{*}=\sqrt{E^{2}-\mathbf{P}^{2}}
$$

The kinematic restriction mentioned above is now re-expressed as $0<E^{*}<4 m_{\pi}$.

In this thesis we generalize the result further to describe systems with any number of strongly coupled two-scalar channels. The particles may be identical or non-identical and degenerate or non-degenerate and may have nonzero total momentum. For example, our formalism accommodates the coupled $\pi \pi$ and $K \bar{K}$ system if coupling to four-(and higher)particle states is neglected.

Our derivation, presented in Chapter 2 below, follows the field-theoretic approach of Ref. [130]. Specifically, we diagrammatically evaluate a finite-volume two-to-two correlator to all orders in perturbation theory. As a result of the finite-volume condition, all momenta must satisfy $\mathbf{p} \in(2 \pi / L) \mathbb{Z}^{3}$. In particular, the loop momenta appearing in Feynman diagrams are summed over this discrete space, rather than integrated as in standard infinite-volume calculations. Since we are after infinite-volume quantities, our goal is to quantitatively relate the finite-volume sums with infinite-volume integrals. Following [130], we show in Chapter 2 that, if one neglects terms exponentially suppressed in $m_{\pi} L$, then it is possible to give a compact relation between finite-volume and infinite-volume diagrams. 
It is further possible to sum all diagrams to reach a simple expression for the finite-volume correlator. The poles in this correlator determine the finite-volume spectrum and so the analysis described gives a quantization condition for the energies of the compactified theory. Crucially, this quantization condition depends only on on-shell scattering amplitudes as well as known kinematic functions.

Preceding this work were a number of papers studying the generalization of the Lüscher quantization condition to multiple two-body channels [132, 133, 134, 135] and assessing its utility. The work of Ref. [132] uses non-relativistic quantum mechanics, while Ref. [133] is based on a non-relativistic effective field theory. References [134] and [135] are based on relativistic field theory, and give an explicit result [Eq. (3.5) of Ref. [134]] for the case of two s-wave channels in which the total momentum vanishes and in which the contributions from higher partial waves are assumed negligible. Our result is the first to consider nonzero total momentum and to accommodate all partial waves.

Going beyond the strong interaction, Lüscher's quantization condition can also be used to extract weak matrix elements from LQCD. In particular, one can derive a relation between the matrix element that is available on the lattice, connecting a single-particle state to a finite-volume two particle state, and the infinite volume matrix element which determines the decay rate. This result was provided by Lellouch and Lüscher in Ref. [70] (and generalized to a moving frame in Refs. [130, 131]). They showed that the relation between finite- and infinite-volume matrix elements of $\mathcal{H}_{W}$ emerges if one considers how Lüscher's result is modified when one perturbatively includes this weak hamiltonian as a shift to the QCD hamiltonian.

With the coupled channel quantization condition of Chapter 2 in hand, we show in Chapter 3 how to generalize the Lellouch-Lüscher result to extract weak decays into multiple strongly-coupled two-particle channels. In this case, each two-particle finite-volume state is associated with all of the infinite-volume two-particle asymptotic states. One thus finds that the finite-volume matrix element is equal to a linear combination of the infinite-volume matrix elements of interest.

An important limitation of the Lellouch-Lüscher approach is that it only provides matrix elements for scalar operators which do not inject energy or momentum into the system. 
Thus the derivation cannot accommodate processes with two-hadrons in the final state if these also include a momentum-carrying photon or lepton current. Using non-relativistic field theory, the authors of Ref. [136] provided an alternative approach for deriving matrix element relations like that of Lellouch and Lüscher. In Chapter 4 we present a modelindependent extension of this idea that is based in relativistic field theory. Our result is a relation of finite- and infinite-volume matrix elements of operators that insert arbitrary angular momentum as well as linear momentum and energy.

Finally, it is becoming increasingly important to also include three-hadron states in the extraction of scattering information from the finite-volume spectrum. This is a necessary step towards relating finite-volume matrix elements with infinite-volume matrix elements with three-particle states. This issue has been considered in Refs. [137, 138, 139] but a relativistic and model-independent relation is still unavailable. In Chapter 5 we derive a relativistic and model-independent relation between the three-particle finite-volume spectrum and a non-standard infinite-volume quantity. The derivation in similar in structure to that of Chapter 3. However the diagrams that appear are more complicated and the separation of finite-volume and infinite-volume expressions is more difficult. 
Chapter 2

\section{SCATTERING OF MULTIPLE TWO-PARTICLE CHANNELS ${ }^{1}$}

\subsection{Multiple-channel extension of quantization condition}

In this section we derive an extension to multiple two-body channels of the Lüscher quantization condition, which relates the infinite volume scattering amplitudes to finite volume energy levels. We assume throughout a cubic spatial volume with extent $L$ and periodic boundary conditions. The (Minkowski) time direction is taken to be infinite. The total momentum

$$
\vec{P}=\frac{2 \pi \vec{n}_{P}}{L} \quad\left(\vec{n}_{P} \in \mathbb{Z}^{3}\right)
$$

is fixed but arbitrary, i.e. the quantization condition we derive holds for a "moving frame" as well as a stationary frame. We first consider the case of only two open channels, describing the extension to an arbitrary number of channels at the end of this section.

We take each channel to contain two massive, spinless particles. The particles of channel one are labeled pions and are taken to be identical with mass $m_{1}=M_{\pi}$. The particles of channel two, called kaons, are taken non-identical, though still degenerate, with mass $m_{2}=M_{K}$. What we have in mind is that the first channel corresponds to the $I=0 \pi \pi$ state, and the second to the $I=0 K \bar{K}$ state. Including both identical and non-identical pairs allows us to display the factors of $1 / 2$ that appear in the former case. We consider degenerate particles to simplify the presentation, but describe the generalization to nondegenerate masses at the end of this section.

For concreteness, and to match the physical ordering, we take the pion to be lighter than the kaon. For our results to hold, we must assume that the thresholds for three or more particles lie above the two kaon threshold. If we assume a G-parity like symmetry, so that

\footnotetext{
${ }^{1}$ This chapter and the following chapter are taken from Ref. [140].
} 
only even numbers of pions can couple to a two-pion state, then the ordering we need is

$$
2 M_{\pi}<2 M_{K}<E^{*}<4 M_{\pi}
$$

where $E^{*}$ is the center of mass $(\mathrm{CM})$ energy. The only possible scattering events are then

$$
\begin{aligned}
& 1 \rightarrow 1: \quad \pi \pi \rightarrow \pi \\
& 1 \rightarrow 2: \quad \pi \pi \rightarrow K \bar{K} \\
& 2 \rightarrow 1: \quad K \bar{K} \rightarrow \pi \pi \\
& 2 \rightarrow 2: \quad K \bar{K} \rightarrow K \bar{K} .
\end{aligned}
$$

If $E^{*}$ drops below $2 M_{K}$, only the $\pi \pi$ channel is open and the problem reduces to that discussed by Lüscher [141, 127, 128, 142].

The inequality $2 M_{K}<4 M_{\pi}$ does not, of course, hold for physical pions and kaons - the four and six pion thresholds occur below that for two kaons. Nevertheless, the coupling to these higher multiplicity channels is weak at low energies, and our results should still hold approximately as long as we are not too far above the two kaon threshold. Indeed, it may be that, in the $I=0$ case, the $\eta \eta$ channel becomes important before that with four or more pions. If so, our formalism would still apply, generalized to three channels as described below. The approximation of ignoring channels with more than two particles will become increasingly poor as the energy increases, and will likely give only a rough guide by the $D$ mass. A qualitative indication of this (ignoring differences in phase space) is that the $f_{0}(1500)$ has a $50 \%$ branching fraction to $4 \pi$, while the branches to $\pi \pi, K \bar{K}$ and $\eta \eta$ are $\sim 35 \%, 9 \%$ and $5 \%$, respectively [69].

The two channel quantization condition is obtained by a straightforward generalization of the single-channel approach of Ref. [130]. To make this note somewhat independent of that reference, we reiterate some of the pertinent details. We begin by introducing a two body interpolating field $\sigma(x)$ (not necessarily local) which couples to both channels. Following Ref. [130] we then define

$$
C_{L}(P)=\int_{L ; x} e^{i\left(-\vec{P} \cdot \vec{x}+E x^{0}\right)}\left\langle 0\left|\sigma(x) \sigma^{\dagger}(0)\right| 0\right\rangle
$$


where $P=(E, \vec{P})$ is the total four momentum of the two particle system (in the frame where the finite volume condition is applied), and

$$
\int_{L ; x}=\int_{L} d^{4} x
$$

is the spacetime integral over finite volume. The relation to the CM energy used above is

$$
E^{*}=\sqrt{E^{2}-\vec{P}^{2}}
$$

The poles of $C_{L}$ give the energy spectrum of the finite volume theory, and thus the condition that $C_{L}$ diverge is precisely the quantization condition we are after.

To proceed to a more useful form of the condition, we follow Ref. [130] and write $C_{L}$ in terms of the Bethe-Salpeter kernel, as illustrated in Fig. 2.1(a):

$$
\begin{aligned}
C_{L}(P)=\int_{L ; q} \sigma_{j, q}\left[z^{2} \Delta^{2}\right]_{j k, q} & \sigma_{k, q}^{\dagger} \\
& \quad+\int_{L ; q, q^{\prime}} \sigma_{j, q}\left[z^{2} \Delta^{2}\right]_{j k, q} i K_{k l ; q, q^{\prime}}\left[z^{2} \Delta^{2}\right]_{l m, q^{\prime}} \sigma_{m, q^{\prime}}^{\dagger}+\cdots
\end{aligned}
$$

The notation here is as follows. Indices $j, k, l$ and $m$ refer to the channel, and take the values 1 or 2 . The two particle intermediate states are summed/integrated as is appropriate to finite volume

$$
\int_{L, q}=\frac{1}{L^{3}} \sum_{\vec{q}} \int \frac{d q^{0}}{2 \pi} .
$$

The summand/integrand includes the product of two fully dressed propagators

$$
\left[z^{2} \Delta^{2}\right]_{i j, q}=\delta_{i j} \eta_{i}\left[z_{i}(q) \Delta_{i}(q)\right]\left[z_{i}(P-q) \Delta_{i}(P-q)\right]
$$

where

$$
\begin{aligned}
z_{j}(q) \Delta_{j}(q) & =\int d^{4} x e^{i q x}\left\langle\phi_{j}(x) \phi_{j}(0)\right\rangle \\
\Delta_{j}(q) & =\frac{i}{q^{2}-m_{j}^{2}+i \epsilon} .
\end{aligned}
$$

Here $\phi_{1}$ and $\phi_{2}$ are interpolating fields for pions and kaons, respectively, chosen such that $z_{j}=1$ on shell. $\eta_{1}=1 / 2$ and $\eta_{2}=1$ account for the symmetry factors of the diagrams. $K$ is related to the Bethe-Salpeter kernel

$$
i K_{i j ; q, q^{\prime}}=i B S_{i j}\left(q, P-q,-q^{\prime},-P+q^{\prime}\right)
$$


a)

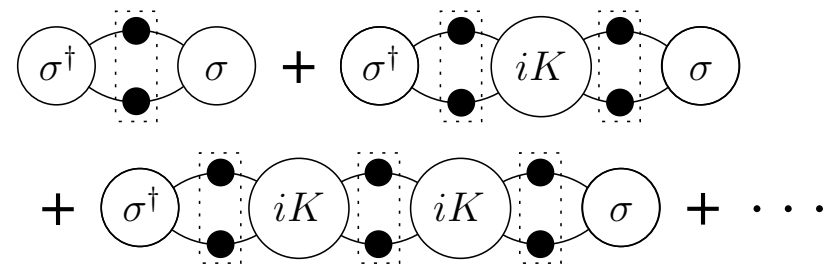

b)

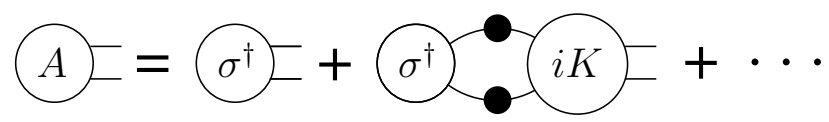

c)

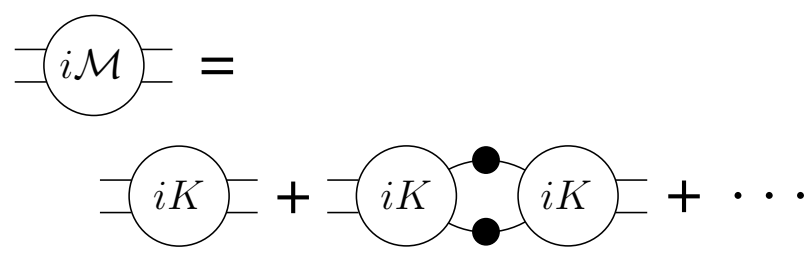

d)

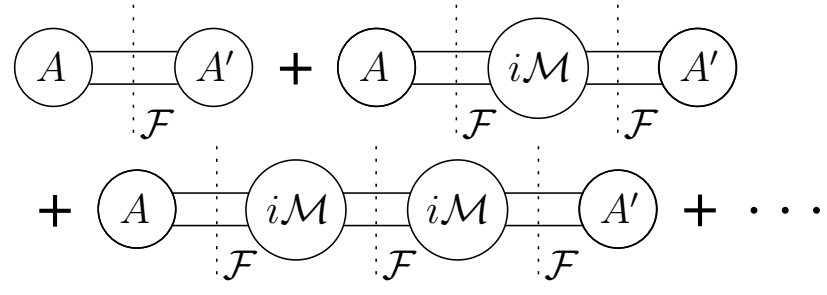

Figure 2.1: (a) The initial series of ladder diagrams which builds up $C_{L}$ [see Eq. 2.7)]. The Bethe-Salpeter kernels $i K$ are connected by fully dressed propagators. The dashed rectangle indicates finite volume momentum sum/integrals. (b) and (c) The series which build up the matrix element $A$ and the scattering amplitude $i \mathcal{M}$. Note that these series contain only the momentum integrals appropriate to infinite volume. (d) The resulting series for the subtracted correlator [see Eqs. 22.17) and 2.26)]. Each dashed vertical line indicates an insertion of $\mathcal{F}$, which carries the entire volume dependence (neglecting exponentially suppressed dependence). 
with $B S_{i j}$ the sum of all amputated $j \rightarrow i$ scattering diagrams which are two-particleirreducible in the $s$-channel (with particles of either type). Finally, $\sigma_{j, q}$ and $\sigma_{j, q^{\prime}}^{\dagger}$ describe the coupling of the operators $\sigma$ and $\sigma^{\dagger}$ to the two-particle channel $j$. Their detailed form is not relevant; all we need to know is that they are regular functions of $q$.

We emphasize two important features of Eq. 2.7). First, it does not rely on any choice of interactions between the pions and kaons, such as those predicted by chiral perturbation theory. All the quantities that enter can be written in terms of non-perturbatively defined correlation functions. Second, the kernel $i K$ and the propagator dressing function $z$ have only exponentially suppressed dependence on the volume [127]. Thus, if $L$ is large enough that such dependence is negligible (as we assume hereafter), we can take $i K$ and $z$ to have their infinite-volume forms.

The dominant power-law volume dependence enters through the momentum sums in the two-particle loops. To extract this dependence, we use the identity derived in Ref. [130], which relates these sums for a moving frame to infinite-volume momentum integrals plus a residue. Before stating the identity we recall the relevant notation. For any four vector $k^{\mu}=\left(k^{0}, \vec{k}\right)$ in the moving frame, $k^{\mu *}=\left(k^{0 *}, \vec{k}^{*}\right)$ is the result of a boost to the CM frame. In particular, the total four-momentum $(E, \vec{P})$ boosts to $\left(E^{*}, \overrightarrow{0}\right)$ in the CM frame. We also need the quantities

$$
q_{j}^{*}=\sqrt{\left(E^{*}\right)^{2} / 4-m_{j}^{2}},
$$

which are the momenta of a pion $(j=1)$ or kaon $(j=2)$ in the CM frame. The identity then reads (no sum on $i$ here):

$$
\begin{aligned}
& \int_{L ; k} f(k) \eta_{i} \Delta_{i}(k) \Delta_{i}(P-k) g(k)= \\
& \quad \int_{\infty ; k} f(k) \eta_{i} \Delta_{i}(k) \Delta_{i}(P-k) g(k)+\int d \Omega_{q^{*}} d \Omega_{q^{*^{\prime}}} f_{i}^{*}\left(\hat{q}^{*}\right) \mathcal{F}_{i i}\left(\hat{q}^{*}, \hat{q}^{*}\right) g_{i}^{*}\left(\hat{q}^{\prime}\right),
\end{aligned}
$$

with

$$
\int_{\infty ; k}=\int \frac{d^{4} k}{(2 \pi)^{4}} .
$$

We introduce two functions $f(k)$ and $g(k)$ to correspond to the momentum dependence entering from the left and right of the loop integrals, as well as that from the dressing functions [see Fig. 2.1(a)]. The functions $f$ and $g$ must have ultraviolet behavior that renders 
the integral/sum convergent. In addition, the branch cuts they contain, corresponding to four or more intermediate particles, must be such that, after the $k^{0}$ contour integration, they introduce no singularities for real $\vec{k}$. This condition holds when $0<E^{*}<4 M_{\pi}$. The last line of (2.14) depends on the values of the functions $f$ and $g$ when the two particles are on-shell, and thus only on the direction of the CM momentum, $\hat{q}^{*}$. Specifically, if $q_{i}^{\mu}$ is the moving frame momentum that boosts to the on-shell momentum $\left(E^{*} / 2, \vec{q}_{i}{ }^{*}\right)$, then

$$
f_{i}^{*}\left(\hat{q}^{*}\right)=f\left(q_{i}\right), \quad g_{i}^{*}\left(\hat{q}^{*}\right)=g\left(q_{i}\right)
$$

Finally the quantity $\mathcal{F}$, which depends on $q^{*}, L$ and the particle mass, contains the powerlaw finite-volume dependence of the loop sum/integral $\left.\right|^{2}$ Its form is given below in Eqs. (2.23)(2.25). Note that it is diagonal in channel space, i.e. it cannot change pions into kaons. It can, however, insert angular momentum, due to the breaking of rotation symmetry by the cubic box.

The key point of the identity is that the difference between finite and infinite volume integrals depends on on-shell values of the integrand, allowing the finite-volume dependence to be expressed in terms of physical quantities. Applying the identity to each loop integral in Fig. 2.1(a), one then rearranges the series by grouping terms with the same number of insertions of $\mathcal{F}$. The volume-independent term with no $\mathcal{F}$ insertions is of no interest, since it does not lead to poles. Thus we drop it and consider the difference

$$
C_{\text {sub }}(P) \equiv C_{L}(P)-C_{\infty}(P)
$$

In the remaining diagrams with $\mathcal{F}$ insertions, all terms to the left of the first $\mathcal{F}$ and to the right of the last are grouped and summed into new endcaps which we label $A_{j}$ and $A_{j}^{\prime}$ [see Fig. 2.1(b)]. These quantities equal certain matrix elements of the interpolating field $\sigma$ [130]

$$
\begin{aligned}
& A_{j}\left(\hat{k}^{*}\right) \equiv\left\langle\vec{k}^{*},-\vec{k}^{*} ; j ; \text { out }\left|\sigma^{\dagger}(0)\right| 0\right\rangle_{\left|\vec{k}^{*}\right|=q_{j}^{*}} \\
& A_{j}^{\prime}\left(\hat{k}^{*}\right) \equiv\left\langle 0|\sigma(0)| \vec{k}^{*},-\vec{k}^{*} ; j ; \text { in }\right\rangle_{\left|\vec{k}^{*}\right|=q_{j}^{*}} .
\end{aligned}
$$

\footnotetext{
${ }^{2}$ The result 2.14) is equivalent to Eqs. (41-42) of Ref. 130, although we have done some further manipulations to the last line of 2.14 to bring it into a matrix form. Also, we have included a factor of $\eta_{i}$ in $\mathcal{F}$, rather than keeping it explicitly as in Ref. [130].
} 
In contrast to [130] we include no wavefunction renormalization factors, because our single particle interpolating fields satisfy on-shell renormalization conditions. Having summed up the ends the next step is to do the same for the series which appears between adjacent $\mathcal{F}$ insertions [Fig. 2.1(c)]. As indicated in the figure, this series generates the infinite volume scattering amplitude $i \mathcal{M}_{i j}$. We thus deduce an alternative series for $C_{s u b}$ built from $A, A^{\prime}$ and $i \mathcal{M}$ s, all connected by $\mathcal{F}_{\mathrm{S}}[\mathrm{Fig}$. 2.1(d)].

We stress that the analysis just performed is a straightforward generalization of the single channel analysis of Ref. [130]. All that has changed is that $\mathcal{F}$ and $\mathcal{M}$ are now $2 \times 2$ matrices in channel space, and $A$ and $A^{\prime}$ vectors.

To proceed, we decompose $A, A^{\prime}, \mathcal{M}$ and $\mathcal{F}$ in spherical harmonics, defining coefficients via

$$
\begin{aligned}
A_{j}\left(\hat{k}^{*}\right) & \equiv \sqrt{4 \pi} A_{j ; \ell, m} Y_{\ell, m}\left(\hat{k}^{*}\right) \\
A_{j}^{\prime}\left(\hat{k}^{*}\right) & \equiv \sqrt{4 \pi} A_{j ; \ell, m}^{\prime} Y_{\ell, m}^{*}\left(\hat{k}^{*}\right) \\
\mathcal{M}_{i j}\left(\hat{k}^{*}, \hat{k}^{\prime}\right) & \equiv 4 \pi \mathcal{M}_{i j ; \ell_{1}, m_{1} ; \ell_{2}, m_{2}} Y_{\ell_{1}, m_{1}}\left(\hat{k}^{*}\right) Y_{\ell_{2}, m_{2}}^{*}\left(\hat{k}^{*}\right) \\
\mathcal{F}_{i j}\left(\hat{k}^{*}, \hat{k}^{\prime}\right) & \equiv-\frac{1}{4 \pi} F_{i j ; \ell_{1}, m_{1} ; \ell_{2}, m_{2}} Y_{\ell_{1}, m_{1}}\left(\hat{k}^{*}\right) Y_{\ell_{2}, m_{2}}^{*}\left(\hat{k}^{*}\right),
\end{aligned}
$$

where a sum over all $\ell$ 's and $m$ 's is implicit. The factors of $4 \pi$ are present so that we match the conventions of Ref. [130]. They imply, for example, that for a purely s-wave amplitude, $\mathcal{M}$ is the same in the two bases (for the $4 \pi$ cancels with the two spherical harmonics). The kinematical factor $F$ is given in Ref. [130] (aside from the above-noted factor of $\eta_{i}$ ) and takes the form 3

$$
\begin{aligned}
& F_{i j ; \ell_{1}, m_{1} ; \ell_{2}, m_{2}} \equiv \delta_{i j} F_{i ; \ell_{1}, m_{1} ; \ell_{2}, m_{2}} \\
&=\delta_{i j} \eta_{i}\left[\frac{\operatorname{Re} q_{i}^{*}}{8 \pi E^{*}} \delta_{\ell_{1} \ell_{2}} \delta_{m_{1} m_{2}}-\frac{i}{2 E^{*}} \sum_{\ell, m} \frac{\sqrt{4 \pi}}{q_{i}^{* \ell}} c_{\ell m}^{P}\left(q_{i}^{* 2}\right) \int d \Omega Y_{\ell_{1}, m_{1}}^{*} Y_{\ell, m}^{*} Y_{\ell_{2}, m_{2}}\right]
\end{aligned}
$$

${ }^{3}$ An additional difference from Ref. [130 is the appearance of $\operatorname{Re} q_{i}^{*}$ rather than $q^{*}$. This is discussed in the next section. 
Here the volume-dependence enters through the sum: $4^{4}$

$$
c_{\ell m}^{P}\left(q^{* 2}\right)=\frac{1}{L^{3}} \sum_{\vec{k}} \frac{\omega_{k}^{*}}{\omega_{k}} \frac{e^{\alpha\left(q^{* 2}-k^{* 2}\right)}}{q^{* 2}-k^{* 2}} k^{* \ell} \sqrt{4 \pi} Y_{\ell, m}\left(\hat{k}^{*}\right)-\delta_{\ell 0} \mathcal{P} \int \frac{d^{3} k^{*}}{(2 \pi)^{3}} \frac{e^{\alpha\left(q^{* 2}-k^{* 2}\right)}}{q^{* 2}-k^{* 2}},
$$

with $\omega_{k}=\sqrt{\vec{k}^{2}+m_{i}^{2}}$ being the energy of a particle with momentum $\vec{k}$, and $\omega_{k}^{*}$ the energy after boosting to the CM frame. The properties of these sums are discussed in Ref. [130].

We are now in a position to write down the final result. The series indicated in Fig. 2.1(d) gives

$$
\begin{aligned}
C_{\text {sub }}(P) & =-\sum_{n=0}^{\infty} A^{\prime} F[-i \mathcal{M} F]^{n} A, \\
& =-A^{\prime} \frac{1}{F^{-1}+i \mathcal{M}} A .
\end{aligned}
$$

Here all indices are left implicit and may be restored in the obvious way. For example,

$$
A^{\prime} F \mathcal{M F A}=A_{i ; \ell_{1}, m_{1}}^{\prime} F_{i j ; \ell_{1}, m_{1} ; \ell_{2}, m_{2}} \mathcal{M}_{j k ; \ell_{2}, m_{2} ; \ell_{3}, m_{3}} F_{k l ; \ell_{3}, m_{3} ; \ell_{4}, m_{4}} A_{l, \ell_{4}, m_{4}} .
$$

As $C_{\infty}$ has no poles in the region of $E^{*}$ that we consider (below $4 M_{\pi}$ ), the poles in $C_{L}$ must match the poles in $C_{\text {sub }}$. The desired quantization condition is then just that the matrix between $A^{\prime}$ and $A$ have a divergent eigenvalue. This may be written as

$$
\operatorname{det}\left(F^{-1}+i \mathcal{M}\right)=0
$$

where we recall that the matrices now act in the product of the two-dimensional channel space and the infinite-dimensional angular-momentum space. More precisely, $F$ is diagonal in channel space but has off-diagonal elements between different angular momentum sectors (as allowed by the symmetries of the cubic box and the momentum $\vec{P}$ ), while $\mathcal{M}$ is diagonal in angular momentum but off-diagonal in channel space.

Equation 2.29 is the main result of this section. It has exactly the same form as that for the single channel given in Ref. [130] (aside from the change of notation in which symmetry

\footnotetext{
${ }^{4}$ We are slightly abusing the notation here for the sake of clarity. $c_{\ell m}^{P}$ depends not only on $q^{* 2}$ but also on $m_{i}$, but we keep the latter dependence implicit. The dependence is made explicit at the end of this section.
} 
factors are contained in $F$ rather than kept explicit). The generalization to more than two two-particle channels is now immediate. As long as $E^{*}$ is kept below the four particle threshold of the lightest particle the arguments above go through in the same manner. One need only extend the values of the channel indices, taking care to include the appropriate symmetry factor $\eta_{j}$ for each channel. The final result then has exactly the form of Eq. (2.29).

To make the formal expression (2.29) useful in practice one assumes that there is some $\ell_{\max }$, above which the partial wave amplitudes are negligible

$$
\mathcal{M}_{i j}^{\ell>\ell_{\max }}=0 .
$$

One can then show that, although $F$ couples $\ell \leq \ell_{\max }$ to $\ell>\ell_{\max }$, the projection contained in $\mathcal{M}$ is sufficient to collapse the required determinant to that in the $\ell \leq \ell_{\text {max }}$ subspace. The argument for this result is given for one channel in Ref. [130] and generalizes trivially to the multiple channel case. Thus one finds that Eq. 2.29) still holds, but with $\mathcal{M}$ and $F$ now understood to be finite dimensional matrices both in channel space and in the partial wave basis, with $\ell$ running up to $\ell_{\max }$.

To conclude this section we comment briefly on two generalizations of the result. We first consider the case when not just a single $\sigma$ but rather a set of operators $\left\{\sigma^{a}\right\}$ is of interest. This is likely to be the case in practice since multiple operators may be needed to find combinations with good overlaps with the finite-volume eigenstates. If there are $n$ such operators, then $C_{L}$ generalizes to an $n \times n$ matrix:

$$
C_{L}^{a b}(P)=\int_{L ; x} e^{i\left(-\vec{P} \cdot \vec{x}+E x^{0}\right)}\left\langle 0\left|\sigma^{a}(x) \sigma^{\dagger b}(0)\right| 0\right\rangle .
$$

The generalization of Eq. (2.27) is effected by replacing $A^{\prime}$ with an $n \times 2$ matrix

$$
\left(\begin{array}{ll}
A_{1}^{\prime} & A_{2}^{\prime}
\end{array}\right) \longrightarrow\left(\begin{array}{cc}
A_{1}^{\prime a=1} & A_{2}^{\prime a=1} \\
A_{1}^{\prime a=2} & A_{2}^{\prime a=2} \\
\vdots & \vdots
\end{array}\right)
$$

and $A$ with a $2 \times n$ matrix

$$
\left(\begin{array}{c}
A_{1} \\
A_{2}
\end{array}\right) \longrightarrow\left(\begin{array}{ccc}
A_{1}^{b=1} & A_{1}^{b=2} & \ldots \\
A_{2}^{b=1} & A_{2}^{b=2} & \ldots
\end{array}\right)
$$


The key point, however, is that the matrix between $A^{\prime}$ and $A$ is unchanged, so that the quantization condition 2.29 is unaffected. This is as expected, since the operators used to couple to states cannot affect the eigenstates themselves.

The second generalization is to the case of non-degenerate particles. The expressions given above remain valid as long as one makes three changes. First, the symmetry factors $\eta_{i}$ become unity for all non-degenerate channels. Second, $q_{i}^{*}$ in Eqs. 2.24) is replaced by the solution of

$$
E^{*}=\sqrt{q_{i}^{* 2}+M_{i a}^{2}}+\sqrt{q_{i}^{* 2}+M_{i b}^{2}},
$$

which is the CM momentum when the channel contains particles of masses $M_{i a}$ and $M_{i b}$. Third, when evaluating $c_{\ell m}^{P}$ using Eq. 2.25, one should use one of the masses $M_{i a}$ or $M_{i b}$ when determining $\omega_{k}, \omega_{k}^{*}$ and $\vec{k}^{*}$. One can show that both choices lead to the same result.

The third change emphasizes that the kinematic functions $c_{\ell m}^{P}$ depend not only on $q_{i}^{*}$ but also on the particle masses. This can be made explicit by rewriting them in terms of a generalization of the zeta-function introduced in Ref. [129]. The result is [143, 144, 145, 146]

$$
\begin{aligned}
c_{\ell m}^{P}\left(q^{* 2}\right) & =-\frac{\sqrt{4 \pi}}{\gamma L^{3}}\left(\frac{2 \pi}{L}\right)^{\ell-2} \mathcal{Z}_{\ell m}^{P}\left[1 ;\left(q^{*} L / 2 \pi\right)^{2}\right], \\
\mathcal{Z}_{\ell m}^{P}\left[s ; x^{2}\right] & =\sum_{\vec{n}} \frac{r^{\ell} Y_{\ell m}(\hat{r})}{\left(r^{2}-x^{2}\right)^{s}}
\end{aligned}
$$

where $\gamma=E / E^{*}, \vec{n}$ runs over integer vectors, and $\vec{r}$ is obtained from $\vec{n}$ by $r_{\|}=\gamma^{-1}\left[n_{\|}-c \vec{n}_{P}\right]$ and $r_{\perp}=n_{\perp}$, where parallel and perpendicular are relative to $\vec{P}$, and $2 c=\left(1+\left(M_{1 a}^{2}-\right.\right.$ $\left.\left.M_{1 b}^{2}\right) / E^{* 2}\right)$. The sum in $\mathcal{Z}_{\ell m}$ can be regulated by taking $s>(3+\ell) / 2$ and then analytically continuing to $s=1$. This shows that mass dependence enters through the difference 5 $M_{i a}^{2}-M_{i b}^{2}$. One can derive 2.35 by generalizing the method used for the degenerate case in Ref. [130.

\footnotetext{
${ }^{5}$ The apparent lack of symmetry under the interchange $M_{i a} \leftrightarrow M_{i b}$ can be understood as follows. One can show that $\mathcal{Z}_{\ell m}^{P} \rightarrow(-)^{\ell} \mathcal{Z}_{\ell m}^{P}$ under this interchange (so that for degenerate masses the zeta-functions for odd $\ell$ vanish [129]). This sign flip for odd $\ell$ must hold also for the $c_{\ell m}^{P}$, and it does because the interchange of masses leads to $\vec{k}^{*} \rightarrow-\vec{k}^{*}$ at the pole. The sign flip is canceled in the expression for $\mathcal{F}$, Eq. 2.23, since the product $Y_{\ell_{1}, m_{1}}\left(\vec{k}^{*}\right) Y_{\ell_{2}, m_{2}}^{*}\left(\vec{k}^{*}\right)$ also changes sign. This is because, when $\ell$ is odd, the integral over $d \Omega$ in the definition of $F$, Eq. 2.24, enforces that $\ell_{1}+\ell_{2}$ is odd. The overall effect is that the quantization condition is symmetric under mass interchange, as it must be.
} 


\subsection{Multiple-channel quantization condition for s-wave scattering}

For the remainder of this article we focus on the simplest case, $\ell_{\max }=0$, in which only s-wave scattering is significant. In this section we determine the explicit form for the finitevolume quantization condition when there are two channels. We also present compact forms for the condition when an arbitrary number of two particle channels are open.

With only s-wave scattering, the two channel quantization condition takes the form

$$
\left[\left(F_{1}^{s}\right)^{-1}+i \mathcal{M}_{11}^{s}\right]\left[\left(F_{2}^{s}\right)^{-1}+i \mathcal{M}_{22}^{s}\right]-\left[i \mathcal{M}_{12}^{s}\right]\left[i \mathcal{M}_{21}^{s}\right]=0
$$

where

$$
\begin{aligned}
F_{i}^{s} & =\eta_{i}\left[\frac{\operatorname{Re} q_{i}^{*}}{8 \pi E^{*}}-\frac{i}{2 E^{*}} c_{i}^{P}\right] \\
c_{i}^{P} & \equiv c^{P}\left(q_{i}^{* 2}\right) \equiv c_{00}^{P}\left(q_{i}^{* 2}\right),
\end{aligned}
$$

and the superscript on $F$ and $\mathcal{M}$ is a reminder that only $\ell=0$ contributes.

To simplify Eq. (2.37), and in particular to re-express it as an equation between real quantities, it is useful to recall first the single-channel analysis. This has the additional benefit of showing how the two-channel result collapses to the known single-channel result in the appropriate kinematic regime, namely

$$
2 M_{\pi}<E^{*}<2 M_{K}
$$

In this regime $q_{2}^{*}$ becomes imaginary, and the second channel contributes negligibly because $c^{P}$ [Eq. 2.25)] becomes exponentially volume-suppressed and $\operatorname{Re} q^{*}$ in $F_{2}$ [Eq. (2.24] ] vanishes ${ }^{6}$ Sending $F_{2} \rightarrow 0$ we find that the quantization condition becomes

$$
\left[\mathcal{M}_{11}^{s}\right]^{-1}=\eta_{1}\left[-\frac{i q_{1}^{*}}{8 \pi E^{*}}-\frac{1}{2 E^{*}} c^{P}\left(q_{1}^{* 2}\right)\right]
$$

Note that the pion momentum $q_{1}^{*}$ is real for the energy region considered.

\footnotetext{
${ }^{6}$ The appearance of $\operatorname{Re} q^{*}$ rather than $q^{*}$ in $F_{i}$ can be understood by reviewing the derivation of $F$ in Ref. 130. The term enters as the difference between principal part and $i \epsilon$ prescriptions. When $q^{*}$ is imaginary there is no pole and different ways of regulating give the same result.
} 
Naively one might think that Eq. 2.41) gives two conditions, the separate vanishing of the real and imaginary parts. This is not the case, however, because the vanishing of the imaginary part is a volume-independent condition which is guaranteed to hold by the unitarity of the $S$-matrix. This can be seen by expressing $\mathcal{M}$ in terms of the real phase shift $\delta\left(q^{*}\right)$,

$$
\mathcal{M}_{11}^{s}=\frac{8 \pi E^{*}}{\eta_{1} q_{1}^{*}}\left[\frac{e^{2 i \delta\left(q_{1}^{*}\right)}-1}{2 i}\right]=\left[\frac{\eta_{1} q_{1}^{*}}{8 \pi E^{*}}\left[\cot \delta\left(q_{1}^{*}\right)-i\right]\right]^{-1} .
$$

Here $e^{2 i \delta}$ is the one dimensional unitary $S$-matrix in the partial wave basis. Given Eq. 2.42, it is manifest that the imaginary part of Eq. 2.41 holds automatically. The real part of (2.41) then gives the moving frame generalization of the Lüscher result in the familiar partial wave form [128, 130, 131 ]

$$
\tan \delta\left(q_{1}^{*}\right)=-\tan \phi^{P}\left(q_{1}^{*}\right)
$$

where

$$
\tan \phi^{P}\left(q^{*}\right)=\frac{q^{*}}{4 \pi}\left[c^{P}\left(q^{* 2}\right)\right]^{-1} .
$$

We now return to the CM energies for which both channels are open, $2 M_{K}<E^{*}<4 M_{\pi}$, and generalize Eq. 2.43). The first step is to recall the relationship between the scattering amplitude and the $S$-matrix. Unitarity implies that

$$
\mathcal{M}^{s}-\mathcal{M}^{s \dagger}=i \mathcal{M}^{s \dagger} P^{2} \mathcal{M}^{s}
$$

where $P^{2}$ is a diagonal matrix containing the phase-space factors, whose square root is

$$
P=\frac{1}{\sqrt{4 \pi E^{*}}}\left(\begin{array}{cc}
\sqrt{\eta_{1} q_{1}^{*}} & 0 \\
0 & \sqrt{\eta_{2} q_{2}^{*}}
\end{array}\right) .
$$

We note that, when expressed in terms of $q^{*}$, the form of $P$ is still valid if the two particles in the channel are non-degenerate. We also note that the form 2.45 holds for an arbitrary number of two-particle, s-wave channels, with $P$ generalized in the obvious way.

The solution to the unitarity relation is

$$
i \mathcal{M}^{s}=P^{-1}\left(S^{s}-1\right) P^{-1}
$$


where $S^{s}$ is a dimensionless, unitary $2 \times 2$ matrix. To proceed, we need to parametrize $S^{s}$. First we note that $S^{s}$ can be taken to be symmetric. This is because of the T-invariance of the strong interactions, together with the fact that angular momentum eigenstates have definite T-parity (in our case, positive). Thus in the $2 \times 2$ case, $S^{s}$ is determined by three real parameters. We use the "eigenphase convention" of Blatt and Biedenharn [147],

$$
S^{s}=\left(\begin{array}{cc}
\mathrm{c}_{\epsilon} & -\mathrm{s}_{\epsilon} \\
\mathrm{s}_{\epsilon} & \mathrm{c}_{\epsilon}
\end{array}\right)\left(\begin{array}{cc}
e^{2 i \delta_{\alpha}} & 0 \\
0 & e^{2 i \delta_{\beta}}
\end{array}\right)\left(\begin{array}{cc}
\mathrm{c}_{\epsilon} & \mathrm{s}_{\epsilon} \\
-\mathrm{s}_{\epsilon} & \mathrm{c}_{\epsilon}
\end{array}\right),
$$

where the notation $\mathrm{s}_{x}=\sin x$ and $\mathrm{c}_{x}=\cos x$ will be used throughout. The three real parameters $\delta_{\alpha}, \delta_{\beta}$, and $\epsilon$ generalize the single $\delta$ which appears in the one channel case. The parameter $\epsilon$ quantifies the mixing between the mass eigenstates of channels one and two (the pions and kaons) and the $S$-matrix eigenstates. The phases $\delta_{\alpha}$ and $\delta_{\beta}$ are, for arbitrary $\epsilon$, associated with both channels. Of course, in the limit $\epsilon \rightarrow 0$ they reduce, respectively, to the phase shifts of pion and kaon elastic scattering.

Substituting the form of $S^{s}$ into Eq. 2.47) and then placing this in Eq. 2.37) and simplifying, we deduce 7

$$
\begin{aligned}
& {\left[\tan \delta_{\alpha}+\tan \phi^{P}\left(q_{1}^{*}\right)\right]\left[\tan \delta_{\beta}+\tan \phi^{P}\left(q_{2}^{*}\right)\right]} \\
& \quad+\sin ^{2} \epsilon\left[\tan \delta_{\alpha}-\tan \delta_{\beta}\right]\left[\tan \phi^{P}\left(q_{1}^{*}\right)-\tan \phi^{P}\left(q_{2}^{*}\right)\right]=0 .
\end{aligned}
$$

This is the main result of this section. One can use it in one of two ways: to predict the spectrum given knowledge of the scattering amplitude from experiment or a model, or to determine the $S$-matrix parameters from a lattice calculation of the spectrum. In the former case, we note that all quantities appearing in 2.49 , i.e. $\delta_{\alpha}, \delta_{\beta}, \epsilon, q_{i}^{*}$ and $\phi^{P}$, are functions

\footnotetext{
${ }^{7}$ We emphasize that the physical content of Eq. 2.49, namely that there is a relation between scattering amplitudes and energy levels, does not depend on the parametrization chosen for the matrix $S^{s}$. This is clear either from Eq. 2.37) or from Eq. 2.58 below. An advantage of our choice of parametrization is that it shows that Eq. 2.37) only implies one real condition (rather than two), an observation which must hold for any parameterization. We also note that the freedom to independently change the phases of $\pi \pi$ and $K \bar{K}$ states, which leads to $S^{s} \rightarrow U^{\dagger} S^{s} U$, with $U$ a diagonal unitary matrix, does not change the quantization condition, as can be seen most easily from Eq. 2.58 below.
} 
of $E^{*}$. One can thus search, at given spatial extent $L$ and total momentum $\vec{P}$, for values of $E^{*}$ which satisfy Eq. (2.49). If the condition holds for a particular $E_{k}^{*}$, then

$$
E_{k}\left(L ; \vec{n}_{P}\right)=\sqrt{E_{k}^{* 2}+\vec{P}^{2}}
$$

is in the spectrum of the finite-volume moving-frame Hamiltonian. Here we choose to write $E_{k}$ as a function of $\vec{n}_{P}$ rather than $\vec{P}$, since, in practice, it is the former quantity which is held fixed as one varies $L$.

The second use of 2.49 is the most relevant for the discussion in subsequent sections. For a given choice of $E^{*}$, one finds, through a lattice calculation, three pairs $\left\{L, \vec{n}_{P}\right\}$ for which there is a spectral line $E_{k}$ such that $E_{k}^{*}$ [defined in Eq. [2.50]] is equal to $E^{*}$. One could use a fixed $\vec{n}_{P}$ and consider multiple spectral lines (the simplest choice conceptually), or use three different choices of $\vec{n}_{P}$ (probably more practical since one would not need to determine so many excited levels). In either case, one ends up with three versions of Eq. (2.49), all containing the desired quantities $\delta_{\alpha}\left(E^{*}\right), \delta_{\beta}\left(E^{*}\right)$ and $\epsilon\left(E^{*}\right)$, but having different values of the $\phi^{P}\left(q_{j}^{*}\right)$. Solving these equations one determines, rather than the angles themselves, the quantities $\tan \delta_{\alpha}, \tan \delta_{\beta}$, and $\sin ^{2} \epsilon$ at $\mathrm{CM}$ energy $E^{*}$. For our discussion we therefore restrict

$$
\delta_{\alpha, \beta} \in[-\pi / 2, \pi / 2] \text { and } \epsilon \in[0, \pi / 2] .
$$

Having determined the restricted phases over a range of energies, one can afterward relax the constraints in order to build continuous curves as a function of energy. We direct the reader to Refs. [134, 135] for discussion of other methods for extracting the three scattering parameters.

We emphasize that Eq. (2.49) has a very intuitive form. If $\delta_{\alpha}=\delta_{\beta}$ or $m_{1}=m_{2}$ or $\epsilon=0$ then the second line vanishes and the result reduces to two copies of the one channel quantization condition [Eq. (2.43)]. To see that this makes sense, note that for identical phase shifts, the $\epsilon$ matrix commutes through the phase matrix and we recover two uncoupled channels. Similarly if the masses are degenerate then the eigenstates of the $S$-matrix will also be mass eigenstates leading to the decoupled form. Finally, the decoupling for $\epsilon=0$ is an obvious property of the parametrization [Eq. (2.48)]. 
An alternative solution to the unitarity relation 2.45 can be given in terms of the $K$-matrix used in Ref. [134]. Specifically, 2.45) is satisfied if

$$
\left(\mathcal{M}^{s}\right)^{-1}=M-i P^{2} / 2
$$

with $M$ any real symmetric $2 \times 2$ matrix. If we set

$$
M=\frac{1}{8 \pi E^{*}}\|\sqrt{\eta}\| K^{-1}\|\sqrt{\eta}\|,
$$

[where double bars denote a diagonal matrix, so that $\left.\|\eta\|=\operatorname{diag}\left(\sqrt{\eta_{1}}, \sqrt{\eta_{2}}\right)\right]$, and further set $\vec{P}=0$, then one can show that the two-channel quantization condition given above can be manipulated into the form given in Eq. (3.5) of Ref. [134] in terms of the real, symmetric matrix $K$.

We now generalize Eq. (2.49) to $N$ s-wave channels. As noted above, the form of the unitarity relation 2.45 holds for any $N$, and the same is true for its solution (2.47). In the latter, the $N$ channel $S$-matrix can be parametrized as 8

$$
S=R^{-1}\left\|e^{2 i \delta}\right\| R
$$

with $R$ an $S O(N)$ matrix, and

$$
\left\|e^{2 i \delta}\right\|=\operatorname{diag}\left[e^{2 i \delta_{\alpha}}, e^{2 i \delta_{\beta}}, \cdots\right]
$$

Together with Eqs. (2.54) and (2.55) one needs the $N \times N$ generalization of $F$, which has been discussed in the previous section. From these definitions one can straightforwardly work out the quantization condition for $N$ coupled channels.

We conclude this section by describing two additional ways of writing the quantization condition, both of which make the higher channel generalization especially clear. Observe first that, for any number of open channels,

$$
F^{-1}=P^{-1}\left\|1-e^{-2 i \phi}\right\| P^{-1}
$$

Combining this with 2.47), it follows that

$$
F^{-1}+i \mathcal{M}=P^{-1}\left[S-\left\|e^{-2 i \phi}\right\|\right] P^{-1}
$$

\footnotetext{
${ }^{8}$ The remainder of this paper is limited to the $s$-wave, so we drop the superscript ${ }^{s}$ hereafter.
} 
Since $P^{-1}$ has no singularities in the kinematic regime we consider, the quantization condition can be rewritten as

$$
\operatorname{det}\left[S-\left\|e^{-2 i \phi}\right\|\right]=0 \text {. }
$$

This form shows that the symmetry factors cancel from the quantization condition in general. Although Eq. (2.58) looks like it will lead to one complex and thus two real conditions, it turns out that it leads only to a single real condition. This follows from the identity

$$
\left\|1+i t_{\phi}\right\| \times\left[S-\left\|e^{-2 i \phi}\right\|\right] \times\left[R^{-1}\left\|1-i t_{\delta}\right\| R\right]=2 i\left[R^{-1}\left\|t_{\delta}\right\| R+\left\|t_{\phi}\right\|\right]
$$

where $t_{x}=\tan x$. It gives a manifestly real rewriting of the quantization condition,

$$
\operatorname{det}\left[R^{-1}\left\|t_{\delta}\right\| R+\left\|t_{\phi}\right\|\right]=0 .
$$

This form leads directly to the result (2.49) in the two channel case, and collapses to the single-channel result (2.43) for any channel that decouples from the rest. If any of the channels contain non-degenerate particles, this enters only through the values of the kinematic functions $t_{\phi}$, as discussed in the previous section. 
Chapter 3

\section{WEAK DECAYS INTO MULTIPLE TWO-PARTICLE CHANNELS}

\subsection{Multiple-channel extension of the Lellouch-Lüscher formula}

Having found the two channel quantization condition, we are now in a position to work out the two channel generalization of the LL formula which relates weak matrix elements in finite and infinite volume. The derivation follows the original work by Lellouch and Lüscher, Ref. [70], which was extended to a moving frame by Refs. [130, 131].

We begin by introducing a third channel which is decoupled from the original two. This contains a single particle, which we call a $D$-meson, whose mass satisfies

$$
M_{D}>2 M_{\pi}, 2 M_{K}
$$

We next introduce a weak perturbation to the Hamiltonian density

$$
\mathcal{H}(x) \rightarrow \mathcal{H}(x)+\lambda \mathcal{H}_{W}(x)
$$

where $\lambda$ is a parameter which can be varied freely and can, in particular, be taken arbitrarily small. The perturbation $\mathcal{H}_{W}$ is defined to couple channels one and two (pions and kaons) to the third (D-meson) and nothing more. It is convenient to choose it to be invariant under time reversal ( $\mathrm{T}$ ) symmetry. The generalization to perturbations which are not $\mathrm{T}$ invariant is described at the end of the section.

Consider now the finite volume spectrum, first in the absence of the perturbation. The spectrum of two-particle states with $\vec{P}=2 \pi \vec{n}_{P} / L$ is determined by Eq. 2.49). It is $L$ dependent and $L$ can therefore be tuned to make one of the levels, call it $k_{D}$, degenerate with the energy of a single (moving) $D$ meson

$$
E_{k_{D}}=\sqrt{M_{D}^{2}+\vec{P}^{2}}
$$

With $L$ fixed in this way, we now turn on the weak interaction. At leading order in degen- 


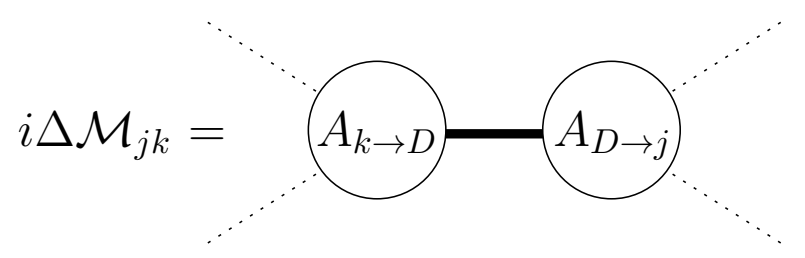

Figure 3.1: The diagram giving rise to the amplitude perturbation $\Delta \mathcal{M}$ [See Eq. [3.9p].

erate perturbation theory this changes the energies to

$$
E^{(1)}=E^{(0)} \pm \lambda V\left|M_{W}\right|
$$

where $V=L^{3}, E^{(0)}=E_{k_{D}}$, and the finite-volume matrix element is

$$
M_{W}={ }_{L}\left\langle k_{D}\left|\mathcal{H}_{W}(0)\right| D\right\rangle_{L}
$$

The subscripts $L$ on the states indicate that they are normalized to unity, unlike the relativistically normalized infinite volume states. Superscripts ${ }^{(1)}$ are used throughout this section to indicate that the quantity includes both the leading order and the $\mathcal{O}(\lambda)$ correction, while superscripts ${ }^{(0)}$ indicate the unperturbed quantity. The effect of the perturbation may also be written in terms of the CM energy as

$$
\begin{aligned}
E^{*(1)} & =M_{D} \pm \lambda \Delta E^{*} \\
\Delta E^{*} & =\frac{E^{(0)} V\left|M_{W}\right|}{M_{D}} .
\end{aligned}
$$

Of course, in addition to affecting the finite volume energy spectrum, the weak perturbation also changes the infinite volume scattering amplitudes. The leading order effect is generated by the diagram of Fig. 3.1, which gives

$$
\mathcal{M}^{(1)}=\mathcal{M}^{(0)} \pm(-\lambda \Delta \mathcal{M})
$$

with

$$
\Delta \mathcal{M}_{j, k}=\frac{\left\langle j\left|\mathcal{H}_{W}(0)\right| D\right\rangle\left\langle D\left|\mathcal{H}_{W}(0)\right| k\right\rangle}{2 E^{(0)} V\left|M_{W}\right|}
$$


The indices $j$ and $k$ run over the two two-particle channels. This perturbation may equivalently be represented through shifts in $\delta_{\alpha}, \delta_{\beta}$ and $\epsilon$

$$
\begin{aligned}
& \delta_{\alpha}^{(1)}\left(E^{*}\right)=\delta_{\alpha}^{(0)}\left(E^{*}\right) \pm \lambda \Delta \delta_{\alpha}\left(E^{*}\right) \\
& \delta_{\beta}^{(1)}\left(E^{*}\right)=\delta_{\beta}^{(0)}\left(E^{*}\right) \pm \lambda \Delta \delta_{\beta}\left(E^{*}\right) \\
& \epsilon^{(1)}\left(E^{*}\right)=\epsilon^{(0)}\left(E^{*}\right) \pm \lambda \Delta \epsilon\left(E^{*}\right),
\end{aligned}
$$

The explicit forms of the perturbed phases are given in Eqs. 3.21)- 3.23 below.

The calculation now proceeds as follows. When the quantities

$$
\delta_{\alpha}^{(0)}\left(E^{*}\right), \delta_{\beta}^{(0)}\left(E^{*}\right), \text { and } \epsilon^{(0)}\left(E^{*}\right)
$$

are placed in the quantization condition [Eq. (2.49)], the condition is satisfied by construction at $E^{*(0)}=M_{D}$. Alternatively if one places

$$
\delta_{\alpha}^{(1)}\left(E^{*}\right), \delta_{\beta}^{(1)}\left(E^{*}\right), \text { and } \epsilon^{(1)}\left(E^{*}\right)
$$

into the same condition then it must be satisfied when evaluated at the perturbed CM energy $E^{*(1)}$, but only to linear order in $\lambda$. The constant order term in the $\lambda$ expansion is just the unperturbed condition, and so it is the vanishing of the $\mathcal{O}(\lambda)$ term that is of interest. The condition that this term vanish gives the relation between finite and infinite volume weak matrix elements that we are after.

The only detail left to discuss, before substituting into the quantization condition and expanding in $\lambda$, is the explicit forms of the amplitude corrections to $\delta_{\alpha}, \delta_{\beta}$ and $\epsilon$. Before these are found it is useful to determine the constraints on the infinite volume matrix elements which arise from Watson's theorem. As shown in App. A, time reversal invariance and unitarity constrain the matrix elements to be such that the following two quantities are real:

$$
\begin{aligned}
& v_{1}=e^{-i \delta_{\alpha}}\left[\sqrt{q_{1}^{*} \eta_{1}} A_{D \rightarrow \pi \pi} \mathrm{c}_{\epsilon}+\sqrt{q_{2}^{*} \eta_{2}} A_{D \rightarrow K K} \mathrm{~s}_{\epsilon}\right], \\
& v_{2}=e^{-i \delta_{\beta}}\left[-\sqrt{q_{1}^{*} \eta_{1}} A_{D \rightarrow \pi \pi} \mathrm{s}_{\epsilon}+\sqrt{q_{2}^{*} \eta_{2}} A_{D \rightarrow K K} \mathrm{c}_{\epsilon}\right] .
\end{aligned}
$$

Here

$$
A_{D \rightarrow \pi \pi} \equiv\left\langle\pi \pi\left|\mathcal{H}_{W}(0)\right| D\right\rangle
$$


and similarly for the $K \bar{K}$ case, normalized so that the decay rates to each channel are

$$
\Gamma_{D \rightarrow j}=\frac{q_{j}^{*} \eta_{j}}{8 \pi M_{D}^{2}}\left|A_{D \rightarrow j}\right|^{2}=\frac{1}{2 M_{D}} P_{j j}^{2}\left|A_{D \rightarrow j}\right|^{2} .
$$

This relation holds also if the particles in a channel are non-degenerate (requiring $\eta=1$ ). All energy-dependent parameters in 3.15 and 3.16 , i.e. $\delta_{\alpha}, \delta_{\beta}, \epsilon$ and $q_{j}^{*}$, are to be evaluated at $E^{*}=M_{D}$.

The results 3.15 and 3.16 hold when the phases of the states are chosen so that the $S$-matrix is symmetric (as is possible given $\mathrm{T}$ invariance). This does not determine the signs of the two matrix elements, and these signs are unphysical. More precisely, the relative sign ambiguity is the same as the ambiguity in the sign of $\epsilon$, so once we have fixed the latter to be positive, the relative sign is physical. The overall sign remains unphysical, and can be chosen, for example to set $v_{1} \geq 0$,

Inverting the relations 3.15 and 3.16 yields

$$
\begin{aligned}
A_{D \rightarrow \pi \pi} & =\frac{1}{\sqrt{q_{1}^{*} \eta_{1}}}\left[v_{1} e^{i \delta_{\alpha}} \mathrm{c}_{\epsilon}-v_{2} e^{i \delta_{\beta}} \mathrm{s}_{\epsilon}\right] \\
A_{D \rightarrow K K} & =\frac{1}{\sqrt{q_{2}^{*} \eta_{2}}}\left[v_{1} e^{i \delta_{\alpha}} \mathrm{s}_{\epsilon}+v_{2} e^{i \delta_{\beta}} \mathrm{c}_{\epsilon}\right] .
\end{aligned}
$$

Inserting these in $\Delta \mathcal{M}$, Eq. (3.9), and using the relation between $\mathcal{M}$ and $S$, Eq. (2.47), and the parametrization of $S$, Eq. (2.48), we find that perturbations to $\delta_{\alpha}, \delta_{\beta}$ and $\epsilon$ are real. This is a consistency check on the calculation (or an alternative derivation of the Watson's theorem constraint). Specifically, we find

$$
\begin{aligned}
\Delta \delta_{\alpha} & =-\mathcal{N} v_{1}^{2} \\
\Delta \delta_{\beta} & =-\mathcal{N} v_{2}^{2} \\
\Delta \epsilon & =-\mathcal{N} \frac{v_{1} v_{2}}{\mathrm{c}_{\alpha} \mathrm{c}_{\beta}\left(\mathrm{t}_{\alpha}-\mathrm{t}_{\beta}\right)}
\end{aligned}
$$

where $\mathrm{t}_{\alpha}=\tan \delta_{\alpha}$, etc., and

$$
\mathcal{N}=\frac{1}{16 \pi E^{(0)} M_{D} V\left|M_{W}\right|} .
$$

We now have all the ingredients to substitute into the quantization condition and determine the LL generalization. We emphasize that, when the expansion in $\lambda$ is performed, $\delta_{\alpha}$, $\delta_{\beta}$ and $\epsilon$ each contribute both from the amplitude corrections of Eqs. (3.21)-(3.23) and from 
the shift in the energy, (3.6). The other contributions arise from the energy dependence of $\phi_{i}=\phi^{P}\left(q_{i}^{*}\right)$. Substituting and simplifying, we find the main result of this section

$$
\mathcal{C}_{1} v_{1}^{2}+\mathcal{C}_{2} v_{2}^{2}+\mathcal{C}_{12} v_{1} v_{2}=\mathcal{C}_{M^{2}}\left|M_{W}\right|^{2}
$$

where

$$
\begin{aligned}
\mathcal{C}_{1}= & \frac{\pi}{16} \frac{\mathrm{t}_{1}+\mathrm{t}_{2}+2 \mathrm{t}_{\beta}+\left(\mathrm{t}_{2}-\mathrm{t}_{1}\right)\left(1-2 \mathrm{~s}_{\epsilon}^{2}\right)}{\mathrm{c}_{\alpha}^{2}} \\
\mathcal{C}_{2}=\frac{\pi}{16} \frac{\mathrm{t}_{1}+\mathrm{t}_{2}+2 \mathrm{t}_{\alpha}+\left(\mathrm{t}_{1}-\mathrm{t}_{2}\right)\left(1-2 \mathrm{~s}_{\epsilon}^{2}\right)}{\mathrm{c}_{\beta}^{2}} & \\
\mathcal{C}_{12}=\frac{\pi}{4}\left(\mathrm{t}_{1}-\mathrm{t}_{2}\right) \frac{\mathrm{s}_{\epsilon} \mathrm{c}_{\epsilon}}{\mathrm{c}_{\alpha} \mathrm{c}_{\beta}} \quad & \frac{\pi^{2} M_{D} V^{2}\left(E^{(0)}\right)^{2}}{2}\left[\frac{\mathrm{t}_{1}^{\prime}}{q_{1}^{*}}\left(\mathrm{t}_{2}+\mathrm{t}_{\beta}+\left(\mathrm{t}_{\alpha}-\mathrm{t}_{\beta}\right) \mathrm{s}_{\epsilon}^{2}\right)+\frac{\mathrm{t}_{2}^{\prime}}{q_{2}^{*}}\left(\mathrm{t}_{1}+\mathrm{t}_{\alpha}+\left(\mathrm{t}_{\beta}-\mathrm{t}_{\alpha}\right) \mathrm{s}_{\epsilon}^{2}\right)\right. \\
& +\frac{4 \mathrm{t}_{\alpha}^{\prime}}{M_{D}}\left(\mathrm{t}_{2}+\mathrm{t}_{\beta}+\left(\mathrm{t}_{1}-\mathrm{t}_{2}\right) \mathrm{s}_{\epsilon}^{2}\right)+\frac{4 \mathrm{t}_{\beta}^{\prime}}{M_{D}}\left(\mathrm{t}_{1}+\mathrm{t}_{\alpha}+\left(\mathrm{t}_{2}-\mathrm{t}_{1}\right) \mathrm{s}_{\epsilon}^{2}\right) \\
& \left.\quad+\frac{4 \mathrm{~s}_{\epsilon}^{2^{\prime}}}{M_{D}}\left(\mathrm{t}_{1}-\mathrm{t}_{2}\right)\left(\mathrm{t}_{\alpha}-\mathrm{t}_{\beta}\right)\right],
\end{aligned}
$$

and where we use the additional notation

$$
\mathrm{t}_{i}=\tan \phi^{P}\left[q_{i}^{*}\right]
$$

All quantities are evaluated at the $D$ mass, and we have dropped the superscript ${ }^{(0)}$. The primes on $\phi_{i}$ indicate derivatives with respect to $q_{i}^{*}$ while those on $\delta_{\alpha}, \delta_{\beta}$ and $\epsilon$ indicate derivatives with respect to $E^{*}$. In each case, these are the natural variables on which the quantities depend. We have checked that this formula reduces to (two copies of) the single-channel LL result if $\epsilon \rightarrow 0$.

We now describe how the result (3.25) can be used in practice. A lattice calculation yields the finite-volume matrix element $\left|M_{W}\right|$, and the aim is to determine the infinitevolume matrix elements $A_{D \rightarrow \pi \pi}$ and $A_{D \rightarrow K K}$. Using the generalized quantization condition 2.49 for three different spectral lines (all chosen to have $E^{*}=M_{D}$ ) one can determine $\delta_{\alpha}$, $\delta_{\beta}$ and $\epsilon$ as described in the previous section. Repeating the procedure at a slightly different energy allows a numerical determination of the required derivatives. One now evaluates $\left|M_{W}\right|$ at the degenerate point on one of the spectral lines. The knowledge of the $S$-matrix 
parameters and their derivatives, together with the value of $L$, allows one to calculate the values of the four $\mathcal{C}$ 's [Eqs. (3.26)-(3.29] ]. Combined with the value of $\left|M_{W}\right|$, one then finds from Eq. 3.25 a quadratic constraint on $v_{1}$ and $v_{2}$. Repeating the procedure for a second spectral line gives an independent constraint, which allows for the determination of $v_{1}$ and $v_{2}$ up to a two-fold ambiguity corresponding to the unknown relative sign. Finally, repeating for a third spectral line resolves the sign ambiguity. With $v_{1}$ and $v_{2}$ determined in this way, one can obtain the infinite-volume matrix elements using Eqs. (3.19) and (3.20). Although this procedure is rather elaborate, we note that (for the case of two channels) three spectral lines are needed both for the determination of the parameters of the $S$-matrix and of the LL factors.

We conclude this section by commenting that Eq. (3.25) factors as

$$
\operatorname{sgn}\left(\mathcal{C}_{1}\right)\left(c_{1} v_{1}+c_{2} v_{2}\right)^{2}=\mathcal{C}_{M^{2}}\left|M_{W}\right|^{2}
$$

where

$$
c_{1}=\sqrt{\left|\mathcal{C}_{1}\right|} \quad c_{2}=\operatorname{sgn}\left[\mathcal{C}_{1} \mathcal{C}_{12}\right] \sqrt{\left|\mathcal{C}_{2}\right|}
$$

The only new information encoded in Eqs. (3.31) and (3.32) relative to Eq. (3.25) is that

$$
4 \mathcal{C}_{1} \mathcal{C}_{2}=\mathcal{C}_{12}^{2}
$$

which can be shown to hold by applying Eq. (2.49) to Eqs. (3.26)-(3.28). Although the factorized form (3.31) is simpler, it does not reduce the number of values of $L$ that are needed because there remains a sign ambiguity (from the square root) at each $L$. What it does make clear, however, is that the generalized LL condition will fail when the signs of $\mathcal{C}_{1}$ and $\mathcal{C}_{M^{2}}$ are opposite. Presumably this cannot happen for physical values of the phase shifts. We stress that this issue also arises in the original one-channel set-up, where the LL formula only makes sense if

$$
\frac{d\left(\delta+\phi^{P}\right)}{d q^{*}}>0
$$

We return to these sign constraints in the next section.

The form 3.31 also allows one to write the LL condition as a factored form in terms of the desired matrix elements,

$$
\left|c_{\pi} A_{D \rightarrow \pi \pi}+c_{K} A_{D \rightarrow K K}\right|^{2}=\left|\mathcal{C}_{M^{2}}\right|\left|M_{W}\right|^{2},
$$


where $c_{\pi}$ and $c_{K}$ are complex, and can be determined from the above results. As this equality holds for any T-invariant form of weak perturbation and for any decay particle, it must imply a relation between finite and infinite volume states

$$
{ }_{L}\left\langle k_{D}\right| \propto c_{\pi}\langle\pi \pi, \text { out }|+c_{K}\langle K \bar{K}, \text { out }|+\ldots
$$

Here the ellipsis indicates the $\pi \pi$ and $K \bar{K}$ states of higher angular momentum which are needed to satisfy the periodic boundary conditions. Indeed, as noted in the original derivation of Ref. [70], the use of the $D$-meson is simply a trick to obtain the normalization factors 1 ] It follows that Eq. (3.35) must also hold for perturbations which are not T-invariant.

The appearance of the linear combination in Eq. (3.36) can be better understood from an alternative derivation of the LL formula, to which we now turn.

\subsection{Alternative derivation of Lellouch-Lüscher formula}

In this section we present a different derivation of the two channel LL relation which has the following advantages: (a) it does not require determining the shifts $\Delta \delta_{\alpha}, \Delta \delta_{\beta}$ and $\Delta \epsilon$, but rather works directly with the change in $\mathcal{M}$; (b) it gives one directly a condition with the factored form, proportional to the left hand side of Eq. (3.35); (c) it allows one to rewrite the LL condition in a simpler form in which the only inputs required are the derivatives of the energies with respect to $L$ along the three spectral lines. This form is likely to be more practical.

We work directly with the condition $\operatorname{det}\left(F^{-1}+i \mathcal{M}\right)=0$, and keep results for general number of channels, $N$, as far as possible. We begin by defining

$$
\begin{aligned}
& \mathcal{X}=F^{-1}+i \mathcal{M} \\
& \mathcal{Y}=S-\left\|e^{-2 i \phi}\right\| .
\end{aligned}
$$

\footnotetext{
${ }^{1}$ In the one-channel case, an alternative line of argument has been developed for obtaining the LL relation, based on matching the density of two-particle states in finite and infinite volumes [148. In the present case, we do not see how to use this approach to determine the relative normalization, $c_{K} / c_{\pi}$, of the two components in the finite volume state. Thus we think that this approach could provide only a consistency check.
} 
and recall from Eq. 2.57) that

$$
\mathcal{X}=P^{-1} \mathcal{Y} P^{-1}
$$

The quantization condition $\operatorname{det} \mathcal{X}=0$ is equivalent to $\mathcal{X}$ having an eigenvector with vanishing eigenvalue. We label this eigenvector $\vec{e}^{X}$. Note also that the symmetry of $\mathcal{X}$ implies $\left(\vec{e}^{X}\right)^{T r}=\overleftarrow{e}^{X}$ is a left eigenvector, also with zero eigenvalue.

Now we can formulate the LL condition in a relatively compact form. As above, let $\mathcal{M}^{(0)}$ be the scattering amplitude at CM energy $E^{*}=M_{D}$. Similarly, let $F^{(0)}$ be the finite-volume factor at this CM energy and for one of the values of box size $L$ for which the quantization condition holds. Then for

$$
\mathcal{X}^{(0)} \equiv\left(F^{(0)}\right)^{-1}+i \mathcal{M}^{(0)},
$$

we have

$$
\overleftarrow{e} X \mathcal{X}^{(0)} \vec{e}^{X}=0
$$

Now, while holding $L$ fixed, we change the energy by $\pm \lambda \Delta E= \pm \lambda V\left|M_{W}\right|$ and change $\mathcal{M}$ to $\mathcal{M}^{(0)} \mp \lambda \Delta \mathcal{M}$, and require that the quantization condition still hold. Thus we have, to linear order in $\lambda$,

$$
\operatorname{det}\left(\mathcal{X}^{(0)}+\lambda \Delta \mathcal{X}\right)=0
$$

where

$$
\Delta \mathcal{X}= \pm\left.\Delta E \frac{\partial \mathcal{X}}{\partial E}\right|_{L} \pm(-i \Delta \mathcal{M})
$$

It follows that there must be a new eigenvector of the form

$$
\vec{e}^{X}+\lambda \Delta \vec{e}^{X}
$$

which is annihilated by the perturbed matrix. From the $\mathcal{O}(\lambda)$ term in

$$
\left[\overleftarrow{e}^{X}+\lambda \Delta \overleftarrow{e} \overleftarrow{e}^{X}\right]\left[\mathcal{X}^{(0)}+\lambda \Delta \mathcal{X}\right]\left[\vec{e}^{X}+\lambda \Delta \vec{e}^{X}\right]=0
$$

we deduce

$$
\overleftarrow{e} \overleftarrow{e}^{X} \Delta \vec{e}^{X}=0
$$

Using the explicit form of $\Delta \mathcal{X}$ this becomes

$$
\left.\Delta E \overleftarrow{e} X \frac{\partial \mathcal{X}}{\partial E}\right|_{L} \vec{e}^{X}=\overleftarrow{e}{ }^{X} i \Delta \mathcal{M} \vec{e}^{X}
$$


where the derivative is evaluated at $E^{*}=M_{D}$.

We can slightly simplify this result by expressing the left hand side in terms of $\mathcal{Y}$ rather than $\mathcal{X}$, and thus removing factors of $P^{-1}$. The point is that, when the derivative acts on the $P^{-1}$ factors in $\mathcal{X}$, the contribution to the left hand side vanishes, since one can still act (either to the left or the right) on the zero-eigenvector. Thus we can rewrite the condition in terms of the zero eigenvector of $\mathcal{Y}$, which is

$$
\vec{e}^{Y}=P^{-1} \vec{e}^{X}
$$

The new form is

$$
\left.\Delta E \overleftarrow{e} Y \frac{\partial \mathcal{Y}}{\partial E}\right|_{L} \vec{e}^{Y}=\overleftarrow{e} X[i \Delta \mathcal{M}] \vec{e}^{X}
$$

We now focus on the $2 \times 2$ case. To proceed, we need the explicit form for $\vec{e}^{Y}$, which is given, up to an overall normalization factor, by

$$
\vec{e}^{Y}=\left[\begin{array}{c}
1 \\
z e^{i\left(\phi_{2}-\phi_{1}\right)}
\end{array}\right]
$$

where $z$ is the real quantity

$$
z=t_{\epsilon} \frac{\sin \left(\delta_{\beta}+\phi_{1}\right)}{\sin \left(\delta_{\beta}+\phi_{2}\right)} .
$$

It is clear from the form of Eq. 3.49 and the relation 3.48 that the normalization of $\vec{e}^{Y}$ is irrelevant and so we have chosen a relatively simple unnormalized form.

We evaluate the right hand side of Eq. (3.49) using the form of $\Delta \mathcal{M}$, Eq. (3.9). It is immediately apparent that the result factorizes, given that $\Delta \mathcal{M}$ is an outer product. This will hold for all $N$. In the $N=2$ case we have

$$
\overleftarrow{e}^{X}[i \Delta \mathcal{M}] \vec{e}^{X}=i e^{-2 i \phi_{1}} \frac{M_{\infty}^{2}}{8 \pi M_{D} E^{(0)} V\left|M_{W}\right|}
$$

where

$$
M_{\infty}=e^{i \phi_{1}} \sqrt{q_{1}^{*} \eta_{1}} A_{D \rightarrow \pi \pi}+z e^{i \phi_{2}} \sqrt{q_{2}^{*} \eta_{2}} A_{D \rightarrow K K}
$$

Here we have used the assumed T-invariance of $\mathcal{H}_{W}$. We have pulled out the phase $e^{-2 i \phi_{1}}$ so that $M_{\infty}$ is real. Its reality is not obvious, but can be established using the results derived 
from Watson's theorem and given in App. A. In particular, an algebraic exercise shows that

$$
M_{\infty}=\sin \left(\phi_{1}-\phi_{2}\right)\left[-v_{1} \frac{c_{\epsilon}}{\sin \left(\delta_{\alpha}+\phi_{2}\right)}+v_{2} \frac{s_{\epsilon}}{\sin \left(\delta_{\beta}+\phi_{2}\right)}\right] \text {, }
$$

and we recall that the quantities $v_{1}$ and $v_{2}$ [defined above in Eqs. (3.15)-(3.16)] are real.

The result (3.53) makes clear that, for any choice of $\mathcal{H}_{W}$, one ends up with the matrix element to a given (complex) linear combination of $\langle\pi \pi|$ and $\langle K \bar{K}|$ states, since all the factors $\left(\phi_{1}, \phi_{2}\right.$ and $\left.z\right)$ are determined by $E^{(0)}$ and $L$. Indeed, what the LL method has allowed us to do is determine the coefficients of the s-wave $\langle\pi \pi|$ and $\langle K \bar{K}|$ components within the finite-volume state. As mentioned above, this decomposition has nothing to do with $\mathcal{H}_{W}$, and thus we can use the result for any $\mathcal{H}_{W}$, including one involving T-violation. By comparing the result (3.53) to the general decomposition of the finite-volume state, Eq. (3.36), we can read off the ratio of the coefficients,

$$
\frac{c_{K}}{c_{\pi}}=e^{i\left(\phi_{2}-\phi_{1}\right)} z \sqrt{\frac{q_{2}^{*} \eta_{2}}{q_{1}^{*} \eta_{1}}} .
$$

It is interesting that the relative phase between $c_{K}$ and $c_{\pi}$ is determined by the kinematic phases $\phi_{j}$. Given the form of $\Delta \mathcal{M}$, and the fact that, in Eq. $(3.52)$, it is sandwiched between $\overleftarrow{e}^{X}$ and $\vec{e}^{X}$, it follows that the zero eigenvector itself gives the relative size of the $\pi \pi$ and $K \bar{K}$ contributions:

$$
\vec{e}^{X} \propto\left(\begin{array}{c}
c_{\pi} \\
c_{K}
\end{array}\right) .
$$

This illustrates in a direct way that the linear combination which appears is completely independent of the form of $\mathcal{H}_{W}$, since the eigenvector of $\mathcal{X}$ knows nothing about this perturbation.

Having discussed the right hand side of Eq. 3.49 in some detail we now turn to the left. Specifically, we show that it is possible to write the left-hand side in terms of the derivative of the spectral energy with respect to $L$. To motivate this form, we first recall that the LL result of the previous section depends on $\delta_{\alpha}, \delta_{\beta}$ and $\epsilon$ and their derivatives, evaluated at $E^{*}=M_{D}$. As described in Sec. 3.1, the three $S$-matrix parameters may be determined, using Eq. 2.49), by finding three different pairs $\left\{L, \vec{n}_{P}\right\}$ for which there is a spectral line 
$E_{k}\left(L ; \vec{n}_{P}\right)$ satisfying $E_{k}^{*}\left(L ; \vec{n}_{P}\right)=M_{D}$ [see Eq. (2.50)]. Furthermore, by slightly changing the three $L$ values, one can determine $\delta_{\alpha}, \delta_{\beta}$ and $\epsilon$ at slightly different energies and thus deduce the derivatives at $M_{D}$.

The point of reiterating these steps is to note that, since the lattice simulation actually gives the energy spectrum as a function of $L$, it would be preferable if the LL result could be rewritten directly in terms of the properties of the spectrum. In this way the extra step of separately working out the phase shifts and their derivatives would be avoided. This turns out to be possible, as we now show.

We use the quantization condition in the form $\operatorname{det} \mathcal{Y}=0$. To stay on a spectral line $E_{k}\left(L ; \vec{n}_{P}\right)$ as we vary $E$ away from the moving frame $D$-meson energy $E^{(0)}$, we need to vary $L$ in such a way that this condition remains fulfilled. We note that, while $F$ depends on both $E^{*}$ and $L, S$ depends only on $E^{*}$. Thus we use $E^{*}$ and $L$ as independent variables. Then the condition to stay on a spectral line becomes

$$
0=\overleftarrow{e} Y\left[\left.\Delta E^{*} \frac{\partial \mathcal{Y}}{\partial E^{*}}\right|_{L}+\left.\Delta L \frac{\partial \mathcal{Y}}{\partial L}\right|_{E^{*}}\right] \vec{e}^{Y}
$$

which leads to

$$
\left.\frac{d E_{k}^{*}}{d L}\right|_{\text {line }}=-\frac{\overleftarrow{e} Y \frac{\partial \mathcal{Y}}{\partial L} \vec{e}^{Y}}{\overleftarrow{e} Y \frac{\partial \mathcal{Y}}{\partial E^{*}} \vec{e}^{Y}}
$$

Here, in the left-hand side, the subscript "line" indicates that the derivative is along a spectral line with fixed $\vec{n}_{P}$.

The key features of Eq. (3.58) are that the denominator on the right-hand side is, up to a simple overall factor, equal to the quantity appearing on the left hand side of the Eq. (3.49), while the numerator is a kinematic factor. Specifically, using

$$
\overleftarrow{e} Y \frac{\partial \mathcal{Y}}{\partial E^{*}} \vec{e}^{Y}=\frac{E^{*}}{E} \overleftarrow{e} Y \frac{\partial \mathcal{Y}}{\partial E} \vec{e}^{Y}
$$

and

$$
\left.\frac{d E_{k}}{d L}\right|_{\text {line }}=\left.\frac{E_{k}^{*}}{E_{k}} \frac{d E_{k}^{*}}{d L}\right|_{\text {line }}-\frac{\vec{P}^{2}}{E L}
$$

(which follows since $E^{2}=\left(E^{*}\right)^{2}+(\vec{P} L)^{2} / L^{2}$ and because $\vec{P} L$ is fixed along the spectral line), we find

$$
\overleftarrow{e} Y \frac{\partial \mathcal{Y}}{\partial E} \vec{e}^{Y}=-2 i e^{-2 i \phi_{1}} \frac{\frac{\partial \phi_{1}}{\partial L}+z^{2} \frac{\partial \phi_{2}}{\partial L}}{\left.\frac{d E_{k}}{d L}\right|_{\text {line }}+\frac{\vec{P}^{2}}{E L}}
$$


Combining this with 3.49 and 3.52 we conclude

$$
\frac{M_{\infty}^{2}}{16 \pi M_{D} E^{(0)} V^{2}\left|M_{W}\right|^{2}}=-\frac{\frac{\partial \phi_{1}}{\partial L}+z^{2} \frac{\partial \phi_{2}}{\partial L}}{\left.\frac{d E_{k}}{d L}\right|_{\text {line }}+\frac{\vec{P}^{2}}{E^{(0)} L}} .
$$

We thus have found an alternative form of the LL relation which is simpler than Eq. (3.25), and also likely to be more practical.

The single channel version of (3.62) is instructive. It can be written, using Eq. 3.18), in terms of the decay rate:

$$
\Gamma_{D \rightarrow \pi \pi}=\frac{2 E^{(0)} V^{2}\left|M_{W}\right|^{2}}{M_{D}}\left[\frac{-\frac{\partial \phi}{\partial L}}{\left.\frac{d E_{k}}{d L}\right|_{\text {line }}+\frac{\vec{P}^{2}}{E^{(0)} L}}\right] .
$$

This form holds both for identical and non-identical particles, with the symmetry factor being contained in $\Gamma$. It also sheds light on the sign constraints discussed in the previous section. The right-hand side must be positive. Based on numerical studies, we find that $\partial \phi / \partial L$ is always positive, implying that the denominator, which is proportional to $d E_{k}^{*} / d L$, must be negative.

The same holds for the two-channel result, Eq. (3.62). In order for the right-hand side to be positive, the denominator must be negative. Since we could do the LL analysis on almost any spectral line, this appears to imply that $d E_{k}^{*} / d L<0$ in general. The only exception is for a state with $E_{k}^{*}$ below the two particle threshold. Such a state occurs, for example, as the lowest energy state for $\vec{P}=0$ if there is an attractive interaction. For such a state one has $d E_{k}^{*} / d L=d E_{k} / d L>0$, i.e. of the "wrong" sign. But in this case the LL analysis does not apply, because the particle lies below threshold in infinite volume. 


\section{Chapter 4}

\section{ONE-TO-TWO TRANSITION MATRIX ELEMENTS1}

In order to determine transition matrix elements, both two- and three-point correlation functions are needed. From two-point correlation functions one can most easily extract the finite-volume spectrum. From appropriate ratios of two- and three-point correlation functions one may also obtain finite-volume matrix elements of external currents. In Section 4.1, we present a derivation of the finite-volume two-point correlation functions for one and two-particle systems. ${ }^{2}$ In this analysis, we consider an arbitrary number of two-particle channels which mix with arbitrarily strong couplings. We restrict attention to spin-zero particles, but do formally accommodate all two-particle angular-momenta states. From the two-point correlators of such systems one can obtain expressions for the one and two-particle finite-volume spectrum. The finite-volume corrections to the masses of single particles are exponentially suppressed in $m_{\pi} L$, where $L$ is the spatial extent of the finite volume and $m_{\pi}$ is the pion mass [141]. As long as one requires $m_{\pi} L \gtrsim 4$, then the finite-volume corrections to the masses are percent level. We neglect such exponentially suppressed corrections throughout.

In contrast to single-particle states, the finite-volume energy spectrum above two-particle threshold cannot be directly identified with infinite-volume observables. The spectrum does however encode information about the infinite-volume on-shell scattering amplitude. The formalism responsible for connecting the finite-volume spectrum with scattering information is known as the Lüscher method [127, 128]. This approach has been investigated and generalized in various contexts [129, 150, 151, 152, 153, 154, 155, 131, 130, 156, 157, 143,

\footnotetext{
${ }^{1}$ This chapter is taken from Ref. [149]

${ }^{2}$ Although it is customary in the literature to label correlation functions by the total number of particles in the initial and final state, since the number of particles is not a well-defined quantity in finite-volume we choose to simply refer to correlation functions that have no insertion of external currents as two-point correlation functions and those that do have an external current insertion as three-point correlation functions.
} 
145, 158, 159, 160, 161, 162, 163, 132, 140, 146, 164, 165, 134, 166] including most recently a method for describing all $\mathbf{2} \rightarrow \mathbf{2}$ systems with arbitrary quantum numbers, open channels and boundary conditions [167]. There have also been attempts to generalize this formalism for three-particle systems [137, 138, 139, 168, but a general solution for the three body system in a finite volume has not been found. Finally, in the energy regime of elastic scattering, the formalism has been extensively implemented in numerical lattice simulations. See for example Refs. [169, 170, 171, 172, 173, 174, 175, 176, 177, 178, 179, 180, 181, 182, 183, 184, 185, 186, 187, 188.

Section 4.1 of this work recovers the well-know quantization condition for a system with any number of two-scalar channels, with arbitrary angular momentum as well as total linear momenta. The result is

$$
\operatorname{det}\left[\mathbb{M}\left(E_{n}\right)\right]=\operatorname{det}\left[\mathbb{K}\left(E_{n}\right)+\left(\mathbb{F}^{V}\left(E_{n}\right)\right)^{-1}\right]=0,
$$

and was first obtained in Refs. [140, 146]. Here $\mathbb{K}$ is the two-particle K-matrix (defined in Eq. 4.39 and related to the scattering amplitude via Eq. 4.41) and $\mathbb{F}^{V}$ is a volume-dependent kinematic matrix (defined in Eq. 4.30). Both of these are matrices over angular momenta as well as all open two-particle channels, and the determinant is understood to act on this direct-product space. Until recently, the only numerical implementation of the coupledchannel formalism was by Guo in an exploratory numerical calculation of a two-channel system in $1+1$ dimensional lattice model [189]. The first application of this formalism in a lattice QCD calculation was by the Hadron Spectrum collaboration in a benchmark calculation of the $\pi K-K \eta$ systems [92, which unambiguously demonstrates that coupledchannel systems can be studied via lattice QCD.

In Section 4.2 of the paper, we derive a non-perturbative relation for the relativistic three-point correlation function. We first review how currents can be properly subduced onto irreps of the finite-volume symmetry groups. Having defined the subduced current, we proceed to evaluate the three-point function corresponding to a process $\varphi_{1} \rightarrow\left\{\varphi_{2} \varphi_{3}, \varphi_{4} \varphi_{5}, \ldots\right\}$, where $\varphi_{i}$ label single-scalar states. For systems where the initial and/or final states have overlap with multi-particle states the relationship between the finite-volume matrix element of a current and its infinite volume analog is not obvious. This was fist pointed out by Lel- 
louch and Lüscher in the context of $K \rightarrow \pi \pi$ weak decays [70]. In their seminal work, they showed that the absolute value of the transition matrix element in finite-volume is proportional to the physical transition matrix element, with a proportionally factor that depends on the S-wave phase shift of the final state. This proportionality factor is commonly referred to as the $L L$-factor. This formalism has been extended to systems with nonzero total momentum [130, 131, 190], systems with multiple strongly coupled two-scalar channels [140], $\pi^{0} \rightarrow \gamma \gamma[191], N \gamma \rightarrow N \pi[136]^{3}$ as well as $\mathbf{2} \rightarrow \mathbf{2}$ processes that are mediated by the weak interaction [153, 123, 192, 146]. See Refs. [193, 194, 195, 88, for recent examples of the implementation of this formalism on LQCD calculations of the $K \rightarrow \pi \pi$ decay amplitude. 4 .

In this work we generalize the Lellouch-Lüscher result by allowing the current to insert arbitrary momentum and energy to the system. We restrict ourselves to an initial state that transforms as either a scalar or a pseudoscalar but consider final states in any irrep of the relevant finite-volume symmetry group. As already mentioned, we also consider multiple strongly-coupled two-particle states. Within this generic framework, we find a master equation that relates the finite-volume matrix elements of currents with the physically relevant infinite-volume counterpart

$$
\begin{aligned}
&\left|\left\langle E_{\Lambda_{f}, n_{f}} \mathbf{P}_{f} ; L\left|\widetilde{\mathcal{J}}_{\Lambda \mu}^{[J, P,|\lambda|]}\left(0, \mathbf{P}_{f}-\mathbf{P}_{i}\right)\right| E_{\Lambda_{i}, 0} \mathbf{P}_{i} ; L\right\rangle\right| \\
&=\frac{1}{\sqrt{2 E_{\Lambda_{i}, 0}}} \sqrt{\left[\mathcal{A}_{\Lambda_{f}, n_{f} ; \Lambda \mu}^{\dagger} \mathcal{R}_{\Lambda_{f}, n_{f}} \mathcal{A}_{\Lambda_{f}, n_{f} ; \Lambda \mu}\right]},
\end{aligned}
$$

where $\widetilde{\mathcal{J}}_{\Lambda \mu}^{[J, P,|\lambda|]}\left(0, \mathbf{P}_{f}-\mathbf{P}_{i}\right)$ is a current whose quantum numbers and labels are thoroughly defined in Section 4.2.1. $\left|E_{\Lambda_{i}, 0} \mathbf{P}_{i} ; L\right\rangle$ and $\left|E_{\Lambda_{f}, n_{f}} \mathbf{P}_{f} ; L\right\rangle$ respectively denote the initial and

\footnotetext{
${ }^{3}$ In the production of this manuscript a similar and independent work by A. Agadjanov, et al., appeared in the literature 136. In their work, the authors considered pion-photoproduction off a nucleon, $N \gamma \rightarrow N \pi$ in the non-relativistic limit. The authors demonstrated how to study transitions amplitudes for systems with nonzero intrinsic spin. In doing so, they restrict the final two-particle state to be at rest and neglect corrections due to partial wave mixing, but they do allow for the finite volume of the systems to have an asymmetry along one of the Cartesian axes.

${ }^{4}$ For historically relevant theoretical and numerical developments regarding nonleptonic weak decay on the lattice see Refs. 196, 197, 198, 199, 200, 201, 202, 190, 203, 204, 205, 206, 207, 208, 209, 210, 211, 212, 213, 214, 215, 216].
} 
final finite-volume states; the former has the energy and the quantum numbers of a single particle while the latter has that of two particles. Our result relates this finite-volume matrix element to

$$
\left\langle a, P_{f}, J m_{J} ; \infty\left|\widetilde{\mathcal{J}}_{\Lambda \mu}^{[J, P,|\lambda|]}(0, \mathbf{Q} ; \infty)\right| P_{i} ; \infty\right\rangle=\left[\mathcal{A}_{\Lambda \mu ; J m_{J}}\right]_{a}(2 \pi)^{3} \delta^{3}\left(\mathbf{P}_{f}-\mathbf{P}_{i}-\mathbf{Q}\right)
$$

were $a$ is a channel index denoting the two particle flavors in the asymptotic state. In Eq. 4.2, $\mathcal{A}$ is understood as a column vector (and $\mathcal{A}^{\dagger}$ a row) in the combined angularmomentum/channel space. Finally $\mathcal{R}_{\Lambda_{f}, n_{f}}$, defined in Eq. 4.104 below, is a matrix in the same space that depends only on the strong-interaction as well as the linear extent of the finite volume. It is the coupled-channel and arbitrary-angular-momentum generalization of the LL-factor. On all quantities the subscript $\Lambda_{f}$ indicates that angular-momentum space has been projected onto a particular finite-volume irrep, and $n_{f}$ is an integer labeling the finite-volume level considered.

Just like the quantization condition of the two-particle spectrum, the master equation for finite-volume matrix elements can be significantly simplified by considering specific examples. In order to illustrate how this is done, in Section 4.2.4 we consider several examples. First, Section 4.2 .4 demonstrates that in the limit that the current considered is a pseudoscalar that injects zero total momentum, the master equation reproduces the well known result of $K \rightarrow \pi \pi$ [70, 130, 131, 190]. In Section 4.2.4 we consider the matrix element for $\pi \gamma \rightarrow \pi \pi \rightarrow \rho$. For this case the two hadrons in the final states are exactly degenerate and therefore odd and even partial waves do not mix, even when the system is boosted. Parity and angular momentum conservation requires that the final state cannot have overlap with an S-wave. Therefore the final state is in a $\mathrm{P}$-wave with leading order contamination from the F-wave. By neglecting this contamination, we obtain an explicit expression for the P-wave LL-factor for such a system, and find large volume deviation from the well known S-wave result. For processes where the final states are composed of nondegenerate particles, odd and even partial waves will in general mix. Furthermore, in general all strongly-coupled channels that can go on-shell will be present in the final state. Even in the example above for sufficiently high energies we must consider $\pi \gamma \rightarrow\{\pi \pi, K \bar{K}, \ldots\}$. With these complexities in mind, in Section 4.2 .4 we discuss the implication of the master 
equation for systems with coupled channels, regardless of whether the mixing in an infinite volume effect (e.g., $\pi \pi-K \bar{K}$ ) or a finite volume artifact (e.g., S and P-wave mixing). In Section 4.2.4 we demonstrate how this result reproduces previously known relation for $D \rightarrow\{\pi \pi, K \bar{K}\}[140]:^{5}$

Although it is most convenient to perform LQCD calculation using periodic boundary conditions in a cubic volume, one may also run simulations with twisted boundary conditions (TBCs) [217, 154] in a volume that is an arbitrary rectangular prism. Ref. [167] showed how to compactly incorporate all of these scenarios into a single generic result. For completeness Appendix $\mathrm{C}$ reviews how the results presented here can be implemented for volumes with generic geometry and twisted boundary conditions.

\subsection{Two-point correlation functions}

In this section we derive expressions for the one-particle and two-particle two-point correlation functions in a finite volume. To achieve this we must first define appropriate interpolating operators. These are most conveniently classified according to the irreducible representations (irreps) of the relevant symmetry group. For a system at rest in a finite cubic volume, the symmetry group is the octahedral group, $O_{h}$. In order to accommodate systems with half-integer spin, one has to consider the double cover of the octahedral group, denoted by $O_{h}^{D}$ [218]. For systems in flight with total momentum $\mathbf{P}$, the symmetry is reduced to a subgroup of $O_{h}$ or $O_{h}^{D}$, defined by the subset of octahedral transformations which leave $\mathbf{P}$ invariant. This is referred to as a little group and will be labeled $\operatorname{LG}(\mathbf{P})$.

Let $\varphi_{\Lambda \mu}\left(x_{0}, \mathbf{P}\right)$ denote a single particle interpolating operator at Euclidean time $x_{0}$ with momentum $\mathbf{P}$ and in row $\mu$ of the $\Lambda$ irrep of $\operatorname{LG}(\mathbf{P}){ }^{6}$ Because $\Lambda \mu$ are good quantum numbers in finite volume, the one-particle two-point functions will not mix states in different rows or irreps

$$
C_{\Lambda^{\prime} \mu^{\prime}, \Lambda \mu}^{(1)}\left(x_{0}-y_{0}, \mathbf{k}\right) \equiv\left\langle 0\left|\varphi_{\Lambda^{\prime} \mu^{\prime}}\left(x_{0}, \mathbf{k}\right) \varphi_{\Lambda \mu}^{\dagger}\left(y_{0},-\mathbf{k}\right)\right| 0\right\rangle \propto \delta_{\Lambda^{\prime}, \Lambda} \delta_{\mu^{\prime}, \mu}
$$

\footnotetext{
${ }^{5}$ In both the present work and [140] the formalism neglects coupling to states with more than two particles.

${ }^{6}$ For details regarding the construction of these operators from quark and gluonic degrees of freedom we direct the readers to Refs. [219, 220, 221, 156, 222, 223, 224, 225, 226, 158] and references therein.
} 
In this study, we will focus on the scenario where the single-particle states are either pseudoscalars or scalars.7 In such cases there is a single one-dimensional irrep that has overlap with the particle of interest, and the irrep is exclusively specified by its momentum. For example, as shown explicitly in Tables $4.1(\mathrm{a}) \&(\mathrm{~b})$, the pseudoscalar mesons are in the $\mathbb{A}_{1}^{-}$irrep of $O_{h}$ when at rest and in the $\mathbb{A}_{2}$ irrep of $L G(\mathbf{k})$ when in flight. Therefore, it is sufficient to define the single particle interpolating operators in terms of their momenta and we will drop the $\Lambda \mu$ subscript. We thus introduce

$$
\begin{array}{r}
C^{(1)}\left(x_{0}-y_{0}, \mathbf{k}\right) \equiv\left\langle 0\left|\varphi\left(x_{0}, \mathbf{k}\right) \varphi^{\dagger}\left(y_{0},-\mathbf{k}\right)\right| 0\right\rangle=e^{-E_{k}^{(1)}\left(x_{0}-y_{0}\right)}\left|\left\langle 0|\varphi(0, \mathbf{k})| E^{(1)} \mathbf{k} ; L\right\rangle\right|^{2} \\
+\mathcal{O}\left(L^{3} e^{-E_{3, t h}^{(1)}\left(x_{0}-y_{0}\right)} / E_{3, t h}^{(1)}\right)
\end{array}
$$

where $L$ is the linear extent of the finite cubic spatial volume and $E_{k}^{(1)}, E_{3, t h}^{(1)}$ denote the lowest two eigenvalues of the moving-frame Hamiltonian, in the subspace that has overlap with $\langle 0| \varphi_{\Lambda \mu}(0, \mathbf{k})$. We have assumed $x_{0}>y_{0}$ to order the operators before inserting a complete set of states. As the subscript suggests, in QCD the first excited energy $E_{3, t h}^{(1)}$ corresponds to a state in the vicinity of the three-particle threshold.

One can also calculate the correlation function's leading time dependence directly from the fully dressed single particle propagator (see Fig. 4.1(c)

$$
\begin{aligned}
C^{(1)}\left(x_{0}-y_{0}, \mathbf{k}\right) & =L^{3} \int \frac{d P_{0}}{2 \pi}\left(\frac{1}{2 \omega_{\mathbf{k}}\left(i P_{0}+\omega_{\mathbf{k}}\right)}+\cdots\right) e^{i P_{0}\left(x_{0}-y_{0}\right)} \\
& =L^{3} \frac{e^{-\omega_{\mathbf{k}}\left(x_{0}-y_{0}\right)}}{2 \omega_{\mathbf{k}}}+\mathcal{O}\left(L^{3} e^{-E_{3, t h}^{(1)}\left(x_{0}-y_{0}\right)} / E_{3, t h}^{(1)}\right),
\end{aligned}
$$

where $\omega_{\mathbf{k}}=\sqrt{m^{2}+\mathbf{k}^{2}}$, with $m$ equal to the physical infinite-volume pole mass. In the first line, the ellipses denote corrections that are finite at the single particle pole. This includes terms with poles at higher values of imaginary $P_{0}$ which correspond to higher energy states.

\footnotetext{
${ }^{7}$ For QCD near the physical point there are no stable scalar particles, only pseudoscalar mesons. At unphysical quark masses, by contrast, one finds stable scalar particles as well. Additionally, although LQCD is the motivation for this work, this formalism is model independent and is relevant for studying hadronic physics as well as atomic physics in a finite volume. (See Refs. 227, 228] and references within for examples of atomic physics calculations performed in a finite volume.) See Ref. [136] for insight into how to deal with states with nonzero spin in the non-relativistic limit.
} 
(a)

\begin{tabular}{c|c|c|c} 
Group & $J^{P}$ & $\Lambda(\mu)$ & {$\left[C_{\Lambda}^{J}\right]_{\mu, \lambda}$} \\
\hline$O_{h}^{D}$ & $0^{ \pm}$ & $\mathbb{A}_{1}^{ \pm}(1)$ & 1 \\
$\frac{\mathbf{Q} L}{2 \pi}=(0,0,0)$ & $1^{ \pm}$ & $\mathbb{T}_{1}^{ \pm}(1)$ & $\delta_{1, \lambda}$ \\
& $1^{ \pm}$ & $\mathbb{T}_{1}^{ \pm}(2)$ & $\delta_{0, \lambda}$ \\
& $1^{ \pm}$ & $\mathbb{T}_{1}^{ \pm}(3)$ & $\delta_{-1, \lambda}$ \\
& $2^{ \pm}$ & $\mathbb{T}_{2}^{ \pm}(1)$ & $\delta_{1, \lambda}$ \\
& $2^{ \pm}$ & $\mathbb{T}_{2}^{ \pm}(2)$ & $\left(\delta_{2, \lambda}-\delta_{-2, \lambda}\right) / \sqrt{2}$ \\
& $2^{ \pm}$ & $\mathbb{T}_{2}^{ \pm}(3)$ & $\delta_{-1, \lambda}$ \\
& $2^{ \pm}$ & $\mathbb{E}^{ \pm}(1)$ & $\delta_{0, \lambda}$ \\
& $2^{ \pm}$ & $\mathbb{E}^{ \pm}(2)$ & $\left(\delta_{2, \lambda}+\delta_{-2, \lambda}\right) / \sqrt{2}$ \\
\hline
\end{tabular}

(b)

\begin{tabular}{c|c|c|c}
$\mathrm{LG}(\mathbf{Q})$ & $|\lambda|^{\tilde{\eta}}$ & $\Lambda(\mu)$ & $\mathcal{S}_{\Lambda \mu}^{\tilde{\eta}, \lambda}$ \\
\hline $\operatorname{Dic}_{4}$ & $0^{+}$ & $\mathbb{A}_{1}(1)$ & 1 \\
$\frac{\mathbf{Q} L}{2 \pi}=(0,0, n)$ & $0^{-}$ & $\mathbb{A}_{2}(1)$ & 1 \\
& 1 & $\mathbb{E}(1)$ & $\left(\delta_{s,+}+\tilde{\eta} \delta_{s,-}\right) / \sqrt{2}$ \\
& 1 & $\mathbb{E}(2)$ & $\left(\delta_{s,+}-\tilde{\eta} \delta_{s,-}\right) / \sqrt{2}$ \\
& 2 & $\mathbb{B}_{1}(1)$ & $\left(\delta_{s,+}+\tilde{\eta} \delta_{s,-}\right) / \sqrt{2}$ \\
& 2 & $\mathbb{B}_{2}(1)$ & $\left(\delta_{s,+}-\tilde{\eta} \delta_{s,-}\right) / \sqrt{2}$ \\
\hline $\operatorname{Dic}_{2}$ & $0^{+}$ & $\mathbb{A}_{1}(1)$ & 1 \\
$\frac{\mathbf{Q} L}{2 \pi}=(n, n, 0)$ & $0^{-}$ & $\mathbb{A}_{2}(1)$ & 1 \\
& 1 & $\mathbb{B}_{1}(1)$ & $\left(\delta_{s,+}+\tilde{\eta} \delta_{s,-}\right) / \sqrt{2}$ \\
& 1 & $\mathbb{B}_{2}(1)$ & $\left(\delta_{s,+}-\tilde{\eta} \delta_{s,-}\right) / \sqrt{2}$ \\
\hline $\operatorname{Dic}_{3}$ & 2 & $\mathbb{A}_{1}(1)$ & $\left(\delta_{s,+}+\tilde{\eta} \delta_{s,-}\right) / \sqrt{2}$ \\
$\frac{\mathbf{Q} L}{2 \pi}=(n, n, n)$ & 2 & $\mathbb{A}_{2}(1)$ & $\left(\delta_{s,+}-\tilde{\eta} \delta_{s,-}\right) / \sqrt{2}$ \\
& $0^{+}$ & $\mathbb{A}_{1}(1)$ & 1 \\
& $0^{-}$ & $\mathbb{A}_{2}(1)$ & 1 \\
& 1 & $\mathbb{E}(1)$ & $\left(\delta_{s,+}+\tilde{\eta} \delta_{s,-}\right) / \sqrt{2}$ \\
& 1 & $\mathbb{E}(2)$ & $\left(\delta_{s,+}-\tilde{\eta} \delta_{s,-}\right) / \sqrt{2}$ \\
& 2 & $\mathbb{E}(1)$ & $\left(\delta_{s,+}-\tilde{\eta} \delta_{s,-}\right) / \sqrt{2}$ \\
& 2 & $\mathbb{E}(1)$ & $-\left(\delta_{s,+}-\tilde{\eta} \delta_{s,-}\right) / \sqrt{2}$ \\
\hline
\end{tabular}

Table 4.1: (a) Shown are the subduction coefficients, $\left[C_{\Lambda}^{J}\right]_{\mu, \lambda}$ used to project states onto the irreps of $O_{h}^{D}$ for integer spin particles. (b) Shown are the subduction coefficients determined in Ref. [226], for $|\lambda| \leq 2$, where $s=\operatorname{sign}(\lambda)$ and $\widetilde{\eta}=(-1)^{l+J}$ used to project operators onto the irreps of the Dic 4 , Dic 2 , and Dic 3 groups as shown in Eq. 4.79. 
We emphasize that, in arriving at this identity, we have used the on-shell renormalization convention in which the residue of the single particle propagator is set to 1 . This convension is equivalently expressed as

$$
\left\langle 0|\phi(0, \mathbf{0})| E^{(1)} \mathbf{k} ; \infty\right\rangle=1
$$

where $\phi\left(x_{0}, \mathbf{x}\right)$ is the Fourier transform of $\varphi\left(x_{0}, \mathbf{k}\right)$ and $\left|E^{(1)} \mathbf{k} ; \infty\right\rangle$ is the infinite-volume one-particle state with relativistic normalization

$$
\left\langle E^{(1)} \mathbf{k}^{\prime} ; \infty \mid E^{(1)} \mathbf{k} ; \infty\right\rangle=2 \omega_{\mathbf{k}}(2 \pi)^{3} \delta^{3}\left(\mathbf{k}^{\prime}-\mathbf{k}\right) .
$$

By comparing Eqs. $4.5 \& 4.6$, we deduce $E_{k}^{(1)}=\omega_{\mathbf{k}}$ and

$$
\left|\left\langle 0|\varphi(0, \mathbf{k})| E^{(1)} \mathbf{k} ; L\right\rangle\right|=\sqrt{\frac{L^{3}}{2 \omega_{\mathbf{k}}}} .
$$

These relations hold up to exponentially suppressed corrections of the form $e^{-m L}$, which we discuss in more detail below. We stress that Eq. 4.9 is only a statement of renormalization convention on $\varphi$ together with the normalization convention for finite-volume states

$$
\left\langle E^{(1)} \mathbf{k} ; L \mid E^{(1)} \mathbf{k} ; L\right\rangle=1 .
$$

As will become evident in Section 4.2 , the choice of residue equal to one does not impact the final result, Eq. 4.2. Any other choice for the residue would exactly cancel. The motivation for deriving Eq. 4.9 in the manner just presented is that it provides a straightforward warmup for our analysis of the two-particle two-point correlation function, to which we now turn.

The two-particle correlation function can be determined by considering an alternative energy range and using two- instead of one-particle interpolating fields. For the sake of generality, we consider a system with $\mathrm{N}$ coupled two-particle channels. We label the masses in the $j$ th channel $m_{j, 1}$ and $m_{j, 2}$, with $m_{j, 1} \leq m_{j, 2}$. We continue to restrict our attention to spin zero particles. The particles in the $j t h$ channel can go on-shell if the c.m. energy $E^{*}$ satisfies $m_{j, 1}+m_{j, 2} \leq E^{*}<E_{t h}^{*}$. Here $E_{t h}^{*}$ is the energy of the first allowed multi-particle threshold, boosted to the c.m. frame 8 In practice we must require $E^{*} \ll E_{t h}^{*}$, because if

\footnotetext{
${ }^{8}$ For a system with G-parity, e.g., $\pi \pi$, this corresponds to the four-particle threshold, for any other
} system this corresponds to the three-particle threshold. 
$E^{*}$ is too close to the multi-particle threshold then the neglected exponentially suppressed corrections become enhanced.

The on-shell c.m. relative momentum for the $j t h$ channel satisfies

$$
k_{j, \text { on }}^{* 2}=\frac{E^{* 2}}{4}-\frac{\left(m_{j, 1}^{2}+m_{j, 2}^{2}\right)}{2}+\frac{\left(m_{j, 1}^{2}-m_{j, 2}^{2}\right)^{2}}{4 E^{* 2}} .
$$

Functions and coordinates evaluated in the c.m. frame will always have a superscript "*", and it important to remember that a function $f$ in a moving frame that depends on $k$ can always be related to the c.m. frame function $f^{*}$ via $f^{*}\left(k^{*}\right) \equiv f(k)$. This just defines a coordinate change and does not imply anything about the Lorentz representation of $f$. Coordinates in the moving frame and c.m. frame are related by standard Lorentz transformations. For example, if we consider a particle with mass $m$, momenta $\mathbf{k}$ and $\mathbf{k}^{*}$ in the moving and c.m. frames, then

$$
\sqrt{m^{2}+k^{* 2}}=\gamma\left(\sqrt{m^{2}+k^{2}}-\beta k_{\|}\right), \quad k_{\|}^{*}=\gamma\left(k_{\|}-\beta \sqrt{m^{2}+k^{2}}\right), \quad k_{\perp}^{*}=k_{\perp},
$$

where $\gamma=\frac{E}{E^{*}}$ and $\beta=\frac{|\mathbf{P}|}{E}$.

Two-particle interpolating operators in a given irrep can be written as a linear combination of products of single particle interpolating operators with appropriate Clebsch-Gordan coefficients $[226,186,219,220,221,156,222,223,225$. By first considering an energy range where only a single channel is present, one can readily write down the relevant two-body operator

$$
\mathcal{O}_{\Lambda \mu}\left(x_{0}, \mathbf{P},|\mathbf{P}-\mathbf{k}|,|\mathbf{k}|\right)=\sum_{R \in \mathrm{LG}(\mathbf{P})} \mathcal{C}(\mathbf{P} \Lambda \mu ; R \mathbf{k} ; R(\mathbf{P}-\mathbf{k})) \varphi\left(x_{0}, R \mathbf{k}\right) \widetilde{\varphi}\left(x_{0}, R(\mathbf{P}-\mathbf{k})\right)
$$

where in general $\varphi$ and $\widetilde{\varphi}$ may be identical or non-identical operators and $R$ is understood as an element of the representation of $\operatorname{LG}(\mathbf{P})$ defined by action on three-dimensional spatial vectors. In order to minimize unnecessary notation, we will suppress the dependence of $\mathcal{O}$ on $|\mathbf{P}-\mathbf{k}|$ and $|\mathbf{k}|$ from now on $9^{9}$

\footnotetext{
${ }^{9}$ All throughout this work $\mathcal{O}$ will denote an operator that has overlap with a two-particle state and $\varphi$ will refer to a single particle operator, although in general these two will access all states with the corresponding quantum numbers of the operator.
} 
To completely specify the Clebsch-Gordan coefficients, we now introduce $\{\mathbf{k}\}_{\mathbf{P}}$ as the set of all momenta that are reached by applying a rotation in $\operatorname{LG}(\mathbf{P})$ to $\mathbf{k}$. We then denote the irreps of particles one and two by $\Lambda_{1}\left(\{\mathbf{P}-\mathbf{k}\}_{\mathbf{P}}\right)$ and $\Lambda_{2}\left(\{\mathbf{k}\}_{\mathbf{P}}\right)$ respectively, and define the Clebsch-Gordan coefficient, $\mathcal{C}(\mathbf{P} \Lambda \mu ; R \mathbf{k} ; R(\mathbf{P}-\mathbf{k}))$, to project the two particles in $\Lambda_{1}\left(\{\mathbf{P}-\mathbf{k}\}_{\mathbf{P}}\right) \otimes \Lambda_{2}\left(\{\mathbf{k}\}_{\mathbf{P}}\right)$ onto $\Lambda(\mathbf{P}), \mu$. This may also be expressed as an innerproduct of states

$$
\mathcal{C}(\mathbf{P} \Lambda \mu ; R \mathbf{k} ; R(\mathbf{P}-\mathbf{k})) \equiv\left\langle\Lambda(\mathbf{P}), \mu \mid \Lambda_{1}\left(\{\mathbf{P}-\mathbf{k}\}_{\mathbf{P}}\right), R(\mathbf{P}-\mathbf{k}) ; \Lambda_{2}\left(\{\mathbf{k}\}_{\mathbf{P}}\right), R \mathbf{k}\right\rangle,
$$

from which follows

$$
\sum_{R \in \mathrm{LG}(\mathbf{P})}|\mathcal{C}(\mathbf{P} \Lambda \mu ; R \mathbf{k} ; R(\mathbf{P}-\mathbf{k}))|^{2}=1 .
$$

The simplest nontrivial example of this operator construction is reached by setting the total momentum to zero, setting $\mathbf{k}=\frac{2 \pi}{L} \hat{\mathbf{k}} \equiv q_{(1)} \hat{\mathbf{k}}$, and taking the two-particle operator to be in the $\mathbb{A}_{1}^{+}$irrep

$$
\begin{aligned}
\mathcal{O}_{\mathbb{A}_{1}^{+}}\left(x_{0}, \mathbf{0}\right)=\frac{\sigma}{\sqrt{6}}[ & \varphi\left(x_{0}, q_{(1)} \hat{\mathbf{z}}\right) \widetilde{\varphi}\left(x_{0},-q_{(1)} \hat{\mathbf{z}}\right)+\varphi\left(x_{0},-q_{(1)} \hat{\mathbf{z}}\right) \widetilde{\varphi}\left(x_{0}, q_{(1)} \hat{\mathbf{z}}\right) \\
& +\varphi\left(x_{0}, q_{(1)} \hat{\mathbf{x}}\right) \widetilde{\varphi}\left(x_{0},-q_{(1)} \hat{\mathbf{x}}\right)+\varphi\left(x_{0},-q_{(1)} \hat{\mathbf{x}}\right) \widetilde{\varphi}\left(x_{0}, q_{(1)} \hat{\mathbf{x}}\right) \\
& \left.+\varphi\left(x_{0}, q_{(1)} \hat{\mathbf{y}}\right) \widetilde{\varphi}\left(x_{0},-q_{(1)} \hat{\mathbf{y}}\right)+\varphi\left(x_{0},-q_{(1)} \hat{\mathbf{y}}\right) \widetilde{\varphi}\left(x_{0}, q_{(1)} \hat{\mathbf{y}}\right)\right],
\end{aligned}
$$

where $\sigma=\sqrt{1 / 2}$ if $\varphi$ and $\widetilde{\varphi}$ are the same operators and $\sigma=1$ otherwise. If we give the system a nonzero boost along $\hat{\mathbf{z}}$, then the symmetry group is reduced to $\mathrm{LG}(\hat{\mathbf{z}})$. Consider the scenario where the momentum of the $\varphi$ field has magnitude $q_{(1)}$ and that of $\widetilde{\varphi}$ has magnitude $\sqrt{2} q_{(1)}$. With these single-particle operators, we can construct a two-particle operator that transforms in the $\mathbb{A}_{1}$ irrep [186]

$$
\begin{aligned}
\mathcal{O}_{\mathbb{A}_{1}}\left(x_{0}, q_{(1)} \hat{\mathbf{z}}\right) & =\frac{1}{2}\left[\varphi\left(x_{0}, q_{(1)} \hat{\mathbf{x}}\right) \widetilde{\varphi}\left(x_{0}, q_{(1)}(\hat{\mathbf{z}}-\hat{\mathbf{x}})\right)+\varphi\left(x_{0}, q_{(1)} \hat{\mathbf{y}}\right) \widetilde{\varphi}\left(x_{0}, q_{(1)}(\hat{\mathbf{z}}-\hat{\mathbf{y}})\right)\right. \\
+ & \left.\varphi\left(x_{0},-q_{(1)} \hat{\mathbf{x}}\right) \widetilde{\varphi}\left(x_{0}, q_{(1)}(\hat{\mathbf{z}}+\hat{\mathbf{x}})\right)+\varphi\left(x_{0},-q_{(1)} \hat{\mathbf{y}}\right) \widetilde{\varphi}\left(x_{0}, q_{(1)}(\hat{\mathbf{z}}+\hat{\mathbf{y}})\right)\right]
\end{aligned}
$$

In general, there might be $N$ open channels contributing to a given state. For example, in the case where isospin is equal to zero or one, an infinite volume $\pi \pi$ state can mix with a $K \bar{K}$ state, and both must thus have nonzero overlap with the corresponding finite-volume 
state. It is convenient to introduce an index, e.g. " $a$ ", to the interpolating operator in Eq. 4.13 to indicate the infinite-volume channel that it interpolates

$$
\mathcal{O}_{\Lambda \mu}\left(x_{0}, \mathbf{P}\right) \longrightarrow \mathcal{O}_{\Lambda \mu, a}\left(x_{0}, \mathbf{P}\right)
$$

For example, $\mathcal{O}_{\Lambda \mu, a}$ could refer to a $\pi \pi$-like or a $K \bar{K}$-like operator. With this, we can write a generic correlation function for a two-particle system that has been projected onto a given irrep as

$$
\begin{array}{r}
C_{\Lambda \mu, a b}^{(2)}\left(x_{0}-y_{0}, \mathbf{P}\right)=\left\langle 0\left|\mathcal{O}_{\Lambda^{\prime} \mu^{\prime}, a}\left(x_{0}, \mathbf{P}\right) \mathcal{O}_{\Lambda \mu, b}^{\dagger}\left(y_{0},-\mathbf{P}\right)\right| 0\right\rangle \\
=\delta_{\Lambda, \Lambda^{\prime}} \delta_{\mu, \mu^{\prime}} \sum_{n} e^{-E_{\Lambda, n}\left(x_{0}-y_{0}\right)}\left\langle 0\left|\mathcal{O}_{\Lambda \mu, a}(0, \mathbf{P})\right| E_{\Lambda, n} \mathbf{P} ; L\right\rangle\left\langle E_{\Lambda, n} \mathbf{P} ; L\left|\mathcal{O}_{\Lambda \mu, b}^{\dagger}(0,-\mathbf{P})\right| 0\right\rangle \\
+\mathcal{O}\left(L^{6} e^{-E_{t h}\left(x_{0}-y_{0}\right)} / E_{t h}^{2}\right),
\end{array}
$$

where $E_{\Lambda, n}$ is the $n t h$ two-particle eigenenergy of the $\Lambda$-irrep of $\operatorname{LG}(\mathbf{P})$. This is the twobody analog of Eq. 4.5. In general we expect multiple two-body states below the first multi-particle threshold, $E_{t h}$, and hence include a sum over $n$.

The correlation function can also be written in terms of the interactions of the twoparticle system. The leading order (LO) contribution to the correlation function (first diagram in Fig. 4.1(a) is determined by considering the limit in which the interactions vanish, and as a result the different channels cannot mix. We find

$$
C_{\Lambda \mu, a b}^{(2, L O)}\left(x_{0}-y_{0}, \mathbf{P}\right)=L^{6} \int \frac{d P_{0}}{2 \pi} e^{i P_{0}\left(x_{0}-y_{0}\right)} \widetilde{C}_{\Lambda \mu, a b}^{(2, L O)}\left(P_{0}, \mathbf{P}\right),
$$

where

$$
\begin{aligned}
\widetilde{C}_{\Lambda \mu, a b}^{(2, L O)}\left(P_{0}, \mathbf{P}\right) \equiv & \delta_{a b} \frac{1}{\eta} \int \frac{d k_{0}}{2 \pi} \sum_{R \in L G(\mathbf{P})} \\
& \times \mathcal{C}(\mathbf{P} \Lambda \mu ; R \mathbf{k} ; R(\mathbf{P}-\mathbf{k})) G(k) G(P-k) \mathcal{C}^{*}(\mathbf{P} \Lambda \mu ; R \mathbf{k} ; R(\mathbf{P}-\mathbf{k}))
\end{aligned}
$$

Here we have introduced the fully dressed propagator

$$
G(k) \equiv \int d^{4} x e^{-i k x}\left\langle 0\left|T \phi(x) \phi^{\dagger}(0)\right| 0\right\rangle,
$$

with on-shell renormalization $\lim _{k_{0} \rightarrow i \omega_{k}}\left(k^{2}+m^{2}\right) G(k)=1$. We have also introduced the symmetry factor $\eta$ which is equal to $1 / 2$ if the particles identical and have momenta that are related by $\operatorname{LG}(\mathbf{P})$ rotations, and equal to 1 otherwise. 
Observe here that $G(k)$ is the infinite-volume fully dressed propagator. Really $\widetilde{C}_{\Lambda \mu, a b}^{(2, L O)}\left(P_{0}, \mathbf{P}\right)$ should be constructed from the finite-volume analog of $G(k)$. However, as long as $\left[P_{0}^{2}+\mathbf{P}^{2}\right]^{1 / 2}$ has an imaginary part with magnitude below $E_{t h}^{*}$, then using the infinitevolume propagator only incurs exponentially suppressed corrections of the form $e^{-m_{\pi} L}$, with $m_{\pi}$ the lightest mass in the spectrum. This is discussed in more detail in the context of the Bethe-Salpeter kernel below. We deduce that our expression for $\widetilde{C}_{\Lambda \mu, a b}^{(2, L O)}\left(P_{0}, \mathbf{P}\right)$ is only valid in a strip of the complex $P_{0}$ plane which runs along the real axis and is bounded by $\left[P_{0}^{2}+\mathbf{P}^{2}\right]=-E_{t h}^{* 2}$.

We now complete the analysis of $C_{\Lambda \mu, a b}^{(2, L O)}\left(x_{0}-y_{0}, \mathbf{P}\right)$, by first evaluating $k_{0}$ and $k_{0}^{\prime}$ integrals, and then evaluating the integral on $P_{0}$. Define

$$
\omega_{j, 1} \equiv \sqrt{m_{j, 1}^{2}+(\mathbf{P}-\mathbf{k})^{2}}, \quad \omega_{j, 2} \equiv \sqrt{m_{j, 2}^{2}+\mathbf{k}^{2}} .
$$

In performing the $k_{0}$ and $k_{0}^{\prime}$ integrals we encircle the pole at $i \omega_{j, 2}$ and this fixes the "2" particle in the $j t h$ channel to be on-shell with free energy $\omega_{j, 2}$. By energy conservation, the "1" particle will have energy $-i P_{0}-\omega_{j, 2}$. Specifically we find

$$
\begin{array}{r}
C_{\Lambda \mu, a b}^{(2, L O)}\left(x_{0}-y_{0}, \mathbf{P}\right)=\delta_{a b} \frac{L^{6}}{\eta} \int \frac{d P_{0}}{2 \pi} e^{i P_{0}\left(x_{0}-y_{0}\right)} \sum_{R \in L G(\mathbf{P})} \frac{|\mathcal{C}(\mathbf{P} \Lambda \mu ; R \mathbf{k} ; R(\mathbf{P}-\mathbf{k}))|^{2}}{4 \omega_{a, 1} \omega_{a, 2}\left(i P_{0}+\left(\omega_{a, 1}+\omega_{a, 2}\right)\right)} \\
+\mathcal{O}\left(L^{6} e^{-E_{t h}\left(x_{0}-y_{0}\right)} / E_{t h}^{2}\right)
\end{array}
$$

Note here that the first term gives a pole in the $P_{0}$ plane that sits in the region where our expression for $\widetilde{C}_{\Lambda \mu, a b}^{(2, L O)}\left(P_{0}, \mathbf{P}\right)$ is valid. We do not control the exact form of the second term, which decays according to some above-threshold energy. The precise form of the above threshold term is not needed for our final result.

To include higher orders we need only assume that the correlation function, defined in Eq. 4.19, is correctly reproduced by the all-orders summation of a skeleton expansion built from Bethe-Salpeter kernels and fully dressed propagators. In particular we define the NLO correlator as the contribution built from a single insertion of the Bethe-Salpeter kernel, $K$. The kernel is depicted in Fig. 4.1(b) and is defined as the sum of all amputated four-point diagrams that are two-particle irreducible in the s-channel. We find

$$
C_{\Lambda \mu, a b}^{(2, N L O)}\left(x_{0}-y_{0}, \mathbf{P}\right)=L^{6} \int \frac{d P_{0}}{2 \pi} e^{i P_{0}\left(x_{0}-y_{0}\right)} \widetilde{C}_{\Lambda \mu, a b}^{(2, N L O)}\left(P_{0}, \mathbf{P}\right),
$$




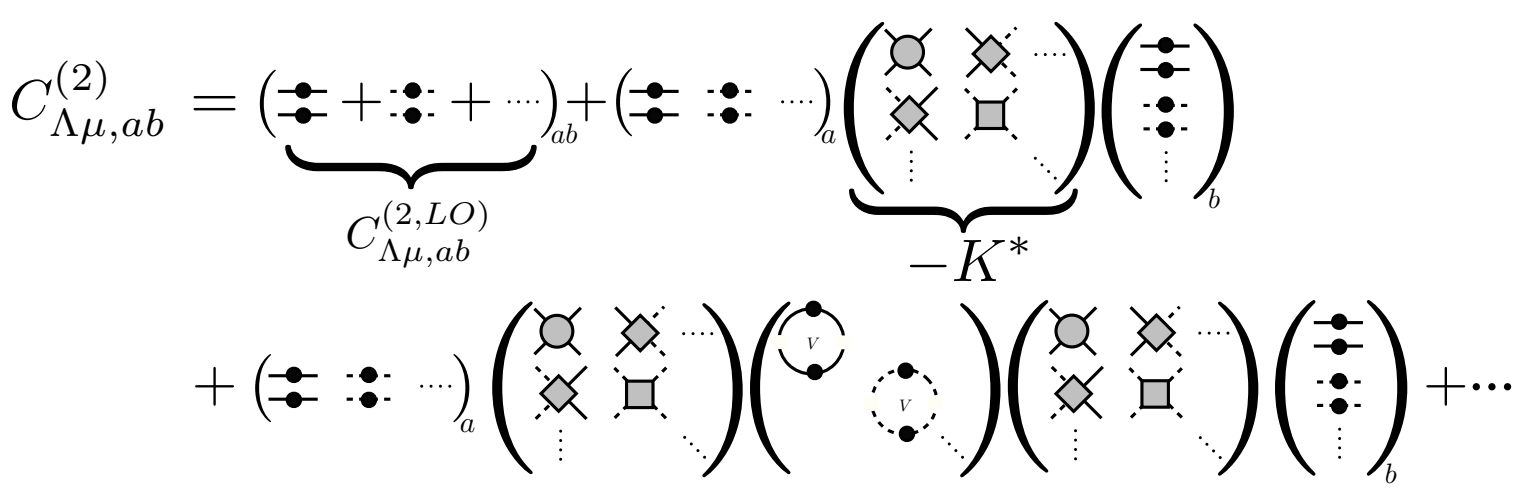

(a)

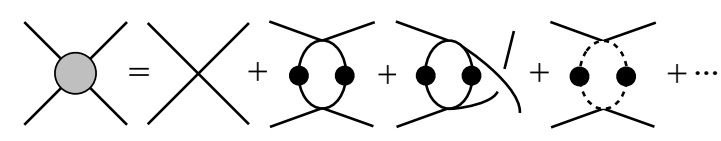

(b)

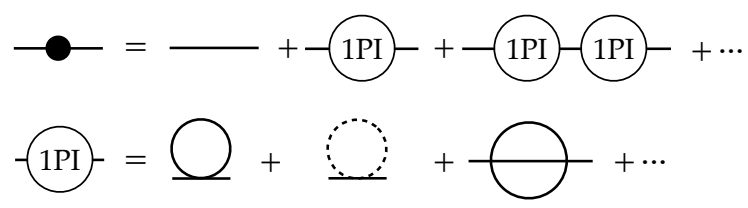

(c)

Figure 4.1: a) Shown is the definition of the finite volume two-particle correlation function. The solid lines denote two-particles in the "1" channel, dashed lines denote particle in the "2" channel. The correlation function is written in terms of the c.m. kernel, $K^{*}$, and the fully dressed single particle propagators. b) Shown is $K^{*}$ for the first channel, which is the sum of all two-particle irreducible s-channel diagrams. Explicitly shown are examples of diagrams that are included in the kernel: contact interactions, $\mathrm{t}$ - and u-channel diagrams. In general, all diagrams allowed by the underlying theory where the intermediate particles cannot all simultaneously go on-shell are absorbed into the kernel. As described in the text, in this study we are restricted to energies where only two-particle states are allowed to go on-shell. c) Shown is the definition of the fully dressed one particle propagator in terms of the one particle irreducible (1PI) diagrams. 
where

$$
\begin{aligned}
\widetilde{C}_{\Lambda \mu, a b}^{(2, N L O)}\left(P_{0}, \mathbf{P}\right)=-\frac{1}{L^{3}} \sum_{R, R^{\prime} \in \mathrm{LG}(\mathbf{P})} \mathcal{C}\left(\mathbf{P} \Lambda \mu ; R^{\prime} \mathbf{k} ; R^{\prime}(\mathbf{P}-\mathbf{k})\right) \\
\quad \times \int \frac{d k_{0}^{\prime}}{2 \pi} \int \frac{d k_{0}}{2 \pi} G\left(k^{\prime}\right) G\left(P-k^{\prime}\right) K\left(P, k, k^{\prime}\right) G(k) G(P-k) \mathcal{C}^{*}(\mathbf{P} \Lambda \mu ; R \mathbf{k} ; R(\mathbf{P}-\mathbf{k})) .
\end{aligned}
$$

In general, the kernel is a function of volume, but since the c.m. energy is restricted to satisfy $m_{1}+m_{2} \leq E^{*} \ll E_{t h}^{*}$ the intermediate particles appearing in the kernel cannot all simultaneously go on-shell. Therefore, one can show using Poisson's resummation formula

$$
\left[\frac{1}{L^{3}} \sum_{\mathbf{q}}\right] f(\mathbf{q})=\sum_{\mathbf{n} \neq 0} \int \frac{d \mathbf{q}}{(2 \pi)^{3}} f(\mathbf{q}) e^{i L \mathbf{n} \cdot \mathbf{q}},
$$

that the difference between finite- and infinite-volume kernels is exponentially small in $m L$. In writing the Poisson resummation formula the following notation has been introduced

$$
\left[\frac{1}{L^{3}} \sum_{\mathbf{q}}\right] \equiv\left(\frac{1}{L^{3}} \sum_{\mathbf{q}}-\int \frac{d \mathbf{q}}{(2 \pi)^{3}}\right) .
$$

Since we neglect these corrections, the result discussed here holds only for $m_{\pi} L \gg 1$. We will neglect any terms in the correlation function that are exponentially suppressed with the mass of any particle in any coupled channel since $\mathcal{O}\left(e^{-m_{i} L}\right) \leq \mathcal{O}\left(e^{-m_{\pi} L}\right)$. These corrections have been previously determine for $\pi \pi$ [229] and $N N$ systems [230] in an S-wave, as well as the $\pi \pi$ system in a P-wave in Ref. [231, 232].

Higher order contributions to the correlation function can be readily evaluated by making the following replacement

$$
-\left[K\left(P, k, k^{\prime}\right)\right]_{a, b} \longrightarrow-\left[\mathbb{T}_{L}\left(P, k, k^{\prime}\right)\right]_{a, b},
$$

where

$$
\begin{aligned}
- & {\left[\mathbb{T}_{L}\left(P, k, k^{\prime}\right)\right]_{a, b} } \\
& =-\left[K\left(P, k, k^{\prime}\right)\right]_{a, b}+\int \frac{d l_{0}}{2 \pi} \frac{\xi_{j}}{L^{3}} \sum_{\mathbf{l}}[K(P, k, l)]_{a, j} G_{j}(l) G_{j}(P-l)\left[\mathbb{T}_{L}\left(P, l, k^{\prime}\right)\right]_{j, b},
\end{aligned}
$$

and the summation over the intermediate channel $j$ is implicit. 
A convenient expression for $\mathbb{T}_{L}$ can be found utilizing the machinery developed by Kim, Sachrajda, and Sharpe [130]. In order to determine the finite-volume corrections to the correlation function, it is sufficient to know the difference between finite-volume momentum sum and infinite-volume momentum integral acting on the two-particle poles. Using a principal-value prescription to define the integral at the pole, we observe

$$
\begin{array}{r}
\xi_{j}\left[\frac{1}{L^{3}} y_{\mathbf{1}}\right] \mathrm{P} . \mathrm{V} \cdot \frac{[K(P, k, l)]_{a, j}\left[K\left(P, l, k^{\prime}\right)\right]_{j, b}}{4 \omega_{1, \mathbf{P}-\mathbf{1}} \omega_{2, \mathbf{l}}\left(\omega_{1, \mathbf{P}-\mathbf{1}}+\omega_{2, \mathbf{1}}-P_{0, M}\right)} \equiv \\
-\left[K_{o f f, o n}^{*} \mathbb{F}^{V} K_{o n, o f f}^{*}\right]_{a, b}+\mathcal{O}\left(e^{-m_{\pi} L}\right),
\end{array}
$$

where the c.m. kernel, $K_{o f f, o n}^{*}$, is the kernel for a system where the two incoming particles are evaluated on-shell, while the outgoing particles are left off-shell. Here we have also introduced the Minkowski energy $P_{0, M} \equiv-i P_{0}$. Note, if one chooses to use an $i \epsilon$ prescription for the propagator, this would lead to a second contribution to the right-hand side of Eq. 4.30, due to the residue of the infinite volume integral on the left hand side. This choice does not affect our result for the finite-volume correlation function.

In writing the right-hand side of Eq. 4.30, the kernels and the finite volume function have been written as matrices over angular momentum. The matrix elements of $\mathbb{F}^{V}$ in the spherical harmonic basis are found to be [130, 140, 146]

$$
\left[\mathbb{F}_{j}^{V}\right]_{l m_{l} ; l^{\prime} m_{l^{\prime}}}=-\frac{\xi_{j}}{8 \pi P_{0, M}^{*}}\left[\sum_{l^{\prime \prime}, m^{\prime \prime}} \frac{(4 \pi)^{3 / 2}}{k_{j, \text { on }}^{* l^{\prime \prime}}} c_{l^{\prime \prime} m^{\prime \prime}}^{\mathbf{d}}\left(k_{j, o n}^{* 2} ; L\right) \int d \Omega Y_{l, m_{l}}^{*} Y_{l^{\prime \prime}, m^{\prime \prime}}^{*} Y_{l^{\prime}, m_{l^{\prime}}}\right] .
$$

The function $c_{l m}^{\mathbf{d}}$ is defined as

$$
c_{l m}^{\mathbf{d}}\left(k_{j}^{* 2} ; L\right)=\frac{\sqrt{4 \pi}}{\gamma L^{3}}\left(\frac{2 \pi}{L}\right)^{l-2} \mathcal{Z}_{l m}^{\mathbf{d}}\left[1 ;\left(k_{j}^{*} L / 2 \pi\right)^{2}\right], \quad \mathcal{Z}_{l m}^{\mathbf{d}}\left[s ; x^{2}\right]=\sum_{\mathbf{r} \in \mathcal{P}_{\mathbf{d}}} \frac{|\mathbf{r}|^{l} Y_{l, m}(\mathbf{r})}{\left(r^{2}-x^{2}\right)^{s}},
$$

where $\gamma=P_{0, M} / P_{0, M}^{*}$, the sum is performed over $\mathcal{P}_{\mathbf{d}}=\left\{\mathbf{r} \in \mathbf{R}^{3} \mid \mathbf{r}=\hat{\gamma}^{-1}\left(\mathbf{m}-\alpha_{j} \mathbf{d}\right)\right\}, \mathbf{m}$ is a triplet integer, $\mathbf{d}$ is the normalized boost vector $\mathbf{d}=\mathbf{P} L / 2 \pi, \alpha_{j}=\frac{1}{2}\left[1+\left(m_{j, 1}^{2}-m_{j, 2}^{2}\right) / P_{0, M}^{* 2}\right]$ [143, 144, 145], and $\hat{\gamma}^{-1} \mathbf{x} \equiv \gamma^{-1} \mathbf{x}_{\|}+\mathbf{x}_{\perp}$, with $\mathbf{x}_{\|}\left(\mathbf{x}_{\perp}\right)$ denoting the $\mathbf{x}$ component that is parallel(perpendicular) to the total momentum, $\mathbf{P}$. In Appendix $\mathrm{C}$ we show the generalization of this for asymmetric volumes with twisted boundary conditions.

We mention a subtlety here with the definition of $c_{l m}^{\mathbf{d}}\left(k_{j}^{* 2} ; L\right)$ for $k_{j}^{* 2}<0$. The definitions given above continue to hold for subthreshold momenta, but only if the appropriate analytic 
continuation is implemented. To understand this in detail we first observe that the sum defining $\mathcal{Z}_{l m}^{\mathrm{d}}$ diverges for $s<3 / 2+l / 2$ and in particular diverges for $s=1$. The function $\mathcal{Z}_{l m}^{\mathbf{d}}$ is thus understood to be defined via analytic continuation from $s>3 / 2+l / 2$. To make this definition more apparent in the present context we give the equivalent form from Kim, Sachrajda and Sharpe

$$
\begin{aligned}
c_{l m}^{\mathbf{d}}\left(k_{j, o n}^{* 2} ; L\right)=-\frac{1}{\gamma L^{3}} \sum_{\mathbf{k}^{*}} \frac{\exp \left[\alpha\left(k_{j, o n}^{* 2}-k^{* 2}\right)\right]}{k_{j, o n}^{* 2}-k^{* 2}} & k^{* l} \sqrt{4 \pi} Y_{l m}\left(\hat{\mathbf{k}}^{*}\right) \\
& +\delta_{l 0} \text { P.V. } \int \frac{d \mathbf{k}^{*}}{(2 \pi)^{3}} \frac{\exp \left[\alpha\left(k_{j, o n}^{* 2}-k^{* 2}\right)\right]}{k_{j, \text { on }}^{* 2}-k^{* 2}},
\end{aligned}
$$

where the sum is over all $\mathbf{k}^{*} \in(2 \pi / L) \mathcal{P}_{\mathbf{d}}$ and the limit $\alpha \rightarrow 0^{+}$is understood. This definition makes the ultraviolet regularization, which is implicit in the analytic continuation in $s$, more explicit. For continuation to $k_{j, o n}^{* 2}<0$ it is convenient to rewrite the integral as an $i \epsilon$ prescription and a remainder

$$
\text { P.V. } \int \frac{d \mathbf{k}^{*}}{(2 \pi)^{3}} \frac{\exp \left[\alpha\left(k_{j, \text { on }}^{* 2}-k^{* 2}\right)\right]}{k_{j, \text { on }}^{* 2}-k^{* 2}}=\int \frac{d \mathbf{k}^{*}}{(2 \pi)^{3}} \frac{\exp \left[\alpha\left(k_{j, \text { on }}^{* 2}-k^{* 2}\right)\right]}{k_{j, \text { on }}^{* 2}-k^{* 2}+i \epsilon}+\frac{i k_{j, \text { on }}^{*}}{4 \pi} .
$$

The subthreshold continuation of the left hand ride is defined as the following limit of the right-hand side

$$
\begin{aligned}
\lim _{k_{j, \text { on }}^{*} \rightarrow i \kappa_{j}}\left[\int \frac{d \mathbf{k}^{*}}{(2 \pi)^{3}} \frac{\exp \left[\alpha\left(k_{j, \text { on }}^{* 2}-k^{* 2}\right)\right]}{k_{j, \text { on }}^{* 2}-k^{* 2}+i \epsilon}\right. & \left.+\frac{i k_{j, \text { on }}^{*}}{4 \pi}\right] \\
& =-\left[\int \frac{d \mathbf{k}^{*}}{(2 \pi)^{3}} \frac{\exp \left[-\alpha\left(\kappa_{j}^{2}+k^{* 2}\right)\right]}{\kappa_{j}^{2}+k^{* 2}}+\frac{\kappa_{j}}{4 \pi}\right],
\end{aligned}
$$

where $\kappa_{j}$ is the binding momentum of the $j$ th channel.

We next turn to the Bethe-Salpeter kernel which, like $\mathbb{F}^{V}$, can be expressed as a matrix in angular momentum

$$
K_{o f f, o f f}^{*}\left(P_{0}^{*}, \mathbf{k}_{i}^{*}, \mathbf{k}_{f}^{*}\right)=4 \pi \sum_{l, m_{l}, l^{\prime}, m_{l^{\prime}}} Y_{l m_{l}}\left(\hat{\mathbf{k}}_{f}^{*}\right) Y_{l^{\prime} m_{l^{\prime}}}^{*}\left(\hat{\mathbf{k}}_{i}^{*}\right)\left[K_{o f f, o f f}^{*}\left(P_{0}^{*}, k_{i}^{*}, k_{j}^{*}\right)\right]_{l m_{l}, l^{\prime} m_{l^{\prime}}} .
$$

Here we consider a kernel in which both the initial and final states are off-shell. More precisely, we assume $k_{i, 0}=i \omega_{\mathbf{k}_{i}}$ and $k_{f, 0}=i \omega_{\mathbf{k}_{f}}$, but no additional constraints. These

${ }^{10}$ Our definition of $c_{l m}$ differs from Ref. [130] by an overall sign. 
relations, which arise from contour integration as discussed, do not give on-shell two-particle states since $P_{0}-k_{0, i} \neq i \omega_{\mathbf{P}-\mathbf{k}_{i}}$ and $P_{0}-k_{0, f} \neq i \omega_{\mathbf{P}-\mathbf{k}_{f}}$. Nevertheless, it is still possible to change to the c.m. frame, expressing the kernel in terms of $\left(P_{0}^{*}, \mathbf{k}_{i}^{*}, \mathbf{k}_{f}^{*}\right)$ as indicated above. Note that the matrix defined in Eq. 4.36 is diagonal,

$$
\left[K_{o f f, o f f}^{*}\left(P_{0}^{*}, k_{i}^{*}, k_{j}^{*}\right)\right]_{l m_{l}, l^{\prime} m_{l^{\prime}}} \propto \delta_{l, l^{\prime}} \delta_{m_{l}, m_{l}^{\prime}}
$$

This follows from the rotational invariance of the infinite-volume theory, equivalently from the fact that the only angular dependence in the c.m. frame is $\hat{\mathbf{k}}_{i}^{*} \cdot \hat{\mathbf{k}}_{f}^{*}$. Finally, we comment that the on-shell point is contained within Eq. 4.36 and is accessed by constraining the three momenta magnitudes to $k_{i}^{*}=k_{f}^{*}=k_{o n}^{*}$. We return to this discussion in the context of the quantization condition below.

Directly following Kim, Sachrajda and Sharpe by summing over all possible insertions of the Bethe-Salpeter kernel, we find

$$
-\mathbb{T}_{L}=\mathbb{K}_{\text {off,off }}-\mathbb{K}_{o f f, o n} \frac{1}{1+\mathbb{F}^{V} \mathbb{K}} \mathbb{F}^{V} \mathbb{K}_{o n, o f f} .
$$

Here we have introduced the two-to-two K-matrix, which is defined as the sum of all infinitevolume, amputated $\mathbf{2} \rightarrow \mathbf{2}$ diagrams with loop integrals defined via principal-value prescription 11

$$
\begin{aligned}
{\left[\mathbb{K}\left(P, k, k^{\prime}\right)\right]_{a, b} \equiv } & -\left[K\left(P, k, k^{\prime}\right)\right]_{a, b} \\
& +\xi_{j} \text { P.V. } \int \frac{d \mathbf{l}}{(2 \pi)^{3}} \int \frac{d l_{0}}{2 \pi}[K(P, k, l)]_{a, j} G_{j}(l) G_{j}(P-l)\left[\mathbb{K}\left(P, l, k^{\prime}\right)\right]_{j, b} .
\end{aligned}
$$

This object is explicitly shown in Fig. 4.2(b) for a single channel scenario. Observe that in Eq. 4.38 we have given subscripts on $\mathbb{K}$ to indicate whether the incoming and outgoing states are on or off-shell. $\mathbb{K}$ with no subscript is reserved for the on-shell K-matrix.

\footnotetext{
${ }^{11}$ The use of pole prescription here is somewhat subtle. If we restrict the Euclidean-signature time component $P_{0}$ to be real, then no pole prescription is needed. However if $P_{0}$ is imaginary and thus $P_{0, M}$ is real, then poles appear in the region of integration. Our definition requires always performing time component integrals with $P_{0, M}$ off the real axis, as in the standard $i \epsilon$ prescription. This produces integrals over spatial components of the form of Eq. 4.30. These are always to be evaluated with real $P_{0, M}$ and with the principal value pole prescription. Alternatively one may use the $i \epsilon$ prescription for the entirety of each two-particle loop integral, but then one must take only the real part.
} 
We contrast the K-matrix to the scattering amplitude, $\mathcal{M}$, which is defined as the sum of all infinite-volume, amputated $\mathbf{2} \rightarrow \mathbf{2}$ diagrams with integration defined via $i \epsilon$ prescription (as shown in Fig. 4.2(a) for a single channel)

$$
\begin{aligned}
{\left[\mathcal{M}\left(P, k, k^{\prime}\right)\right]_{a, b} \equiv-[K(P,} & \left.\left.k, k^{\prime}\right)\right]_{a, b} \\
& +\xi_{j} \int \frac{d^{4} l}{(2 \pi)^{4}}[K(P, k, l)]_{a, j} G_{j}(l) G_{j}(P-l)\left[\mathcal{M}\left(P, l, k^{\prime}\right)\right]_{j, b} .
\end{aligned}
$$

The on-shell K-matrix can be directly related to the on-shell scattering amplitude by introducing a kinematic matrix that is diagonal over the $\mathrm{N}$ open channels $\mathbb{P}=\operatorname{diag}\left(\sqrt{\xi_{1} q_{1}^{*}}, \sqrt{\xi_{1} q_{1}^{*}}, \ldots, \sqrt{\xi_{N} q_{N}^{*}}\right) / \sqrt{4 \pi E^{*}}$. For a system with angular momentum $J=l=l^{\prime}$, the amplitudes $\mathcal{M}_{J}$ and $\mathbb{K}_{J}$ are related via [140],

$$
\mathcal{M}_{J}^{-1}=\mathbb{K}_{J}^{-1}-i \mathbb{P}^{2} / 2
$$

and the scattering amplitude and the $S$-matrix via

$$
i \mathcal{M}_{J}=\mathbb{P}^{-1}\left(S_{J}-\mathbb{I}\right) \mathbb{P}^{-1}
$$

Substituting $\mathbb{T}_{L}$ for $K$ in Eq. 4.26 gives the full correlation function

$$
\begin{aligned}
& C_{\Lambda \mu, a b}^{(2)}\left(x_{0}-y_{0}, \mathbf{P}\right)= \\
& L^{6} \int \frac{d P_{0}}{2 \pi} e^{i P_{0}\left(x_{0}-y_{0}\right)}\left\{\widetilde{C}_{\Lambda \mu, a b}^{[2, \mathcal{O}(\mathbb{K})]}\left(P_{0}, \mathbf{P}\right)-\mathbb{C}_{\Lambda \mu}^{T}\left[\frac{\mathbb{Y}}{L^{3}} \frac{1}{\mathbb{K}+\left(\mathbb{F}^{V}\right)^{-1}} \mathbb{Y}^{\dagger}\right]_{a b} \mathbb{C}_{\Lambda \mu}^{*}\right\} \\
& +\mathcal{O}\left(L^{6} e^{-E_{t h}\left(x_{0}-y_{0}\right)} / E_{t h}^{2}\right) .
\end{aligned}
$$

The first term of the integrand is defined as

$$
\begin{array}{r}
\widetilde{C}_{\Lambda \mu, a b}^{[2, \mathcal{O}(\mathbb{K})]}\left(P_{0}, \mathbf{P}\right) \equiv \widetilde{C}_{\Lambda \mu, a b}^{(2, L O)}\left(P_{0}, \mathbf{P}\right)+\frac{1}{L^{3}} \sum_{R, R^{\prime} \in \mathrm{LG}(\mathbf{P})} \mathcal{C}\left(\mathbf{P} \Lambda \mu ; R^{\prime} \mathbf{k} ; R^{\prime}(\mathbf{P}-\mathbf{k})\right) \\
\times \int \frac{d k_{0}^{\prime}}{2 \pi} \int \frac{d k_{0}}{2 \pi} G\left(k^{\prime}\right) G\left(P-k^{\prime}\right) \mathbb{K}_{o f f, o f f}\left(P, k, k^{\prime}\right) G(k) G(P-k) \\
\times \mathcal{C}^{*}(\mathbf{P} \Lambda \mu ; R \mathbf{k} ; R(\mathbf{P}-\mathbf{k})) .
\end{array}
$$




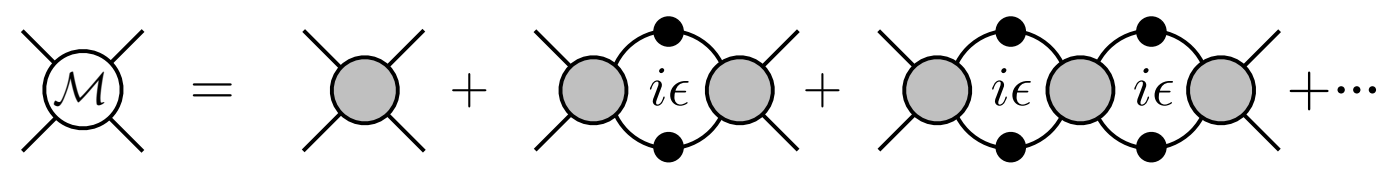

(a)

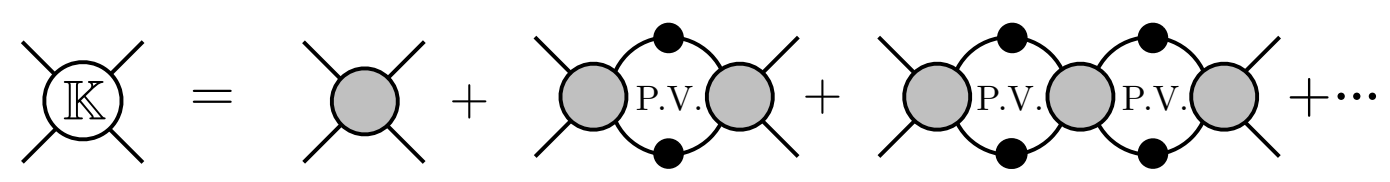

(b)

Figure 4.2: In order to illustrate the differences and similarities between the a) scattering amplitude, $\mathcal{M}$, and the b) K-matrix, $\mathbb{K}$, we show their diagrammatically representation for the single-channel case in terms of the kernels (defined in Fig. 4.1(b) and infinite volume loops. The infinite volume loops of the scattering amplitude are evaluated using the $i \epsilon$ prescription, while those of the K-matrix are evaluated using the principal value, as explicitly shown. For multichannel scenarios one simply upgrades the kernels and two-particle loops to be matrices in the number of open channels as depicted in Fig. 4.1. Note that the single particle propagators are fully dressed as defined in Fig. 4.1(c). 
We have also introduced new notation for the second term

$$
\begin{aligned}
{\left[\mathbb{C}_{\Lambda \mu}\right]_{l m, a} } & \equiv \sqrt{4 \pi} \sum_{R \in \mathrm{LG}(\mathbf{P})} \mathcal{C}(\mathbf{P} \Lambda \mu ; R(\mathbf{P}-\mathbf{k}) ; R \mathbf{k}) Y_{l m}\left(\widehat{R \mathbf{k}}^{*}\right), \\
{\left[\mathbb{Y}\left(P_{0}, R(\mathbf{P}-\mathbf{k}), R \mathbf{k}, k\right)\right]_{a, b} } & \equiv \int \frac{d k_{0}^{\prime}}{2 \pi} G\left(k_{0}^{\prime}, R \mathbf{k}\right) G\left(P_{0}-k_{0}^{\prime}, R(\mathbf{P}-\mathbf{k})\right) \mathbb{K}_{o f f, o n}\left(P, k, k^{\prime}\right)_{a, b}, \\
& \equiv 4 \pi Y_{l^{\prime} m^{\prime}}\left(\widehat{R \mathbf{k}}^{*}\right)\left[\mathbb{Y}\left(P_{0}^{*}\right)\right]_{a, l^{\prime}, m^{\prime} ; b, l, m} Y_{l, m}^{*}\left(\hat{k}^{*}\right) .
\end{aligned}
$$

We stress that $\mathbb{Y}$ depends on off-shell K-matrices. This dependence is unavoidable in the two-particle correlation function and will persist in our final result. However, we will see that the off-shell contributions cancel when we consider the ratio of finite and infinite-volume matrix elements of external currents.

In order to evaluate the integral over $P_{0}$ we first note that the free poles of the integrand exactly cancel. This is a nontrivial observation that cannot be reached unless one formally keeps all partial wave contributions that have overlap with the irrep of interest. In particular, in Appendix B, along with showing an explicit proof of the cancelation of the free poles, we show that by truncating the scattering amplitude to be in an S-wave the free poles in general do not cancel. The cancellation of free poles assures that the only contribution to Eq. 4.43 is from integration around poles of the interacting system. To evaluate these, we introduce

$$
\mathbb{M}\left(P_{0, M}\right) \equiv \mathbb{K}\left(P_{0, M}\right)+\left(\mathbb{F}^{V}\left(P_{0, M}\right)\right)^{-1}
$$

Now note that the finite-volume two-particle spectrum is given by energies for which $\mathbb{M}\left(P_{0, M}\right)$ has a vanishing eigenvalue. This is Lüscher's quantization condition, given in Eq. 4.1 above. At this stage we think it useful to discuss the connection of this result to previous work. We first observe that, although the condition in terms of $\mathbb{M}\left(P_{0, M}\right)$ is most convenient for the bulk of our analysis, here it is useful to reexpress it as

$$
\operatorname{det}\left[\left(\mathbb{K}\left(P_{0, M}\right)\right)^{-1}+\mathbb{F}^{V}\left(P_{0, M}\right)\right]=0
$$

Substituting Eq. 4.41 into this form then gives

$$
\operatorname{det}\left[\left(\mathcal{M}\left(P_{0, M}\right)\right)^{-1}+\mathbb{F}_{i \epsilon}^{V}\left(P_{0, M}\right)\right]=0
$$


where $\mathbb{F}_{i \epsilon}^{V}\left(P_{0, M}\right) \equiv i \mathbb{P}^{2} / 2+\mathbb{F}^{V}\left(P_{0, M}\right)$. This shows the equivalence of the present result to those appearing in Refs. [127, 128, 129, 130, 140, 146].

Next we consider Eq. 4.48 for energies in the vicinity of the lowest two-particle threshold. In this case we need only consider the S-wave scattering for the lowest two-particle channel. The quantization condition becomes

$$
\frac{\xi}{8 \pi P_{0, M}^{*}}\left[k^{*} \cot \delta\left(k^{*}\right)-4 \pi c_{00}^{\mathbf{d}}\left(k^{* 2} ; L\right)\right]=0 .
$$

We may analytically continue this result below threshold by replacing $k^{*}=i\left|k^{*}\right|=i \kappa$. In this continuation $4 \pi c_{00}^{\mathbf{d}}\left(k^{* 2} ; L\right)=-\kappa$ plus exponentially suppressed corrections 12 We deduce

$$
\left.k^{*} \cot \delta\left(k^{*}\right)\right|_{k^{*}=i \kappa}+\kappa=0,
$$

which is the standard, infinite-volume condition for a bound state.

Returning to the $P_{0}$ integral in Eq. 4.43 , we write the inverse of $\mathbb{M}\left(P_{0, M}\right)$ in terms of a determinant and adjugate,

$$
\frac{1}{\mathbb{M}\left(P_{0, M}\right)} \equiv \frac{1}{\operatorname{det}\left[\mathbb{M}\left(P_{0, M}\right)\right]} \operatorname{adj}\left[\mathbb{M}\left(P_{0, M}\right)\right]
$$

This equation defines the adjugate which is also equal to the transpose of the cofactor matrix. It implies that, as $P_{0, M}$ approaches a two-particle energy, $\mathbb{M}\left(P_{0, M}\right)^{-1}$ will diverge in proportion to $\operatorname{det}\left[\mathbb{M}\left(P_{0, M}\right)\right]^{-1}$ such that $\operatorname{adj}\left[\mathbb{M}\left(P_{0, M}\right)\right]$ remains finite. This separation, into diverging prefactor times finite matrix, makes Eq. 4.52 useful for evaluating the residue of the two-particle poles. Looking at the variation of the quantization condition about the energy eigenvalues, we find

$$
\begin{aligned}
\operatorname{det}\left[\mathbb{M}\left(P_{0, M}\right)\right] \mid= & \operatorname{det}\left[\mathbb{M}\left(E_{n}\right)\right] \\
& +\left.\left(P_{0, M}-E_{n}\right) \operatorname{tr}\left[\operatorname{adj}\left[\mathbb{M}\left(P_{0, M}\right)\right] \frac{\partial \mathbb{M}\left(P_{0, M}\right)}{\partial P_{0, M}}\right]\right|_{P_{0, M}=E_{n}}+\mathcal{O}\left(\left(P_{0}-i E_{n}\right)^{2}\right) \\
= & -\left.i\left(P_{0}-i E_{n}\right) \operatorname{tr}\left[\operatorname{adj}\left[\mathbb{M}\left(P_{0, M}\right)\right] \frac{\partial \mathbb{M}\left(P_{0, M}\right)}{\partial P_{0, M}}\right]\right|_{P_{0, M}=E_{n}}+\mathcal{O}\left(\left(P_{0}-i E_{n}\right)^{2}\right) .
\end{aligned}
$$

\footnotetext{
${ }^{12}$ We stress that this corrections may be large near threshold so that keeping the exponentially suppressed terms may be important.
} 
With this in hand, one can perform the integral in Eq. 4.43 to find

$$
\begin{aligned}
C_{\Lambda \mu, a b}^{(2)}\left(x_{0}-y_{0}, \mathbf{P}\right) & =L^{3} \sum_{n} e^{-E_{\Lambda, n}\left(x_{0}-y_{0}\right)} \mathbb{C}_{\Lambda \mu}^{T}\left[\mathbb{Y}_{\Lambda, n} R_{\Lambda, n} \mathbb{Y}_{\Lambda, n}^{\dagger}\right]_{a b} \mathbb{C}_{\Lambda \mu}^{*} \\
R_{\Lambda, n} & =\left.\operatorname{adj}\left[\mathbb{M}\left(P_{0, M}\right)\right] \operatorname{tr}\left[\operatorname{adj}\left[\mathbb{M}\left(P_{0, M}\right)\right] \frac{\partial \mathbb{M}\left(P_{0, M}\right)}{\partial P_{0, M}}\right]^{-1}\right|_{P_{0, M}=E_{\Lambda, n}}
\end{aligned}
$$

where $\mathbb{Y}_{\Lambda, n}$ is the value of $\mathbb{Y}$ (defined in Eq. 4.46) if evaluated at the $n t h$ interactive twoparticle pole. Here the sum over $n$ runs over a finite set of energies that lie below the next multi-particle threshold. We are constrained to this region because our expression for the integrand of the $P_{0}$ integral was only valid for a range of imaginary $P_{0}$ as already discussed above.

By comparing this result to Eq.4.19, we find that the matrix elements of the interpolating operators in general satisfy

$$
\begin{aligned}
& \left\langle 0\left|\mathcal{O}_{\Lambda \mu, a}(0, \mathbf{P})\right| E_{\Lambda, n} \mathbf{P} ; L\right\rangle\left\langle E_{\Lambda, n} \mathbf{P} ; L\left|\mathcal{O}_{\Lambda \mu, b}(0, \mathbf{P})\right| 0\right\rangle= \\
& L^{3} \mathbb{C}_{\Lambda \mu}^{T}\left[\mathbb{Y}_{\Lambda, n} R_{\Lambda, n} \mathbb{Y}_{\Lambda, n}^{\dagger}\right]_{a b} \mathbb{C}_{\Lambda \mu}^{*}
\end{aligned}
$$

and in the case that $a=b$ it implies

$$
\left|\left\langle 0\left|\mathcal{O}_{\Lambda \mu, a}(0, \mathbf{P})\right| E_{\Lambda, n} \mathbf{P} ; L\right\rangle\right|=L^{3 / 2} \sqrt{\mathbb{C}_{\Lambda \mu}^{T}\left[\mathbb{Y}_{\Lambda, n} R_{\Lambda, n} \mathbb{Y}_{\Lambda, n}^{\dagger}\right]_{a a} \mathbb{C}_{\Lambda \mu}^{*}},
$$

where the repeated indices in the right-hand side are not summed. Equations $4.57 \& 4.58$ are the main results of this section.

We now turn to applying this result to specific examples. In doing so we find it useful to introduce

$$
\begin{aligned}
\mathcal{N}_{\Lambda}^{\star} \equiv & \mathbb{C}_{\Lambda \mu}^{T} \mathbb{C}_{\Lambda \mu}^{*}=4 \pi \sum_{R, R^{\prime} \in \mathrm{LG}(\mathbf{P})} \sum_{l, m_{l}} \\
& \times Y_{l m_{l}}\left({\widehat{R^{\prime} \mathbf{k}}}^{*}\right) Y_{l m_{l}}^{*}\left(\widehat{R \mathbf{k}}^{*}\right) \mathcal{C}\left(\mathbf{P} \Lambda \mu ; R^{\prime}(\mathbf{P}-\mathbf{k}) ; R^{\prime} \mathbf{k}\right) \mathcal{C}^{*}(\mathbf{P} \Lambda \mu ; R(\mathbf{P}-\mathbf{k}) ; R \mathbf{k}) .
\end{aligned}
$$

For the case where the system is not boosted or when the system is restricted to be in an S-wave, this reduces to the number of elements in $\{\mathbf{k}\}_{\mathbf{P}}$. Otherwise, this depends on the number of elements being summed as well as the magnitude of the boost, the masses of the particles and the energy of the system. In all cases, the quantitiy can be easily evaluated 
numerically once the Clebsch-Gordan coefficients have been determined. For the systems of interest, these have been previously calculated in Ref. [186].

\subsubsection{Single channel S-wave result}

Here we consider the case where the orbital angular momentum is restricted to the S-wave. For this scenario to formally be applicable, the irrep of interest has to have strong overlap with the S-wave and all higher contributions must be vanishingly small. This is particularly relevant for the $\pi \pi$ system near threshold. At rest the LO contamination to the S-wave is due to $l=4$ and in the moving frame the NLO contamination is due to $l=2$, both of which are suppressed at low energies. In this scenario $\mathbb{M}$ is a one by one matrix and its adjugate is one, using Eq. 4.56 one obtains that the residue at the $n t h$ pole is

$$
R_{S, n}=\left.\left[\partial \mathbb{M} / \partial P_{0, M}\right]^{-1}\right|_{P_{0, M}=E_{\Lambda, n}}=\left[\left.\frac{8 \pi E_{n}^{*}}{\xi q_{n}^{*}} \frac{1}{\cos ^{2} \delta_{S}}\left[\frac{\partial\left(\delta_{S}+\phi_{00}\right)}{\partial P_{0, M}}\right]\right|_{P_{0, M}=E_{\Lambda, n}}\right]^{-1}
$$

where we have introduced the pseudophase $\phi_{l m}^{\mathbf{d}}$ with $(l m)$ angular momentum in the moving frame

$$
q_{\Lambda, n}^{*} \cot \phi_{l m}^{\mathbf{d}}=-\frac{4 \pi}{q_{\Lambda, n}^{* l}} c_{l m}^{\mathbf{d}}\left(q_{\Lambda, n}^{* 2} ; L\right)
$$

As seen in Eq. 4.58, the overlap of two body interpolating operator also depends on the off-shell K-matrix, where either the incoming state or outgoing is on-shell while the other state remains off-shell. In general, in this limit the $l$ th spherical harmonic decomposition of the K-matrix can be written as a function of the total energy and momentum of the system and the magnitude of the off-shell momentum in the c.m. frame, $\mathbb{K}_{l, o f f, o n}\left[(E, \mathbf{P}), p_{o f f}^{*}\right]$. For instance, when the system has zero boost, then $p_{o f f}^{*}$ would corresponding to a free momentum $2 \pi|\mathbf{n}| / L$, where $\mathbf{n}$ is an integer triplet. Similarly, one can define the spherical harmonic decomposition of the $\mathbb{Y}$ Eq.4.46. By suppressing the arguments of these quantities and considering the limit where only the S-wave contributes, we find the following overlap for the two-body operator with the $n t h$ eigenstates of the finite volume Hamiltonian

$$
\left|\left\langle 0\left|\mathcal{O}_{\Lambda \mu}(0, \mathbf{P})\right| E_{\Lambda, n} \mathbf{P} ; L\right\rangle\right|=L^{3 / 2}\left(\frac{\xi q_{n}^{*}}{8 \pi E_{n}^{*}} \frac{\mathcal{N}_{\Lambda}^{\star} \mathbb{Y}_{S} \mathbb{Y}_{S}^{\dagger} \cos ^{2} \delta_{S}}{\left.\left[\partial\left(\delta_{S}+\phi_{00}\right) / \partial P_{0, M}\right]\right|_{P_{0, M}=E_{\Lambda, n}}}\right)^{1 / 2}
$$


We note that the off-shell functions, strongly depend on the operator used in the determination of the correlation function, but as will be shown in Section 4.2 , the exact form of these functions do not matter. What does matter is that one uses the same two-body operators for the two-point correlation functions as in the three-point correlation functions. It is only in this case that the dependence on the off-shell scattering amplitudes cancels. When restricted to the $\mathrm{S}$-wave channel, $\mathcal{N}_{\Lambda}^{\star}$ is just equal to the number of momenta being summed over. Although it might be naively surprising that the matrix element of the two-particle operator depends on the off-shell scattering amplitude, this is the mechanism that is responsible for making an operator with off-shell momenta have overlap with a state which, by the definition, is on-shell. Lastly, it is worth mentioning that this result clearly explains why if one constructs an operators with a particular set of discrete momenta the resulting correlation function will have largest overlap with the nearest eigenstate. This is because the amplitude of each exponential scales as $\sim \sqrt{\left|\mathbb{Y}_{S}\right|^{2}}$. From the definition of this, Eq. 4.46, one observes that they divergent in the limit that the free energy given to the two-particle operator, $E_{f r e e}$, coincides with the on-shell energy, $E_{\Lambda, n}$. In fact, one can

shown that near this pole, the overlap factor scales as $\sim\left|E_{\Lambda, n}-E_{\text {free }}\right|^{-1}$. In Section 4.2 .4 we show that this result reproduces the well known LL-factor in a moving frame.

\subsection{2 $\pi \pi$ in a P-wave}

In the case that the two particles of interest are degenerate, parity is still a good quantum number, even in when the total momentum is nonzero. As a result, odd and even partial waves in the $\pi \pi$ systems do not mix. Therefore, when interested in studying scattering in the $\mathrm{P}$-wave $\pi \pi$ channel, the LO partial wave contamination to consider is due to the F-wave. By neglecting this contribution, $\mathbb{M}$ can be written as a one by one matrix, and the quantization condition can be in general be written as

$$
\cot \delta_{P}+\left(\cot \phi_{00}^{\mathbf{d}}+\alpha_{20, \Lambda}^{\mathbf{d}} \cot \phi_{20}^{\mathbf{d}}+\alpha_{22, \Lambda}^{\mathbf{d}} \cot \phi_{22}^{\mathbf{d}}\right)=0
$$

For systems with $\mathbf{d}=\mathbf{0}$ and cubic volumes, the $c_{2 m}^{\mathbf{d}}$ exactly vanish. For systems with non-zero total momenta or for asymmetric volumes, $c_{2 m}^{\mathbf{d}}$ do not necessarily vanish and the values of $\alpha_{20, \Lambda}^{\mathbf{d}}$ and $\alpha_{22, \Lambda}^{\mathbf{d}}$ for $\mathbf{d}^{2} \leq 3$ are shown in Table 4.2 


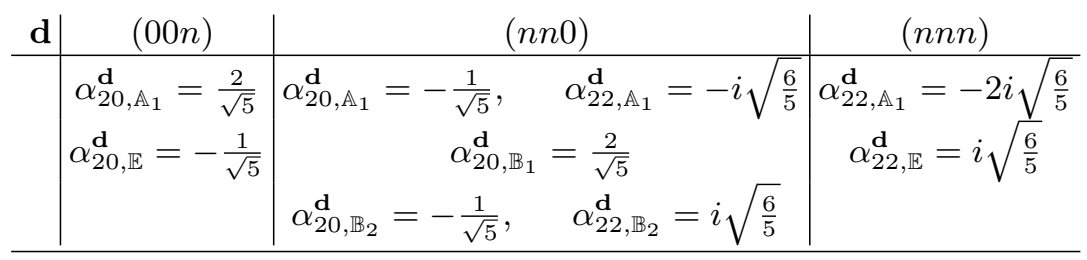

Table 4.2: Nonzero values of $\alpha_{20, \Lambda}^{\mathbf{d}}$ and $\alpha_{22, \Lambda}^{\mathbf{d}}$ for $\mathbf{d}^{2} \leq 3$. For the $\mathbb{T}_{1}^{-}$irrep of $O_{h}^{D}$, the $c_{2 m}^{\mathbf{d}}$ vanish, therefore there is no need to define $\alpha_{2 m, \Lambda}^{\mathbf{d}}$ for this irrep.

Following the steps that led to Eq. 4.62, one finds that the overlap of the two-particle interpolating operator with the $n t h$ finite volume eigenstate for a two-particle systems in a $\mathrm{P}$-wave is equal to

$$
\begin{aligned}
\left|\left\langle 0\left|\mathcal{O}_{\Lambda \mu}(0, \mathbf{P})\right| E_{\Lambda, n} \mathbf{P} ; L\right\rangle\right| & =L^{3 / 2}\left(\frac{\xi q_{n}^{*}}{8 \pi E_{n}^{*}} \mathcal{N}_{\Lambda}^{\star} \mathbb{Y}_{P} \mathbb{Y}_{P}^{\dagger} \cot ^{2} \delta_{P}\right)^{1 / 2} \\
\times & {\left.\left[\csc ^{2} \delta_{P} \frac{\partial \delta_{P}}{\partial P_{0, M}}+\csc ^{2} \phi_{00}^{\mathbf{d}} \frac{\partial \phi_{00}^{\mathbf{d}}}{\partial P_{0, M}}+\sum_{m=0,2} \alpha_{2 m, \Lambda} \csc ^{2} \phi_{2 m}^{\mathbf{d}} \frac{\partial \phi_{2 m}^{\mathbf{d}}}{\partial P_{0, M}}\right]\right|_{P_{0, M}=E_{\Lambda, n}} ^{-1 / 2} }
\end{aligned}
$$

Again, we find that this overlap factor depends on the derivative of the $\mathrm{P}$-wave phase shift and the pseudo phase $\phi_{00}^{\mathbf{d}}$, but also depends nontrivially on the derivative of the pseudo phases $\phi_{20}^{\mathbf{d}}$ and $\phi_{22}^{\mathbf{d}}$. Note that for a system composed of indistinguishable particles, such as the $\pi \pi$ system, one must set the symmetry factor $\xi$ equal to $1 / 2$. In Section 4.2 .4 we show this leads to the needed LL-factor for $\pi \gamma \rightarrow \pi \pi$ when the final state is in a P-wave.

\subsection{3 $\pi K$ for $J \leq 1$}

As a slightly more complicated example, we consider the $\pi K$ operator. For such system with zero total momentum, parity is a good quantum number and as a result odd and even partial waves do not mix. If we restrict the angular momentum to satisfy $J \leq 1$, the system could be in a S- or P-wave. The corresponding cubic irreps would be the $\mathbb{A}_{1}^{+}$and $\mathbb{T}_{1}^{-}$, and the matrix elements of their respective operators are described by Eqs. $4.62 \& 4.64$, respectively. 
For the $\pi K$ systems, $\xi$ must be set to one. For boosted system, parity is non longer a good quantum number. As a result odd and even partial waves will mix. By neglecting D-wave contamination, one can observe that for boosted systems at least one irreps will have large overlap with $\mathrm{P}$-wave states and no overlap with the S-wave. One can readily identify such irreps as $\mathbb{E}$ for $\mathbf{d}=(00 n), \mathbb{B}_{1}$ and $\mathbb{B}_{2}$ for $\mathbf{d}=(n n 0)$, and $\mathbb{E}$ for $\mathbf{d}=(n n n)$. For these irreps, the overlap factor is again shown in Eq. 4.64. The non-vanishing values for $\alpha_{20, \Lambda}^{\mathbf{d}}$ and $\alpha_{22, \Lambda}^{\mathbf{d}}$ for $\mathbf{d}^{2} \leq 3$ are given in Table 4.2 . The $\mathbb{A}_{1}$ irrep for these boost vectors will be an admixture of $\mathrm{S}$ - and $\mathrm{P}$-wave. As an example, consider the $\mathbb{A}_{1}$ in the $\mathrm{Dic}_{4}$ group, namely the symmetry group for $\mathbf{d}=(00 n)$. This irrep mixes the $(l, m)=\{(0,0),(1,0), \ldots\}$ partial waves. In this space one can write down the finite volume function $\mathbb{F}^{V}$ and the on-shell/off-shell K-matrices for this irrep as

$$
\begin{aligned}
& \operatorname{Dic}_{4} \mathbb{A}_{1}: \quad \quad \mathbb{F}_{\mathbb{A}_{1}}^{V}=\frac{q_{\mathbb{A}_{1}, o n}^{*}}{8 \pi E_{\mathbb{A}_{1}}^{*}}\left(\begin{array}{cc}
\cot \phi_{00}^{\mathbf{d}} & \cot \phi_{10}^{\mathbf{d}} \\
\cot \phi_{10}^{\mathbf{d}} & \cot \phi_{00}^{\mathbf{d}}+2 / \sqrt{5} \cot \phi_{20}^{\mathbf{d}}
\end{array}\right), \\
& \mathbb{K}_{\text {on,on; } \mathbb{A}_{1}}=\frac{8 \pi E_{\mathbb{A}_{1}}^{*}}{q_{\mathbb{A}_{1}, \text { on }}^{*}}\left(\begin{array}{cc}
{\left[\cot \delta_{S}\right]^{-1}} & 0 \\
0 & {\left[\cot \delta_{P}\right]^{-1}}
\end{array}\right) \text {, } \\
& \mathbb{K}_{o n, o f f ; \mathbb{A}_{1}}=\left(\begin{array}{cc}
\mathbb{K}_{o n, o f f ; S} & 0 \\
0 & \mathbb{K}_{o n, o f f ; P}
\end{array}\right)
\end{aligned}
$$

The quantization condition can be written as

$$
\operatorname{Dic}_{4} \mathbb{A}_{1}: \operatorname{det}\left[\mathbb{M}_{\mathbb{A}_{1}}\right]=\operatorname{det}\left[\mathbb{K}_{o n, o n ; \mathbb{A}_{1}}+\left(\mathbb{F}_{\mathbb{A}_{1}}^{V}\right)^{-1}\right]=0 .
$$

In order to evaluate $\left|\left\langle 0\left|\mathcal{O}_{\mathbb{A}_{1}, 0, \mathbf{P}}\right| E_{\mathbb{A}_{1}, n} \mathbf{P} ; L\right\rangle\right|$, we first need to evaluate the adjugate of $\mathbb{M}_{\mathbb{A}_{1}}$,

$$
\operatorname{adj}\left[\mathbb{M}_{\mathbb{A}_{1}}\right]=\left(\begin{array}{cc}
{\left[\mathbb{M}_{\mathbb{A}_{1}}\right]_{22}} & -\left[\mathbb{M}_{\mathbb{A}_{1}}\right]_{12} \\
-\left[\mathbb{M}_{\mathbb{A}_{1}}\right]_{21} & {\left[\mathbb{M}_{\mathbb{A}_{1}}\right]_{11}}
\end{array}\right),
$$

and we define the Clebsch-Gordan coefficients as vectors in the spherical harmonic basis,

$$
\mathbb{C}_{\mathbb{A}_{1}}=\sqrt{4 \pi} \sum_{R \in \mathrm{LG}(\mathbf{P})} \mathcal{C}\left(\mathbf{P} \mathbb{A}_{1} ; R(\mathbf{P}-\mathbf{k}) ; R \mathbf{k}\right)\left(\begin{array}{c}
Y_{00}\left(\widehat{R \mathbf{k}}^{*}\right) \\
Y_{10}\left(\widehat{R \mathbf{k}}^{*}\right)
\end{array}\right) .
$$


we obtain the overlap factor for the $\mathbb{A}_{1}$ irrep for the Dic 4 group as follows,

$$
\left|\left\langle 0\left|\mathcal{O}_{\mathbb{A}_{1} \mu, 0, \mathbf{P}}\right| E_{\mathbb{A}_{1}, n} \mathbf{P} ; L\right\rangle\right|=L^{3 / 2} \frac{\left(\mathbb{C}_{\mathbb{A}_{1}}^{T} \mathbb{K}_{o f f, o n ; \mathbb{A}_{1}} \mathbb{Y}_{\mathbb{A}_{1}} \operatorname{adj}\left[\mathbb{M}_{\mathbb{A}_{1}}\right] \mathbb{Y}_{\mathbb{A}_{1}}^{\dagger} \mathbb{C}_{\mathbb{A}_{1}}^{*}\right)^{1 / 2}}{\operatorname{tr}\left[\operatorname{adj}\left[\mathbb{M}_{\mathbb{A}_{1}}\right] \frac{\partial \mathbb{M}_{\mathbb{A}_{1}}}{\partial E_{\mathbb{A}_{1}, n}}\right]^{1 / 2}}
$$

Similar expressions can be found for the $\mathbb{A}_{1}$ irreps of the $\mathrm{Dic}_{2}$ and $\mathrm{Dic}_{3}$ groups, the only differences would be the values of the finite volume functions and the K-matrices appearing in Eqs. 4.68 \& 4.67 and the Clebsch-Gordan coefficients appearing in Eq. 4.70. For example, the $\mathbb{A}_{1}$ irrep of the $\operatorname{Dic}_{2}$ mixes the $(l, m)=\{(0,0),(1,-1),(1,1), \ldots\}$ partial waves.

$\operatorname{Dic}_{2} \mathbb{A}_{1}:$

$$
\begin{aligned}
& \mathbb{F}_{\mathbb{A}_{1}}^{V}=\frac{q_{\mathbb{A}_{1}, \text { on }}^{*}}{8 \pi E_{\mathbb{A}_{1}}^{*}}\left(\begin{array}{ccc}
\cot \phi_{00}^{\mathbf{d}} & i^{3 / 2} \operatorname{Re}\left[\cot \phi_{11}^{\mathbf{d}}\right] & i^{1 / 2} \operatorname{Re}\left[\cot \phi_{11}^{\mathbf{d}}\right] \\
-i^{1 / 2} \operatorname{Re}\left[\cot \phi_{11}^{\mathbf{d}}\right] & \cot \phi_{00}^{\mathbf{d}}-\cot \phi_{20}^{\mathbf{d}} / \sqrt{5} & -\sqrt{6 / 5} \cot \phi_{22}^{\mathbf{d}} \\
-i^{3 / 2} \operatorname{Re}\left[\cot \phi_{11}^{\mathbf{d}}\right] & \sqrt{6 / 5} \cot \phi_{22}^{\mathbf{d}} & \cot \phi_{00}^{\mathbf{d}}-\cot \phi_{20}^{\mathbf{d}} / \sqrt{5}
\end{array}\right), \\
& \mathbb{K}_{o n, o n ; \mathbb{A}_{1}}=\frac{8 \pi E_{\mathbb{A}_{1}}^{*}}{q_{\mathbb{A}_{1}, \text { on }}^{*}}\left(\begin{array}{ccc}
{\left[\cot \delta_{S}\right]^{-1}} & 0 & 0 \\
0 & {\left[\cot \delta_{P}\right]^{-1}} & 0 \\
0 & 0 & {\left[\cot \delta_{P}\right]^{-1}}
\end{array}\right) \text {, } \\
& \mathbb{K}_{\text {on,off; } ; \mathbb{A}_{1}}=\left(\begin{array}{ccc} 
& & \\
\mathbb{K}_{\text {on,off;S }} & 0 & 0 \\
0 & \mathbb{K}_{\text {on }, o f f ; P} & 0 \\
0 & 0 & \mathbb{K}_{\text {on,off;P }}
\end{array}\right) \text {. }
\end{aligned}
$$

Similarly, one can write down the Clebsch-Gordan coefficients as a three-dimensional vector in this space,

$$
\mathbb{C}_{\mathbb{A}_{1}}=\sqrt{4 \pi} \sum_{R \in \mathrm{LG}(\mathbf{P})} \mathcal{C}\left(\mathbf{P} \mathbb{A}_{1} ; R(\mathbf{P}-\mathbf{k}) ; R \mathbf{k}\right)\left(\begin{array}{c}
Y_{00}\left(\widehat{R \mathbf{k}}^{*}\right) \\
Y_{1-1}\left(\widehat{R \mathbf{k}}^{*}\right) \\
Y_{11}\left(\widehat{R \mathbf{k}}^{*}\right)
\end{array}\right)
$$


The final piece needed is the evaluation of the adjugate of a three-dimensional matrix

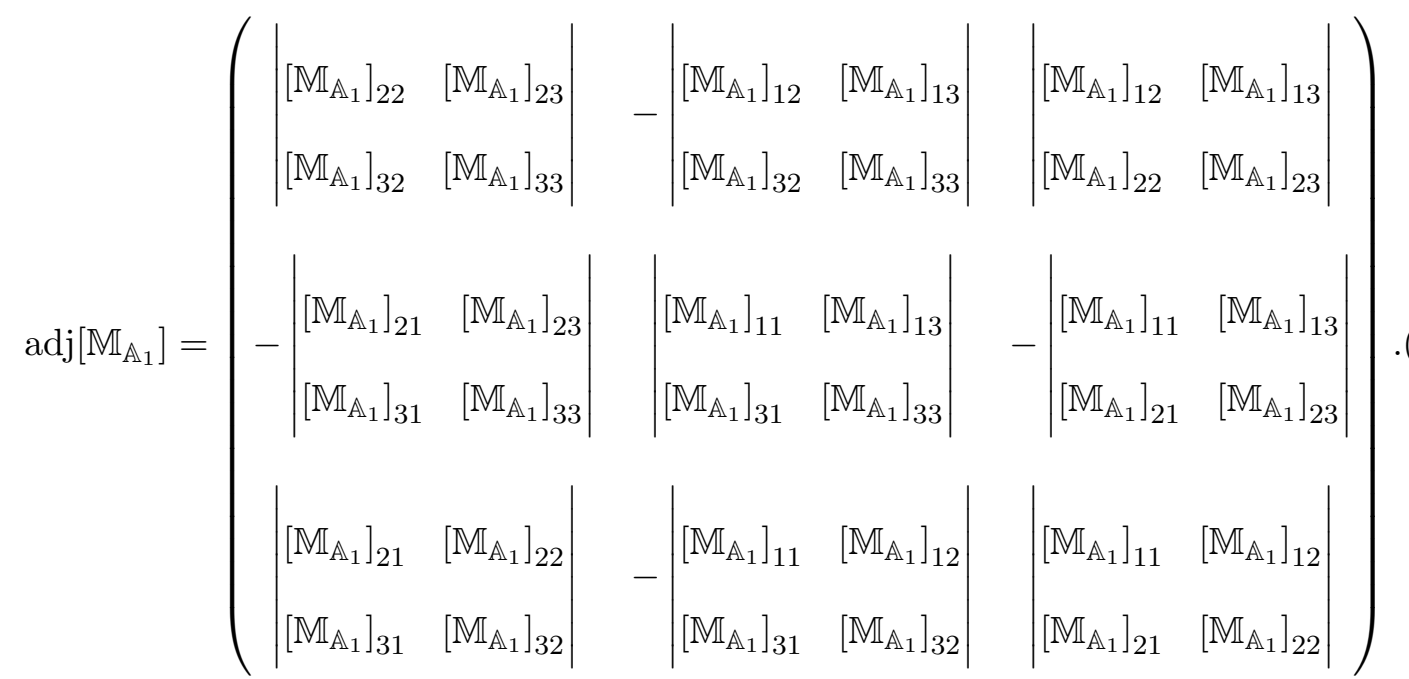

These two examples explicitly illustrate how partial wave mixing can be appropriately dealt with in numerical studies of the two-point correlation function. Similarly, one can consider the scenario where the scattering amplitudes couples different on-shell channels, in Section 4.2 .4 we discuss how to determine the LL-factor for such systems.

\subsection{Three-point correlation functions and the generalized Lellouch-Lüscher formula}

Having discussed two-point correlation functions extensively in the previous section, we now proceed to the main focus of this work, three-point correlation functions. In particular, we are interested in processes where an external current annihilates a single-particle state and creates a two-particle state. Such a transition was first considered in this context by Lellouch and Lüscher, who derived a relation between a finite-volume matrix element and the physical decay rate for $K \rightarrow \pi \pi$ [70]. The weak Hamiltonian is the external current in that process, and thus the analysis is restricted to scalar currents which insert zero momentum. Here we extend the result by allowing the external current to inject arbitrary four momentum and to be in any irrep of the finite-volume symmetry group. This is particularly relevant for meson photoproduction processes such as $\pi \gamma \rightarrow \pi \pi$ as well as meson decays of the form $\phi_{1} \rightarrow \phi_{2} \phi_{3} X$, where $X$ denotes an arbitrary leptonic current. Even the relatively simple example of $\pi \gamma \rightarrow \pi \pi$ illustrates that the finite-volume final state 
mixes different angular momenta, due to the reduction of rotational symmetry, as well as states with different particle content. For example the $\pi \pi$ state mixes with $K \bar{K}$ as well as $\pi \pi \pi \pi$, etc 13 Following the discussion of the previous section, we accommodate any number of strongly-coupled channels, but restrict attention to energies for which only two-particle states can go on-shell.

\subsubsection{Construction of currents in irreps of $L G(\boldsymbol{Q})$}

In order to construct the three-point correlation function, we must first define currents in irreps of the finite-volume symmetry groups. We begin by defining a current of interest in the infinite-volume theory. As a specific example, consider a four-vector current which couples an incoming single-particle state, with momentum $P_{i}$, to an outgoing (asymptotic) two-particle state, where one particle has momentum $k$ and the other $P_{f}-k$. Defining $h_{\nu}\left(P_{i}, P_{f}-k, k\right)$ as the LO transition amplitude for this process, we introduce

$$
\begin{aligned}
\mathcal{J}_{\nu}(x)=\frac{\xi}{L^{9}} \sum_{\mathbf{P}_{f}, \mathbf{k}, \mathbf{P}_{i}} \int \frac{d P_{f, 0}}{2 \pi} \frac{d P_{i, 0}}{2 \pi} & \frac{d k_{0}}{2 \pi} e^{i\left(P_{i}-P_{f}\right) \cdot x} \\
& \times \bar{\varphi}^{\dagger}\left(-P_{f}+k\right) \tilde{\varphi}^{\dagger}(-k) \varphi\left(P_{i}\right) h_{\nu}\left(P_{i}, P_{f}-k, k\right) .
\end{aligned}
$$

Here $\xi=1 / 2$ if $\bar{\varphi}=\widetilde{\varphi}$ and otherwise $\xi=1$. The zero component of this four-vector current transforms trivially under rotations, also within the finite-volume subgroups. By contrast, the spatial vector (or pseudovector) is in the $J=1$ irrep of $S O(3)$, and thus transforms under multiple irreps of the finite-volume groups.

In order to discuss the subduction of the vector current onto irreps of the octahedral group and the little groups, it is convenient to first Fourier transform

$$
\begin{aligned}
& \widetilde{\mathcal{J}}_{j}\left(x_{0}, \mathbf{Q}\right)=\int d^{3} \mathbf{x} e^{-i \mathbf{Q} \cdot \mathbf{x}} \mathcal{J}_{j}(x) \\
&=\frac{\xi}{L^{6}} \sum_{\mathbf{P}_{f}, \mathbf{k}, \mathbf{P}_{i}} \int \frac{d P_{f, 0}}{2 \pi} \frac{d P_{i, 0}}{2 \pi} \frac{d k_{0}}{2 \pi} e^{i\left(P_{i, 0}-P_{f, 0}\right) x_{0}} \bar{\varphi}^{\dagger}\left(P_{f}-k\right) \widetilde{\varphi}^{\dagger}(-k) \varphi\left(P_{i}\right) \\
& \quad \times h_{j}\left(P_{i}, P_{f}-k, k\right) \delta_{\mathbf{P}_{f}, \mathbf{Q}+\mathbf{P}_{i}}
\end{aligned}
$$

\footnotetext{
${ }^{13}$ Similar to the case of $D \rightarrow\{\pi \pi, K \bar{K}\}$ decays studied in Ref. [140.
} 
and also to switch from Cartesian to spherical-harmonic basis

$$
\widetilde{\mathcal{J}}_{ \pm 1}=\mp \frac{1}{\sqrt{2}}\left(\widetilde{\mathcal{J}}_{x} \pm i \widetilde{\mathcal{J}}_{y}\right), \quad \widetilde{\mathcal{J}}_{0}=\widetilde{\mathcal{J}}_{z}
$$

For non-zero $\mathbf{Q}$, the azimuthal component of the vector current is only a good quantum number if the $\hat{z}$ axis and the momentum axis coincide. It is thus convenient to instead use operators in the helicity basis. These are found by defining $R$ as an active rotation from $(0,0,|\mathbf{Q}|)$ to $\mathbf{Q}$ and $\mathcal{D}_{m_{1} m_{2}}^{(J)}(R)$ as the $m_{1} m_{2}$ component of the corresponding Wigner- $\mathcal{D}$ matrix in the $J$ representation. With this, one can rotate from the spherical-harmonic to the helicity basis

$$
\widetilde{\mathcal{J}}_{\lambda}\left(y_{0}, \mathbf{Q}\right)=\sum_{m} \mathcal{D}_{m \lambda}^{(1) *}(R) \widetilde{\mathcal{J}}_{m}\left(y_{0}, \mathbf{Q}\right)
$$

We are now in position to decompose the current into irreps of the finite-volume symmetry groups. First restricting attention to $\widetilde{\mathcal{J}}_{\lambda}\left(y_{0}, \mathbf{0}\right)$, we comment that the current can be subduced onto the $\Lambda$ irrep of $O_{h}$ using the subduction coefficients, $\left[C_{\Lambda}^{J}\right]_{\mu, \lambda}$ [226]. As can be seen in Table 4.1(a), for this case the subduction is trivial. The $J=1$ irrep becomes the $\mathbb{T}_{1}$ irrep of the octahedral group, with each element of the helicity basis equal to one of the three $\mu$ values labeling the finite-volume counterpart. For systems in flight one may define a similar subduction. In this case nontrivial linear combinations arise, given by

$$
\tilde{\mathcal{J}}_{\Lambda \mu}^{[J, P,|\lambda|]}\left(y_{0}, \mathbf{Q}\right)=\sum_{\hat{\lambda}= \pm|\lambda|} \mathcal{S}_{\Lambda \mu}^{\widetilde{\eta} \hat{\lambda}} \tilde{\mathcal{J}}_{\hat{\lambda}}^{[J, P]}\left(y_{0}, \mathbf{Q}\right)
$$

where now $J$ and $P$ specify the angular momentum and parity of the system at rest. Table 4.1(b) shows the values of $\mathcal{S}_{\Lambda \mu}^{\widetilde{\eta} \lambda}$ for systems with integer $J \leq 2$ and $L \mathbf{Q} / 2 \pi=$ $\{(0,0, n),(n, n, 0),(n, n, n)\}$ and all other possible cubic rotations are determined in Ref. [226].

Having discussed how to subduce the vector current onto a definite irrep of $L G(\mathbf{Q})$, one can easily generalize this for currents of any rank,

$$
\widetilde{\mathcal{J}}_{\alpha, \beta, \ldots, \omega}\left(x_{0}, \mathbf{Q}\right) \longrightarrow \widetilde{\mathcal{J}}_{\Lambda \mu}^{[J, P,|\lambda|]}\left(x_{0}, \mathbf{Q}\right)
$$

The discussion that follows is relevant for arbitrary rank currents with either positive or negative parity that have been properly subduced onto the irreps of the corresponding 
symmetry group. The key point is that, by taking appropriate linear combinations, one can transform an operator in any basis to one that transforms as an irrep of the finite-volume group. The linear combinations of currents imply linear combinations of the transition

amplitudes so that both $\widetilde{\mathcal{J}}$ and $h$ may be reexpressed in terms of finite-volume irreps, and the form of Eq. 4.75 is preserved in the new basis

$$
\begin{aligned}
\widetilde{\mathcal{J}}_{\Lambda \mu}^{[J, P,|\lambda|]}\left(x_{0}, \mathbf{Q}\right)=\frac{\xi}{L^{6}} \sum_{\mathbf{P}_{f}, \mathbf{k}, \mathbf{P}_{i}} \int \frac{d P_{f, 0}}{2 \pi} \frac{d P_{i, 0}}{2 \pi} & \frac{d k_{0}}{2 \pi} e^{i\left(P_{i, 0}-P_{f, 0}\right) x_{0}} \bar{\varphi}^{\dagger}\left(-P_{f}+k\right) \widetilde{\varphi}^{\dagger}(-k) \varphi\left(P_{i}\right) \\
& \times h_{\Lambda \mu}^{[J, P,|\lambda|]}\left(P_{i}, P_{f}-k, k\right) \delta_{\mathbf{P}_{f}, \mathbf{Q}+\mathbf{P}_{i}}
\end{aligned}
$$

Finally, in order to consider scenarios where there is $N>1$ open two-particle channels, one need only generalize this expression to

$$
\begin{array}{r}
\widetilde{\mathcal{J}}_{\Lambda \mu}^{[J, P,|\lambda|]}\left(x_{0}, \mathbf{Q}\right)=\sum_{a=1}^{N} \frac{\xi_{a}}{L^{6}} \sum_{\mathbf{P}_{f}, \mathbf{k}, \mathbf{P}_{i}} \int \frac{d P_{f, 0}}{2 \pi} \frac{d P_{i, 0}}{2 \pi} \frac{d k_{0}}{2 \pi} e^{i\left(P_{i, 0}-P_{f, 0}\right) x_{0}} \bar{\varphi}_{a}^{\dagger}\left(-P_{f}+k\right) \widetilde{\varphi}_{a}^{\dagger}(-k) \varphi\left(P_{i}\right) \\
\times h_{\Lambda \mu}^{[J, P,|\lambda|]}\left(P_{i}, P_{f}-k, k, a\right) \delta_{\mathbf{P}_{f}, \mathbf{Q}+\mathbf{P}_{i}},
\end{array}
$$

where $\bar{\varphi}_{a}^{\dagger}$ and $\widetilde{\varphi}_{a}^{\dagger}$ create the two particles in the ath channel and $h_{\Lambda \mu}^{[J, P,|\lambda|]}\left(P_{i}, P_{f}-k, k, a\right)$ is the LO transition amplitude for that channel.

\subsubsection{Three-point correlation function}

Having properly defined the current of interest, we proceed to evaluate three-point correlation functions. Arriving at the result with an arbitrary number of open two-particle states is straightforward after one determines the single-channel result. We thus suppress the channel index for the time being and use Eq. 4.81 for the current. We begin by writing down the analogous expression to Eq. 4.19, when a current with arbitrary momentum is inserted at time $t=y_{0}$,

$$
\begin{aligned}
C_{\Lambda_{f} \mu_{f} ; \Lambda \mu}^{(1 \rightarrow 2)}\left(x_{f, 0}-\right. & \left.y_{0} ; y_{0}-x_{i, 0}\right)=\left\langle 0\left|\mathcal{O}_{\Lambda_{f} \mu_{f}}\left(x_{f, 0}, \mathbf{P}_{f}\right) \widetilde{\mathcal{J}}_{\Lambda \mu}^{[J, P,|\lambda|]}\left(y_{0}, \mathbf{Q}\right) \varphi^{\dagger}\left(x_{i, 0},-\mathbf{P}_{i}\right)\right| 0\right\rangle \\
= & \sum_{n_{f}} e^{-E_{\Lambda_{f}, n_{f}}\left(x_{f, 0}-y_{0}\right)} e^{-E_{\Lambda_{i}, 0}\left(y_{0}-x_{i, 0}\right)}\left\langle 0\left|\mathcal{O}_{\Lambda_{f} \mu_{f}}\left(0, \mathbf{P}_{f}\right)\right| E_{\Lambda_{f}, n_{f}} \mathbf{P}_{f} ; L\right\rangle \\
& \times\left\langle E_{\Lambda_{f}, n_{f}} \mathbf{P}_{f} ; L\left|\widetilde{\mathcal{J}}_{\Lambda \mu}^{[J, P,|\lambda|]}(0, \mathbf{Q})\right| E_{\Lambda_{i}, 0} \mathbf{P}_{i} ; L\right\rangle\left\langle E_{\Lambda_{i}, 0} \mathbf{P}_{i} ; L\left|\varphi^{\dagger}\left(0,-\mathbf{P}_{i}\right)\right| 0\right\rangle .
\end{aligned}
$$

In the second line we have assumed $x_{i, 0}<y_{0}<x_{f, 0}$. 


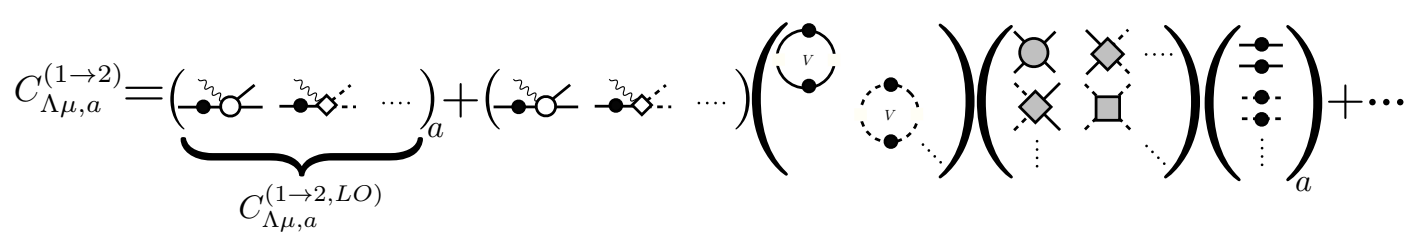

(a)

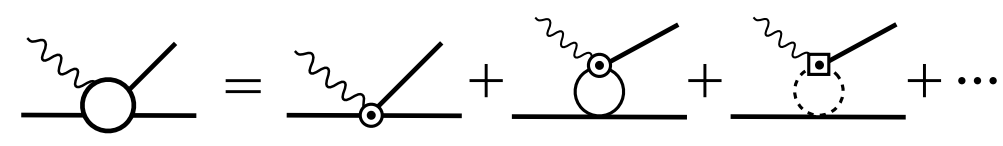

(b)

Figure 4.3: a) Diagramatic representation for the three-point correlation function for processes involving a single incoming particle and outgoing two-particle state. This is written in terms of the LO transition amplitudes, one of which is explicit shown in (b), and the Bethe-Salpeter kernel, depicted in Fig. 4.1(b). The wiggly line is meant to depict an integer spin external current that can inject arbitrary four-momenta. Note that disconnected diagrams appearing in the LO transition amplitudes vanish except in the case where the current has the same quantum numbers as one of the outgoing external legs. 
In order to get insight as to how one can interpret $\left\langle E_{\Lambda_{f}, n_{f}} \mathbf{P}_{f} ; L\left|\widetilde{\mathcal{J}}_{\Lambda \mu}^{[J, P,|\lambda|]}(0, \mathbf{Q})\right| E_{\Lambda_{i}, 0} \mathbf{P}_{i} ; L\right\rangle$, we also evaluate the correlation function diagrammatically, as depicted in Fig. $4 .{ }^{14}$ First observe that the transition amplitude, shown in Fig. 4.3(b), is defined in analogy to the Bethe-Salpeter kernel as the sum of all amputated diagrams that are two particle irreducible in the s-channel. The object differs from the Bethe-Salpeter only in the form of external legs and in the insertion of a new contact interaction associated with the electroweak process of interest. To evaluate the three-point correlator we must sum all diagrams that appear when the external legs of the transition amplitude are contracted with the single incoming particle and outgoing two-particle state. We perform the calculation of the three-point correlator in two steps, first considering the contraction of the incoming state with the current

$$
\begin{aligned}
\mathcal{D}^{(1)}\left(y_{0}-x_{i, 0}\right)= & \frac{1}{L^{3}} \sum_{\mathbf{P}_{i^{\prime}}} \int \frac{d P_{i^{\prime}, 0}}{2 \pi} e^{i P_{i^{\prime}, 0} y_{0}}\left\langle\varphi\left(P_{i^{\prime}}\right) \varphi^{\dagger}\left(x_{i, 0},-\mathbf{P}_{i}\right)\right\rangle \\
& \times h_{\Lambda \mu}^{[J, P,|\lambda|]}\left(P_{i^{\prime}}, P_{f}-k_{f^{\prime}}, k_{f^{\prime}}\right) \delta_{\mathbf{P}_{f}, \mathbf{Q}+\mathbf{P}_{i^{\prime}}} \\
= & \left(\frac{e^{-\left(y_{0}-x_{i, 0}\right) E_{\Lambda_{i}, 0}}}{2 E_{\Lambda_{i}, 0}}\right) h_{\Lambda \mu}^{[J, P,|\lambda|]}\left(P_{i}, P_{f}-k_{f^{\prime}}, k_{f^{\prime}}\right) \delta_{\mathbf{P}_{f}, \mathbf{Q}+\mathbf{P}_{i}} \\
& +\mathcal{O}\left(e^{-E_{3, t h}\left(y_{0}-x_{i, 0}\right)} / E_{3, t h}\right)
\end{aligned}
$$

where $P_{i, 0}=i E_{\Lambda_{i}, 0}$. The remaining contractions, between the current and the final twoparticle operator, give

$$
\begin{aligned}
\mathcal{D}^{(2)}\left(x_{f, 0}-y_{0}\right)= & \frac{\xi}{L^{3}} \sum_{\mathbf{P}_{f}, \mathbf{k}_{f}} \int \frac{d P_{f, 0}}{2 \pi} \frac{d k_{f, 0}}{2 \pi} e^{-i P_{f} y_{0}}\left\langle\mathcal{O}_{\Lambda_{f} \mu_{f}}\left(x_{f}, \mathbf{P}_{f}\right) \bar{\varphi}^{\dagger}\left(-P_{f}+k_{f}\right) \widetilde{\varphi}^{\dagger}\left(-k_{f}\right)\right\rangle \\
& \times h_{\Lambda \mu}^{[J, P,|\lambda|]}\left(P_{i}, P_{f}-k_{f}, k_{f}\right) \delta_{\mathbf{P}_{f}, \mathbf{Q}+\mathbf{P}_{i}} .
\end{aligned}
$$

The LO contribution of this term is found to be

$$
\begin{aligned}
\mathcal{D}^{(2, L O)}\left(x_{f, 0}-y_{0}\right)=L^{3} & \int \frac{d P_{f, 0}}{2 \pi} e^{i P_{f, 0}\left(x_{f, 0}-y_{0}\right)} \sum_{R \in \mathrm{LG}\left(\mathbf{P}_{\mathrm{f}}\right)} \mathcal{C}\left(\mathbf{P}_{f} \Lambda_{f} \mu_{f} ; R\left(\mathbf{P}_{f}-\mathbf{k}_{f}\right) ; R \mathbf{k}_{f}\right) \\
& \times \frac{h_{\Lambda \mu}^{[J, P,|\lambda|]}\left(P_{i}, P_{f}-k_{f}, k_{f}\right)}{4 \omega_{1} \omega_{2}\left(i P_{f, 0}+\left(\omega_{1}+\omega_{2}\right)\right)} \delta_{\mathbf{P}_{f}, \mathbf{Q}+\mathbf{P}_{i}+\cdots}
\end{aligned}
$$

\footnotetext{
${ }^{14}$ Note that Fig. 4.3 shows the expression for the correlation function when arbitrary number of final two-particle states are present. The single channel scenario is recovered by suppressing the dependence on the $a$ index and reducing all matrices in the channel space to scalars.
} 
where the ellipses denote contributions associated with higher energy poles of the free twoparticle propagator. Note that the symmetry factor cancels.

To complete our calculation of $C^{(1 \rightarrow 2)}$, it remains only to include all higher order corrections to $\mathcal{D}^{(2)}$. These arrise from insertions of the Bethe-Salpeter kernel between the current and the two-body operator. All contributions are included by making the substitution

$$
\begin{aligned}
& h_{\Lambda \mu}^{[J, P,|\lambda|]}\left(P_{i}, P_{f}-k_{f}, k_{f}\right) \longrightarrow h_{\Lambda \mu}^{[J, P,|\lambda|]}\left(P_{i}, P_{f}-k_{f}, k_{f}\right) \\
& -\frac{\xi}{L^{3}} \sum_{\mathbf{k}_{f^{\prime}}} \frac{\mathbb{T}_{L}\left(P_{f}, k_{f^{\prime}}, k_{f}\right) h_{\Lambda \mu}^{[J, P,|\lambda|]}\left(P_{i}, P_{f}-k_{f^{\prime}}, k_{f^{\prime}}\right)}{4 \omega_{1, \mathbf{P}_{f}-\mathbf{k}_{f^{\prime}}} \omega_{2, \mathbf{k}_{f^{\prime}}}\left(\omega_{1, \mathbf{P}_{f}-\mathbf{k}_{f^{\prime}}}+\omega_{2, \mathbf{k}_{f^{\prime}}}+i P_{f, 0}\right)}+\cdots,
\end{aligned}
$$

where the ellipses again denote higher energy poles.

To give the final result we must first define $\mathbb{H}_{\Lambda \mu}^{[J, P,|\lambda|]}\left(P_{i}, P_{f}-k_{f}, k_{f}\right)$ as the sum over all infinite-volume diagrams contributing to the transition amplitude, evaluated using the principal-value prescription (as depicted in Fig. 4.4(b) for a single channel). This is also given by

$$
\begin{aligned}
\mathbb{H}_{\Lambda \mu}^{[J, P,|\lambda|]}\left(P_{i}, P_{f}\right. & \left.-k_{f}, k_{f}\right) \equiv h_{\Lambda \mu}^{[J, P,|\lambda|]}\left(P_{i}, P_{f}-k_{f}, k_{f}\right) \\
& +\xi \int_{\mathbf{k}_{f^{\prime}}} \text { P.V. } \frac{\mathbb{K}_{o f f, o f f}\left(P_{f}, k_{f^{\prime}}, k_{f}\right) h_{\Lambda \mu}^{[J, P,|\lambda|]}\left(P_{i}, P_{f}-k_{f^{\prime}}, k_{f^{\prime}}\right)}{4 \omega_{1, \mathbf{P}_{f}-\mathbf{k}_{f^{\prime}}} \omega_{2, \mathbf{k}_{f^{\prime}}}\left(\omega_{1, \mathbf{P}_{f}-\mathbf{k}_{f^{\prime}}}+\omega_{2, \mathbf{k}_{f^{\prime}}}+i P_{f, 0}\right)}+\cdots
\end{aligned}
$$

In addition we define $\mathbb{H}_{l m ; \Lambda \mu}=\int d \Omega Y_{l m_{l}}^{*}\left(\hat{\mathbf{k}}_{f}^{*}\right) \mathbb{H}_{\Lambda \mu}^{[J, P,|\lambda|]}\left(P_{i}, P_{f^{\prime}}-k_{f}, k_{f}\right)$, which is the projection of this amplitude onto the spherical harmonic basis of the outgoing state. Note that this requires evaluating the transition amplitude in the frame where the final two-particle state is at rest.

Putting all the pieces together and performing the integral over $P_{f, 0}$, one finds the 
following expressions for the three-point correlation function

$$
\begin{aligned}
& C_{\Lambda_{f} \mu_{f} ; \Lambda \mu}^{(1 \rightarrow 2)}\left(x_{f, 0}-y_{0} ; y_{0}-x_{i, 0}\right)=\left(\frac{e^{-\left(y_{0}-x_{i, 0}\right) E_{\Lambda_{i}, 0}}}{2 E_{\Lambda_{i}, 0}}\right) \int \frac{d P_{f, 0}}{2 \pi} e^{i P_{f, 0}\left(x_{f, 0}-y_{0}\right)} \delta_{\mathbf{P}_{f}, \mathbf{Q}+\mathbf{P}_{i}}
\end{aligned}
$$

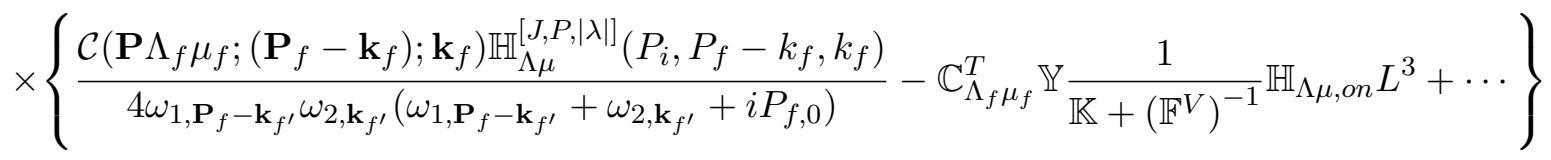

$$
\begin{aligned}
& =\left(\frac{e^{-\left(y_{0}-x_{i, 0}\right) E_{\Lambda_{i}, 0}}}{2 E_{\Lambda_{i}, 0}}\right) L^{3} \sum_{n_{f}} e^{-E_{\Lambda_{f}, n_{f}}\left(x_{f, 0}-y_{0}\right)} \mathbb{C}_{\Lambda_{f} \mu_{f}}^{T} \mathbb{Y}_{\Lambda_{f}, n_{f}} R_{\Lambda_{f}, n_{f}} \mathbb{H}_{\Lambda_{f}, n_{f} ; \Lambda \mu} \delta_{\mathbf{P}_{f}, \mathbf{Q}+\mathbf{P}_{i}} \\
& +\cdots,
\end{aligned}
$$

where the ellipses denote contribution from high energy poles. Note that, just like in the two-point correlation function, the free-particle poles do not contribute due to the careful cancelation of the two objects inside the braces.

By comparing Eqs. $4.83 \& 4.90$ and multiplying with the complex conjugate expression, one finds an identity for the finite-volume matrix element

$$
\begin{aligned}
& \left|\left\langle E_{\Lambda_{f}, n_{f}} \mathbf{P}_{f} ; L\left|\widetilde{\mathcal{J}}_{\Lambda \mu}^{[J, P,|\lambda|]}\left(0, \mathbf{P}_{f}-\mathbf{P}_{i}\right)\right| E_{\Lambda_{i}, 0} \mathbf{P}_{i} ; L\right\rangle\right| \\
& =\left(\frac{L^{3}}{2 E_{\Lambda_{i}, 0}}\right) \frac{\sqrt{\left(\mathbb{C}_{\Lambda_{f} \mu_{f}}^{T} \mathbb{Y}_{\Lambda_{f}, n_{f}} R_{\Lambda_{f}, n_{f}} \mathbb{H}_{\Lambda_{f}, n_{f} ; \Lambda \mu}\right)\left(\mathbb{H}_{\Lambda_{f}, n_{f} ; \Lambda \mu}^{T} R_{\Lambda_{f}, n_{f}} \mathbb{Y}_{\Lambda_{f}, n_{f}}^{\dagger} \mathbb{C}_{\Lambda_{f} \mu_{f}}^{*}\right)}}{\left|\left\langle 0\left|\mathcal{O}_{\Lambda_{\mu}}\left(0, \mathbf{P}_{f}\right)\right| E_{\Lambda_{f}, n_{f}} \mathbf{P}_{f} ; L\right\rangle\right|\left|\left\langle E_{\Lambda_{i}, 0} \mathbf{P}_{i} ; L\left|\varphi^{\dagger}\left(0,-\mathbf{P}_{i}\right)\right| 0\right\rangle\right|} \\
& =\frac{1}{\sqrt{2 E_{\Lambda_{i}, 0}}} \sqrt{\frac{\left(\mathbb{C}_{\Lambda_{f} \mu_{f}}^{T} \mathbb{Y}_{\Lambda_{f}, n_{f}} R_{\Lambda_{f}, n_{f}} \mathbb{H}_{\Lambda_{f}, n_{f} ; \Lambda \mu}\right)\left(\mathbb{H}_{\Lambda_{f}, n_{f} ; \Lambda_{\mu}}^{T} R_{\Lambda_{f}, n_{f}} \mathbb{Y}_{\Lambda_{f}, n_{f}}^{\dagger} \mathbb{C}_{\Lambda_{f} \mu_{f}}^{*}\right)}{\mathbb{C}_{\Lambda_{f} \mu_{f}}^{T} \mathbb{Y}_{\Lambda_{f}, n_{f}} R_{\Lambda_{f}, n_{f}} \mathbb{Y}_{\Lambda_{f}, n_{f}}^{\dagger} \mathbb{C}_{\Lambda_{f} \mu_{f}}^{*}}}
\end{aligned}
$$

where we have used Eqs. $4.9 \& 4.58$ to write the second equality. It is important to emphasize the dependence that $\mathbb{C}^{T} \mathbb{Y}$ has on the two-body interpolators used, and it is essential to use the same interpolators in the two-point and three-point functions for the second equality to follow. Indeed, although we constructed our two-body interpolators from scalar fields (with residue one at the mass pole), this result holds for any interpolating field with the desired quantum numbers. Any nontrivial overlap factors cancel between numerator and denominator. 


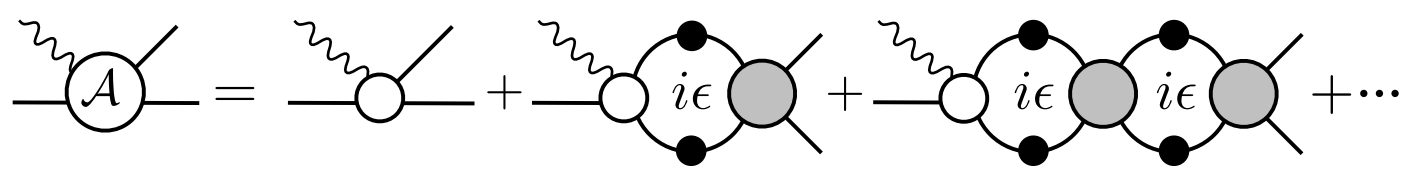

(a)

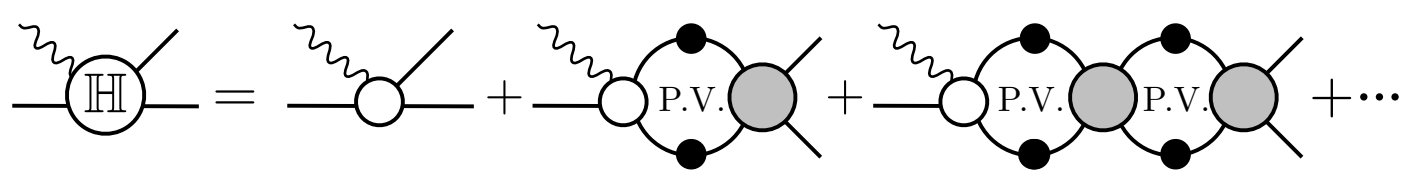

(b)

Figure 4.4: In order to illustrate the differences and similarities between the transition amplitudes a) $\mathcal{A}$ and b) $\mathbb{H}$, we show their diagrammatically representation for the singlechannel case in terms of the LO transition amplitudes (defined in Fig. 4.3(b)), kernels (defined in Fig. 4.1(b) and infinite volume loops. The infinite volume loops of $\mathcal{A}$ are evaluated using the $i \epsilon$ prescription, while those of $\mathbb{H}$ are evaluated using the principal value, as explicitly denoted. For multichannel scenarios one simply upgrades the kernels and two-particle loops to be matrices in the number of open channels and the LO transition amplitude becomes a vector in the space as depicted in Fig. 4.3. Single particle propagators are fully dressed as defined in Fig. 4.1(c), 
For multichannel systems, one needs to evaluate the three-point correlation function using a current that couples to all open channels, as defined in Eq. 4.82, In this case one has the freedom to choose which flavor of two-particle operator is used in evaluating the correlation function. We define

$$
\begin{aligned}
& C_{\Lambda_{f} \mu_{f}, a ; \Lambda \mu}^{(1 \rightarrow 2)}\left(x_{f, 0}-y_{0} ; y_{0}-x_{i, 0}\right)=\left\langle 0\left|\mathcal{O}_{\Lambda_{f} \mu_{f}, a}\left(x_{f, 0}, \mathbf{P}_{f}\right) \widetilde{\mathcal{J}}_{\Lambda \mu}^{[J, P,|\lambda|]}\left(y_{0}, \mathbf{Q}\right) \varphi^{\dagger}\left(x_{i, 0},-\mathbf{P}_{i}\right)\right| 0\right\rangle \\
& =\sum_{n_{f}} e^{-E_{\Lambda_{f}, n_{f}}\left(x_{f, 0}-y_{0}\right)} e^{-E_{\Lambda_{i}, 0}\left(y_{0}-x_{i, 0}\right)}\left\langle 0\left|\mathcal{O}_{\Lambda \mu, a}\left(0, \mathbf{P}_{f}\right)\right| E_{\Lambda_{f}, n_{f}} \mathbf{P}_{f} ; L\right\rangle \\
& \quad \times\left\langle E_{\Lambda_{f}, n_{f}} \mathbf{P}_{f} ; L\left|\widetilde{\mathcal{J}}_{\Lambda \mu}^{[J, P,|\lambda|]}(0, \mathbf{Q})\right| E_{\Lambda_{i}, 0} \mathbf{P}_{i} ; L\right\rangle\left\langle E_{\Lambda_{i}, 0} \mathbf{P}_{i} ; L\left|\varphi^{\dagger}\left(0,-\mathbf{P}_{i}\right)\right| 0\right\rangle
\end{aligned}
$$

This generic representation of the three-point function is diagrammatically depicted in Fig. 4.3(a), Following the steps above, it is straightforward to see that Eq. 4.92 generalizes to

$$
\begin{aligned}
& \left|\left\langle E_{\Lambda_{f}, n_{f}} \mathbf{P}_{f} ; L\left|\widetilde{\mathcal{J}}_{\Lambda \mu}^{[J, P,|\lambda|]}\left(0, \mathbf{P}_{f}-\mathbf{P}_{i}\right)\right| E_{\Lambda_{i}, 0} \mathbf{P}_{i} ; L\right\rangle\right|= \\
& \frac{1}{\sqrt{2 E_{\Lambda_{i}, 0}}} \sqrt{\frac{\left(\mathbb{C}_{\Lambda_{f} \mu_{f}}^{T}\left[\mathbb{Y}_{\Lambda_{f}, n_{f}} R_{\Lambda_{f}, n_{f}} \mathbb{H}_{\Lambda_{f}, n_{f} ; \Lambda \mu}\right]_{a}\right)\left(\left[\mathbb{H}_{\Lambda_{f}, n_{f} ; \Lambda_{\mu}}^{T} R_{\Lambda_{f}, n_{f}} \mathbb{Y}_{\Lambda_{f}, n_{f}}^{\dagger}\right]_{a} \mathbb{C}_{\Lambda_{f} \mu_{f}}^{*}\right)}{\mathbb{C}_{\Lambda_{f} \mu_{f}}^{T}\left[\mathbb{Y}_{\Lambda_{f}, n_{f}} R_{\Lambda_{f}, n_{f}} \mathbb{Y}_{\Lambda_{f}, n_{f}}^{\dagger}\right]_{a a} \mathbb{C}_{\Lambda_{f} \mu_{f}}^{*}}}
\end{aligned}
$$

where the repeated channel indices on the right-hand side are not summed.

We now show that this result is equivalent to the main result of this work, Eq. 4.2 above. To do so we define

$$
\mathbb{V}_{b}^{(a)} \equiv \mathbb{Y}_{\Lambda_{f}, n_{f} ; a, b}^{\dagger} \mathbb{C}_{\Lambda_{f} \mu_{f}}^{*}
$$

where $a$ and $b$ are channel indices. We stress that, for each fixed value of $a, \mathbb{V}_{b}^{(a)}$ is a column in angular-momentum/channel space. Suppressing the channel index, $b$, this notation allows us to rewrite Eq. 4.94 as

$$
\begin{aligned}
&\left|\left\langle E_{\Lambda_{f}, n_{f}} ; L\left|\tilde{\mathcal{J}}_{\Lambda \mu}^{[J, P,|\lambda|]}\right| E_{\Lambda_{i}, 0} ; L\right\rangle\right|= \\
& \frac{1}{\sqrt{2 E_{\Lambda_{i}, 0}}} \sqrt{\frac{\left[\mathbb{V}^{(a) \dagger} R_{\Lambda_{f}, n_{f}} \mathbb{H}_{\Lambda_{f}, n_{f} ; \Lambda \mu}\right]\left[\mathbb{H}_{\Lambda_{f}, n_{f} ; \Lambda \mu}^{T} R_{\Lambda_{f}, n_{f}} \mathbb{V}^{(a)}\right]}{\left[\mathbb{V}^{(a) \dagger} R_{\Lambda_{f}, n_{f}} \mathbb{V}^{(a)}\right]}}
\end{aligned}
$$

Here we have dropped all momentum and time labels for compactness of notation. 
We next observe that $R_{\Lambda_{f}, n_{f}}$, which is Hermitian and therefore diagonalizable, has only one non-zero eigenvalue. To see this, recall that $R_{\Lambda_{f}, n_{f}}$ is equal to a scalar prefactor times $\operatorname{adj}\left[\mathbb{M}\left(P_{0, M}=E_{\Lambda, n}\right)\right]_{\Lambda_{f}}$. The adjugate here is understood as a matrix in angularmomentum/channel space, that has been projected onto the $\Lambda_{f}$ subspace. We now consider the adjugate as a function of $\epsilon_{n} \equiv P_{0, M}-E_{\Lambda, n}$, and show that all but one of its eigenvalues vanishes as $\epsilon_{n} \rightarrow 0$. Recall the defining relation

$$
\operatorname{adj}\left[\mathbb{M}\left(\epsilon_{n}\right)\right]=\operatorname{det}\left[\mathbb{M}\left(\epsilon_{n}\right)\right]\left[\mathbb{M}\left(\epsilon_{n}\right)\right]^{-1}
$$

Formally diagonalizing both sides, we argue that exactly one of the eigenvalues of $\left[\mathbb{M}\left(\epsilon_{n}\right)\right]^{-1}$ scales as $1 / \epsilon_{n}$ and the rest are finite.

Note that the divergence of two eigenvalues, which we discount, would imply the existence two orthogonal states that are exactly degenerate in finite volume. This represents two possibilities. The first is that distinct energies coincide only at certain values of $L$. This would imply a level crossing, which does not occur unless the Hilbert space divides into distinct, non-interacting subspaces. The second possibility is that the finite volume spectrum includes states that are degenerate for all values of $L$. This occurs whenever there is a symmetry relating the finite-volume states. However, in the present context the matrix has been projected to a particular irrep and row. It follows that, within the subspace that we consider, exactly one of the eigenvalues of $\left[\mathbb{M}\left(\epsilon_{n}\right)\right]^{-1}$ scales as $1 / \epsilon_{n}$. This in turn implies that the determinant of $\mathbb{M}\left(\epsilon_{n}\right)$ vanishes as $\epsilon_{n}$ or faster, and thus all but one of the adjugate's eigenvalues vanishes.

We denote the nonzero eigenvalue of $R_{\Lambda_{f}, \mu_{f}}$ by $\lambda$ and the corresponding eigenvector, $\mathbb{E}$. We also introduce $\mathbb{E}_{1}, \mathbb{E}_{2}, \cdots$ as the remaining orthonormal set that is annihilated by $R_{\Lambda_{f}, n_{f}}$. These eigenvectors span the space, so we may substitute $\mathbb{V}^{(a)}=c \mathbb{E}+\sum c_{i} \mathbb{E}_{i}$ and deduce

$$
\begin{aligned}
\left|\left\langle E_{\Lambda_{f}, n_{f}} ; L\left|\widetilde{\mathcal{J}}_{\Lambda \mu}^{[J, P,|\lambda|]}\right| E_{\Lambda_{i}, 0} ; L\right\rangle\right| & =\frac{1}{\sqrt{2 E_{\Lambda_{i}, 0}}} \sqrt{\frac{\left[c^{*} \lambda \mathbb{E}^{\dagger} \mathbb{H}_{\Lambda_{f}, n_{f} ; \Lambda \mu}\right]\left[\mathbb{H}_{\Lambda_{f}, n_{f} ; \Lambda \mu}^{T} c \lambda \mathbb{E}\right]}{\lambda c^{*} \mathbb{E}^{\dagger} \mathbb{E} c}}, \\
& =\frac{1}{\sqrt{2 E_{\Lambda_{i}, 0}}} \sqrt{\operatorname{Tr}\left[\lambda \mathbb{E}^{\dagger} \mathbb{H}_{\Lambda_{f}, n_{f} ; \Lambda \mu} \mathbb{H}_{\Lambda_{f}, n_{f} ; \Lambda \mu}^{T} \mathbb{E}\right]} \\
& =\frac{1}{\sqrt{2 E_{\Lambda_{i}, 0}}} \sqrt{\operatorname{Tr}\left[\mathbb{H}_{\Lambda_{f}, n_{f} ; \Lambda \mu}^{T} \lambda \mathbb{E}^{\dagger} \mathbb{H}_{\Lambda_{f}, n_{f} ; \Lambda \mu}\right]}
\end{aligned}
$$


where in the first line we acted $R_{\Lambda_{f}, n_{f}}$ on each eigenvector, in the second line we canceled common factors and inserted a redundant trace, and in the third we used the cyclic property of the trace. Observing finally that

$$
R_{\Lambda_{f}, n_{f}}=\lambda \mathbb{E}^{\dagger}
$$

we conclude

$$
\begin{aligned}
&\left|\left\langle E_{\Lambda_{f}, n_{f}} \mathbf{P}_{f} ; L\left|\tilde{\mathcal{J}}_{\Lambda \mu}^{[J, P,|\lambda|]}\left(0, \mathbf{P}_{f}-\mathbf{P}_{i}\right)\right| E_{\Lambda_{i}, 0} \mathbf{P}_{i} ; L\right\rangle\right|= \\
& \frac{1}{\sqrt{2 E_{\Lambda_{i}, 0}}} \sqrt{\left[\mathbb{H}_{\Lambda_{f}, n_{f} ; \Lambda \mu}^{T} R_{\Lambda_{f}, n_{f}} \mathbb{H}_{\Lambda_{f}, n_{f} ; \Lambda \mu}\right]} .
\end{aligned}
$$

\subsubsection{Relation of $\mathbb{H}$ to infinite-volume matrix elements}

In this section we relate $\mathbb{H}_{\Lambda_{f}, n_{f} ; \Lambda \mu ; J m_{J}}=\mathbb{H}_{\Lambda_{f} ; \Lambda \mu ; J m_{J}}\left(E_{\Lambda_{f}, n_{f}}^{*}\right)$ to infinite-volume matrix elements. Here we have given the full set of indices including $J m_{J}=l m$, which was suppressed in the steps above. We have also emphasized that the label $n_{f}$ only refers to the particular two-particle pole at which the transition amplitude is evaluated. Finally, we stress that the subscript $\Lambda_{f}$ on $\mathbb{H}$ indicates that the angular momentum space has been projected onto a finite-volume irrep. For example in the case of $\Lambda_{f}=\mathbb{A}_{1}^{+}$the transition amplitude will include $J=0, J=4$ and certain higher waves, but not $J=2, J=3$. However by considering different irreps one can in principal sample all partial waves, and so construct an unprojected vector $\mathbb{H}_{\Lambda \mu ; J m_{J}}$.

To give the relation to physical matrix elements, we first connect this transition amplitude, defined using principal-value prescription, to the amplitude defined via $i \epsilon$ prescription. We label the latter $\mathcal{A}_{\Lambda \mu ; J m_{J}}$. Both amplitudes are explicitly shown in Fig. B.1 and the relationship between the two is found by noting that the difference in each two-particle loop is a simple kinematic factor, determined by the residue of the propagators at the poles. This is very similar to the relation between $\mathbb{K}$ and $\mathcal{M}$ discussed above. We find

$$
\begin{aligned}
\mathcal{A} & =\mathbb{H}+\mathbb{K}\left(i \mathbb{P}^{2} / 2\right) \mathbb{H}+\mathbb{K}\left(i \mathbb{P}^{2} / 2\right) \mathbb{K}\left(i \mathbb{P}^{2} / 2\right) \mathbb{H}+\cdots=\left[\frac{1}{1-\mathbb{K}\left(i \mathbb{P}^{2} / 2\right)}\right] \mathbb{H} \\
& =\left[\frac{1}{\mathbb{K}^{-1}-\left(i \mathbb{P}^{2} / 2\right)}\right] \mathbb{K}^{-1} \mathbb{H}=\mathcal{M} \mathbb{K}^{-1} \mathbb{H} .
\end{aligned}
$$


For systems where there is only a single channel present these are just scalars, otherwise these are matrices in the space of open channels. Note that $\mathbb{H}$ is pure real and thus the phases of $\mathcal{A}$ are determined entirely by the strong interaction, as encoded in $\mathbb{K}^{-1} \mathcal{M}$. In the single channel case we see that the phase of $\mathcal{A}$ is equal to the elastic scattering phase of the two-particle channel considered. Thus Eq. 4.103 is simply the generalization of Watson's theorem for multichannel systems. This relation motivates the definition

$$
\mathcal{R}_{\Lambda_{f}, n_{f}}=\left[\mathcal{M}^{-1 \dagger} \mathbb{K} R \mathbb{K} \mathcal{M}^{-1}\right]_{\Lambda_{f}, n_{f}}
$$

which allows us to compactly display our main result in terms of $\mathcal{A}$, as in Eq. 4.2 above.

$\mathcal{A}_{\Lambda \mu ; J m_{J}}$ is trivially related to the infinite-volume matrix element of the current. To see this, we first rewrite the current $\widetilde{\mathcal{J}}_{\Lambda \mu}^{[J, P,|\lambda|]}\left(x_{0}, \mathbf{Q}\right)$, Eq. 4.82 in infinite volume and set $x_{0}=0$,

$$
\begin{aligned}
\widetilde{\mathcal{J}}_{\Lambda \mu}^{[J, P,|\lambda|]}(0, \mathbf{Q} ; \infty)=\sum_{a}^{N} \xi_{a} & \int \frac{d^{4} P_{f}}{(2 \pi)^{4}} \frac{d^{4} P_{i}}{(2 \pi)^{4}} \frac{d^{4} k}{(2 \pi)^{4}} \bar{\varphi}_{a}^{\dagger}\left(-P_{f}+k\right) \widetilde{\varphi}_{a}^{\dagger}(-k) \varphi\left(P_{i}\right) \\
& \times h_{\Lambda \mu}^{[J, P,|\lambda|]}\left(P_{i}, P_{f}-k, k, a\right)(2 \pi)^{3} \delta^{3}\left(\mathbf{P}_{f}-\mathbf{P}_{i}-\mathbf{Q}\right) .
\end{aligned}
$$

Note that we still label the current by $\Lambda \mu$. The linear combinations that relate this basis to more standard infinite-volume bases are discussed above and are perfectly well defined, even though the finite-volume symmetry group does not play a role at this stage. Requiring only that states are normalized according to the standard infinite-volume relativistic convention (Eq. 4.8) and also that the single-particle operators have propagators with unit residue (Eq. 4.7) one arrives at Eq. 4.3.

\subsubsection{Examples of applications of Eq. 4.2}

$$
K \rightarrow \pi \pi \text { decay amplitude }
$$

First, we demonstrate that this formalism properly recovers the well known result for $K \rightarrow$ $\pi \pi$ weak decay. In this case, the initial state is a single kaon and the external current is a pseudoscalar. The current cannot inject any momentum, so we set $\mathbf{P}_{f}=\mathbf{P}_{i}$. By conservation of angular momentum, the infinite-volume current can only create a two-pion state in an S-wave. For a finite-volume system we restrict ourselves to irreps that have strong overlap with S-wave and we neglect higher partial wave contributions. Within this 
approximation, our master equation gives the following relationship between the infinitevolume transition amplitude and the finite-volume matrix element

$\frac{\left|\mathcal{A}_{S, n_{f}}\right|^{2}}{\left|\left\langle\pi \pi, E_{n_{f}} \mathbf{P}, \Lambda_{f} \mu_{f} ; L\left|\widetilde{\mathcal{J}}_{\Lambda \mu}^{[0,-1,|0|]}(0, \mathbf{0})\right| K, E_{K} \mathbf{P} ; L\right\rangle\right|^{2}}=\left.\frac{16 \pi E_{i} E_{n_{f}}^{*}}{q_{n_{f}}^{*} \xi} \frac{\partial\left(\delta_{S}+\phi_{00}^{\mathbf{d}}\right)}{\partial P_{0, M}}\right|_{P_{0, M}=E_{n_{f}}}$

For the problem at hand $E_{i}$ is equal to the energy of the incoming kaon and the symmetry factor $\xi$ is equal to $1 / 2$. If one wishes, it is straight forward to replace the derivative with respect to total energy with a derivative with respect to relative momentum. Doing so, one finds agreement with Refs. [70, 130, 131, 190] in the limit that the initial and final state are exactly degenerate. Note that, since the current is evaluated at a specific time slice, the current need not conserve energy and this result reflects that fact. For processes such as $K \rightarrow \pi \pi$ this is an artifact, and one would want to assure to extract a $\pi \pi$ energy level that is in the vicinity of the kaon energy.

$\pi \gamma \rightarrow \pi \pi$ form factor

Unlike the previous example, for a process such as $\pi \gamma \rightarrow \pi \pi$ the external current can inject arbitrary momentum. For such a process, the lowest energy configuration of the final state is a P-wave. Therefore, it is expected that the Lellouch-Lüscher factor gets modified. Since the two particles in the final state are degenerate, odd and even partial waves cannot mix. By ignoring contamination from the F-wave and using the results of Section 4.1.2 one finds the generalization of the previous result for two particles in a $\mathrm{P}$-wave,

$$
\begin{gathered}
\frac{\left|\mathcal{A}_{\Lambda_{f} \mu_{f}, n_{f} ; \Lambda \mu ; J=1}\right|^{2}}{\left|\left\langle\pi \pi, E_{n_{f}} \mathbf{P}_{f}, \Lambda_{f} \mu_{f} ; L\left|\widetilde{\mathcal{J}}_{\Lambda \mu}^{[1,-1,|\lambda|]}\left(0, \mathbf{P}_{f}-\mathbf{P}_{i}\right)\right| \pi, E_{i} \mathbf{P}_{i} ; L\right\rangle\right|^{2}}=16 \pi E_{i} \frac{E_{n_{f}}}{q_{n_{f}}^{*} \xi} \sin ^{2} \delta_{P} \\
\times\left.\left[\csc ^{2} \delta_{P} \frac{\partial \delta_{P}}{\partial P_{0, M}}+\csc ^{2} \phi_{00}^{\mathbf{d}} \frac{\partial \phi_{00}^{\mathbf{d}}}{\partial P_{0, M}}+\sum_{m=0,2} \alpha_{2 m, \Lambda_{f}} \csc ^{2} \phi_{2 m}^{\mathbf{d}} \frac{\partial \phi_{2 m}^{\mathbf{d}}}{\partial P_{0, M}}\right]\right|_{P_{0, M}=E_{n_{f}}}
\end{gathered}
$$

where $\alpha_{2 m, T_{1}^{-}}=0$ and the values of $\alpha_{2 m, \Lambda_{f}}$ for other relevant irreps are given in Table 4.2 . The $J=1$ superscript on the transition amplitude means that we have integrated it against one of the $l=1$ spherical harmonics. As discussed above, this projection is performed in the two-particle center of mass frame. Again one may set $\xi=1 / 2$ for this case, since the two outgoing particles are identical. Observe that the right-hand side does not depend on the representation of the current or the single-particle state. 
The right-hand side effectively corrects for the large finite-volume artifacts associated with the two-particle state. This gives a one-to-one mapping between the finite-volume and infinite-volume form factors for this process. The result thus allows one to determine, using LQCD, the same quantity that is extracted from experiments. If one wants to evaluate this form factor at the $\rho$ pole, in order to study processes such as $\pi \gamma \rightarrow \rho$, then it is necessary to analytically continue into the complex plane [192]. This requires parameterizing the form factor as function of the exchange momentum as well as the relative momentum between the two pions in the P-wave. By fitting this function to the LQCD results, one can study the behavior of the form factor as a function of the exchange momentum at the resonance pole.

\section{Two-dimensional case}

As we have already stressed before, partial wave mixing is inevitable when performing calculations in a finite volume, and Eq. 4.2 reflects this reduction of rotational symmetry. In addition, the final two-particle state may in general have overlap with more than one infinite-volume state. This leads us to consider a generic scenario where the matrix $\mathcal{R}$ in Eq. 4.2 is two dimensional. In order to avoid introducing additional notation we consider the form of the main result using infinite-volume quantities that are defined via principal-value prescription, namely Eq. 4.102.

In Section 4.1 .3 we discussed one explicit example for a $\pi K$ boosted state, where we neglected contributions from $J \geq 2$ partial waves. We could also consider a system with two open channels where we ignore partial wave mixing, e.g., $\pi \pi-K \bar{K}$. In the first case, the finite volume matrix $\mathbb{F}_{\Lambda_{f}}^{V}$ will have off-diagonal terms but the K-matrix will be diagonal. In the second case this is reversed; the K-matrix has non-zero off diagonal terms while $\mathbb{F}_{\Lambda_{f}}^{V}$ is diagonal. In order to accommodate these two scenarios simultaneously, we allow the Kmatrix and the $\mathbb{F}_{\Lambda_{f}}^{V}$ matrix to have off diagonal terms. The spectrum of this system must satisfy

$$
\operatorname{det}\left[\mathbb{M}_{\Lambda_{f}}\right]=\operatorname{det}\left[\mathbb{K}_{\Lambda_{f}}+\left(\mathbb{F}_{\Lambda_{f}}^{V}\right)^{-1}\right]=0
$$

Requiring the determinant of $\mathbb{M}_{\Lambda_{f}}$ to vanish, implies that we can generically write its 
adjugate in the following form,

$\left.\operatorname{adj}\left[\mathbb{M}_{\Lambda_{\mathrm{f}}}\right]\right|_{\mathrm{P}_{0, \mathrm{M}}=\mathrm{E}_{\Lambda_{\mathrm{f}}, \mathrm{n}_{\mathrm{f}}}}=\left(\begin{array}{ccc}{\left[\mathbb{M}_{\left.\Lambda_{f}\right]_{22}}\right.} & -e^{i \theta_{\Lambda_{f}}} & \sqrt{\left[\mathbb{M}_{\left.\Lambda_{f}\right]_{22}}\left[\mathbb{M}_{\left.\Lambda_{f}\right]_{11}}\right.\right.} \\ -e^{-i \theta_{\Lambda_{f}}} \sqrt{\left[\mathbb{M}_{\left.\Lambda_{f}\right]_{22}\left[\mathbb{M}_{\Lambda_{f}}\right]_{11}}\right.} & {\left[\mathbb{M}_{\Lambda_{f}}\right]_{11}}\end{array}\right)$,

where $\theta_{\Lambda_{f}}$ is an unknown real phase.

Inserting the above expression into Eq. 4.102, one finds

$$
\begin{aligned}
& \left|\left\langle E_{\Lambda_{f}, n_{f}} \mathbf{P}_{f} ; L\left|\tilde{\mathcal{J}}_{\Lambda \mu}^{[J, P,|\lambda|]}\left(0, \mathbf{P}_{f}-\mathbf{P}_{i}\right)\right| E_{\Lambda_{i}, 0} \mathbf{P}_{i} ; L\right\rangle\right|^{2}=\frac{1}{2 E_{\Lambda_{i}, 0}} \\
& \times\left.\left(\frac{[\mathbb{H}]_{1}^{2}\left[\mathbb{M}_{\Lambda_{f}}\right]_{22}+[\mathbb{H}]_{2}^{2}\left[\mathbb{M}_{\Lambda_{f}}\right]_{11}-2[\mathbb{H}]_{1}[\mathbb{H}]_{2} \sqrt{\left[\mathbb{M}_{\Lambda_{f}}\right]_{11}\left[\mathbb{M}_{\Lambda_{f}}\right]_{22}} \cos \left[\theta_{\Lambda_{f}}\right]}{\operatorname{tr}\left[\operatorname{adj}\left[\mathbb{M}_{\Lambda_{f}}\right] \frac{\partial \mathbb{M}_{\Lambda_{f}}}{\partial P_{0, M}}\right]}\right)\right|_{P_{0, M}=E_{\Lambda_{f}, n_{f}}}
\end{aligned}
$$

where the subscripts of $\mathbb{H}_{\Lambda_{f} \mu_{f}, n_{f} ; \Lambda \mu}$ have been suppressed in the last line for compactness. This nontrivial result illustrates the power of Eq. 4.2.

$D \rightarrow\{\pi \pi, K \bar{K}\}$ decays

Assuming sufficiently heavy pion masses where the multi-particle threshold lies above the energy of the $D$ mesons, Eq. 4.110 allows for the studies of $D \rightarrow\{\pi \pi, K \bar{K}\}$ decays. To find the equivalence between the result presented in the previous section and the result presented in Ref. [140], we rederive the result of Ref. [140] using notation presented here. This allows for a more compact representation of the result. In Ref. [140], the authors followed the trick first utilized by Lellouch and Lüscher in the studies of $K \rightarrow \pi \pi$ decays. We present this method in the context of the two-channel system.

The argument proceeds by modifying the $\pi \pi-K \bar{K}$ correlation function, by including a contribution to the Hamiltonian density due to the weak interaction. We denote this perturbative shift by $\lambda \mathcal{H}_{W}(x)$, where $\lambda$ is a free parameter that allows us to organize an expansion. The modified Hamiltonian density allows for $\pi \pi-K \bar{K}$ states and the $D$ meson state to mix, both in a finite and infinite volume. Considering first the finite-volume theory, we tune the box size $L$ such that the $D$ state and some $\pi \pi-K \bar{K}$ finite-volume state are 
exactly degenerate (for a given total momentum). The presence of the weak interaction will break the degeneracy and result in two nearly degenerate states with energies

$$
E^{(1)}=E_{D} \pm \lambda L^{3}\left|\left\langle E_{D} \mathbf{P} ; L\left|\mathcal{H}_{W}(0)\right| D, E_{D} \mathbf{P} ; L\right\rangle\right|,
$$

where we have only kept the leading order contribution in $\lambda$ and where $E_{D}=\sqrt{M_{D}^{2}+\mathbf{P}^{2}}$ with $M_{D}$ the $D$ meson mass. Turning to the infinite-volume theory, the weak perturbation has the effect of modifying the scattering amplitude. This modification is due to the additional interaction that couples the $D$ to the two-particle states. The shift in the scattering amplitude contains two insertions of the weak Hamiltonian, one for transitioning from two particles to the $D$ and one for transitioning back to two particles. Thus the shift is generically $\mathcal{O}\left(\lambda^{2}\right)$, but in the present case we are evaluating the amplitude at an energy which is shifted by $\mathcal{O}(\lambda)$ from $E_{D}$. This enhances the change of the scattering amplitude to be $\mathcal{O}(\lambda)$. Putting these two pieces together one finds [140]

$$
\mathcal{M}^{(1)}=\mathcal{M}^{(0)} \mp \lambda \Delta \mathcal{M}
$$

where

$$
\Delta \mathcal{M}=\frac{1}{2 E_{D} L^{3}\left|\left\langle E_{D} \mathbf{P} ; L\left|\mathcal{H}_{W}(0)\right| D, E_{D} \mathbf{P} ; L\right\rangle\right|}\left(\begin{array}{cc}
\left|\mathcal{A}_{D \rightarrow \pi \pi}\right|^{2} & \mathcal{A}_{D \rightarrow \pi \pi} \mathcal{A}_{D \rightarrow K \bar{K}}^{\dagger} \\
\mathcal{A}_{D \rightarrow \pi \pi}^{\dagger} \mathcal{A}_{D \rightarrow K \bar{K}} & \left|\mathcal{A}_{D \rightarrow K \bar{K}}\right|^{2}
\end{array}\right) .
$$

We next find it convenient to rewrite this perturbation to the scattering amplitude as a perturbation to the K-matrix. To do this, we follow the reasoning of Eq. 4.103 and observe that the only difference between the transition amplitude and the scattering amplitude is that for the latter we need to include the imaginary part of the diagrams associated with both incoming as well as outgoing two particle states. This leads to the following relation between $\Delta \mathcal{M}$ and $\Delta \mathbb{K}$,

$$
\Delta \mathbb{K}=\mathbb{K} \mathcal{M}^{-1} \Delta \mathcal{M} \mathcal{M}^{-1} \mathbb{K}
$$

At this point we can combine the shift in the finite-volume spectrum with the shift in the infinite-volume $\mathrm{K}$-matrix to determine the leading order modification to $\mathbb{M}$, defined in 
Eq. 4.47. We find that the matrix is shifted by an amount

$$
\lambda \Delta \mathbb{M}=\left.\lambda \Delta E \frac{\partial \mathbb{M}}{\partial P_{0, M}}\right|_{P_{0, M}=E_{D}} \mp \lambda \Delta \mathbb{K} .
$$

where $\Delta E=L^{3}\left|\left\langle E_{D} \mathbf{P} ; L\left|\mathcal{H}_{W}(0)\right| D, E_{D} \mathbf{P} ; L\right\rangle\right|$. Of course, the quantization must also be valid for the perturbed theory. We thus deduce that the linear shift to the determinant of $\mathbb{M}$ should vanish

$$
\operatorname{det}[\mathbb{M}(\lambda)]|=\operatorname{det}[\mathbb{M}(0)]+\lambda \operatorname{tr}[\operatorname{adj}[\mathbb{M}(0)] \Delta \mathbb{M}]=\lambda \operatorname{tr}[\operatorname{adj}[\mathbb{M}] \Delta \mathbb{M}]|_{P_{0, M}=E_{D}}=0
$$

where we have used the fact that $\mathbb{M}(0)$ also has vanishing determinant, since this defines the quantization condition of the unperturbed theory.

Showing that this result is equivalent to Eq. 4.110 require some algebra. First we rewrite the relation as

$$
\begin{aligned}
\Delta E & =\left.\frac{\operatorname{tr}[\operatorname{adj}[\mathbb{M}] \Delta \mathbb{K}]}{\operatorname{tr}\left[\operatorname{adj}\left[\mathbb{M} \frac{\partial \mathbb{M}}{\partial P_{0, M}}\right]\right.}\right|_{P_{0, M}=E_{D}} \\
& =\left.\frac{1}{2 E_{D}}\left(\frac{\left|[\mathbb{H}]_{1} \sqrt{[\mathbb{M}]_{22}}-[\mathbb{H}]_{2} \sqrt{[\mathbb{M}]_{11}}\right|^{2}}{\operatorname{tr}\left[\operatorname{adj}[\mathbb{M}] \frac{\partial \mathbb{M}}{\partial P_{0, M}}\right] L^{3}\left|\left\langle E_{D} \mathbf{P} ; L\left|\mathcal{H}_{W}(0)\right| D, E_{D} \mathbf{P} ; L\right\rangle\right|}\right)\right|_{P_{0, M}=E_{D}},
\end{aligned}
$$

where we have used Eq. 4.103, $4.109 \& 4.114$ in finding the second equality above. Finally, using Eq. 4.111, one obtains

$$
\begin{aligned}
& L^{6}\left|\left\langle E_{D} \mathbf{P} ; L\left|\mathcal{H}_{W}(0)\right| D, E_{D} \mathbf{P} ; L\right\rangle\right|^{2} \\
& \qquad \quad=\left.\frac{1}{2 E_{D}}\left(\frac{\left|[\mathbb{H}]_{1} \sqrt{[\mathbb{M}]_{22}}-[\mathbb{H}]_{2} \sqrt{[\mathbb{M}]_{11}}\right|^{2}}{\operatorname{tr}\left[\operatorname{adj}[\mathbb{M}] \frac{\partial \mathbb{M}}{\partial P_{0, M}}\right]}\right)\right|_{P_{0, M}=E_{D}} .
\end{aligned}
$$

This is equivalent to Eq. 4.110 for the special case where the initial and final states are exactly degenerate, have the same total momentum and $\theta_{\Lambda_{f}}=0$. Note that the left hand side of the above equation contains an extra factor of $L^{6}$, this is because the current in Eq. 4.110 is in momentum-space.

In deriving this result following Lellouch and Lüscher trick one is forced to consider a scenario where the final two-particle state is degenerate with the heavy single particle state. 
For $D \rightarrow\{\pi \pi, K \bar{K}\}$ decays, this would make the $\pi \pi$ highly relativistic with an overall energy that is above the four-particle threshold, unless one considers very heavy pion masses or unphysically light charm quarks. In deriving Eq. 4.110 we made no assumptions of the sort and as a result the initial and final states can have arbitrarily different energies. This of course would mean that $D \rightarrow\{\pi \pi, K \bar{K}\}$ one would necessarily evaluate form factors with large exchanged energies, but ultimately one would need to extrapolate to the zero exchanged momenta limit to recover the physical process of interest.

$B \rightarrow \pi K$ form factors

One example where partial wave mixing may in general not be small is in the studies of $B \rightarrow \pi K$ form factors. This is due to the fact that for boosted systems the final state will be an admixture of even and odd partial waves. In particular, if interested in case where the infinite volume final state has overlap with the $K^{*}(892)$ resonance, we must consider irreps that have strong overlap with the $\pi K \mathrm{P}$-wave. If the final state is at rest or if it is in the $\mathbb{E}$ irrep for $\mathbf{d}=(00 n), \mathbb{B}_{1}$ and $\mathbb{B}_{2}$ for $\mathbf{d}=(n n 0)$, or $\mathbb{E}$ for $\mathbf{d}=(n n n)$, and if we neglect the contribution from the $\mathrm{D}$ and higher partial waves by following the discussion of Section 4.1 .3 one finds that the ratio of the infinite and finite volume form factors for vector or pseudo vector currents satisfies Eq. 4.107 where the symmetry factor $\xi$ must be set equal to 1 .

For the $\mathbb{A}_{1}$ irrep of the $\mathrm{Dic}_{4}$ group, one simply needs to insert the expressions for the on-shell K-matrix in Eq. 4.65 along with $\mathbb{F}_{\mathbb{A}_{1}}$ in Eq. 4.67 onto Eq. 4.110 to find the relation between the finite volume and infinite volume form factors. Because of the symmetries of the infinite volume only one of the transition amplitudes is non vanishing. For example, if we consider the case where the current is a current subdued from $J=1$ with odd parity, then $\mathbb{H}_{S, n_{f} ; \Lambda \mu}$ must exactly vanish. Therefore for vector currents Eq. 4.110 simplifies down 
to

$$
\begin{gathered}
\frac{\left|\mathcal{A}_{P 0, n_{f} ; \Lambda \mu}\right|^{2}}{\left|\left\langle\pi K, E_{n_{f}} \mathbf{P}_{f}, \Lambda_{f} \mu_{f} ; L\left|\widetilde{\mathcal{J}}_{\Lambda \mu}^{[1,-1,|\lambda|]}(0, \mathbf{Q})\right| B^{0}, E_{B^{0}} \mathbf{P}_{i} ; L\right\rangle\right|^{2}}= \\
2 E_{\Lambda_{i}, 0} \cos ^{2} \delta_{P}\left|\frac{\operatorname{tr}\left[\operatorname{adj}\left[\mathbb{M}_{\Lambda_{f}}\right] \frac{\partial \mathbb{M}_{\Lambda_{f}}}{\partial P_{0, M}}\right]}{\left[\mathbb{M}_{\Lambda_{f}}\right]_{11}}\right|_{P_{0, M}=E_{\Lambda_{f}, n_{f}}}
\end{gathered}
$$

where $\mathcal{A}_{P 0, n_{f} ; \Lambda \mu}$ denotes the $\mathrm{P}$-wave transition amplitude with zero helicity. This follows from the helicity decomposition of the $\mathbb{A}_{1}$ irrep of the Dic 4 group as shown in Table 4.1(b). For a pseudo vector current or for rank two tensor currents neither $\mathbb{H}_{S, n_{f} ; \Lambda \mu}$ nor $\mathbb{H}_{P m, n_{f} ; \Lambda \mu}$ need vanish. Therefore one necessarily must use Eq. 4.110. For the $\mathbb{A}_{1}$ irreps of the $\mathrm{Dic}_{2}$ group one must input the finite volume function and scattering matrices defined in Section 4.1.3 onto the general result for the matrix element of the currents, Eq. 4.102.

As discussed in the previous section, this result does not require that the initial and final state are exactly degenerate. For studies of B meson decays on the lattice, allowing the initial and final states to be non-degenerate is a necessity, since our formalism does not support multi (more than two) particle states. This result is thus of significance for studies of B meson decays with large energy exchange, such that the final state is below all multi particle thresholds.

Finally, it is important to remember that if interested in studying form factors involving the isospin-1/2 $K \pi$ in the final state, one necessarily must consider the admixture of this with $K \eta$. Although the inelasticity is seen to be small at physical values, this will depend on the quark masses used to perform the calculation. Furthermore, for unphysically large quark masses, such as those in used in Refs. [233, 234, the $K \eta$ threshold is significantly closer to the $K \pi$ threshold than it is in nature. In order to include this mixing between the channels one will have to use Eq. 4.110 when there are two open channels with negligible partial wave mixing or in general Eq. 4.2. 


\section{Chapter 5}

\section{THREE-PARTICLE SCATTERING}

In the last few years, lattice QCD calculations of the properties of resonances have become widespread $\perp^{1}$ Most use a method first proposed by Lüscher in Refs. [127, 128, 142, in which the finite-volume spectrum (obtained using lattice simulations) can be related to infinite-volume scattering amplitudes. This method initially applied to two-particle systems below the inelastic threshold, but has since been extended to systems with multiple two-particle channels [133, 134, 135, 140, 146]. A striking example of the practical implementation of this multi-channel formalism is the recent lattice study of the properties of kaon resonances [92].

Lattice calculations can now routinely determine many spectral levels for a given set of total quantum numbers, and can do so for quark masses approaching physical values. This means that channels involving three or more particles are opening up and must be incorporated into the formalism. Examples include $\omega \rightarrow 3 \pi, K^{*} \rightarrow K \pi \pi$, and $N^{*} \rightarrow N \pi \pi$. Indeed, the study of Ref. [92], although using an unphysically heavy pion mass of $390 \mathrm{MeV}$, was limited by the opening of the $K \pi \pi$ channel. Thus there is strong motivation to extend the finite-volume formalism to include three (or more) particles.

First steps in this direction have been taken in Refs. [138] and [139]. The former work considers the problem in a non-relativistic context, and shows that the finite-volume spectrum is determined (via integral equations) by infinite-volume scattering amplitudes. The latter work reaches the same conclusion in the case in which pairs of particles interact only in the s-wave. Related problems have also been considered in Refs. 238] and [239]. We attempt here to go beyond these works by considering a relativistic theory in which we make no approximation concerning the nature of the two-particle interactions.

Our approach is a generalization of the diagrammatic, field-theoretic approach intro-

\footnotetext{
${ }^{1}$ For recent reviews see Refs. 235, 236, 237].
} 
duced for two particles in Ref. [130]. The finite-volume spectrum is determined by the poles in an appropriate finite-volume correlation function. The method consists of rewriting this correlation function, diagram by diagram, in terms of infinite-volume contributions and kinematic functions which depend on the volume. Summing all diagrams then leads to the desired quantization condition. This approach is straightforward in the two-particle case, but several complications arise with three particles. In the end, however, we are able to obtain a simple-looking quantization condition [Eq. (5.18)], which succeeds in separating finite-volume dependence into kinematical functions.

As in the two-particle quantization conditions, our result is formal in that it involves a determinant over a infinite-dimensional space. Practical applications require truncation of this space. It turns out that such a truncation can be justified for three particles by a simple extension of the arguments used for two particles.

The main drawback of our result is that it depends on a non-standard infinite-volume three-to-three scattering quantity, a modified three-particle K-matrix. The relation of this quantity to physical scattering amplitudes is as yet unclear, but it is under active investigation. Nevertheless, given the results of Refs. [138, 139] in the non-relativistic context, we think it very likely that such a relation exists. In light of this, and given the complicated and lengthy nature of the derivation of our result, we think it appropriate to present this derivation in a separate article. The remainder of this article is organized as follows. We begin, in Sec. 5.1. by presenting our main result. This in itself requires a fairly lengthy introduction and explanation of notation. We next, in Sec. 5.2, describe briefly how the result might be used in practice. The core of the paper is Sec. 5.3, in which we derive our main result. We conclude and discuss the future outlook in Sec. 5.4.

We include three appendices for technical details. Appendix D, derives the key sumintegral difference identity used throughout the derivation Appendix E describes the properties of the modified principal-value pole prescription that we use. Finally, Appendix F discusses in detail an example of using our quantization condition in the isotropic approximation.

A sketch of the result has been given previously in Ref. [168], although some of the technical remarks in that work are incorrect and have been corrected here. 


\subsection{Quantization Condition}

In this section we present the three-particle quantization condition. To explain the result requires some preliminary discussion, particularly about the three-particle scattering amplitude. It also requires the introduction of some rather involved notation. We have attempted to make this section self-contained so that the reader can skip the subsequent lengthy derivation if desired.

Lattice calculations can determine the spectrum of QCD in finite spatial volumes. We assume here a cubic spatial volume of extent $L$ with fields satisfying periodic boundary conditions. We take $L$ large enough to allow neglect of exponentially suppressed corrections of the form $e^{-m L}$, where $m$ is the particle mass. We assume that discretization errors are small and can be ignored, and so work throughout with continuum field theory (zero lattice spacing).

We work in general in a "moving frame". That is, we consider states with non-zero total three-momentum $\vec{P}$. This three momentum is constrained by the boundary conditions to satisfy $\vec{P}=2 \pi \vec{n}_{P} / L$, with $\vec{n}_{P}$ a vector of integers. The total moving-frame energy is denoted $E$, while $E^{*}$ is the energy in the center of mass $(\mathrm{CM})$ frame: $E^{* 2}=E^{2}-\vec{P}^{2}$. (The superscript $*$ is used throughout this work to indicate a quantity boosted to an appropriate

CM frame.) The goal of this section is, at fixed fixed $\{L, \vec{P}\}$, to determine the spectrum of the finite-volume system in terms of infinite-volume scattering amplitudes.

We choose a simple theory for this study: a single real scalar field $\phi$ describing particles of physical mass $m$. Thus all results in this work hold for identical particles. For simplicity, we assume the Lagrangian has a $\mathbb{Z}_{2}$ symmetry that prevents vertices having an odd number of particles. (For pions in QCD this is G-parity.) We otherwise include all vertices, with any even number of fields, and make no assumptions about relative coupling strengths.

Given the $\mathbb{Z}_{2}$ symmetry, the Hilbert space splits into even- and odd-particle states. We are interested here in the latter, which are those created from the vacuum by the field $\phi$ (or by $\phi^{3}, \phi^{5}$, etc.). The spectrum in this sector consists of an isolated single-particle state with $E^{*}=m$, followed by a tower of states that lie close (for large $L$ ) to the energies of three free particles in the finite volume. Such states begin at $E^{*} \approx 3 m$, and it is these 
that we focus on. Their energies typically are shifted from those of three free particles by a difference $\Delta E$ which scales as an inverse power of $L$. Once $E^{*}$ reaches $5 m$, one also has states which lie close to the energies of five free finite-volume particles. Our derivation breaks down at this point. Thus we focus on the range $m<E^{*}<5 m$, within which it turns out that the only infinite-volume observables that enter are quantities related to two-to-two and three-to-three scattering $2^{2}$

An additional technical requirement is that the two-particle K-matrix remain finite in the kinematical range of interest. This range runs from $0<E_{2}^{*}<4 m$, where $E_{2}^{*}$ is the two-particle CM energy. Thus this region extends well below the threshold at $E_{2}^{*}=2 \mathrm{~m}$, requiring appropriate analytic continuation of the K-matrix. This requirement means that $|\delta|<\pi / 2$ for all angular momenta below the four-particle threshold-the interactions can be neither attractive enough to produce a resonance nor overly repulsive.

We next establish our notation for three-particle kinematics, considering first the case where all particles are on shell. If the momenta of two of these particles are $\vec{k}$ and $\vec{a}$, then that of the third is fixed to be $\vec{b}_{k a} \equiv \vec{P}-\vec{k}-\vec{a}$ by momentum conservation. The corresponding energies are denoted

$$
\omega_{k}=\sqrt{\vec{k}^{2}+m^{2}}, \omega_{a}=\sqrt{\vec{a}^{2}+m^{2}}, \text { and } \omega_{k a}=\sqrt{(\vec{P}-\vec{k}-\vec{a})^{2}+m^{2}} \text {, respectively. }
$$

The momenta $\vec{k}$ and $\vec{a}$ cannot be chosen freely: on-shell and total energy constraints require

$$
E=\omega_{k}+\omega_{a}+\omega_{k a}
$$

It is convenient to separate the three particles into a "spectator", which we take to be that with momentum $\vec{k}$, and the remaining two-particle pair, with four-momentum $P_{2}=$ $\left(E-\omega_{k}, \vec{P}-\vec{k}\right)$. The energy of this pair in its CM-frame (which we stress is different, in general, from the CM-frame of all three particles) is labeled $E_{2, k}^{*}$, where

$$
E_{2, k}^{* 2}=\left(P_{2}\right)^{2}=\left(E-\omega_{k}\right)^{2}-(\vec{P}-\vec{k})^{2}
$$

\footnotetext{
${ }^{2}$ Were we to remove the $\mathbb{Z}_{2}$ symmetry, we would also need to include two-to-three amplitudes, as has been done in Ref. 138.
} 
For Eq. 5.2 to hold, we must have that $E_{2, k}^{*} \geq 2 m$. For fixed total energy-momentum, this condition holds only for a finite regions of $\vec{k}$.

We now boost to the two-particle CM frame, which requires a boost velocity of

$$
\vec{\beta}_{k} \equiv-\frac{\vec{P}-\vec{k}}{E-\omega_{k}}
$$

We denote by $\left(\omega_{a}^{*}, \vec{a}^{*}\right)$ and $\left(\omega_{k a}^{*}, \vec{b}_{k a}^{*}\right)$ the four vectors reached by boosting $\left(\omega_{a}, \vec{a}\right)$ and $\left(\omega_{k a}, \vec{b}_{k a}\right)$, respectively. If Eq. 5.2 holds, then we have

$$
\omega_{a}^{*}=\omega_{k a}^{*}=\frac{E_{2, k}^{*}}{2} \text { and } \vec{a}^{*}=-\vec{b}_{k a}^{*}
$$

while the magnitudes of the momenta in the two-particle CM frame satisfy

$$
a^{*}=b_{k a}^{*}=q_{k}^{*} \equiv \sqrt{E_{2, k}^{* 2} / 4-m^{2}} .
$$

Thus, once $(E, \vec{P})$ and $\vec{k}$ are fixed, the remaining degrees of freedom for three on-shell particles can be labeled by a single unit vector, $\hat{a}^{*}$. This is simply the direction of motion for one of the two non-spectator particles in their two-particle CM frame. We will often parametrize the dependence on this direction in terms of spherical harmonics.

We can also interchange the roles of $\vec{k}$ and $\vec{a}$, treating the latter as the spectator. In this case the CM energy of the non-spectator pair is $E_{2, a}^{*}$ where

$$
E_{2, a}^{* 2} \equiv\left(E-\omega_{a}\right)^{2}-(\vec{P}-\vec{a})^{2}
$$

while the required boost has velocity

$$
\vec{\beta}_{a}=-\frac{\vec{P}-\vec{a}}{E-\omega_{a}} .
$$

This boost leads to $\left(\omega_{k}, \vec{k}\right) \rightarrow\left(\omega_{k}^{*}, \vec{k}^{*}\right)$, and the on-shell condition implies

$$
k^{*}=q_{a}^{*} \equiv \sqrt{E_{2, a}^{* 2} / 4-m^{2}},
$$

so that the three on-shell particles [with fixed $(E, \vec{P})]$ are parametrized by $\vec{a}, \hat{k}^{*}$. This discussion exemplifies the notation that we will use repeatedly below, wherein the subscripts denote which momentum is that of the spectator, and it is clear from the context in which two-particle CM frame starred quantities are defined. 
Also relevant are situations in which two of the particles, say those with momenta $\vec{k}$ and $\vec{a}$, are on shell, while the third is not. The energy-momentum of the third particle is then $\left(E-\omega_{k}-\omega_{a}, \vec{b}_{k a}\right)$. As long as $E_{2, k}^{* 2}>0$, we can still boost to the two-particle CM-frame (with boost velocity $\vec{\beta}_{k}$ ), leading to

$$
\left(\omega_{a}, \vec{a}\right) \longrightarrow\left(\omega_{a}^{*}, \vec{a}^{*}\right) \text {, and }\left(E-\omega_{k}-\omega_{a}, \vec{b}_{k a}\right) \longrightarrow\left(E_{2, k}^{*}-\omega_{a}^{*},-\vec{a}^{*}\right) \text {. }
$$

In this case, however, $a^{*} \neq q_{k}^{*}$, so the degrees of freedom are now parametrized by $\vec{k}$ and the vector $\vec{a}^{*}$. As in the on-shell case, also here we can exchange the roles of $\vec{k}$ and $\vec{a}$. As long as $E_{2, a}^{* 2}>0$, we can boost $\left(\omega_{k}, \vec{k}\right)$ by $\vec{\beta}_{a}$ to define $\left(\omega_{k}^{*}, \vec{k}^{*}\right)$, with $k^{*}$ now unconstrained.

We use these coordinates to express the momentum dependence of the on-shell quantities appearing in the final result. We start with two-to-two scattering, which occurs as a subprocess within the larger three-to-three interactions. We denote the two-to-two scattering amplitude by $\mathcal{M}_{2}$ and the corresponding K-matrix ${ }^{3}$ by $\mathcal{K}_{2}$. Assuming the particle with momentum $\vec{k}$ is the unscattered spectator, an appropriate functional dependence is $\mathcal{M}_{2}\left(\vec{k}, \hat{a}^{\prime *}, \hat{a}^{*}\right)$ and $\mathcal{K}_{2}\left(\vec{k}, \hat{a}^{\prime *}, \hat{a}^{*}\right)$. In each, the role of the first argument is kinematic. Knowing the spectator momentum, as well as the total energy-momentum, one can determine the lab-frame total momentum of the scattering pair $\left[\left(E-\omega_{k}, \vec{P}-\vec{k}\right)\right]$ as well as the boost velocity $\vec{\beta}_{k}$ needed to move to the scattering CM-frame. In the latter frame, $\hat{a}^{*}$ and $\hat{a}^{\prime *}$ are, respectively, the initial and final directions of one of the scattered particles. Decomposing the dependence on these directions into spherical harmonics, we write

$$
\mathcal{K}_{2}\left(\vec{k}, \hat{a}^{\prime *}, \hat{a}^{*}\right)=4 \pi Y_{\ell^{\prime}, m^{\prime}}^{*}\left(\hat{a}^{\prime *}\right) \mathcal{K}_{2 ; \ell^{\prime}, m^{\prime} ; \ell, m}(\vec{k}) Y_{\ell, m}\left(\hat{a}^{*}\right)
$$

and similarly for $\mathcal{M}_{2}$. Here and in the following there is an implicit sum over repeated indices. The factor of $4 \pi$ is conventional [130]. Rotational invariance implies that $\mathcal{K}_{2 ; \ell^{\prime}, m^{\prime} ; \ell, m}(\vec{k}) \propto$ $\delta_{\ell^{\prime}, \ell} \delta_{m^{\prime}, m}$, and that for each $\ell$ there is only one independent physical quantity, the scattering phase-shift in the given partial wave.

\footnotetext{
${ }^{3}$ Our K-matrix $\mathcal{K}_{2}$ is standard above threshold, while below threshold it is defined by analytic continuation. This is discussed further below [see Eqs. [5.32, [5.101) and [5.102]].

${ }^{4}$ This is the only exception to our notation involving superscript $*$. The $*$ on $Y_{\ell m}^{*}$ indicates complex conjugation.
} 
Now we turn to three-to-three scattering. Although our final quantization condition contains a three-particle K-matrix, we first discuss the standard three-to-three scattering amplitude, $\mathcal{M}_{3}$. This allows us to describe a new issue that arises with three particles in a more familiar context. As usual, $\mathcal{M}_{3}$ is the sum of all connected six-point diagrams with external legs amputated and on shell. We write its functional dependence as $\mathcal{M}_{3}\left(\vec{k}^{\prime}, \hat{a}^{\prime *}, \vec{k}, \hat{a}^{*}\right)$, where now the "spectator" momentum changes from the initial $(\vec{k})$ to the final $\left(\vec{k}^{\prime}\right)$ state. The two direction vectors $\hat{a}^{*}$ and $\hat{a}^{\prime *}$ are defined in the corresponding two-particle CM frames, which are different for the initial and final states. We stress that $\mathcal{M}_{3}$ is symmetric under particle interchange separately in the initial and final states, so that the choice of spectator is arbitrary. We use asymmetric coordinates because of the presence of two-to-two scatterings.

We would like to decompose $\mathcal{M}_{3}$ into spherical harmonics, as in Eq. (5.11). Although we can do this formally, we do not expect the sum over angular momenta to converge uniformly. This is because of a complication not present in the two-to-two case: the threeto-three scattering amplitude has physical singularities above threshold 5 These singularities have nothing to do with bound states, but are instead due to the possibility of two particles scattering and then traveling arbitrarily far before one of them scatters off the third particle (see Fig. 5.1). The three-particle interaction can thus become arbitrarily non-local. This means that, even at low energies, a truncation of the angular momentum sum is not justified, since a truncated expansion will give a function that is everywhere finite. Because truncation is crucial for practical applications of the quantization condition, we must find a way around this problem.

Our solution is to introduce an intermediate quantity that has the same singularities as the three-to-three scattering amplitude but depends only on the on-shell two-to-two amplitude $\mathcal{M}_{2}$. This is possible because divergences in the three-to-three scattering amplitude are always due to diagrams with only pairwise scatterings, with all intermediate states on shell ${ }^{6}$

\footnotetext{
${ }^{5}$ The properties and physical consequences of these singularities are discussed, for example, in Refs. [240, 241, 242, 243.

${ }^{6}$ Indeed, a diagram with $n$ two-to-two scatterings is divergent if and only if it is kinematically possible to have $n$ classical pairwise scatterings (not counting events with zero momentum transfer). For degenerate
} 


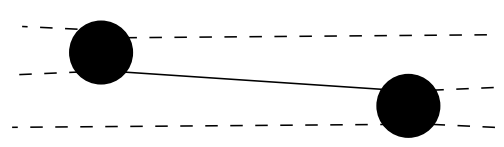

Figure 5.1: Example of singular contribution to the on-shell three-to-three scattering amplitude. Dashed lines are on-shell, amputated, external propagators, while the solid line is a fully dressed propagator, which can in general be off-shell. Filled circles represent twoto-two scattering amplitudes. The internal (solid) line can become on shell for physical external momenta, corresponding to two isolated two-to-two scattering events.

Labeling this intermediate quantity $\mathcal{M}_{\text {sing,3 }}$, we define the "divergence-free" amplitude by

$$
\mathcal{M}_{\mathrm{df}, 3}\left(\vec{k}^{\prime}, \hat{a}^{\prime *}, \vec{k}, \hat{a}^{*}\right) \equiv \mathcal{M}_{3}\left(\vec{k}^{\prime}, \hat{a}^{\prime *}, \vec{k}, \hat{a}^{*}\right)-\mathcal{M}_{\mathrm{sing}, 3}\left(\vec{k}^{\prime}, \hat{a}^{\prime *}, \vec{k}, \hat{a}^{*}\right)
$$

This is shown diagrammatically in Fig. 5.2. By construction, $\mathcal{M}_{\mathrm{df}, 3}$ is a smooth function, and therefore has a uniformly convergent partial-wave expansion:

$$
\mathcal{M}_{\mathrm{df}, 3}\left(\vec{k}^{\prime}, \hat{a}^{\prime *}, \vec{k}, \hat{a}^{*}\right)=4 \pi Y_{\ell^{\prime}, m^{\prime}}^{*}\left(\hat{a}^{\prime *}\right) \mathcal{M}_{\mathrm{df}, 3 ; \ell^{\prime}, m^{\prime} ; \ell, m}\left(\vec{k}^{\prime}, \vec{k}\right) Y_{\ell, m}\left(\hat{a}^{*}\right)
$$

The singular part, $\mathcal{M}_{\text {sing, } 3}$, must be included without partial-wave decomposition. A diagrammatic definition of $\mathcal{M}_{\text {sing,3 }}$ in sketched in Fig. 5.2, it can be defined formally as the solution to an integral equation. Since we do not need this quantity in this work we do not go into the details here.

As already noted above, our quantization condition depends not on $\mathcal{M}_{\mathrm{df}, 3}$ but rather on a closely related K-matrix-like quantity $\mathcal{K}_{\mathrm{df}, 3}$. Roughly speaking, this is built up of the same Feynman diagrams as $\mathcal{M}_{\mathrm{df}, 3}$, and has the above-threshold divergence removed in a similar way. However, to define $\mathcal{K}_{\mathrm{df}, 3}$ a modified principal-value (PV) pole prescription is used instead of the $i \epsilon$ prescription, and there are some additional subtleties. Thus we delay

particles only three scatterings are possible so there are two divergent diagrams. For non-degenerate particles further scatterings are possible. This is explained in Ref. [240]. As we will find, our derivation requires that we subtract all the diagrams that are needed to render the non-degenerate $\mathcal{M}_{\mathrm{df}, 3}$ finite, even though all but two of these are finite for the degenerate case we study. 


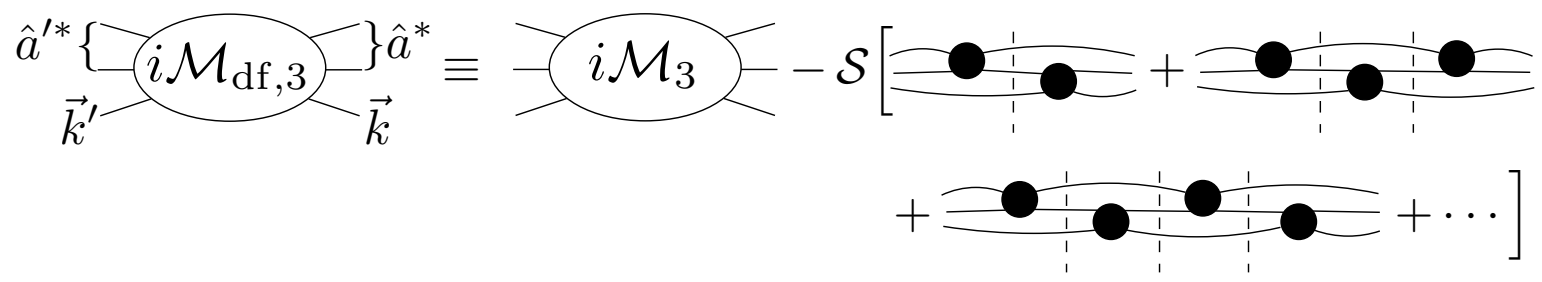

Figure 5.2: Diagrammatic definition of the divergence-free three-to-three amplitude, $\mathcal{M}_{\mathrm{df}, 3}$. In the subtracted term, filled circles represent on-shell two-to-two scattering amplitudes $\mathcal{M}_{2}$. Dashed cuts stand for simple kinematic factors that appear between adjacent $\mathcal{M}_{2}$. These factors have the requisite poles so that the subtracted terms cancel the singularities in $\mathcal{M}_{3}$. The $\mathcal{S}$ outside the square brackets indicates that the subtracted terms are symmetrized.

a full definition until we present the derivation of the quantization condition. What matters here is that $\mathcal{K}_{\mathrm{df}, 3}$ is a non-singular, infinite-volume quantity, closely related to the scattering amplitude. It is also separately symmetric under initial and final particle interchange. Its functional dependence and harmonic decomposition is as for $\mathcal{M}_{\mathrm{df}, 3}$ :

$$
\mathcal{K}_{\mathrm{df}, 3}\left(\vec{k}^{\prime}, \hat{a}^{\prime *}, \vec{k}, \hat{a}^{*}\right)=4 \pi Y_{\ell^{\prime}, m^{\prime}}^{*}\left(\hat{a}^{\prime *}\right) \mathcal{K}_{\mathrm{df}, 3 ; \ell^{\prime}, m^{\prime} ; \ell, m}\left(\vec{k}^{\prime}, \vec{k}\right) Y_{\ell, m}\left(\hat{a}^{*}\right)
$$

We stress that $\mathcal{K}_{\mathrm{df}, 3 ; \ell^{\prime}, m^{\prime} ; \ell, m}\left(\vec{k}^{\prime}, \vec{k}\right)$ is not diagonal in $\ell$ or $m$, since two-particle angular momentum is not a good quantum number in three-to-three scattering. It is also noteworthy that our derivation of the quantization condition automatically leads to removal of the divergent part from $\mathcal{K}_{\mathrm{df}, 3}$. Thus not only is the subtraction reasonable from the perspective of defining useful infinite-volume observables (i.e. allowing a convergent partial-wave expansion) it also arises naturally in our investigation of the finite-volume theory.

We are now in a position to present the quantization condition: a relation between $\mathcal{K}_{2}, \mathcal{K}_{\mathrm{df}, 3}$ and the finite-volume spectrum. This relation involves three-particle phase space restricted by the constraint of finite-volume. In particular, we need $\mathcal{K}_{2 ; \ell^{\prime}, m^{\prime} ; \ell, m}(\vec{k})$ and $\mathcal{K}_{\mathrm{df}, 3 ; \ell^{\prime}, m^{\prime} ; \ell, m}\left(\vec{k}^{\prime}, k\right)$ only for $\vec{k}, \vec{k}^{\prime} \in(2 \pi / L) \mathbb{Z}^{3}$. We therefore define the finite-volume restric- 
tions of these amplitudes

$$
\begin{aligned}
\mathcal{K}_{2 ; k^{\prime}, \ell^{\prime}, m^{\prime} ; k, \ell, m} & \equiv \delta_{k^{\prime}, k} \mathcal{K}_{2 ; \ell^{\prime}, m^{\prime} ; \ell, m}(\vec{k}) \quad \text { for } \quad \vec{k} \in(2 \pi / L) \mathbb{Z}^{3}, \\
\mathcal{K}_{\mathrm{df}, 3 ; k^{\prime}, \ell^{\prime}, m^{\prime} ; k, \ell, m} & \equiv \mathcal{K}_{\mathrm{df}, 3 ; \ell^{\prime}, m^{\prime} ; \ell, m}\left(\vec{k}^{\prime}, \vec{k}\right) \quad \text { for } \quad \vec{k}^{\prime}, \vec{k} \in(2 \pi / L) \mathbb{Z}^{3} .
\end{aligned}
$$

The left-hand sides of these equations are to be viewed as matrices in an extended space with indices 7

[finite volume momentum $\left.\vec{k} \in(2 \pi / L) \mathbb{Z}^{3}\right] \times$ [two particle angular momentum]

All other quantities entering our final result will also be matrices acting on this space.

The finite-volume spectrum is determined by

$$
\operatorname{det}\left[1+F_{3} \mathcal{K}_{\mathrm{df}, 3}\right]=0
$$

where the determinant is over the direct product space just introduced. The matrix $F_{3}$ is

$$
F_{3} \equiv \frac{F}{2 \omega L^{3}}\left[-\frac{2}{3}+\frac{1}{1+\left[1+\mathcal{K}_{2} G\right]^{-1} \mathcal{K}_{2} F}\right]
$$

where

$$
\begin{aligned}
{\left[\frac{1}{2 \omega L^{3}}\right]_{k^{\prime}, \ell^{\prime}, m^{\prime} ; k, \ell, m} } & \equiv \delta_{k^{\prime}, k} \delta_{\ell^{\prime}, \ell} \delta_{m^{\prime}, m} \frac{1}{2 \omega_{k} L^{3}}, \\
G_{p, \ell^{\prime}, m^{\prime} ; k, \ell, m} & \equiv\left(\frac{k^{*}}{q_{p}^{*}}\right)^{\ell^{\prime}} \frac{4 \pi Y_{\ell^{\prime}, m^{\prime}}\left(\hat{k}^{*}\right) H(\vec{p}) H(\vec{k}) Y_{\ell, m}^{*}\left(\hat{p}^{*}\right)}{2 \omega_{k p}\left(E-\omega_{k}-\omega_{p}-\omega_{k p}\right)}\left(\frac{p^{*}}{q_{k}^{*}}\right)^{\ell} \frac{1}{2 \omega_{k} L^{3}}, \\
F_{k^{\prime}, \ell^{\prime}, m^{\prime} ; k, \ell, m} & \equiv \delta_{k^{\prime}, k} F_{\ell^{\prime}, m^{\prime} ; \ell, m}(\vec{k}) \\
F_{\ell^{\prime}, m^{\prime} ; \ell, m}(\vec{k}) & =F_{\ell^{\prime}, m^{\prime} ; \ell, m}^{i \epsilon}(\vec{k})+\rho_{\ell^{\prime}, m^{\prime} ; \ell, m}(\vec{k}), \\
F_{\ell^{\prime}, m^{\prime} ; \ell, m}^{i \epsilon}(\vec{k}) & =\frac{1}{2}\left[\frac{1}{L^{3}} \sum_{\vec{a}}-\int_{\vec{a}}\right] \frac{4 \pi Y_{\ell^{\prime}, m^{\prime}}\left(\hat{a}^{*}\right) Y_{\ell, m}^{*}\left(\hat{a}^{*}\right) H(\vec{k}) H(\vec{a}) H\left(\vec{b}_{k a}\right)}{2 \omega_{a} 2 \omega_{k a}\left(E-\omega_{k}-\omega_{a}-\omega_{k a}+i \epsilon\right)}\left(\frac{a^{*}}{q_{k}^{*}}\right)^{\ell+\ell^{\prime}}
\end{aligned}
$$

${ }^{7}$ Our notation for the momentum indices, $k$ and $k^{\prime}$, is somewhat imprecise. These each are stand-ins for three-dimensional integer vectors labeling the allowed finite-volume momenta. In other words, whenever a spectator momentum occurs as an index, it indicates implicitly that the corresponding three-vector momentum is one of those allowed in finite volume. 
where $\int_{\vec{a}} \equiv \int d^{3} a /(2 \pi)^{3}$ and the sum over $\vec{a}$ in $F$ runs over all finite-volume momenta. Here $\rho$ is a phase-space factor defined by

$$
\begin{aligned}
& \rho_{\ell^{\prime}, m^{\prime} ; \ell, m}(\vec{k}) \equiv \delta_{\ell^{\prime}, \ell} \delta_{m^{\prime}, m} H(\vec{k}) \widetilde{\rho}\left(P_{2}\right), \\
& \widetilde{\rho}\left(P_{2}\right) \equiv \frac{1}{16 \pi \sqrt{P_{2}^{2}}} \times \begin{cases}-i \sqrt{P_{2}^{2} / 4-m^{2}} & (2 m)^{2}<E_{2, k}^{* 2}, \\
\left|\sqrt{P_{2}^{2} / 4-m^{2}}\right| & 0<E_{2, k}^{* 2} \leq(2 m)^{2},\end{cases}
\end{aligned}
$$

where we recall that $P_{2}$ is the four-momentum of the non-spectator pair. Finally, $H$ is a smooth cut-off function to be defined shortly.

The quantization condition Eq. (5.18) is our main result, and will be derived in Sec. 5.3. Here we work our way through the definitions, explaining the origin and meaning of each contribution. As noted above, $\mathcal{K}_{\mathrm{df}, 3}$ is closely related to the divergence-free part of the full three-to-three scattering amplitude. The singular parts of this amplitude end up in the quantity $F_{3}$, where they lead to chains of the form $\ldots \mathcal{K}_{2} G \mathcal{K}_{2} G \mathcal{K}_{2} \ldots$ which are obtained by expanding out $\left[1+\mathcal{K}_{2} G\right]^{-1} \mathcal{K}_{2}$. These chains arise from subtraction terms like those in Fig. 5.2, with the filled circles now representing on-shell K-matrices $\mathcal{K}_{2}$ (rather than $\mathcal{M}_{2}$ ). The singular "cuts" between K-matrices give rise to the kinematical factors $G$.

In the definition of $G$, Eq. (5.21), we are using the notation described in Eqs. (5.1)-(5.10), with $\vec{p}$ in place of $\vec{a}$. Observe in particular that $G$ makes use of the off-shell phase-space described in the paragraph containing Eq. 5.10 . Since both $\vec{k}$ and $\vec{p}$ can equal any finitevolume three momentum, $\left(E-\omega_{k}-\omega_{p}, \vec{b}_{p k}\right)$ will generally not be on shell. For this reason the magnitude of $\vec{k}^{*}$ (defined via a boost with velocity $\vec{\beta}_{p}$ ) and that of $\vec{p}^{*}$ (boost velocity $\vec{\beta}_{k}$ ) are unconstrained. These magnitudes appear in the factors $\left(k^{*} / q_{p}^{*}\right)^{\ell^{\prime}}$ and $\left(p^{*} / q_{k}^{*}\right)^{\ell}$, which remove singularities due to the spherical harmonics and so ensure that $G$ is non-singular for $\vec{k}^{*}$ or $\vec{p}^{*}$ equal to zero. (A similar factor $\left(a^{*} / q_{k}^{*}\right)^{\ell+\ell^{\prime}}$ appears in $F$ for the same reason.)

The final ingredient in $G$ is the function $H$ (which appears also in $F$ ). The role of $H$ is to provide a smooth ultraviolet cut-off on the sum over spectator momentum. There are two cut-off functions, $H(\vec{p})$ and $H(\vec{k})$, because $G$ has different spectator momenta in its leftand right-handed indices ( $\vec{p}$ and $\vec{k}$, respectively). To understand the need for the cut-off we note that, for fixed $(E, \vec{P})$, as the spectator momentum (say $\vec{k}$ ) increases in magnitude, the energy-momentum of the other two particles falls below threshold, $E_{2, k}^{*}<2 m$. Now, in the 
quantization condition (5.18), the determinant runs over all values of spectator momentum, which leads to values of $E_{2, k}^{* 2}$ arbitrarily far below threshold. Once $E_{2, k}^{* 2} \leq 0$, however, the boost needed to define $p^{*}$ becomes unphysical $\left(\left|\beta_{k}\right| \geq 1\right)$. The cut-off function $H(\vec{k})$ resolves this issue. It has the properties

$$
H(\vec{k})= \begin{cases}0, & E_{2, k}^{* 2} \leq 0 \\ 1, & (2 m)^{2}<E_{2, k}^{* 2}\end{cases}
$$

where the first condition removes unphysical boosts and the second ensures that the cut-off does not change the contributions from on-shell intermediate states. In the intermediate region, $0<E_{2, k}^{* 2}<(2 m)^{2}, H(\vec{k})$ interpolates between 0 and 1 . For reasons that will become clear in the derivation below, this interpolation must be smooth. An example of a function which does the job is

$$
H(\vec{k}) \equiv J\left(E_{2, k}^{* 2} /\left[4 m^{2}\right]\right)
$$

with

$$
J(x) \equiv \begin{cases}0, & x \leq 0 \\ \exp \left(-\frac{1}{x} \exp \left[-\frac{1}{1-x}\right]\right), & 0<x \leq 1 ; \\ 1, & 1<x .\end{cases}
$$

This function is plotted in Fig. 5.3 .

It would also be consistent with the requirements stated so far to have $H$ remain smooth but transition more rapidly from 0 to 1 . In that case, however, the difference between a sum and an integral over $H$ will be enhanced

$$
\left[\frac{1}{L^{3}} \sum_{\vec{k}}-\int_{\vec{k}}\right] H(\vec{k})=\mathcal{O}\left(e^{-\Delta L}\right),
$$

with $\Delta$ the width of the drop-off region. Since these corrections are neglected, an enhancement from using too small a width would invalidate our final result. We must thus additionally require

$$
\left[\frac{1}{L^{3}} \sum_{\vec{k}}-\int_{\vec{k}}\right] H(\vec{k})=\mathcal{O}\left(e^{-m L}\right) .
$$

In other words we must ensure that $m$ is the smallest energy scale in the problem, and thus take $\Delta \approx m$. The form sketched in Fig. 5.3 satisfies this requirement. 


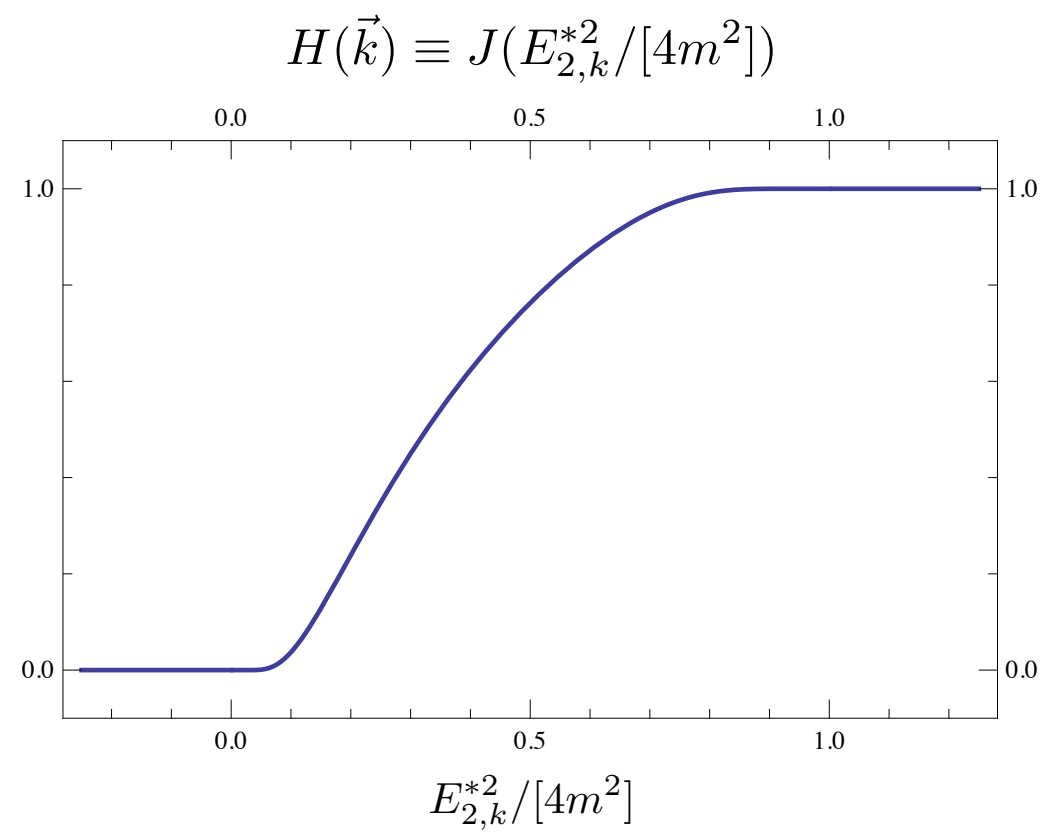

Figure 5.3: Plot of the smooth cutoff function $H(\vec{k}) \equiv J\left(E_{2, k}^{* 2} /\left[4 m^{2}\right]\right)$. The function varies from 0 to 1 as $E_{2, k}^{* 2} \equiv\left(E-\omega_{k}\right)^{2}-(\vec{P}-\vec{k})^{2}$ varies from 0 to $4 m^{2}$. Using this range ensures that the function has width $\Delta \approx m$ in the space of spectator momentum $\vec{k}$. 
The appearance of sub-threshold momenta is a general feature of the three-particle quantization condition, as first pointed out in Ref. [138]. Indeed, for spectator momenta such that $0<E_{2, k}^{* 2}<(2 m)^{2}$, the two-particle K-matrices in $F_{3}$ are evaluated below threshold. Our modified PV prescription [denoted $\widetilde{\mathrm{PV}}$ and defined in Eqs. 5.59 and 5.66 below] ensures that this is achieved by analytic continuation. 8 The cut-off functions in $G$ (and in $F$ ) ensure that these sub-threshold contributions are absent for $E_{2, k} \leq 0$. The three-particle amplitude $\mathcal{K}_{\mathrm{df}, 3}$ must also be evaluated for sub-threshold momenta, which is achieved by analytic continuation.

The final matrix that enters the quantization condition is $F$. This is the kinematic factor that brings in finite-volume effects. Its definition uses the notation introduced in Eqs. (5.1)-(5.10). As shown in Eq. (5.22), it is diagonal in spectator momentum, and is thus a essentially a two-particle quantity. Indeed, the matrix $F^{i \epsilon}(\vec{k})$ defined in Eq. 5.24) is essentially the same as the kinematic quantity of the same name introduced in Ref. [130] in the formulation of the two-particle quantization condition in a moving frame. The precise relation, given in Eq. D.10 in Appendix D, allows $F^{i \epsilon}$ to be written in terms of generalizations of the zeta-functions introduced in Refs. [127, 128]. The only difference between our $F^{i \epsilon}$ and that of Ref. [130] is that we use a different ultraviolet cut-off-our cut-off is provided by the product of three $H$ functions. This change in cut-off leads, however, to differences which are exponentially suppressed as $L \rightarrow \infty$.

The kinematic factor which enters the quantization condition is $F$ rather than $F^{i \epsilon}$. The difference between these two quantities, given by Eq. (5.23), is the phase space factor $\rho$, a quantity that appears repeatedly in the derivation of Sec. 5.3 and which is diagonal in angular momentum. For example, the relation between the two-particle scattering amplitude and K-matrix is [see Eq. (5.102)]

$$
\mathcal{M}_{2}^{-1}=\mathcal{K}_{2}^{-1}+\rho
$$

\footnotetext{
${ }^{8}$ This is in distinction to the standard PV prescription, which leads to a cusp in $\mathcal{K}_{2}$ at threshold. Our definition is the same as that used in studies of bound-state energies using Lüscher's two-particle quantization condition (see, e.g., Refs. [244, 143]). In particular, the quantity $\left(a^{*}\right)^{2 \ell+1} \cot \delta_{\ell}\left(a^{*}\right)$ has a Taylor expansion in $\left(a^{*}\right)^{2}$ that can be analytically continued to $\left(a^{*}\right)^{2}<0$.
} 
The $\rho$ term in $F$ arises because of our use of a modified PV pole prescription. As can be seen from Eq. 5.24, $F^{i \epsilon}$ is the difference between a sum and integral of three-particle cut, with the integral defined using the $i \epsilon$ prescription. The $\rho$ term in Eq. 5.23 is exactly what is needed so that $F$ itself is the sum-integral difference with the integral defined by the $\widetilde{\mathrm{PV}}$ prescription. The latter is the quantity that appears naturally in our derivation. This means that $F$ is real. In addition, as we move below threshold $\left[E_{2, k}^{* 2}<(2 m)^{2}\right]$, while $F^{i \epsilon}$ drops to zero rapidly, since the summand/integrand is no longer singular, $\rho$ (and thus $F$ ) grows since $\left|q_{k}^{*}\right|$ is increasing. Eventually, however, as $E_{2, k}^{* 2}$ approaches zero, this growth is overcome by the decrease in the cut-off function $H$, such that $\rho$ vanishes for $E_{2, k}^{* 2} \leq 0$.

The quantization condition (5.18) is similar in form to that for two particles [see Eq. (5.100) below, as well as Refs. [129, 130, 140, 146]]. In principle, they are both to be used in the same way: if one knows the scattering amplitudes $\mathcal{K}_{2}$ and $\mathcal{K}_{\mathrm{df}, 3}$ then, for a given choice of $\left\{L, n_{\vec{P}}\right\}$, the quantization conditions predict the finite-volume energy levels. Of course, what we are really interested in is inverting this prediction, i.e. using numerically determined energy levels to extract information about infinite-volume scattering amplitudes. This more challenging task is discussed in the following section.

\subsection{Truncating the quantization condition}

In this section we discuss how one might use the three-particle quantization condition, Eq. (5.18), in practice. Specifically, we assume that, using lattice simulations, one has determined some number of three-particle energy levels for a set of choices of $\{L, \vec{P}\}$. From this information, we want to learn as much as possible about $\mathcal{K}_{\mathrm{df}, 3}$.

The first step is to assume that, using Lüscher's two-particle quantization condition and

its generalizations, the two-particle K-matrix $\mathcal{K}_{2 ; \ell^{\prime}, m^{\prime} ; \ell, m}(\vec{k})$ has been determined. To do so in practice one must assume that $\mathcal{K}_{2}$ is negligible for large enough angular momenta, which is a good approximation for a given two-particle energy. Specifically, we assume $\mathcal{K}_{2}=0$ for $\ell>\ell_{\max , 2}$. In this case the two-particle quantization condition is truncated to a solvable finite matrix condition. In addition, since lattice results inevitably determine the $\mathcal{K}_{2}$ (or, equivalently, the phase shifts) only for a discrete set of kinematical points, we assume that 
these have been suitably interpolated and/or extrapolated to obtain continuous functions.

In the three-particle case, we are dealing with a larger index space, containing the additional sum over finite-volume spectator momenta. However, the regulator function $H$ provides an automatic truncation of this sum. This occurs because, for fixed $(E, \vec{P})$, there are a finite number of values of $\vec{k}$ for which $H(\vec{k})$ is non-vanishing. We call this number of values $N$. This automatically truncates $G$ and $F$ (which contain $H(\vec{k})$ ) to be $N \times N$ matrices in spectator-momentum space, with all other entries vanishing. Since $\mathcal{K}_{2}$ always sits between factors of $F$ and $G$ [as can be seen by expanding out the nested geometric series in Eq. [5.19] ], $\mathcal{K}_{2}$ is also effectively truncated (in the sense that the terms in $\mathcal{K}_{2}$ lying outside the $N \times N$ block do not contribute). Since $F_{3}$ always has an $F$ at both ends (again after expanding out), it also is truncated. Finally, expanding out the determinant (e.g. using $\operatorname{det} Z=\exp \operatorname{Tr} \ln Z$ ) one sees that $\mathcal{K}_{\mathrm{df}, 3}$ always has an $F_{3}$ on both sides and so it also is effectively truncated $?^{9}$

Next we consider the spherical harmonic indices. As already noted, we assume $\mathcal{K}_{2}$ is truncated in these indices at $\ell_{\max , 2}$. To reduce the determinant condition to a finitedimensional space, we must further assume that $\mathcal{K}_{\mathrm{df}, 3}$ is truncated, in both $\ell$ and $\ell^{\prime}$, at $\ell_{\max , 3}$. This is reasonable because $\mathcal{K}_{\mathrm{df}, 3}$ is a smooth function, as is made clear in the coarse of defining it, in Sec. 5.3 below. Defining $\ell_{\max }$ as the larger of $\ell_{\max , 2}$ and $\ell_{\max , 3}$, we find that all factors of $F$ and $G$ appearing in the quantization-condition are projected onto a $\left(2 \ell_{\max }+1\right) \times\left(2 \ell_{\max }+1\right)$ subspace of the angular-momentum space. This follows from the argument already given above: expanding in $F$ and $G$, one finds that every factor of these two kinematic matrices sits between (and is thus truncated by) factors of either $\mathcal{K}_{2}$ or $\mathcal{K}_{\mathrm{df}, 3}$. The net result is that the quantization condition collapses to that for truncated matrices of size $\left(2 \ell_{\max }+1\right) N \times\left(2 \ell_{\max }+1\right) N$. In this way the formal result has been turned into something more practical 10

\footnotetext{
${ }^{9}$ The fact that the sum over $\vec{k}$ is truncated makes sense in the limit of weak interactions. If all interactions vanish, then, for given $\vec{P}$, there will only be a finite number of free three-particle states with energies below, or in the vicinity of, any given choice for $E$. It is primarily these states which are mixed by interactions to form the finite-volume eigenstates with $E_{i}<E$.

${ }^{10}$ We suspect that it is inconsistent to choose $\ell_{\max , 3}<\ell_{\max , 2}$, because three-particle scattering involves
} 
The final step is to assume a parametrization of the $\vec{k}^{\prime}$ and $\vec{k}$ dependence of the non-zero angular-momentum components of $\mathcal{K}_{\mathrm{df}, 3}$. We stress again that $\mathcal{K}_{\mathrm{df}, 3}$ is not diagonal in its angular-momentum indices (unlike $\mathcal{K}_{2}$ ) so that there will be quite a number of components to parametrize. Nevertheless, given a finite number of parameters, and knowledge of $\mathcal{K}_{2}$ (including analytic continuation below threshold), each of the measured energy levels gives, when inserted into the quantization condition, a relation between the parameters. Nonetheless, given knowledge of $\mathcal{K}_{2}$ (including analytic continuation below threshold), each of the measured three-particle energy levels gives a relation between the parameters characterizing $\mathcal{K}_{\mathrm{df}, 3}$. Thus, given enough energy levels one can solve for any finite set of parameters. Although this sounds complicated, we note that the recent kaon resonance study of Ref. [92] was able to deal with multiple (two-particle) channels using a suitable parametrization and many energy levels.

We close this section by working out the simplest possible case of the above-described program. We assume that both $\mathcal{K}_{2}$ and $\mathcal{K}_{\mathrm{df}, 3}$ are s-wave dominated (i.e. $\ell_{\max , 2}=\ell_{\max , 3}=0$ ), and that $\mathcal{K}_{\mathrm{df}, 3}$ is a function only of the total three-particle $\mathrm{CM}$ energy. These assumptions are summarized by

$$
\mathcal{K}_{2}\left(\vec{k}, \hat{a}^{\prime *}, \hat{a}^{*}\right)=\mathcal{K}_{2}^{s}\left(E_{2, k}^{*}\right) \text { and } \mathcal{K}_{\mathrm{df}, 3}\left(\vec{k}^{\prime}, \hat{a}^{\prime *}, \vec{k}, \hat{a}^{*}\right)=\mathcal{K}_{\mathrm{df}, 3}^{\text {iso }}\left(E^{*}\right)
$$

All matrices entering the quantization condition thus collapse to $N \times N$ matrices in spectatormomentum space, and have the explicit forms

$$
\begin{aligned}
\mathcal{K}_{2 ; k^{\prime}, k}^{s} & \equiv \delta_{k^{\prime}, k} \mathcal{K}_{2}^{s}\left(E_{2, k}^{*}\right), \\
\mathcal{K}_{\mathrm{df}, 3 ; k^{\prime}, k}^{s} & \equiv \mathcal{K}_{\mathrm{df}, 3}^{\mathrm{iso}}\left(E^{*}\right), \\
G_{p, k}^{s} & \equiv \frac{H(\vec{p}) H(\vec{k})}{2 \omega_{k p}\left(E-\omega_{k}-\omega_{p}-\omega_{k p}\right)} \frac{1}{2 \omega_{k} L^{3}}, \\
F_{k^{\prime}, k}^{s} & \equiv \delta_{k^{\prime}, k} \frac{1}{2}\left[\frac{1}{L^{3}} \sum_{\vec{a}}-\int_{\vec{a}}\right] \frac{H(\vec{k}) H(\vec{a}) H\left(\vec{b}_{k a}\right)}{2 \omega_{a} 2 \omega_{k a}\left(E-\omega_{k}-\omega_{a}-\omega_{k a}+i \epsilon\right)}+\delta_{k^{\prime}, k} H(\vec{k}) \widetilde{\rho}\left(P_{2}\right) .
\end{aligned}
$$

two-to-two subprocesses. Indeed the latter are the leading cause of complications in the derivation presented below. The most natural choice appears to us to be $\ell_{\max , 3}=\ell_{\max , 2}$, although we do not know how to demonstrate that this is a rigorous requirement. 
Since $E^{*}$ is fixed, all $N^{2}$ entries of the matrix $\mathcal{K}_{\mathrm{df}, 3 ; k^{\prime}, k}^{s}$ have the same value. It therefore has only one non-zero eigenvalue, $N \mathcal{K}_{\mathrm{df}, 3}^{\text {iso }}\left(E^{*}\right)$. If we work in the basis in which $\mathcal{K}_{\mathrm{df}, 3}^{s}$ is diagonal, then, irrespective of the form of $F_{3}$, the quantization condition 5.18 reduces to the single equation:

$$
1+F_{3}^{\text {iso }} \mathcal{K}_{\mathrm{df}, 3}^{\text {iso }}\left(E^{*}\right)=0
$$

Here

$$
F_{3}^{\text {iso }} \equiv \sum_{\vec{k}, \vec{p}} \frac{1}{2 \omega_{k} L^{3}}\left[F^{s}\left(-\frac{2}{3}+\frac{1}{1+\left[1+\mathcal{K}_{2}^{s} G^{s}\right]^{-1} \mathcal{K}_{2}^{s} F^{s}}\right)\right]_{k, p}
$$

is (up to a factor of $1 / N$ ) the projection of $F_{3}^{s}$ into the subspace spanned by the eigenvector of $\mathcal{K}_{\mathrm{df}, 3}^{s}$ with non-zero eigenvalue. We stress that the sums over $\vec{k}$ and $\vec{p}$ are both truncated to $N$ contributions by the factors of $H$ contained in $F^{s} 11$

The result (5.38) is strikingly simple. If we know $\mathcal{K}_{2}^{s}$ for two-particle CM energies in the range $0<E_{2, k}^{*}<E^{*}-m$, then we can evaluate $F_{3, s}$, a real function depending only on $E$ and $L$. Evaluating this function at a value of $L_{i}$ for which $E_{i}$ is known to be in the finite-volume spectrum, then gives, using Eq. $5.38, \mathcal{K}_{\mathrm{df}, 3}^{\text {iso }}\left(E_{i}^{*}\right)=-1 / F_{3}^{\text {iso }}\left(E_{i}, L_{i}\right)$. This is conceptually very similar to the application of the two-particle quantization condition, which, in the single-channel limit, can be written as $1+F \mathcal{K}_{2}=0$ [see Eq. (5.100) in the following section]. The difference is that the quantity $F_{3}$ contains information about twoparticle scattering, while $F$ is simply a kinematic function. This difference reflects the fact that, in the three-particle case, particles can interact pairwise as well as all together.

One concern one might have about the isotropic approximation and the result (5.38) is that one apparently only obtains a single energy level whereas $N$ free three-particle levels enter the analysis. It thus seems that some finite-volume states have been lost. In fact, all but one of the free states are present once one takes into account that the equality of all $N^{2}$

\footnotetext{
${ }^{11}$ The truncations that enter through the $H$ functions can also be relaxed in the isotropic limit if desired. Recall that $H(\vec{k})$ was required to vanish for $E_{2, k}^{* 2}<0$, see Eq. 5.29 . This is necessary because otherwise the various starred quantities that enter $F$ and $G$ become ill-defined. However, as is clear from Eqs. 5.37) and (5.36), all of these starred quantities are absent in the isotropic limit. Thus $H(\vec{k})$ may have support for $E_{2, k}^{* 2}<0$, as long as $\mathcal{K}_{2}^{s}\left(E_{2, k}^{*}\right)$ is a well-defined smooth function which is known over the energy range included. This extension of $H$ is required to show that our quantization condition reproduces the threshold expansion of Refs. [245, 246]. This check is presented in a separate note, to appear.
} 
elements of the truncated $\mathcal{K}_{\mathrm{df}, 3}$ will not be exact. This is shown in a particular example in Appendix $\mathrm{F}^{12}$

\subsection{Derivation}

In this section we present a derivation of the quantization condition described in the previous section. Following Ref. [130, we obtain the spectrum from the poles in the finitevolume Minkowski-space correlator 13

$$
C_{L}(E, \vec{P}) \equiv \int_{L} d^{4} x e^{i\left(E x^{0}-\vec{P} \cdot \vec{x}\right)}\left\langle 0\left|\mathrm{~T} \sigma(x) \sigma^{\dagger}(0)\right| 0\right\rangle .
$$

Here $\mathrm{T}$ indicates time-ordering and $\sigma(x)$ is an interpolating field coupling to states with an odd number of particles. The Fourier transform, implemented via an integral over the finite spatial volume, restricts the states to have total energy $E$ and momentum $\vec{P}=2 \pi \vec{n}_{P} / L$.

The simplest choice for $\sigma(x)$ is a one-particle interpolating field, $\phi(x)$, since in the interacting theory this will couple to states with any odd number of particles. In a simulation, however, it is advantageous to use a choice with larger overlap to the three-particle states of interest. An example is

$$
\sigma(x)=\int_{L} d^{4} y d^{4} z f(y, z) \phi(x) \phi(x+y) \phi(x+z),
$$

with $f$ a smooth function with period $L$ in all directions.

At fixed $\left\{L, \vec{n}_{P}\right\}{ }^{14}$ the spectrum of our theory is the set of CM frame energies $E_{j}^{*}$, $j=1,2, \cdots$ for which $C_{L}\left(E_{j}, \vec{P}\right)$ has a pole, with $E_{j}=\left(E_{j}^{* 2}+\vec{P}^{2}\right)^{1 / 2}$. Our goal is thus to

\footnotetext{
${ }^{12} \mathrm{~A}$ similar issue arises with the two-particle quantization condition when one truncates the angular momentum expansion. The "lost" states involving higher angular momenta are recovered if one reintroduces the higher partial wave amplitudes but with infinitessimal strength. The quantization condition then has solutions corresponding to free two-particle states projected onto states in appropriate irreps of the finitevolume symmetry group.

${ }^{13}$ Minkowski time turns out to be convenient for our analysis, even though numerical lattice determinations of the spectrum work in Euclidean time. The point is that the finite-volume spectrum is the same, however it is determined.

${ }^{14}$ It is more natural to think in terms of $\left\{L, \vec{n}_{P}\right\}$ rather than $\{L, \vec{P}\}$, since $\vec{n}_{P}$ is quantized whereas $\vec{P}$ varies with $L$.
} 
include all contributions to $C_{L}$ which fall at most like a power of $1 / L$, and determine the pole structure. In the previous section we summarized the main result of this work, but made no reference to the correlator in doing so. The connection is given by the following identity, the demonstration of which is the task of this section:

$$
C_{L}(E, \vec{P})=C_{\infty}(E, \vec{P})+i A^{\prime} \frac{1}{1+F_{3} \mathcal{K}_{\mathrm{df}, 3}} F_{3} A
$$

This result is valid up to terms exponentially suppressed in the volume, terms which we will discard implicitly throughout this section. The quantities $A^{\prime} \equiv A_{k^{\prime}, \ell^{\prime}, m^{\prime}}^{\prime}$ and $A \equiv A_{k, \ell, m}$ are, respectively, row and column vectors in [finite-volume momentum] $\times[$ two-particle angular momentum] space. Since $A$ and $A^{\prime}$ do not enter the quantization condition, we have not given their definitions above. Indeed, we think it most useful to introduce their definitions as they emerge in our all orders summation. We have also introduced $C_{\infty}$, which is an infinitevolume correlator whose definition we will also build up over the following subsections.

A key technical issue in the derivation is the need to use a non-standard pole prescription when definining momentum integrals in infinite-volume Feynman diagrams. This is at the root of the complications in definining $A^{\prime}, A$ and $C_{\infty}$. Despite these complications, it is possible to show that these quantities have the key property of having no poles, but only branch cuts ${ }^{15}$ It follows that, at fixed $\left\{L, \vec{n}_{P}\right\}, C_{L}$ diverges at all energies for which the matrix between $A$ and $A^{\prime}$ has a divergent eigenvalue. In addition, as long as $\mathcal{K}_{\mathrm{df}, 3}$ is nonzero, diverging eigenvalues of $F_{3}$ leave the finite-volume correlator finite. The spectrum is therefore given by energies for which $\left[1+F_{3} \mathcal{K}_{\mathrm{df}, 3}\right]$ has a vanishing eigenvalue, which is the quantization condition quoted above.

The demonstration of Eq. 5.42 proceeds by an all-orders analysis of the Feynman diagrams building up the correlator. As we accommodate any scalar field theory (assuming only a $\mathbb{Z}_{2}$ symmetry), Feynman diagrams consist of any number of even-legged vertices, as well as one each of the interpolating fields $\sigma$ and $\sigma^{\dagger}$, connected by propagators. The

\footnotetext{
${ }^{15}$ More precisely, we show that all diagrams which contribute to $A^{\prime}, A$ and $C_{\infty}$ have no poles. In certain cases, however, poles can arise from the all orders summation. Such poles always appear in $A^{\prime}, A, C_{\infty}$ and $\mathcal{K}_{\mathrm{df}, 3}$ in such away as to cancel each other, so that $C_{L}$ remains finite. We explain this in detail after completing the derivation, at the very end of this section.
} 
finite-volume condition enters here only through the prescription of summing (rather than integrating) the spatial components of all loop momenta

$$
\frac{1}{L^{3}} \sum_{\vec{q}=2 \pi \vec{n} / L} \int \frac{d q^{0}}{2 \pi} \quad \text { over all } \quad \vec{n} \in \mathbb{Z}^{3} .
$$

We now introduce the crucial observation that makes our derivation possible: Power-law finite-volume effects only enter through on-shell intermediate states. This motivates a reorganization of the sum of diagrams into a skeleton expansion that keeps all on-shell intermediate states explicit, while grouping off-shell states into Bethe-Salpeter kernels. Heuristically, the importance of on-shell intermediate states can be understood by noting that on-shell particles can travel arbitrarily far, and are thus maximally affected by the periodic boundary conditions. By contrast, off-shell states are localized so that the effect of finite-volume is smaller (and, indeed, exponentially suppressed in general).

The technical justification for this description begins by noting that the difference between a sum and an integral acting on a smooth (i.e. infinitely differentiable) function $f(\vec{q})$ falls off faster than any power of $1 / L[128]]^{16}$ As noted above, we treat terms with this highly suppressed scaling in $L$ as negligible, and thus set

$$
\left[\frac{1}{L^{3}} \sum_{\vec{q}}-\int_{\vec{q}}\right] f(\vec{q})=0 .
$$

By contrast, if a function $d(\vec{q})$ is not continuous but instead diverges for some real $\vec{q}$, or if some derivative diverges, then the sum-integral difference receives power-law corrections

$$
\left[\frac{1}{L^{3}} \sum_{\vec{q}}-\int_{\vec{q}}\right] d(\vec{q})=\mathcal{O}\left(L^{-n}\right),
$$

for some positive integer $n$. We keep all such contributions.

A convenient tool to determine when the summands of Feynman diagrams are singular is time-ordered perturbation theory (TOPT) ${ }^{17}$ In this method one first does all $k^{0}$ integrals, leaving only the sums over spatial components of loop momenta. (In a continuum application these would, of course, be replaced by integrals.) Each Feynman diagram then becomes a

\footnotetext{
${ }^{16}$ This is what we refer to as exponentially suppressed, although strictly it is not equivalent.

${ }^{17}$ For a clear discussion of this method see Chapter 13 of Ref. [247].
} 
sum of terms corresponding to the different time-orderings of the vertices. Within a given time ordering, each pair of neighboring vertices leads to an energy denominator,

$$
\frac{1}{E_{\text {cut }}-\sum_{y \in \text { cut }} \omega_{y}} \text {. }
$$

Here $E_{\text {cut }}$ is total energy flowing through the propagators in the "cut", which is the vertical line between adjacent vertices. The propagators have momenta $\vec{p}_{y}$ and on-shell energies $\omega_{y}$. For our correlator $E_{\text {cut }}$ can be $E, 0$ or $-E$, depending on the time ordering. All other factors in the summand are non-singular: they arise from momentum dependence in the vertices or from $1 / \omega$ factors.

Given the assumed $Z_{2}$ symmetry and our choices of $\sigma$ and $\sigma^{\dagger}$, the cuts in the diagrams contributing to $C_{L}$ can only involve an odd number of particles. Furthermore, given the restriction $m<E^{*}<5 m$, the only energy denominators which can vanish must involve three particles in the cut, i.e.

$$
\frac{1}{E-\omega_{k}-\omega_{a}-\omega_{k a}}
$$

Thus it is only when a three-particle state goes on shell that replacing the sum over spatial momenta with an integral can lead to power-law corrections.

The only subtlety in the application of this result to our analysis is that $m$ (which appears in $\omega_{k}^{2}=\vec{k}^{2}+m^{2}$ and in the condition on $E^{*}$ ) should be the physical and not the bare mass. Technically this arises because the usual geometric sum of irreducible two-point correlation functions shifts the pole position in the dressed propagator to the physical mass. This sum should be done before applying the TOPT analysis 18

We can now describe the skeleton expansion we use for $C_{L}$, which is displayed in Fig. 5.4 . Since only three-particle intermediate states can go on-shell, we display them explicitly, and

\footnotetext{
${ }^{18}$ Doing things in this order makes the application of TOPT more complicated, because the dressed propagator itself now has multiple-particle poles. This subtlety does not affect our analysis because all we are taking from TOPT is the conclusion that divergences occur when a time integral extends, undamped, over an infinite range. Thus it is the long-time dependence of the dressed propagator that matters, and this has the same form as that that of a free propagator but with the physical mass. We stress that we use TOPT only to identify diagrams that can lead to power-law corrections. We do not use TOPT to do the calculation, but rather use the standard relativistic Feynman rules.
} 
use a notation indicating that their momenta are summed. Intermediate states with five or more particles cannot go on shell for our range of $E^{*}$, and so sums over the momenta of such intermediate states can be replaced by integrals 19 These contributions can be grouped into infinite-volume Bethe-Salpeter kernels, which are defined below.

Each diagram in the expansion contains "endcaps" $\tilde{\sigma}^{\dagger}$ and $\widetilde{\sigma}$ on the far left and far right, respectively. These are each functions of the off-shell momenta of three attached propagators, subject to the constraint that they total $(E, \vec{P})$. Thus they can be written $\widetilde{\sigma}=\widetilde{\sigma}(q, p)$ and $\widetilde{\sigma}^{\dagger}=\widetilde{\sigma}^{\dagger}(q, p)$. For the example of the $\sigma$ operator given in Eq. (5.41),

$\widetilde{\sigma}(q, p)=\widetilde{f}(q, p)+\widetilde{f}(p, P-p-q)+\widetilde{f}(P-p-q, q)+\widetilde{f}(p, q)+\widetilde{f}(P-p-q, p)+\widetilde{f}(q, P-p-q)$,

where

$$
\widetilde{f}(q, p)=\int_{L} d^{4} x d^{4} y e^{i p x+i q y} f(x, y) .
$$

Note here that we use the mostly-minus metric, $p x=p^{0} x^{0}-\vec{p} \cdot \vec{x}$. The exact forms of $\widetilde{\sigma}$ and $\tilde{\sigma}^{\prime}$ not important to the final answer. We only require that they are analytic in the complex $q^{0}$ and $p^{0}$ planes and fall off fast enough at infinity to justify the contour integrals we perform below.

Between the endcaps, each diagram contains some number of two-to-two and three-tothree Bethe-Salpeter kernels. The two-to-two Bethe-Salpeter kernel $i B_{2}$ was introduced in Ref. [128]. It is the sum of all four-point diagrams (with external propagators amputated) that are two-particle irreducible in the $s$-channel, see Fig. 5.5 3 . Thus this kernel is the sum of all diagrams which have no on-shell intermediate states when the total CM energy being fed into the kernel is below $4 m$. Because $i B_{2}$ contains no on-shell intermediate states, the summands of all contributing terms are smooth functions of summed momenta. It follows that finite-volume corrections are exponentially suppressed and for our purposes negligible. We thus work from now on with the infinite-volume version of the kernel.

Similarly, $i B_{3}$ contains no diagram in which three propagators carry the total energy

\footnotetext{
${ }^{19}$ Here we are using the language of TOPT although we are calculating using relativistic propagators in which multiple time-orderings are contained within a single diagram. If we focus on a particular cut, however, then there is only one time ordering in which all particles can go on-shell.
} 


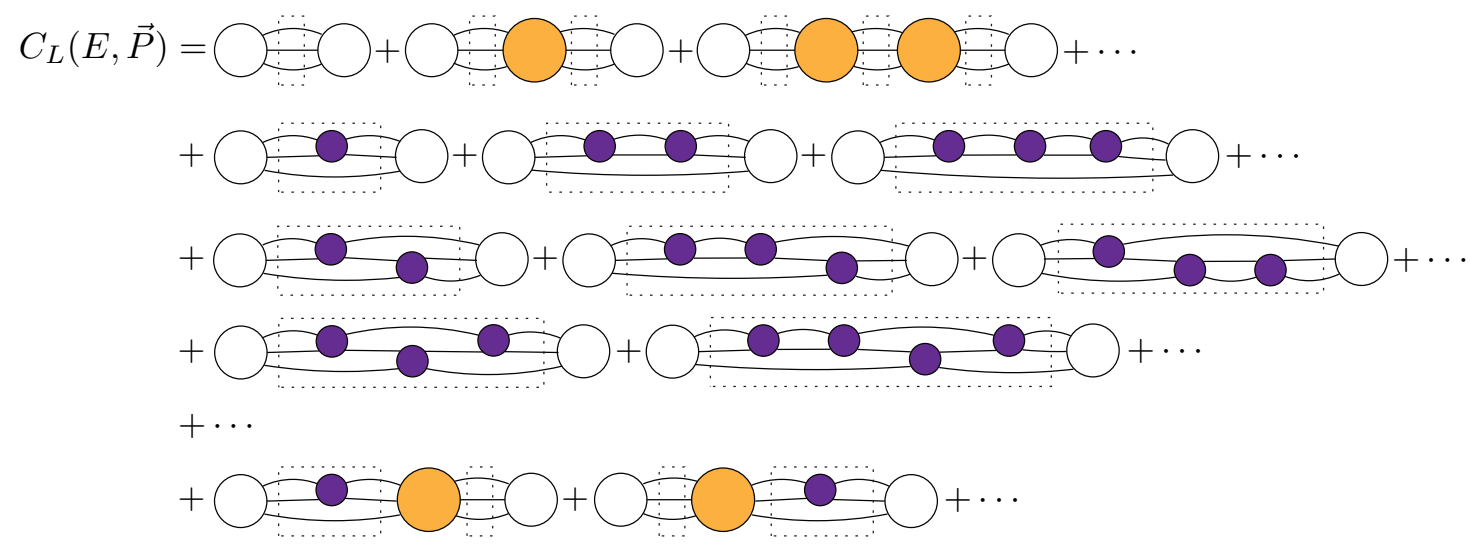

Figure 5.4: Skeleton expansion defining the finite-volume correlator. The rightmost blob in all diagrams represents a function of momentum $\tilde{\sigma}^{\dagger}$, whose specific form is determined by the interpolating fields defining the correlator. The leftmost blob represents an analogous function, $\widetilde{\sigma}$. Any insertion between these with four legs represents a two-to-two BetheSalpeter kernel $i B_{2}$. Any insertion with six legs represents an analogous three-to-three kernel $i B_{3}$. All lines connecting kernels and $\widetilde{\sigma}$-functions represent fully-dressed propagators. The dashed rectangles indicate that all loop momenta are summed rather than integrated, due to the finite-volume condition. The regions bounded by these rectangles also emphasize chains of loops that have common coordinates which prevent the diagram from factorizing. This is one of the central complications faced in this work. (For example the top line, with only three-to-three insertions, does factorize and is therefore a straightforward generalization of the two particle case.) 
a)

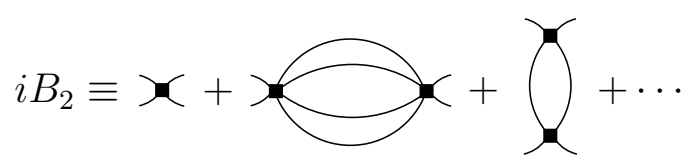

b)

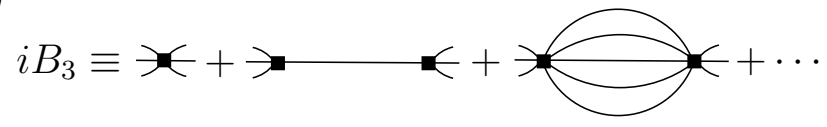

Figure 5.5: Examples of Feynmann diagrams contributing to (a) $i B_{2}$, the two-to-two BetheSalpeter kernel and (b) $i B_{3}$, the analogous three-to-three kernel.

and momentum $(E, \vec{P})$. Diagrams with one propagator carrying the total energy and momentum as well as any odd number greater than three are allowed, see Figure 5.5b. The technical definition of this quantity is slightly more complicated because of the possibility of having single-particle intermediate states. To give the definition, we first introduce three intermediate quantities $i \widetilde{B}_{3 \rightarrow 3}$, $i \widetilde{B}_{1 \rightarrow 3}$, i $\widetilde{B}_{3 \rightarrow 1}$. In each case $i \widetilde{B}_{n \rightarrow m}$ is the sum of all amputated diagrams, with $n$ incoming and $m$ outgoing external lines, which are threeparticle irreducible in the $s$-channel. Next we introduce a modified, fully-dressed propagator $\widetilde{\Delta}(q)$. This differs from the standard propagator, defined in Eq. 5.51 below, only in that its self-energy graphs are three-particle irreducible (as opposed to the usual one-particle irreducible). In terms of these ingredients, our three-to-three kernel is

$$
i B_{3} \equiv i \widetilde{B}_{3 \rightarrow 3}+i \widetilde{B}_{3 \rightarrow 1} \widetilde{\Delta} i \widetilde{B}_{1 \rightarrow 3}
$$

In direct analogy to the two-to-two case, $i B_{3}$ is the sum of all diagrams with no on-shell intermediate states when the CM energy is between $m$ and $5 m$. Again we drop exponentially suppressed corrections and work with the infinite-volume version of the kernel.

We stress that the need for two kinds of kernels follows directly from requiring that both $i B_{2}$ and $i B_{3}$ contain only connected diagrams. For example, one might think that only the top line of Fig. 5.4 is needed, as long as one chooses an alternative $i B_{3}$ which accomodates pairwise scatterings. This is attractive since the top line closely resembles the two-particle skeleton expansion of Ref. [130], in which the correlator is written as a ladder series of two- 
particle loops. However, in the three-particle sector this approach results in $i B_{3}$ containing Dirac delta functions, which is a problem because we rely on smoothness of the kernel in our derivation. For this reason the three-particle case is fundamentally different. After much investigation, we found it most convenient to require that $i B_{3}$ only contain connected diagrams and thus display all pairwise scatterings explicitly.

Finally, in our skeleton expansion all kernels and interpolating functions are connected by fully-dressed propagators,

$$
\Delta(q) \equiv \int d^{4} x e^{i q \cdot x}\langle 0|\mathrm{~T} \phi(x) \phi(0)| 0\rangle .
$$

Here $\phi(x)$ is a one particle interpolating field defined with on-shell renormalization

$$
\lim _{q^{0} \rightarrow \omega_{q}} \Delta(q)\left[\left(q^{2}-m^{2}\right) / i\right]=1 .
$$

Since we are working with fully dressed propagators, we do not include self-energy contributions explicitly in our skeleton expansion. We use infinite-volume fully-dressed propagators throughout, which is justified because the self-energy graphs do not contain on-shell intermediate states.

In summary, the skeleton expansion of Fig. 5.4 displays explicitly all the intermediate states that can go on shell and give rise to power-law corrections. All intermediate states which cannot go on-shell are included in the infinite-volume two-to-two and three-to-three Bethe-Salpeter kernels.

In the remaining subsections, we work through the different classes of diagrams appearing in this expansion. First, in Sec. 5.3.1, we sum diagrams containing only $i B_{2}$ kernels on the same pair of propagators (second line of Figure 5.4). Then, in Secs. 5.3.2 and 5.3.3, we sum diagrams with, respectively, one or two changes in the pair that is being scattered (third and fourth lines of Figure 5.4). At this stage, we can extend the pattern and sum all diagrams built from $i B_{2}$ kernels with any number of changes in the scattered pair. This is done in Sec. 5.3.4. Incorporating three-to-three insertions at this point is relatively easy, and is done in Sec. 5.3.4, leading to the final result for $C_{L}$ given in Eq. (5.42).

As we proceed we identify the diagrams contributing to $\mathcal{K}_{2}$ and $\mathcal{K}_{\mathrm{df}, 3}$, as well as $A, A^{\prime}$ and $C_{\infty}$. The precise definitions of these infinite-volume quantities will thus emerge step by step. 


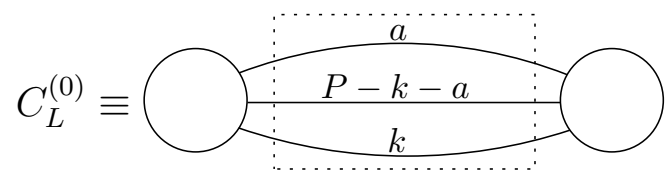

Figure 5.6: Finite-volume correlator diagram with no kernel insertions.

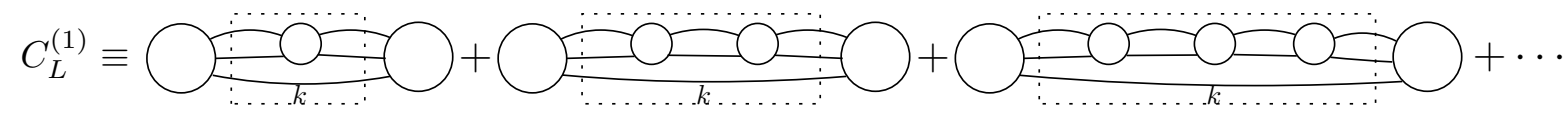

Figure 5.7: Subset of finite-volume correlator diagrams containing only two-to-two insertions, with no change in the scattered pair.

\subsubsection{Two-to-two insertions: no switches}

In this section we sum the diagrams of Figs. 5.6 and 5.7. Each diagram contains only $B_{2}$ insertions, all of which scatter the same pair of propagators. We separate the diagram with no $B_{2}$ insertions, labeled $C_{L}^{(0)}$ [Fig. 5.6. from the sum of diagrams with one or more insertion, denoted $C_{L}^{(1)}$ [Fig. 5.7]. We refer to these diagrams as having "no switches", meaning that the pair that is scattered does not change. This designation anticipates subsequent sections in which we sum diagrams with one or more switches in the scattered pair.

An important check on the calculation of this subsection is obtained by noting that the no-switch diagrams are the complete set appearing in a theory of two different particle types, with one of the types non-interacting. This is the case provided that the correlator is constructed with fields that interpolate one free particle and two interacting particles. Thus the result for $C_{L}^{(0)}+C_{L}^{(1)}$ must be that for the full finite-volume correlator in the two-plus-spectator theory. This check is discussed below.

We begin our detailed calculation by determining the finite-volume residue of the no- 
insertion diagram of Fig. 5.6. This diagram represents the expression 20

$$
C_{L}^{(0)} \equiv \frac{1}{6} \frac{1}{L^{6}} \sum_{\vec{k}, \vec{a}} \int \frac{d a^{0}}{2 \pi} \int \frac{d k^{0}}{2 \pi} \sigma(k, a) \Delta(k) \Delta(a) \Delta(P-k-a) \sigma^{\dagger}(k, a),
$$

where the $1 / 6$ is the symmetry factor. We stress that the $\Delta$ 's are fully dressed propagators, with the normalization given in Eq. (5.52).

We first evaluate the $a^{0}$ and $k^{0}$ integrals using contour integration, wrapping both contours in the lower half of the respective complex planes. Each contour encircles a one-particle pole $\left[a^{0}=\omega_{a}-i \epsilon\right.$ and $\left.k^{0}=\omega_{k}-i \epsilon\right]$ as well as three-particle (and higher) poles from excitedstate contributions to the propagators. The result of integration may thus be written

$$
C_{L}^{(0)}=\frac{1}{6} \frac{1}{L^{6}} \sum_{\vec{k}, \vec{a}}\left[\frac{\sigma\left(\left[\omega_{k}, \vec{k}\right],\left[\omega_{a}, \vec{a}\right]\right) \Delta(P-k-a) \sigma^{\dagger}\left(\left[\omega_{k}, \vec{k}\right],\left[\omega_{a}, \vec{a}\right]\right)}{2 \omega_{k} 2 \omega_{a}}+\mathcal{R}(\vec{k}, \vec{a})\right],
$$

where $\mathcal{R}(\vec{k}, \vec{a})$ is the contribution from excited-state poles. Here $k$ and $a$ appearing in $\Delta(P-k-a)$ are now understood as on-shell four-vectors, a fact that we have made explicit in the arguments of $\sigma$ and $\sigma^{\dagger}$. We next note that $\Delta(P-k-a)$ can be split into its one-particle pole plus a remainder:

$$
\Delta(P-k-a)=\frac{i}{2 \omega_{k a}\left(E-\omega_{k}-\omega_{a}-\omega_{k a}\right)}+r(\vec{k}, \vec{a}) .
$$

Substituting Eq. (5.55) into Eq. (5.54) gives

$$
C_{L}^{(0)}=\frac{1}{6} \frac{1}{L^{6}} \sum_{\vec{k}, \vec{a}}\left[\frac{i \sigma\left(\left[\omega_{k}, \vec{k}\right],\left[\omega_{a}, \vec{a}\right]\right) \sigma^{\dagger}\left(\left[\omega_{k}, \vec{k}\right],\left[\omega_{a}, \vec{a}\right]\right)}{2 \omega_{k} 2 \omega_{a} 2 \omega_{k a}\left(E-\omega_{k}-\omega_{a}-\omega_{k a}\right)}+\mathcal{R}^{\prime}(\vec{k}, \vec{a})\right],
$$

where $\mathcal{R}^{\prime}$ is the sum of $\mathcal{R}$ and the term containing $r$. This grouping is convenient because $\mathcal{R}^{\prime}(\vec{k}, \vec{a})$ is a smooth function of $\vec{k}$ and $\vec{a}$ for our range of $E$, since we have explicitly pulled out the three-particle singularity. Indeed, we are free to further adjust the separation between first and second terms, as long as the latter remains smooth. For the following development we need to include the damping function $H(\vec{k})$ in the singular term. We recall that $H(\vec{k})$, defined in Eqs. (5.27) and (5.28), is a smooth function which equals unity when the other

\footnotetext{
${ }^{20}$ In the remainder of this article we drop tilde's on the Fourier-transformed interpolating operators, $\tilde{\sigma}(k, a)$ and $\tilde{\sigma}^{\dagger}(k, a)$, since we no longer use the position-space forms.
} 
two particles (those with momenta $a$ and $P-k-a$ ) are kinematically allowed to be on shell (for the given values of $E$ and $\vec{k}$ ). In particular, if we multiply the singular term by $1=H(\vec{k})+[1-H(\vec{k})]$, then the $1-H(\vec{k})$ term cancels the singularity, leading to a smooth function that can be added to $\mathcal{R}^{\prime}$ to obtain a new residue $\mathcal{R}^{\prime \prime}$ :

$$
C_{L}^{(0)}=\frac{1}{6} \frac{1}{L^{6}} \sum_{\vec{k}, \vec{a}}\left[\frac{i \sigma\left(\left[\omega_{k}, \vec{k}\right],\left[\omega_{a}, \vec{a}\right]\right) \sigma^{\dagger}\left(\left[\omega_{k}, \vec{k}\right],\left[\omega_{a}, \vec{a}\right]\right) H(\vec{k})}{2 \omega_{k} 2 \omega_{a} 2 \omega_{k a}\left(E-\omega_{k}-\omega_{a}-\omega_{k a}\right)}+\mathcal{R}^{\prime \prime}(\vec{k}, \vec{a})\right] .
$$

At this stage we want to rewrite $C_{L}^{(0)}$ as an infinite-volume ( $L$-independent) quantity plus a remainder. Infinite-volume quantities differ only in that loop-momenta are integrated rather than summed. We can thus pull out the infinite-volume object by replacing each sum with an integral plus a sum-integral difference. We stress that integrals, unlike sums, require a pole-prescription. We are free to use any prescription we like, and it turns out to be most convenient to make a non-standard choice which we call the "modified principle-value" or $\widetilde{\mathrm{PV}}$ prescription. This is defined in the present context as follow 21

$$
\begin{aligned}
& \frac{1}{2} \widetilde{\mathrm{PV}} \int_{\vec{a}} \frac{i \sigma\left(\left[\omega_{k}, \vec{k}\right],\left[\omega_{a}, \vec{a}\right]\right) \sigma^{\dagger}\left(\left[\omega_{k}, \vec{k}\right],\left[\omega_{a}, \vec{a}\right]\right) H(\vec{k})}{2 \omega_{a} 2 \omega_{k a}\left(E-\omega_{k}-\omega_{a}-\omega_{k a}\right)} \\
& \quad \equiv \frac{1}{2} \int_{\vec{a}} \frac{i \sigma\left(\left[\omega_{k}, \vec{k}\right],\left[\omega_{a}, \vec{a}\right]\right) \sigma^{\dagger}\left(\left[\omega_{k}, \vec{k}\right],\left[\omega_{a}, \vec{a}\right]\right) H(\vec{k})}{2 \omega_{a} 2 \omega_{k a}\left(E-\omega_{k}-\omega_{a}-\omega_{k a}+i \epsilon\right)}-\sigma_{\ell^{\prime}, m^{\prime}}^{*}(\vec{k}) i \rho_{\ell^{\prime}, m^{\prime} ; \ell, m}(\vec{k}) \sigma_{\ell, m}^{\dagger *}(\vec{k})
\end{aligned}
$$

where

$$
\begin{aligned}
\rho_{\ell^{\prime}, m^{\prime} ; \ell, m}(\vec{k}) & \equiv \delta_{\ell^{\prime}, \ell} \delta_{m^{\prime}, m} H(\vec{k}) \widetilde{\rho}\left(P_{2}\right) \\
\widetilde{\rho}\left(P_{2}\right) & \equiv \frac{1}{16 \pi \sqrt{P_{2}^{2}}} \times \begin{cases}-i \sqrt{P_{2}^{2} / 4-m^{2}} & (2 m)^{2}<P_{2}^{2}, \\
\left|\sqrt{P_{2}^{2} / 4-m^{2}}\right| & P_{2}^{2} \leq(2 m)^{2}\end{cases}
\end{aligned}
$$

was introduced in Eq. 5.25 above. Here $P_{2}=\left(E-\omega_{k}, \vec{P}-\vec{k}\right)$ is the four-momentum flowing through the upper two particles. Recall also that $E_{2, k}^{* 2}=P_{2}^{2}$, and $q_{k}^{*}=\sqrt{P_{2}^{2} / 4-m^{2}}$.

\footnotetext{
${ }^{21}$ In the definition of $\widetilde{\mathrm{PV}}$ we are using $\sigma$ and $\sigma^{\dagger}$ which are continuous functions of $\vec{a}$ and $\vec{k}$. Since these were originally defined only for discrete finite-volume momenta, this requires a continuation of the original functions. We require only that the continuation is smooth and slowly varying. More precisely we demand

$$
\left[\frac{1}{L^{3}} \sum_{\vec{a}}-\int_{\vec{a}}\right] \sigma\left(\left[\omega_{k}, \vec{k}\right],\left[\omega_{a}, \vec{a}\right]\right)=\mathcal{O}\left(e^{-m L}\right)
$$


To complete the definition we need to explain the meanings of the on-shell quantities $\sigma_{\ell^{\prime}, m^{\prime}}^{*}(\vec{k})$ and $\sigma_{\ell, m}^{\dagger *}(\vec{k})$. Similar quantities will appear many times below so we give here a detailed description. First recall that $\left(\omega_{a}^{*}, \vec{a}^{*}\right)$ is the four-vector obtained by boosting $\left(\omega_{a}, \vec{a}\right)$ with velocity $\vec{\beta}_{k}=-(\vec{P}-\vec{k}) /\left(E-\omega_{k}\right)$. This boost is only physical if $E_{2, k}^{*}>0$, a constraint which is guaranteed to be satisfied by the presence of $H(\vec{k})$ in Eq. 5.59 . We now change variables from $\vec{a}$ to $\vec{a}^{*}$ and define

$$
\sigma^{*}\left(\vec{k}, \vec{a}^{*}\right) \equiv \sigma\left(\left[\omega_{k}, \vec{k}\right],\left[\omega_{a}, \vec{a}\right]\right)
$$

and similarly for $\sigma^{\dagger}$. The left-hand side exemplifies our general notation that, if the momentum argument is a three-vector, e.g. $\vec{k}$, then the momentum is on-shell, e.g. $k^{0}=\omega_{k}$. If the argument is a four-momentum, e.g. $k$, then it is, in general, off shell. Here we include a superscript $*$ on $\sigma$ to indicate that it is strictly a different function from that appearing in say Eq. (5.57), since it depends on different coordinates (in particular on momenta defined in different frames). Next we decompose $\sigma^{*}$ and $\sigma^{\dagger *}$ into spherical harmonics in the CM frame

$$
\begin{aligned}
\sigma^{*}\left(\vec{k}, \vec{a}^{*}\right) & \equiv \sqrt{4 \pi} Y_{\ell, m}\left(\hat{a}^{*}\right) \sigma_{\ell, m}^{*}\left(\vec{k}, a^{*}\right) \\
\sigma^{\dagger *}\left(\vec{k}, \vec{a}^{*}\right) & \equiv \sqrt{4 \pi} Y_{\ell, m}^{*}\left(\hat{a}^{*}\right) \sigma_{\ell, m}^{\dagger *}\left(\vec{k}, a^{*}\right),
\end{aligned}
$$

where there is an implicit sum over $\ell$ and $m$. Our convention, used throughout, is that the quantities to the left of the three particle "cut" are decomposed using $Y_{\ell, m}$ 's while those to the right use the complex conjugate harmonics. Finally, with the "starred" quantities in hand we can define on-shell restrictions. As explained in the introduction, $P-k-a$ is only on shell if $a^{*}=q_{k}^{*}$, so we define

$$
\sigma_{\ell, m}^{*}(\vec{k}) \equiv \sigma_{\ell, m}^{*}\left(\vec{k}, q_{k}^{*}\right), \quad \sigma_{\ell, m}^{\dagger *}(\vec{k}) \equiv \sigma_{\ell, m}^{\dagger *}\left(\vec{k}, q_{k}^{*}\right)
$$

These are the quantities appearing in the " $\rho$ " term in Eq. (5.59p. If $E_{2, k}^{*}<2 m$, then the $\vec{a}, \vec{b}_{k a}$ pair are below threshold, and $\sigma_{\ell, m}^{*}$ and $\sigma_{\ell, m}^{\dagger *}$ must be obtained by analytic continuation from above threshold.

The reason for using this rather elaborate pole prescription is that we want the integral over $\vec{a}$ to produce a smooth function of $\vec{k}$. This allows the sum over $\vec{k}$ to be replaced by 
an integral. If we were to instead use the $i \epsilon$ prescription, then the resulting function of $\vec{k}$ would have a unitary cusp at $E_{2, k}^{*}=2 \mathrm{~m}$. This observation leads us to consider a principalvalue pole prescription instead. Recall that $\rho$ is defined so that, for $E_{2, k}^{*}>2 m$, Eq. 5.59 simply gives the standard principal-value prescription. It turns out that this choice does give a smooth function of $\vec{k}$, provided that one uses analytic continuation to extend from $E_{2, k}^{*}>2 m$ to $E_{2, k}^{*}<2 m$. This is accomplished by our subthreshold definition of $\rho$, which is then smoothly turned off by the function $H(\vec{k})$. A derivation of the smoothness property is given in Appendix E. We stress that the $\widetilde{\mathrm{PV}}$ prescription is always defined relative to a spectator momentum, here $\vec{k}$.

A slightly more general form of the $\widetilde{\mathrm{PV}}$ prescription is instructive and will be useful below. For any two-particle four-momentum $P_{2}$ for which the only kinematically allowed cut involves two particles, we can write

$$
\begin{aligned}
\widetilde{\mathrm{PV}} \int_{a} A\left(P_{2}, a\right) B\left(P_{2}, a\right) \Delta(a) \Delta\left(P_{2}-a\right)=\int_{a} A\left(P_{2}, a\right) B\left(P_{2}, a\right) \Delta(a) \Delta\left(P_{2}-a\right) \\
-\left.2 i J\left(P_{2}^{2} /\left[4 m^{2}\right]\right) \widetilde{\rho}\left(P_{2}\right)\left[\int_{\hat{a}^{*}} A^{*}\left(P_{2}, \vec{a}^{*}\right) B^{*}\left(P_{2}, \vec{a}^{*}\right)\right]\right|_{a^{*}=\sqrt{P_{2}^{2} / 4-m^{2}}} .
\end{aligned}
$$

Here $A$ and $B$ are smooth, non-singular functions of their arguments. The quantities $A^{*}$ and $B^{*}$ are defined in a similar way to $\sigma^{*}$ above, e.g. $A^{*}\left(P_{2}, \vec{a}^{*}\right)=A\left(P_{2},\left[\omega_{a}, \vec{a}\right]\right)$, where the boost to the two-particle CM has velocity $-\vec{P}_{2} / P_{2}^{0}$. The function $J$, defined in Eq. 5.29, ensures that this boost is well defined ${ }^{22}$ Finally, the angular integral is normalized such that $\int_{\hat{a}^{*}} 1=1$. The form 5.66 makes clear that the prescription can be defined for fourmomentum integrals (and not just three-momentum integrals) and that its dependence on external momenta enters entirely through $P_{2}$. We have also used the angular independence of $\rho$ to rewrite the subtraction term as an angular average in the CM frame. The two functions $A$ and $B$ could be combined into one, but are left separate since in our applications we always have separate functions to the left and right of the cut.

Returning to the main argument, we now substitute

$$
\frac{1}{L^{3}} \sum_{\vec{a}}=\widetilde{\mathrm{PV}} \int_{\vec{a}}+\left[\frac{1}{L^{3}} \sum_{\vec{a}}-\widetilde{\mathrm{PV}} \int_{\vec{a}}\right]
$$

\footnotetext{
${ }^{22}$ Here $J$ is playing the role of $H(\vec{k})=J\left(P_{2}^{2} /\left[4 m^{2}\right]\right)$ in Eq. 5.59 .
} 
into Eq. (5.57) to reach

$$
\begin{aligned}
C_{L}^{(0)}=\frac{1}{6} \frac{1}{L^{3}} \sum_{\vec{k}} \widetilde{\mathrm{PV}} \int_{\vec{a}}\left[\frac{i \sigma\left(\left[\omega_{k}, \vec{k}\right],\left[\omega_{a}, \vec{a}\right]\right) \sigma^{\dagger}\left(\left[\omega_{k}, \vec{k}\right],\left[\omega_{a}, \vec{a}\right]\right) H(\vec{k})}{2 \omega_{k} 2 \omega_{a} 2 \omega_{k a}\left(E-\omega_{k}-\omega_{a}-\omega_{k a}\right)}+\mathcal{R}^{\prime \prime}(\vec{k}, \vec{a})\right] \\
+\frac{1}{6} \frac{1}{L^{3}} \sum_{\vec{k}}\left[\frac{1}{L^{3}} \sum_{\vec{a}}-\widetilde{\mathrm{PV}} \int_{\vec{a}}\right] \frac{i \sigma\left(\left[\omega_{k}, \vec{k}\right],\left[\omega_{a}, \vec{a}\right]\right) \sigma^{\dagger}\left(\left[\omega_{k}, \vec{k}\right],\left[\omega_{a}, \vec{a}\right]\right) H(\vec{k})}{2 \omega_{k} 2 \omega_{a} 2 \omega_{k a}\left(E-\omega_{k}-\omega_{a}-\omega_{k a}\right)}
\end{aligned}
$$

Note that the sum-integral-difference operator annihilates $\mathcal{R}^{\prime \prime}(\vec{k}, \vec{a})$ up to exponentially suppressed terms. As already noted, we can replace the sum over $\vec{k}$ with an integral in the first term, resulting in the infinite-volume quantity

$$
C_{\infty}^{(0)} \equiv \frac{1}{6} \int_{\vec{k}} \widetilde{\mathrm{PV}} \int_{\vec{a}}\left[\frac{i \sigma\left(\left[\omega_{k}, \vec{k}\right],\left[\omega_{a}, \vec{a}\right]\right) \sigma^{\dagger}\left(\left[\omega_{k}, \vec{k}\right],\left[\omega_{a}, \vec{a}\right]\right) H(\vec{k})}{2 \omega_{k} 2 \omega_{a} 2 \omega_{k a}\left(E-\omega_{k}-\omega_{a}-\omega_{k a}\right)}+\mathcal{R}^{\prime \prime}(\vec{k}, \vec{a})\right]
$$

Note that no pole prescription is required for the $\vec{k}$ integral.

The second term in Eq. (5.68) is then the finite-volume residue. First we note that we can multiply the summand/integrand by $H(\vec{a}) H\left(\vec{b}_{k a}\right)$, since the remainder cancels the pole and thus has vanishing sum-integral difference. Next we use the identity for sum-integral differences presented in Eq. D.1 of Appendix D. This is based on an extension of the work of Ref. [130] to include the effects of subthreshold momenta and the $\widetilde{P V}$ prescription. The essence of the identity is that the sum-integral difference picks out the on-shell residue of the singularity multiplied by a kinematic function. In more detail the identity makes use of the analytic properties of $\sigma_{\ell, m}^{*}\left(\vec{k}, a^{*}\right)$ and $\sigma_{\ell, m}^{\dagger *}\left(\vec{k}, a^{*}\right)$, the functions defined in Eqs. 5.62 5.64 above. The result is that

$$
\begin{aligned}
C_{L}^{(0)} & =C_{\infty}^{(0)}+\frac{1}{L^{3}} \sum_{\vec{k}} \frac{1}{6 \omega_{k}} \sigma_{\ell^{\prime}, m^{\prime}}^{*}(\vec{k}) i F_{\ell^{\prime}, m^{\prime} ; \ell, m}(\vec{k}) \sigma_{\ell, m}^{\dagger *}(\vec{k}), \\
& =C_{\infty}^{(0)}+\sigma_{k^{\prime}, \ell^{\prime}, m^{\prime}}^{*} \frac{1}{6 \omega_{k} L^{3}} i F_{k^{\prime}, \ell^{\prime}, m^{\prime} ; k, \ell, m} \sigma_{k, \ell, m}^{\dagger *},
\end{aligned}
$$

where the finite-volume kinematical function is

$$
\begin{aligned}
& F_{\ell^{\prime}, m^{\prime} ; \ell, m}(\vec{k}) \equiv F_{\ell^{\prime}, m^{\prime} ; \ell, m}^{i \epsilon}(\vec{k})+\rho_{\ell^{\prime}, m^{\prime} ; \ell, m}(\vec{k}) \\
& F_{\ell^{\prime}, m^{\prime} ; \ell, m}^{i \epsilon}(\vec{k}) \equiv \frac{1}{2}\left[\frac{1}{L^{3}} \sum_{\vec{a}}-\int_{\vec{a}}\right] \frac{4 \pi Y_{\ell^{\prime}, m^{\prime}}\left(\hat{a}^{*}\right) Y_{\ell, m}^{*}\left(\hat{a}^{*}\right) H(\vec{k}) H(\vec{a}) H\left(\vec{b}_{k a}\right)}{2 \omega_{a} 2 \omega_{k a}\left(E-\omega_{k}-\omega_{a}-\omega_{k a}+i \epsilon\right)}\left(\frac{a^{*}}{q_{k}^{*}}\right)^{\ell+\ell^{\prime}}
\end{aligned}
$$




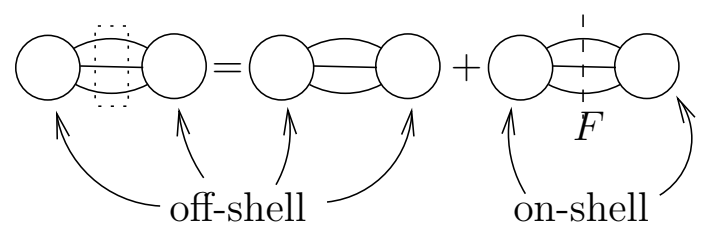

Figure 5.8: Diagrammatic representation of Eq. (5.75). This important identity is used throughout the derivation.

and

$$
\begin{gathered}
F_{k^{\prime}, \ell^{\prime}, m^{\prime} ; k, \ell, m} \equiv \delta_{k^{\prime}, k} F_{\ell^{\prime}, m^{\prime} ; \ell, m}(\vec{k}), \quad \sigma_{k, \ell, m}^{*} \equiv \sigma_{\ell, m}^{*}(\vec{k}) \\
\text { and } \sigma_{k, \ell, m}^{\dagger *} \equiv \sigma_{\ell, m}^{\dagger *}(\vec{k}) \text { for } \vec{k} \in(2 \pi / L) \mathbb{Z}^{3}
\end{gathered}
$$

are the restrictions of the on-shell functions to finite-volume momenta. These definitions are first given in Eqs. 5.22 5.24) above. All indices in Eq. (5.71) are understood to be summed, including $k$ and $k^{\prime}$ which are summed over the allowed values of finite-volume momenta. This index structure appears repeatedly in our derivation, and from now on we leave indices implicit. Indeed, using the matrix notation introduced in Section 5.1, we can write the final result compactly as

$$
C_{L}^{(0)}=C_{\infty}^{(0)}+\sigma^{*} \frac{i F}{6 \omega L^{3}} \sigma^{\dagger *}
$$

This is the main result of this subsection.

Our treatment of the 3-particle cut will be recyled repeatedly in the following, except that $\sigma$ and $\sigma^{\dagger}$ will be replaced by other smooth functions of the momenta. Since no properties of $\sigma$ and $\sigma^{\dagger}$ other than smoothness were used in the derivation of Eq. 5.75, the result generalizes immediately. It is useful to have a diagrammatic version, and this is given in Fig. 5.8. The key feature of the result is that the finite-volume residue depends only on onshell restrictions of the quantities appearing on either side of the cut (analytically continued below threshold as needed).

Before considering diagrams containing two-to-two insertions, we take stock of the impact of using the non-standard $\widetilde{\mathrm{PV}}$ pole prescription. First we relate $C_{\infty}^{(0)}$ [defined in 
Eq. (5.69)] to the conventional infinite-volume form which uses the $i \epsilon$ prescription. The latter is

$$
\begin{aligned}
C_{\infty}^{(0), i \epsilon} & \equiv \frac{1}{6} \int_{\vec{k}, \vec{a}}\left[\frac{i \sigma\left(\left[\omega_{k}, \vec{k}\right],\left[\omega_{a}, \vec{a}\right]\right) \sigma^{\dagger}\left(\left[\omega_{k}, \vec{k}\right],\left[\omega_{a}, \vec{a}\right]\right) H(\vec{k})}{2 \omega_{k} 2 \omega_{a} 2 \omega_{k a}\left(E-\omega_{k}-\omega_{a}-\omega_{k a}+i \epsilon\right)}+\mathcal{R}^{\prime \prime}(\vec{k}, \vec{a})\right], \\
& =\frac{1}{6} \int_{k, a} \sigma(k, a) \Delta(k) \Delta(a) \Delta(P-k-a) \sigma^{\dagger}(k, a),
\end{aligned}
$$

where $\int_{k} \equiv \int d^{4} k /(2 \pi)^{4}$, etc., indicate integrals over four-momenta. To obtain the second line, which is the standard expression for the Feynman diagram, we have reversed the steps leading from Eq. 5.53 to 5.57 . It then follows from the definition of the $\widetilde{\mathrm{PV}}$ prescription, Eq. (5.59), that

$$
C_{\infty}^{(0)}=C_{\infty}^{(0), i \epsilon}-\int_{\vec{k}} \sigma^{*}(\vec{k}) \frac{i \rho(\vec{k})}{6 \omega_{k}} \sigma^{\dagger *}(\vec{k}) .
$$

This relation is similar in form to Eq. (5.75), with the " $F$-cut" being replaced by a " $\rho$-cut". The key point for present purposes is that the $\rho$-cut term in Eq. (5.78) does not introduce poles as a function of $E$. This follows from noting that $\rho$ is a finite function of $(E, \vec{P})$ and $\vec{k}$, which has a finite range of support in the latter.

We can also determine the form of the finite-volume correction if we use the $i \epsilon$ prescription throughout, including in $F$ [see Eq. (5.73) above]. This connects our result to earlier work on two-particle quantization conditions, e.g. Ref. [130], where $F^{i \epsilon}$ was used. Defining

$$
F_{k^{\prime}, \ell^{\prime}, m^{\prime} ; k, \ell, m}^{i \epsilon} \equiv \delta_{k^{\prime}, k} F_{\ell^{\prime}, m^{\prime} ; \ell, m}^{i \epsilon}(\vec{k})
$$

it follows from Eq. 5.73 that

$$
F_{k^{\prime}, \ell^{\prime}, m^{\prime} ; k, \ell, m}=F_{k^{\prime}, \ell^{\prime}, m^{\prime} ; k, \ell, m}^{i \epsilon}+\delta_{k^{\prime}, k} \rho_{\ell^{\prime}, m^{\prime} ; \ell, m}(\vec{k}) .
$$

Combining the results above we then find

$$
C_{L}^{(0)}=C_{\infty}^{(0), i \epsilon}+\sigma \frac{i F^{i \epsilon}}{6 \omega L^{3}} \sigma^{\dagger}+\left[\frac{1}{L^{3}} \sum_{\vec{k}}-\int_{\vec{k}}\right] \sigma^{*}(\vec{k}) \frac{i \rho(\vec{k})}{6 \omega_{k}} \sigma^{\dagger *}(\vec{k})
$$

Thus we see that, were we to use quantities defined using the $i \epsilon$ prescription, we would need to account for the additional finite-volume correction coming from the last term, which arises due to the cusp at threshold 23 This extra term greatly complicates the all-orders

\footnotetext{
${ }^{23}$ This term is absent in the two-particle analysis, where there is only a single value of $\vec{k}$ (the total momentum flowing through the two-particle system).
} 
summation of diagrams. We have found it is more convenient to approach the analysis of finite-volume diagrams in two steps: first relate finite-volume quantities to $\widetilde{\mathrm{PV}}$-quantities (for example relating $C_{L}^{(0)}$ to $C_{\infty}^{(0)}$ ), and then relate $\widetilde{\mathrm{PV}}$-quantities to those defined with the standard $i \epsilon$ prescription $\left(C_{\infty}^{(0)}\right.$ to $\left.C_{\infty}^{(0), i \epsilon}\right)$. We concentrate on the first step in this article.

We now turn to diagrams of Fig. 5.7. We recall that only three-particle on-shell intermediate states lead to power-law finite-volume dependence. To isolate such terms we first do the $k^{0}$ integral and keep only the pole at $k^{0}=\omega_{k}$. Other poles will be collected into infinite-volume quantities, as for $C_{L}^{(0)}$. This means that we can replace $\Delta(k)$ with $1 /\left(2 \omega_{k}\right)$ and set $k^{0}=\omega_{k}$ in all finite-volume terms. Furthermore we can pull out the sum

$$
\frac{1}{L^{3}} \sum_{\vec{k}} \frac{1}{2 \omega_{k}}
$$

and consider the summand at fixed values of $\vec{k}$. The result of these steps is shown in Fig. 5.9a.

At each fixed value, we are left precisely with all scattering diagrams for two particles with energy-momentum $\left(E-\omega_{k}, \vec{P}-\vec{k}\right)$. We can thus follow the approach of Ref. [130] to obtain the answer for this set of diagrams. In particular, we can repeatedly use the sumintegral difference identity of Eq. (5.75) and Fig. 5.8 to replace sums over the two-particle loop momenta with integrals plus factors of $F$. As already noted, the identity holds if either $\sigma$ or $\sigma^{\dagger}$ (or both) are replaced by $B_{2}$. This substitution is also indicated in Fig. 5.9 a.

Our next step is to sum all diagrams into a convenient form by regrouping terms according to the number of $F$ insertions. This is depicted in Fig. $5.9 \mathrm{~b}$. We first consider terms with no $F$ insertions. These are conveniently combined with the smooth terms arising when the $k^{0}$ contour encircles higher-particle poles, yielding

$$
\begin{aligned}
C_{\infty}^{(1)}=\int_{\vec{k}} \widetilde{\mathrm{PV}} \int_{a} \widetilde{\mathrm{PV}} \int_{a^{\prime}} \int \frac{d k^{0}}{2 \pi} \sigma(k, a) \Delta(a) \Delta(P-k-a) \Delta(k) \\
\quad \times i \mathcal{K}_{2 ; \text { off }}\left(a, P-k-a,-a^{\prime}\right) \Delta\left(a^{\prime}\right) \Delta\left(P-k-a^{\prime}\right) \sigma^{\dagger}\left(k, a^{\prime}\right) .
\end{aligned}
$$


(a)

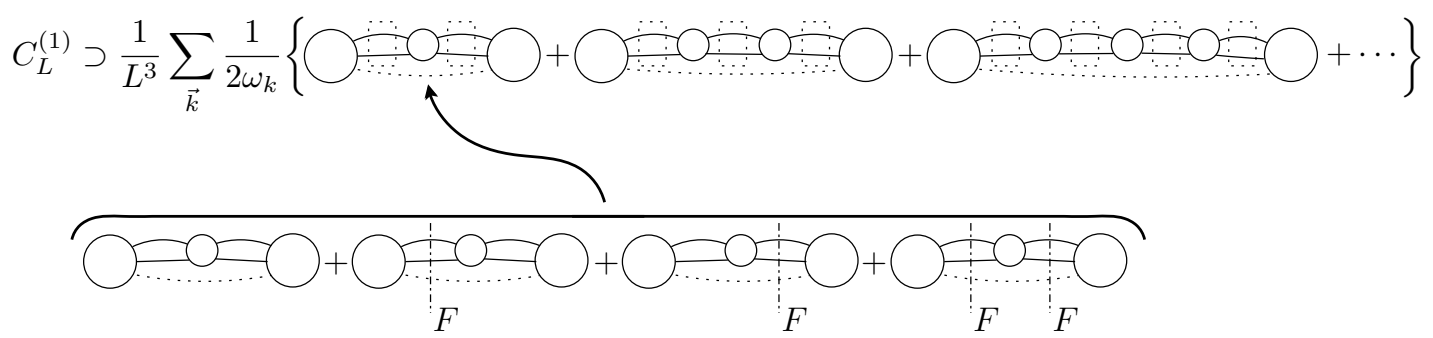

(b)

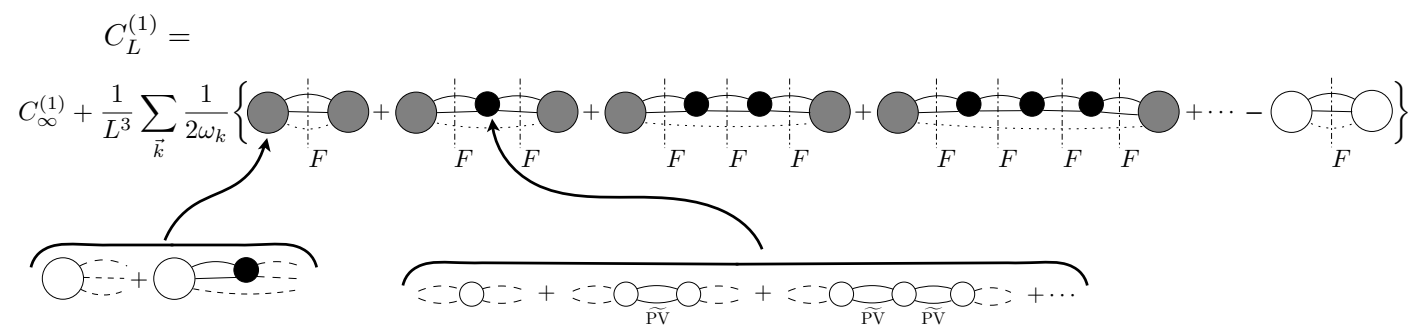

(c)

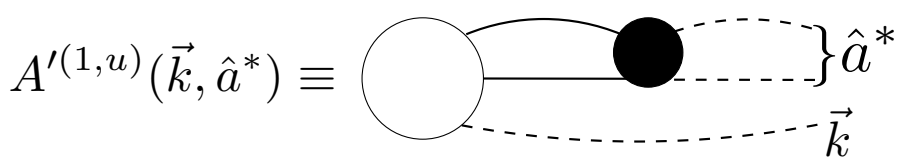

Figure 5.9: (a) Diagrams contributing power-law finite-volume contributions to $C_{L}^{(1)}$. The dashed line for the bottom propagator indicates that the $k^{0}$ integration has been done and only the one particle pole kept, giving rise to the factor of $1 / 2 \omega_{k}$. The inset shows the effect of substituting the identity of Fig. 5.8. (b) Result for $C_{L}^{(1)}$ after grouping terms according to the number of $F$ insertions. Diagrams with no insertions combine with the terms neglected in (a) to give $C_{\infty}^{(1)}$. In diagrams with at least one insertion of $F$ the factors to the left and right are $\sigma^{*}+A^{\prime(1, u)}$ and $\sigma^{\dagger *}+A^{(1, u)}$, respectively. The factors between $F$-insertions (denoted by black circles) are two-to-two K-matrices. The final term in the curly braces must be subtracted since it is included in the first term but is not part of the definition of $C_{L}^{(1)}$. (c) Definition of $A^{\prime(1, u)}$. The superscript $u$ indicates that the unscattered particle is also the particle whose momentum is singled out by the coordinate system. Dashed lines for external momenta indicate both that they are on shell and that they are amputated. 
Here we are using the definition of $\widetilde{P V}$ given in Eq. 5.66), while the off-shell K-matrix is

$$
\begin{array}{r}
i \mathcal{K}_{2 ; \text { off }}\left(a, b,-a^{\prime}\right)=i B_{2}\left(a, b,-a^{\prime}\right)+\frac{1}{2} \widetilde{\mathrm{PV}} \int_{a_{1}} i B_{2}\left(a, b,-a_{1}\right) \Delta\left(a_{1}\right) \Delta\left(b_{1}\right) i B_{2}\left(a_{1}, b_{1},-a^{\prime}\right) \\
+\left(\frac{1}{2}\right)^{2} \widetilde{\mathrm{PV}} \int_{a_{2}} \widetilde{\mathrm{PV}} \int_{a_{1}} i B_{2}\left(a, b,-a_{1}\right) \Delta\left(a_{1}\right) \Delta\left(b_{1}\right) i B_{2}\left(a_{1}, b_{1},-a_{2}\right) \Delta\left(a_{2}\right) \Delta\left(b_{2}\right) i B_{2}\left(a_{2}, b_{2},-a^{\prime}\right) \\
+\ldots, \quad(5.84)
\end{array}
$$

or equivalently

$$
\begin{aligned}
i \mathcal{K}_{2 ; \mathrm{off}}\left(a, b,-a^{\prime}\right)=i B_{2}(a, b & \left.-a^{\prime}\right) \\
& +\frac{1}{2} \widetilde{\mathrm{PV}} \int_{a_{1}} i B_{2}\left(a, b,-a_{1}\right) \Delta\left(a_{1}\right) \Delta\left(b_{1}\right) i \mathcal{K}_{2 ; \mathrm{off}}\left(a_{1}, b_{1},-a^{\prime}\right) .
\end{aligned}
$$

For both $\mathcal{K}_{2 \text {;off }}$ and $B_{2}$ we display only three of the (inflowing) momentum arguments, the fourth being given by momentum conservation: $a+b=a_{1}+b_{1}=a_{2}+b_{2}$. If all external momenta are on-shell, $\mathcal{K}_{2 \text {;off }}$ becomes the usual physical two-particle K-matrix $\mathcal{K}_{2}$, which is real and smooth (in our kinematic range) because the $\widetilde{\mathrm{PV}}$ prescription is identical to the $\mathrm{PV}$ prescription in this regime. Within $C_{\infty}^{(1)}$, the K-matrix is needed also below threshold, and our use of the $\widetilde{\mathrm{PV}}$ prescription ensures that $\mathcal{K}_{2 \text {;off }}$ is smooth (cusp-free) in this regime as well. These results allow the overall sum over $\vec{k}$ to be replaced with an integral (for which no pole prescription is needed).

We stress that in Eq. (5.83) the integral over $k^{0}$ must be done before the other loop integrals. This either puts the lower line on shell (leading to the cuts which are dealt with by the $\widetilde{\mathrm{PV}}$ prescription) or leads to intermediate states without a singularity (for which no pole prescription is needed). The need to keep track of the ordering of integrals is an unpleasant feature of the $\widetilde{\mathrm{PV}}$ prescription.

We next sum all terms with exactly one $F$ insertion, obtaining

$$
\begin{aligned}
C_{L, 1 F}^{(1)} & =\sigma^{*} \frac{i F}{2 \omega L^{3}} A^{(1, u)}+A^{\prime(1, u)} \frac{i F}{2 \omega L^{3}} \sigma^{\dagger *}+A^{\prime(1, u)} \frac{i F}{2 \omega L^{3}} A^{(1, u)} \\
& =\left(\sigma^{*}+A^{\prime(1, u)}\right) \frac{i F}{2 \omega L^{3}}\left(\sigma^{\dagger *}+A^{(1, u)}\right)-\sigma^{*} \frac{i F}{2 \omega L^{3}} \sigma^{\dagger *}
\end{aligned}
$$

Here $\sigma^{*}$ and $A^{\prime(1, u)}\left(\sigma^{\dagger *}\right.$ and $\left.A^{(1, u)}\right)$ are understood as row (column) vectors in the $k, \ell, m$ space introduced above. The vectors $\sigma^{*}$ and $\sigma^{\dagger *}$ have been defined in Eq. 5.74, while 
$A^{\prime(1, u)}$ and $A^{(1, u)}$ are new. To define these, we begin with the functions

$$
\begin{aligned}
A^{\prime(1, u)}(\vec{k}, a) & \equiv \frac{1}{2} \widetilde{\mathrm{PV}} \int_{a^{\prime}} \sigma\left(a^{\prime}, k\right) \Delta\left(a^{\prime}\right) \Delta\left(P-k-a^{\prime}\right) i \mathcal{K}_{2 ; \mathrm{off}}\left(a^{\prime}, P-k-a^{\prime},-a\right), \\
A^{(1, u)}(\vec{k}, a) & \equiv \frac{1}{2} \widetilde{\mathrm{PV}} \int_{a^{\prime}} i \mathcal{K}_{2 ; \mathrm{off}}\left(a, P-k-a,-a^{\prime}\right) \Delta\left(a^{\prime}\right) \Delta\left(P-k-a^{\prime}\right) \sigma^{\dagger}\left(a^{\prime}, k\right),
\end{aligned}
$$

in which $k=\left[\omega_{k}, \vec{k}\right]$ is on shell while $a$ is not. The superscripts $u$ indicate that the first momentum argument (here $\vec{k}$ ) is also the momentum of the particle that is unscattered by the two-to-two K-matrix. We next set the momenta $k$ and $a$ on shell, convert to $\mathrm{CM}$ coordinates for the scattered particles, and decompose in spherical harmonics ${ }^{24}$

$$
\begin{gathered}
A_{\ell^{\prime}, m^{\prime}}^{\prime(1, u)}\left(\vec{k}, a^{*}\right) \sqrt{4 \pi} Y_{\ell^{\prime}, m^{\prime}}\left(\hat{a}^{*}\right) \equiv A^{\prime(1, u)}\left(\vec{k},\left[\omega_{a}, \vec{a}\right]\right), \\
\sqrt{4 \pi} Y_{\ell, m}^{*}\left(\hat{a}^{*}\right) A_{\ell, m}^{(1, u)}\left(\vec{k}, a^{*}\right) \equiv A^{(1, u)}\left(\vec{k},\left[\omega_{a}, \vec{a}\right]\right) .
\end{gathered}
$$

Finally we project on-shell and restrict to finite-volume momenta

$$
A_{k, \ell^{\prime}, m^{\prime}}^{\prime(1, u)} \equiv A_{\ell^{\prime}, m^{\prime}}^{(1, u)}\left(\vec{k}, q_{k}^{*}\right) \text { and } A_{k, \ell, m}^{(1, u)} \equiv A_{\ell, m}^{(1, u)}\left(\vec{k}, q_{k}^{*}\right) \text {, with } \vec{k}, \vec{k}^{\prime} \in(2 \pi / L) \mathbb{Z}^{3} \text {. }
$$

This gives the vector forms appearing in Eq. 5.86). The diagrammatic definition of $A^{\prime(1, u)}$ is given in Fig. 5.9.

To see that Eq. (5.86) is valid, first observe that terms with a single $F$ insertion fall into three classes: (1) those with no $B_{2}$ kernels to the left of the $F$ insertion but one or more to the right; (2) those with no kernels to the right but one or more to the left; (3) those with one or more $B_{2}$ kernels on both sides of the single $F$ insertion. These give rise, respectively, to the three terms in Eq. (5.86), after performing the sums over insertions of $B_{2}$ to obtain the factors of $\mathcal{K}_{2 \text {;off }}$ contained in $A^{\prime(1, u)}$ and $A^{(1, u)}$. Finally, observe that coordinates that are commom with the single $F$-insertion are projected onto the on-shell, finite-volume phase space, leading to the now-familiar matrix structure.

At this stage we can easily generalize to terms with $(n>1) F$ insertions between $B_{2}$ kernels. We find

$$
C_{L, n F}^{(1)}=\left(\sigma^{*}+A^{\prime(1, u)}\right) \frac{i F}{2 \omega L^{3}}\left[i \mathcal{K}_{2} i F\right]^{n-1}\left(\sigma^{\dagger *}+A^{(1, u)}\right) .
$$

\footnotetext{
${ }^{24}$ Note that here we do not add a superscript ${ }^{*}$ to $A$ and $A^{\prime}$ when one of the momenta is in the CM frame. This would make the notation too heavy. The presence of the harmonic subscripts $\ell, m$ serves as an alternative indicator that we are using a CM momentum.
} 
Here we are using the matrix definition of $\mathcal{K}_{2}$ given in Eq. (5.11). In words, this says that, between insertions of $F$, one can have any number of $B_{2}$ 's connected by $\widetilde{P V}$ integrals, and these sum to give $\mathcal{K}_{2}$. Summing over $n$, including the $n=0$ result $C_{\infty}^{(1)}$, we obtain

$$
C_{L}^{(1)}=C_{\infty}^{(1)}+\left(\sigma^{*}+A^{\prime(1, u)}\right)[\mathcal{A}]\left(\sigma^{\dagger *}+A^{(1, u)}\right)-\sigma^{*} \frac{i F}{2 \omega L^{3}} \sigma^{\dagger *}
$$

where

$$
\mathcal{A} \equiv \frac{i F}{2 \omega L^{3}} \frac{1}{1+\mathcal{K}_{2} F}=\frac{1}{1+F \mathcal{K}_{2}} \frac{i F}{2 \omega L^{3}} .
$$

Combining with our earlier expression 5.75 for $C_{L}^{(0)}$ gives the main result of this subsection

$$
C_{L}^{(0)}+C_{L}^{(1)}=C_{\infty}^{(0)}+C_{\infty}^{(1)}+\left(\sigma^{*}+A^{\prime(1, u)}\right)[\mathcal{A}]\left(\sigma^{\dagger *}+A^{(1, u)}\right)-(2 / 3) \sigma^{*} \frac{i F}{2 \omega L^{3}} \sigma^{\dagger *} .
$$

We have succeeded in separating the correlator into factors of $F$, which depend on the volume, and infinite-volume quantities.

The calculation just described follows very closely the derivation of the two-particle quantization condition in a moving frame given in Ref. [130]. This is because, for the diagrams of Fig. 5.7, the third particle is a spectator whose main impact is to take momentum away from the other two particles. One difference in the present calculation, however, is that the $1 / 6$ symmetry factor for the no-insertion diagram, Fig. 5.6, is such that it does not match with those in the geometric sum leading to the factor of $[\mathcal{A}]$ in the second term in Eq. (5.96). This is the reason for the appearance of the last term in our result.

We can make the connection to the result of Ref. [130] more precise by considering instead the theory in which the spectator is of a different type from the other two particles and does not interact. For such a theory the symmetry factor for Fig. 5.6 is $1 / 2$, and the last term in Eq. (5.96) is absent. Indeed, for this theory we have already calculated all possible diagrams, with the final result

$$
C_{L}^{2+\text { spec }}-C_{\infty}^{2+\text { spec }}=\left(\sigma^{*}+A^{\prime(1, u)}\right)[\mathcal{A}]\left(\sigma^{\dagger *}+A^{(1, u)}\right)
$$

The spectrum is given by the poles of $C_{L}$. Since infinite-volume quantities do not lead to poles, $C_{L}$ diverges if and only if $[\mathcal{A}]$ has a divergent eigenvalue. This gives the quantization condition

$$
\operatorname{det}\left[\mathcal{K}_{2}^{-1}+F\right]=0
$$


where the determinant is over our [finite-volume momentum] $\times$ [angular momentum] space. Because both $i \mathcal{K}_{2 ; k^{\prime}, \ell^{\prime}, m^{\prime} ; k, \ell, m}$ and $i F_{k^{\prime}, \ell^{\prime}, m^{\prime} ; k, \ell, m}$ are diagonal in $k, k^{\prime}$ space, this condition may be rewritten as

$$
\prod_{\vec{k}} D(\vec{k})=0
$$

where

$$
D(\vec{k}) \equiv \underset{\text { ang mom }}{\operatorname{det}}\left[\mathcal{K}_{2}(\vec{k})^{-1}+F(\vec{k})\right]
$$

The quantities appearing in this equation are defined in Eqs. (5.11) and (5.74), and have only angular-momentum indices, since $\vec{k}$ is fixed.

This result is exactly what we expect given given the two-particle quantization condition of Ref. [130]. To see this, we note that, using Eqs. 55.59 and 5.60 to convert the PV into the $i \epsilon$ prescription, $\mathcal{M}_{2 ; \ell^{\prime}, m^{\prime} ; \ell, m}(\vec{k})$ is related to $\mathcal{K}_{2 ; \ell^{\prime}, m^{\prime} ; \ell, m}(\vec{k})$ by

$$
i \mathcal{M}_{2}=i \mathcal{K}_{2}+i \mathcal{K}_{2}(i \rho) i \mathcal{K}_{2}+\cdots=i \mathcal{K}_{2} \frac{1}{1+\rho \mathcal{K}_{2}}
$$

Here all arguments and indices are implicit. It follows that

$$
\mathcal{M}_{2}^{-1}(\vec{k})-\mathcal{K}_{2}^{-1}(\vec{k})=\rho(\vec{k})=F(\vec{k})-F^{i \epsilon}(\vec{k})
$$

where the last equality follows from Eq. (5.72). Thus we can rewrite the quantity appearing in the "2+spec" quantization condition as

$$
D(\vec{k}) \equiv \underset{\text { ang mom }}{\operatorname{det}}\left[\mathcal{M}_{2}(\vec{k})^{-1}+F^{i \epsilon}(\vec{k})\right]
$$

If this vanishes for one of the finite-volume choices of $\vec{k}$, then there is a finite-volume state in the "2+spec" theory.

The connection to the result of Ref. [130] can now be made. If the spectator, which is necessarily on-shell since it is non-interacting, has momentum $\left[\omega_{k}, \vec{k}\right]$, then the total momentum of the other two particles is $P_{2}=\left[E-\omega_{k}, \vec{P}-\vec{k}\right]$. For the full "2+spec" theory to have a finite-volume state, the two interacting particles with momentum $P_{2}$ must have a finite-volume state. The condition for this, as given in Ref. [130], is exactly $D(\vec{k})=0$. This agreement provides a useful check on our formalism 25

\footnotetext{
${ }^{25}$ Note that $F^{i \epsilon}$ (and not $F$ ) is the kinematic factor derived in Ref. [130. Also, the ultra-violet cut-off
} 


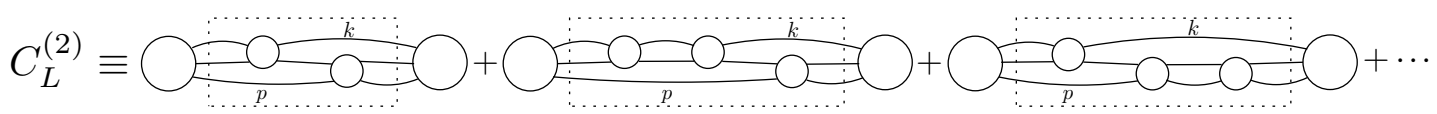

Figure 5.10: Subset of finite-volume correlator diagrams containing only two-to-two insertions, with one switch in the scattered pair.

\subsubsection{Two-to-two insertions: one switch}

In this section we sum the diagrams of Fig. 5.10. Each diagram has at least one $B_{2}$ insertion on exactly two different pairs of particles. In other words, the diagrams have one switch in the pair that is scattered. We denote the sum of all such diagrams by $C_{L}^{(2)}$. Throughout this section we call the momentum of the incoming spectator particle $k$ and that of the outgoing spectator $p$, as shown in the figure. We refer to the three propagators which appear at the location where the scattered pair changes as the "switch state". The presence of a switch leads to the first appearance of a three-particle scattering quantity in our analysis.

To determine the volume-dependent contribution of these diagrams we first evaluate the $p^{0}$ and $k^{0}$ integrals. Since we know from earlier considerations that intermediate states with

used in the definition of $F^{i \epsilon}$ in Ref. [130] differs from that we use, but this leads only to a difference that is exponentially suppressed in $L$. Finally, we take this opportunity to comment on potential confusion regarding the definitions in earlier papers of $F^{i \epsilon}$ below two-particle threshold. In particular in Ref. [140], for example in Eqs. (24) and (25), the above-threshold definition of $F^{i \epsilon}$ is split into real and imaginary parts, with the principal-value pole prescription used to define the latter. In contrast to the $\widetilde{P V}$ prescription of the present article, the principal-value in [140] is replaced with a simple prescription-free integral below threshold. In addition, the imaginary part of $F^{i \epsilon}$, the term that we call $\rho$ here, is set to zero below threshold in Ref. [140]. The upshot is that the difference between $\widetilde{P V}$ used here and principal-value in [140] exactly cancels the difference between $\rho$ defined here and the analog in [140, so that the definition of $F^{i \epsilon}$ is consistent in the two papers. A useful pneumonic to keep track of this issue generally is as follows: When the quantization condition is written in terms of $\mathcal{M}_{2}^{-1}$, then the finite-volume quantity added to the inverted scatteringamplitude should become exponentially suppressed below two-particle threshold. This ensures that one recovers the correct finite-volume bound state condition. 
three on-shell particles are needed to obtain power-law volume dependence, at least one of the two poles at $p^{0}=\omega_{p}$ and $k^{0}=\omega_{k}$ must be encircled. For concreteness we enumerate the four types of terms: (a) each contour encircles its one-particle pole; (b) the $p^{0}$ contour encircles its pole but the $k^{0}$ contour encircles all other contributions; (c) as in (b) but with $k^{0}$ and $p^{0}$ exchanged; and (d) both contours encircle everything but the one-particle poles. We now consider the loop sums/integrals that remain when holding $\vec{p}$ and $\vec{k}$ fixed; these are all two-particle loops involving either the upper two particles (to the left of the switch state) or the lower two (to the right). For type (d) terms the summands have no singularities and thus all sums can be replaced with integrals. Similarly, in type (b) and (c) terms, the twoparticle loops on one side of the switch state cannot go on-shell and may thus be replaced by integrals. For all remaining two-particle loops in terms of types (a), (b) and (c), the summand is singular. Here we substitute the identity of Eq. (5.75), thereby separating each loop into an infinite-volume contribution and an $F$-factor residue.

There are thus two disjoint regions where insertions of $F$ appear: to the left of the switch state and to the right. It is useful to break our analysis into four classes, defined by whether or not each side of the switch state has at least one insertion. We label these as

$$
\text { (1) } F, F, \quad(2)-, F, \quad(3) F,-, \quad(4)-,-,
$$

so that class (1) contains all terms with at least one $F$ insertion both to the left and right of the switch state, class (2) contains terms with no such insertions to the left but at least one to the right, etc. Observe that type (a) terms appear in all four classes, while types (b) and $(\mathrm{c})$ only appear in classes $(2+4)$ and $(3+4)$ respectively.

We now analyze the four classes in turn, starting with (1). Because all terms in this class have both $k^{0}$ and $p^{0}$ one-particle poles, the chains of $F$ 's, $B_{2}$ 's and $\widetilde{P V}$-integrated loops to the left and right of the switch state can each be independently summed exactly as in the previous subsection. This leads to

$$
\left(\sigma^{*}+A^{\prime(1, u)}\right)[\mathcal{A}] i \mathcal{K}_{3}^{(2, u, u)}[\mathcal{A}]\left(\sigma^{\dagger *}+A^{(1, u)}\right) .
$$

The new feature here is the quantity $\mathcal{K}_{3}^{(2, u, u)} \equiv \mathcal{K}_{3 ; p, \ell, m ; k, \ell^{\prime}, m^{\prime}}^{(2, u, u)}$ which arises from the switch state, and is a contribution to the three-to-three scattering amplitude. It is shown diagram- 
matically in Fig. 5.11a, to which we refer for the notation for momenta. To define it we proceed in the by-now familiar steps, beginning with the partially off-shell quantity

$$
i \mathcal{K}_{3}^{(2, u, u)}\left(\vec{p}, \vec{a}, \vec{k}, \vec{a}^{\prime}\right) \equiv i \mathcal{K}_{2 ; \mathrm{off}}(a, P-p-a,-k) \Delta(P-p-k) i \mathcal{K}_{2 ; \mathrm{off}}\left(P-p-k, p,-a^{\prime}\right) .
$$

At this stage $p, k, a$ and $a^{\prime}$ are on shell, while $P-p-k, P-p-a$ and $P-k-a^{\prime}$ are not. We have parametrized $i \mathcal{K}_{3}^{(2, u, u)}$ with incoming and outgoing spectator momenta, $\vec{k}$ and $\vec{p}$, as well as incoming and outgoing momenta of one of the scatterers, $\vec{a}^{\prime}$ and $\vec{a}$. In the second step we change the frame used to define the scatterer momenta and then we decompose in spherical harmonics

$$
\mathcal{K}_{3}^{(2, u, u)}\left(\vec{p}, \vec{a}, \vec{k}, \vec{a}^{\prime}\right) \equiv 4 \pi Y_{\ell, m}^{*}\left(\hat{a}^{*}\right) \mathcal{K}_{3 ; \ell, m ; \ell^{\prime}, m^{\prime}}^{(2, u, u)}\left(\vec{p}, a^{*}, \vec{k}, a^{\prime *}\right) Y_{\ell, m}\left(\hat{a}^{\prime *}\right)
$$

where $\vec{a}^{*}$ is defined by boosting $\left(\omega_{a}, \vec{a}\right) \rightarrow\left(\omega_{a}^{*}, \vec{a}^{*}\right)$ with velocity $\vec{\beta}_{p}$, and $\vec{a}^{\prime *}$ is defined by boosting the corresponding primed vector with $\vec{\beta}_{k}$. Next we recall that all incoming and outgoing particles are on-shell if and only if $a^{\prime *}=q_{k}^{*}$ and $a^{*}=q_{p}^{*}$. Thus we define the on-shell version of $\mathcal{K}_{3}^{(2, u, u)}$ as

$$
\mathcal{K}_{3 ; \ell, m ; \ell^{\prime}, m^{\prime}}^{(2, u, u)}(\vec{p}, \vec{k}) \equiv \mathcal{K}_{3 ; \ell, m ; \ell^{\prime}, m^{\prime}}^{(2, u, u)}\left(\vec{p}, q_{p}^{*}, \vec{k}, q_{k}^{*}\right)
$$

The final step is to restrict to finite-volume momenta

$$
\mathcal{K}_{3 ; p, \ell, m ; k, \ell^{\prime}, m^{\prime}}^{(2, u, u)} \equiv \mathcal{K}_{3 ; \ell, m ; \ell^{\prime}, m^{\prime}}^{(2, u, u)}(\vec{p}, \vec{k}) \quad \text { for } \quad \vec{k}, \vec{p} \in(2 \pi / L) \mathbb{Z}^{3}
$$

This gives the matrix contained in the result Eq. (5.105).

Several further explanations are in order. First, $\mathcal{K}_{3}^{(2, u, u)}$ in Eq. 5.105 is on-shell on both "sides" because it is sandwiched between factors of $F$. This is because $[\mathcal{A}]$, defined in Eq. (5.95), has an $F$ on both ends. Second, the boosts to CM momenta $\vec{a}^{*}$ and $\vec{a}^{*}$ are always well defined because $F$ contains factors of $H(\vec{p})$ (on the left) and $H(\vec{k})$ (on the right). Third, sub-threshold momenta occur in both left and right CM frames as $\vec{p}$ and $\vec{k}$ are varied, requiring analytic continuation of the $\mathcal{K}_{3}^{(2, u, u)}$. Fourth, all factors from external propagators are contained in the $[\mathcal{A}]^{\prime}$ 's, so $\mathcal{K}_{3}^{(2, u, u)}$ is a contribution to the amputated three-tothree scattering amplitude. Fifth, the superscript " $(2, u, u)$ " indicates that this contribution involves two factors of $\mathcal{K}_{2 \text {;off }}$, and that, on both sides, the particles singled out by the label 
(a)

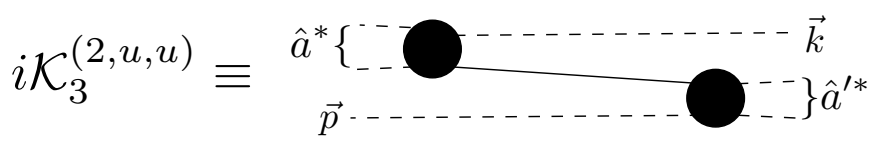

(b)

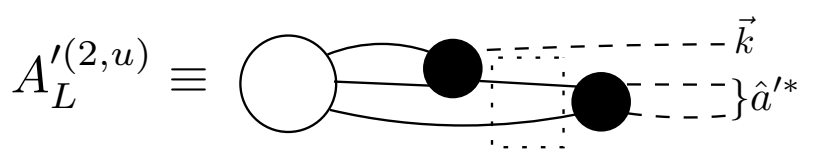

$(c)$

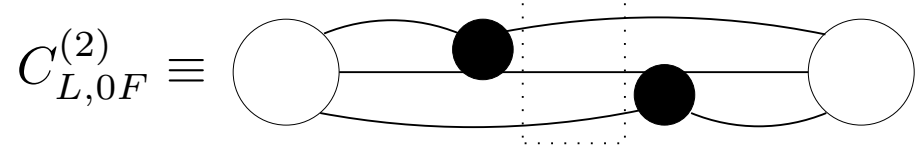

Figure 5.11: Diagrammatic definitions of (a) $i \mathcal{K}_{3}^{(2, u, u)}$, (b) $A_{L}^{\prime(2, u)}$ and (c) $C_{L, 0 F}^{(2)}$. In (b) and (c) the dotted box encloses momenta that are summed rather than integrated. The solid circle represents the two-particle K-matrix. Other notation as above.

( $\vec{p}$ on the left, $\vec{k}$ on the right) are unscattered. And, finally, although the result 5.105 has a symmetric form, it is important to note that $\mathcal{K}_{3}^{(2, u, u)}$ switches the spectator momentum index from $p$ to $k$.

We now turn our attention to class (2) contributions, i.e. those with no $F$ insertions to the left of the switch state but at least one such insertion on the right. As noted above, these contributions come from terms of types (a) and (b). In the former, the $p^{0}$ and $k^{0}$ integrals both encircle one-particle poles, but all two-particle loops with $p$ as the spectator are integrated using the $\widetilde{\mathrm{PV}}$ prescription. In the latter, only the $k^{0}$ integral encircles the one-particle pole, so all two-particle loop sums to the left of the switch state can be replaced by integrals. Combining these contributions, we find

$$
A_{L}^{\prime(2, u)}[\mathcal{A}]\left(\sigma^{\dagger *}+A^{(1, u)}\right)
$$


where the new quantity $A_{L}^{(2, u)}$ is shown diagrammatically in Fig. 5.11b. It is a contribution to the left end-cap involving one switch. It is given by

$$
A_{L ; k, \ell^{\prime}, m^{\prime}}^{(2, u)} \equiv A_{L ; \ell^{\prime}, m^{\prime}}^{(2, u)}\left(\vec{k}, q_{k}^{*}\right) \quad\left[\text { with } k \in(2 \pi / L) \mathbb{Z}^{3}\right]
$$

where

$$
A_{L ; \ell^{\prime}, m^{\prime}}^{\prime(2, u)}\left(\vec{k}, a^{\prime *}\right) \sqrt{4 \pi} Y_{\ell^{\prime}, m^{\prime}}\left(\hat{a}^{\prime *}\right) \equiv A_{L}^{\prime(2, u)}\left(\vec{k},\left[\omega_{a^{\prime}}, \vec{a}^{\prime}\right]\right)
$$

and

$$
\begin{aligned}
A_{L}^{\prime(2, u)}\left(\vec{k}, a^{\prime}\right) \equiv & \frac{1}{2} \frac{1}{L^{3}} \sum_{\vec{p}} \widetilde{\mathrm{PV}} \int_{a} \int_{p^{0}} \sigma(p, a) \Delta(a) \Delta(P-p-a) \\
& \times i \mathcal{K}_{2 ; \mathrm{off}}(a, P-p-a,-k) \Delta(p) \Delta(P-p-k) i \mathcal{K}_{2 ; \mathrm{off}}\left(p, P-p-k,-a^{\prime}\right)
\end{aligned}
$$

[with $\left.\int_{p^{0}} \equiv \int d p^{0} /(2 \pi)\right]$ is the end-cap amplitude with $k$ on shell but $a$ not. The subscript $L$ is a reminder that this quantity contains important finite-volume effects. These arise from the sum over $\vec{p}$ with a singular summand (from the switch state). The superscript $(2, u)$ refers to the presence of two factors of $\mathcal{K}_{2 \text {;off }}$ and the fact that the particle carrying the momentum that is singled out by the coordinate system (here $\vec{k}$ ) is unscattered.

Class (3) contributions mirror those from class (2), with the roles of the parts of the diagrams to the left and right of the switch state interchanged. The total result is

$$
\left(\sigma^{*}+A^{\prime(1, u)}\right)[\mathcal{A}] A_{L}^{(2, u)},
$$

where

$$
A_{L ; p, \ell, m}^{(2, u)} \equiv A_{L ; \ell, m}^{(2, u)}\left(\vec{p}, q_{p}^{*}\right) \quad\left[\text { with } p \in(2 \pi / L) \mathbb{Z}^{3}\right]
$$

with

$$
A_{L ; \ell, m}^{(2, u)}\left(\vec{p}, a^{*}\right) \sqrt{4 \pi} Y_{\ell^{\prime}, m^{\prime}}\left(\hat{a}^{*}\right) \equiv A_{L}^{(2, u)}\left(\vec{p},\left[\omega_{a}, \vec{a}\right]\right)
$$

and

$$
\begin{aligned}
A_{L}^{(2, u)}(\vec{p}, a) \equiv \frac{1}{2} \frac{1}{L^{3}} \sum_{\vec{k}} \widetilde{\mathrm{PV}} \int_{a^{\prime}} \int_{k^{0}} i \mathcal{K}_{2 ; \mathrm{off}}(a, P-p-a,-k) \Delta(k) \Delta(P-p-k) \\
\quad \times i \mathcal{K}_{2 ; \mathrm{off}}\left(p, P-p-k,-a^{\prime}\right) \Delta\left(a^{\prime}\right) \Delta\left(P-k-a^{\prime}\right) \sigma^{\dagger}\left(k, a^{\prime}\right) .
\end{aligned}
$$


Finally, we turn to class (4) contributions, which have no $F$ insertions on either side of the switch state. Combining contributions from types (a-d), we find [see Fig. 5.11k]

$$
\begin{aligned}
C_{L, 0 F}^{(2)}=\frac{1}{4} & \frac{1}{L^{6}} \sum_{\vec{p}, \vec{k}} \widetilde{\mathrm{PV}} \int_{a, a^{\prime}} \int_{p^{0}} \int_{k^{0}} \sigma(p, a) \Delta(a) \Delta(P-p-a) \Delta(p) i \mathcal{K}_{2 ; \mathrm{off}}(a, P-p-a,-k) \\
& \times \Delta(P-p-k) i \mathcal{K}_{2 ; \mathrm{off}}\left(P-p-k, p,-a^{\prime}\right) \Delta\left(a^{\prime}\right) \Delta\left(P-k-a^{\prime}\right) \Delta(k) \sigma^{\dagger}\left(k, a^{\prime}\right) .
\end{aligned}
$$

Adding this to the results from the other classes, we obtain

$$
\begin{aligned}
C_{L}^{(2)}=\left(\sigma^{*}+A^{\prime(1, u)}\right)[\mathcal{A}] i \mathcal{K}_{3}^{(2, u, u)}[\mathcal{A}]\left(\sigma^{\dagger *}+A^{(1, u)}\right) & +A_{L}^{(2, u)}[\mathcal{A}]\left(\sigma^{\dagger *}+A^{(1, u)}\right) \\
& +\left(\sigma^{*}+A^{\prime(1, u)}\right)[\mathcal{A}] A_{L}^{(2, u)}+C_{L, 0 F}^{(2)}
\end{aligned}
$$

At this stage we have achieved only a partial separation of finite-volume effects, because $A_{L}^{(2, u)}, A_{L}^{(2, u)}$ and $C_{L, 0 F}^{(2)}$ still contain momentum sums that cannot be replaced by integrals. In addition, $\mathcal{K}_{3}^{(2, u, u)}$ suffers from the problem, discussed in the introduction, of diverging for certain physical momenta. In the remainder of this section we derive identities for these four quantities that allow a complete separation of finite-volume effects and avoid divergences in the $3 \rightarrow 3$ scattering amplitude.

We begin with $\mathcal{K}_{3}^{(2, u, u)}$, and separate it into two terms, one which is singular but only depends on the on-shell $\mathcal{K}_{2}$, and another which is regular. We do this separation in a way that allows generalization to diagrams with more switches. In particular, we will analyze the partially off-shell quantity $\mathcal{K}_{3}^{(2, u, u)}\left(\vec{p}, \vec{a}, \vec{k}, \vec{a}^{\prime}\right)$, defined in Eq. 5.106), although for this subsection we only need the on-shell version of this quantity [as in Eq. (5.108)]. In fact, we keep the four momentum arguments completely general so that the boosts to the CM-frames for $a$ and $a^{\prime}$ need not be defined.

Our first step is to write the intermediate propagator as

$$
\Delta(P-p-k)=\frac{i H(\vec{p}) H(\vec{k})}{2 \omega_{k p}\left(E-\omega_{p}-\omega_{k}-\omega_{k p}\right)}+\mathcal{R}^{a}(\vec{p}, \vec{k}) .
$$

The first term contains the on-shell singularity, while the second is smooth. We focus for now on the singular term in 5.120 and substitute this into $\mathcal{K}_{3}^{(2, u, u)}$, Eq. 5.106). The presence of the $H$ factors means that we can boost to the CM frames for the $\{k, P-p-k\}$ 
and the $\{P-p-k, p\}$ pairs, and decompose the dependence on $\vec{k}^{*}$ and $\vec{p}^{*}$ into spherical harmonics. The result is that the singular contribution becomes

$$
i \mathcal{K}_{3}^{(2, u, u)}\left(\vec{p}, \vec{a}, \vec{k}, \vec{a}^{\prime}\right) \supset i \mathcal{K}_{2 \| \text { off } \| \text { off } \ell m}\left(\vec{p} ; \vec{a} \| k^{*}\right) i G_{\ell, m ; \ell^{\prime}, m^{\prime}}^{a}(\vec{p}, \vec{k}) i \mathcal{K}_{2 \| \text { off } \ell^{\prime} m^{\prime} \| \text { off }}\left(\vec{k} ; p^{*} \| \vec{a}^{\prime}\right)
$$

where

$$
i G_{\ell, m ; \ell^{\prime}, m^{\prime}}^{a}(\vec{p}, \vec{k}) \equiv \frac{i 4 \pi Y_{\ell, m}\left(\hat{k}^{*}\right) H(\vec{p}) H(\vec{k}) Y_{\ell^{\prime}, m^{\prime}}^{*}\left(\hat{p}^{*}\right)}{2 \omega_{p k}\left(E-\omega_{p}-\omega_{k}-\omega_{p k}\right)}
$$

and

$$
\begin{gathered}
\sqrt{4 \pi} \mathcal{K}_{2 \| \text { off } \| \text { off } \ell m}\left(\vec{p} ; \vec{a} \| k^{*}\right) Y_{\ell, m}\left(\hat{k}^{*}\right) \equiv \mathcal{K}_{2 ; \text { off }}(a, P-p-a,-k) \\
\sqrt{4 \pi} Y_{\ell^{\prime}, m^{\prime}}^{*}\left(\hat{p}^{*}\right) \mathcal{K}_{2 \| \text { off } \ell^{\prime} m^{\prime} \| \text { off }}\left(\vec{k} ; p^{*} \| \vec{a}^{\prime}\right) \equiv \mathcal{K}_{2 ; \text { off }}\left(P-p-k, p,-a^{\prime}\right) .
\end{gathered}
$$

In the latter two definitions, the two subscripts "off" are a reminder that both incoming and outgoing scattering pairs, have one particle off-shell. If the "off" is followed by angular momentum indices, this indicates that the scattered pair has been boosted to its CM frame and the angular dependence decomposed into spherical harmonics. The arguments

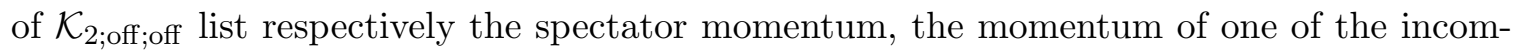
ing scattered pair, and the momentum of one of the outgoing pair. If a CM-frame boost has been done, the argument is the magnitude of the CM-frame momentum. This hybrid notation is needed to maintain generality.

The next step is to write the singular part of (5.121) in terms of on-shell K-matrices. This is straightforward as we can expand the boosted momenta $k^{*}$ and $p^{*}$ about their on-shell values, $q_{p}^{*}$ and $q_{k}^{*}$ respectively. At the same time, we want the remaining non-singular term to be a smooth function of $\vec{p}$ and $\vec{k}$, since this is required below. The spherical harmonics $Y_{\ell, m}\left(\hat{k}^{*}\right)$ and $Y_{\ell^{\prime}, m^{\prime}}^{*}\left(\hat{p}^{*}\right)$ are not, however, smooth at $\vec{k}^{*}=0$ (for $\ell>0$ ) and $\vec{p}^{*}=0$ (for $\ell^{\prime}>0$ ), respectively. To resolve this problem, and pull out an appropriate singular term, we introduce the finite difference operator $\delta$. This can act to the right or left on $\mathcal{K}_{2}$, with its action being

$$
\begin{aligned}
\delta \mathcal{K}_{2 \| \text { on } \ell^{\prime} m^{\prime} \| \text { off }}\left(\vec{k} ; \vec{a}^{\prime}\right) & \equiv \mathcal{K}_{2 \| \text { off } \ell^{\prime} m^{\prime} \| \text { off }}\left(\vec{k} ; p^{*} \| \vec{a}^{\prime}\right)-\left(p^{*} / q_{k}^{*}\right)^{\ell^{\prime}} \mathcal{K}_{2 \| \text { on } \ell^{\prime} m^{\prime} \| \text { off }}\left(\vec{k} ; \vec{a}^{\prime}\right) \\
\mathcal{K}_{2 \| \text { off } \| \text { on } \ell m}(\vec{p} ; \vec{a}) \delta & \equiv \mathcal{K}_{2 \| \text { off } \| \text { off } \ell m}\left(\vec{p} ; \vec{a} \| k^{*}\right)-\mathcal{K}_{2 \| \text { off } \| \text { on } \ell m}(\vec{p} ; \vec{a})\left(k^{*} / q_{p}^{*}\right)^{\ell}
\end{aligned}
$$


Here we have defined the "on-off" and "off-on" K-matrices as

$$
\begin{aligned}
\mathcal{K}_{2 \| \text { on } \ell^{\prime} m^{\prime} \| \text { off }}\left(\vec{k} ; \vec{a}^{\prime}\right) \equiv \mathcal{K}_{2 \| \text { off } \ell^{\prime} m^{\prime} \| \text { off }}\left(\vec{k} ; q_{k}^{*} \| \vec{a}^{\prime}\right) \\
\text { and } \\
\mathcal{K}_{2 \| \text { off } \| \text { on } \ell m}(\vec{p} ; \vec{a}) \equiv \mathcal{K}_{2 \| \text { off } \| \text { off } \ell m}\left(\vec{p} ; \vec{a} \| q_{p}^{*}\right)
\end{aligned}
$$

Note that if a scattering pair is on shell then it does not have a corresponding momentum argument (since the latter is fixed by kinematics).

Inserting Eqs. (5.125) and (5.126) into Eq. (5.121) we obtain

$$
\begin{aligned}
i \mathcal{K}_{3}^{(2, u, u)}\left(\vec{p}, \vec{a}, \vec{k}, \vec{a}^{\prime}\right) \supset \\
\quad i \mathcal{K}_{2 \| \text { off } \| \text { on } \ell m}(\vec{p} ; \vec{a})\left[i G_{\ell, m ; \ell^{\prime}, m^{\prime}}^{b}(\vec{p}, \vec{k})+\mathcal{R}_{\ell, m ; \ell^{\prime}, m^{\prime}}^{b}(\vec{p}, \vec{k})\right] i \mathcal{K}_{2 \| \text { on } \ell^{\prime} m^{\prime} \| \text { off }}\left(\vec{k} ; \vec{a}^{\prime}\right),
\end{aligned}
$$

with

$$
i G_{\ell, m ; \ell^{\prime}, m^{\prime}}^{b}(\vec{p}, \vec{k}) \equiv\left(\frac{k^{*}}{q_{p}^{*}}\right)^{\ell} \frac{i 4 \pi Y_{\ell, m}\left(\hat{k}^{*}\right) H(\vec{p}) H(\vec{k}) Y_{\ell^{\prime}, m^{\prime}}^{*}\left(\hat{p}^{*}\right)}{2 \omega_{p k}\left(E-\omega_{p}-\omega_{k}-\omega_{p k}\right)}\left(\frac{p^{*}}{q_{k}^{*}}\right)^{\ell^{\prime}}
$$

and

$$
\begin{aligned}
\mathcal{R}_{\ell, m ; \ell^{\prime}, m^{\prime}}^{b}(\vec{p}, \vec{k}) \equiv \delta i G_{\ell, m ; \ell^{\prime}, m^{\prime}}^{a} & (\vec{p}, \vec{k})\left(p^{*} / q_{k}^{*}\right)^{\ell^{\prime}} \\
& +\left(k^{*} / q_{p}^{*}\right)^{\ell} i G_{\ell, m ; \ell^{\prime}, m^{\prime}}^{a}(\vec{p}, \vec{k}) \delta+\delta i G_{\ell, m ; \ell^{\prime}, m^{\prime}}^{a}(\vec{p}, \vec{k}) \delta .
\end{aligned}
$$

The result 5.128 has achieved our goals. Only the $G^{b}$ term is singular, because the factors of $\delta$ in $\mathcal{R}^{b}$ [which act on the K-matrices appearing in Eq. [5.128] ] give differences which vanish when $P-p-k$ goes on shell and thus cancel the singularity in $G^{a}$. More precisely the analyticity of $\mathcal{K}_{2}$ near the on-shell point is required to demonstrate the cancellation. For example, the difference defined in Eq. (5.125) scales as $p^{*}-q_{k}^{*}$, the same scaling as the denominator of $G^{b}$, so that the product is a finite smooth function. This is discussed in detail in Appendix D. Furthermore, the extra powers of $k^{*}$ and $p^{*}$ ensure that $G^{b}$ is smooth when $\vec{k}^{*}$ or $\vec{p}^{*}$ vanish. Finally, the $G^{b}$ term multiplies K-matrices in which $k^{*}$ (to the left) and $p^{*}$ (to the right) are on shell.

The end result of this analysis is that

$$
\mathcal{K}_{3}^{(2, u, u)}\left(\vec{p}, \vec{a}, \vec{k}, \vec{a}^{\prime}\right)=\mathcal{D}^{(2, u, u)}\left(\vec{p}, \vec{a}, \vec{k}, \vec{a}^{\prime}\right)+\mathcal{K}_{\mathrm{df}, 3}^{(2, u, u)}\left(\vec{p}, \vec{a}, \vec{k}, \vec{a}^{\prime}\right)
$$



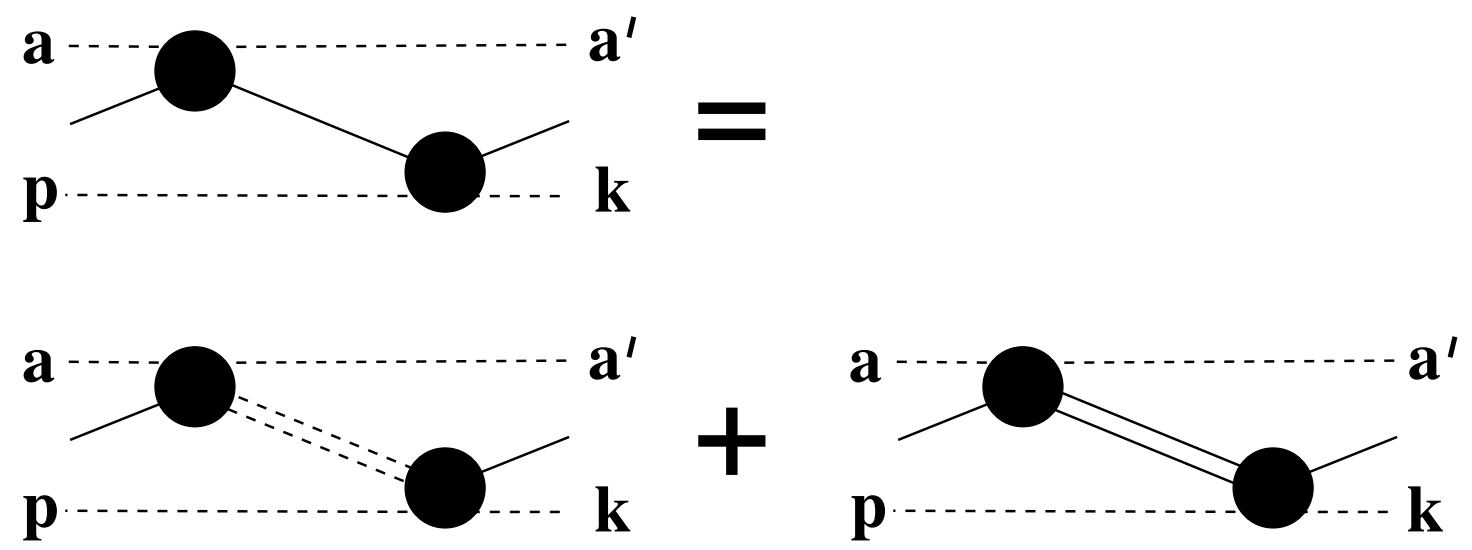

Figure 5.12: Diagrammatic version of the decomposition of $\mathcal{K}_{3}^{(2, u, u)}\left(\vec{p}, \vec{a}, \vec{k}, \vec{a}^{\prime}\right)$ given in Eq. (5.131). External dashed lines indicate on-shell momenta, whereas momenta flowing along the solid external lines are, in general, off shell. All external propagators are amputated. The first term on the right-hand side is the singular term $\mathcal{D}^{(2, u, u)}$, with the double dashed lines representing $G^{b}$. The two $\mathcal{K}_{2}$ are evaluated on-shell for all momenta that flow along dashed propagators. The second term represents the divergence-free amplitude $\mathcal{K}_{\mathrm{df}, 3}^{(2, u, u)}$.

where the singular part is

$$
i \mathcal{D}^{(2, u, u)}\left(\vec{p}, \vec{a}, \vec{k}, \vec{a}^{\prime}\right)=i \mathcal{K}_{2 \| \text { off } \| \text { on } \ell m}(\vec{p} ; \vec{a}) i G_{\ell, m ; \ell^{\prime}, m^{\prime}}^{b}(\vec{p}, \vec{k}) i \mathcal{K}_{2 \| \text { on } \ell^{\prime} m^{\prime} \| \text { off }}\left(\vec{k}, \vec{a}^{\prime}\right)
$$

and the divergence-free part of the amplitude is

$$
\begin{aligned}
i \mathcal{K}_{\mathrm{df}, 3}^{(2, u, u)}\left(\vec{p}, \vec{a}, \vec{k}, \vec{a}^{\prime}\right) \equiv i \mathcal{K}_{2 ; \mathrm{off}}(a, P-p-a,-k) \mathcal{R}^{a}(\vec{p}, \vec{k}) i \mathcal{K}_{2 ; \mathrm{off}}\left(P-p-k, p,-a^{\prime}\right) \\
+i \mathcal{K}_{2\|\mathrm{off}\| \mathrm{on} \ell m}(\vec{p}, \vec{a}) \mathcal{R}_{\ell, m ; \ell^{\prime}, m^{\prime}}^{b}(\vec{p}, \vec{k}) i \mathcal{K}_{2\left\|\mathrm{on} \ell^{\prime} m^{\prime}\right\| \mathrm{off}}\left(\vec{k}, \vec{a}^{\prime}\right) .
\end{aligned}
$$

The relation 5.131 is shown diagrammatically in Fig. 5.12 . The key property of $\mathcal{K}_{\mathrm{df}, 3}^{(2, u, u)}$ is that it is a smooth, non-singular function of its arguments. It is smooth when $\vec{k}^{*}$ or $\vec{p}^{*}$ vanish because $\mathcal{K}_{3}^{(2, u, u)}$ is smooth at these values and, as just discussed, this is also true of the $G^{b}$ term. 
The quantity $G^{b}$ is closely related to the matrix $G$ introduced in Eq. 5.21. In particular,

$$
G_{\ell, m ; \ell^{\prime}, m^{\prime}}^{b}(\vec{p}, \vec{k})=G_{p, \ell, m ; k_{1}, \ell_{1}, m_{1}}\left[2 \omega L^{3}\right]_{k_{1}, \ell_{1}, m_{1} ; k, \ell^{\prime}, m^{\prime}} \text { for } p, k \in(2 \pi / L) \mathbb{Z}^{3}
$$

where

$$
\left[2 \omega L^{3}\right]_{k_{1}, \ell_{1}, m_{1} ; k, \ell^{\prime}, m^{\prime}} \equiv \delta_{k_{1}, k} \delta_{\ell_{1}, \ell^{\prime}} \delta_{m_{1}, m^{\prime}} 2 \omega_{k} L^{3}
$$

Finally, with this groundwork laid, we can return to the quantity relevant for the oneswitch analysis, namely $\mathcal{K}_{3}^{(2, u, u)}$ with external momenta on shell and taking finite-volume values. In this case we can decompose the external CM-frame momenta in spherical harmonics, and connect back to our matrix notation:

$$
\begin{aligned}
\left.\mathcal{K}_{2 \| \text { off } \| \text { on } \ell m}(\vec{p} ; \vec{a})\right|_{a^{*}=q_{p}^{*}, \vec{p} \in(2 \pi / L) \mathbb{Z}^{3}} & =\sqrt{4 \pi} Y_{\ell^{\prime}, m^{\prime}}^{*}\left(\hat{a}^{*}\right) \mathcal{K}_{2 ; p, \ell^{\prime}, m^{\prime} ; p, \ell, m} \\
\left.\mathcal{K}_{2 \| \text { on } \ell m \| \text { off }}\left(\vec{k} ; \vec{a}^{\prime}\right)\right|_{a^{\prime *}=q_{k}^{*}, \vec{k} \in(2 \pi / L) \mathbb{Z}^{3}} & =\mathcal{K}_{2 ; k, \ell, m ; k, \ell^{\prime}, m^{\prime}} \sqrt{4 \pi} Y_{\ell^{\prime}, m^{\prime}}\left(\hat{a}^{\prime *}\right) .
\end{aligned}
$$

This allows us to write the decomposition into singular and smooth parts in matrix form

$$
i \mathcal{K}_{3 ; p, \ell, m ; k, \ell^{\prime}, m^{\prime}}^{(2, u, u)} \equiv i \mathcal{K}_{2} i G\left[2 \omega L^{3}\right] i \mathcal{K}_{2}+i \mathcal{K}_{\mathrm{df}, 3 ; p, \ell, m ; k, \ell^{\prime}, m^{\prime}}^{(2, u, u)}
$$

where, as usual,

$$
4 \pi Y_{\ell, m}^{*}\left(\hat{a}^{*}\right) \mathcal{K}_{\mathrm{df}, 3 ; p, \ell, m ; k, \ell^{\prime}, m^{\prime}}^{(2, u, u)} Y_{\ell^{\prime}, m^{\prime}}\left(\hat{a}^{\prime *}\right)=\left.\mathcal{K}_{\mathrm{df}, 3}^{(2, u, u)}\left(\vec{p}, \vec{a}, \vec{k}, \vec{a}^{\prime}\right)\right|_{a^{*}=q_{p}^{*}, a^{\prime *}=q_{k}^{*},\{\vec{p}, \vec{k}\} \in(2 \pi / L) \mathbb{Z}^{3}} .
$$

We next derive an identity for $A_{L}^{\prime(2, u)}$, which is defined in Eqs. 5.111 5.113. The basic approach is our standard move of replacing the sum over $\vec{p}$ with a $\widetilde{\mathrm{PV}}$ integral and a sumintegral difference, the latter giving rise to a factor of $F$. However, the presence of the switch state introduces new features compared to previous applications, so we work through the steps in some detail.

We first introduce the fully-integrated counterpart to $A_{L}^{\prime(2, u)}$, which we call $A^{\prime(2, u)}$. It is defined exactly as for $A_{L}^{\prime(2, u)}$ [Eqs. 5.111 5.113) and Fig. 5.11 b] except that the sum over $\vec{p}$ is replaced by a $\widetilde{P V}$-integral. This is the first example of an infinite-volume quantity with multiple $\widetilde{\mathrm{PV}}$-integrals. As we have already mentioned, a consequence of our nonstandard regulator is that the order of integration is important. In the definition of $A_{L}^{\prime(2, u)}$, the integral 
over $\vec{k}$ is done last. The difference between the two quantities can be written as

$$
\begin{aligned}
& A_{L}^{\prime(2, u)}\left(\vec{k}, a^{\prime}\right)-A^{\prime(2, u)}\left(\vec{k}, a^{\prime}\right)= \\
& \quad\left[\frac{1}{L^{3}} \sum_{\vec{p}}-\widetilde{\mathrm{PV}} \int_{\vec{p}}\right] A^{\prime(1, u)}(\vec{p}, k) \frac{H(\vec{k}) \Delta(P-p-k)}{2 \omega_{p}} i \mathcal{K}_{2 ; \mathrm{off}}\left(k, P-p-k, a^{\prime}\right),
\end{aligned}
$$

where $k=\left[\omega_{k}, \vec{k}\right]$. To obtain this form we have used the fact that $A^{\prime(1, u)}(\vec{p}, k)$ [defined in Eq. [5.88] is a smooth function of $\vec{p}$, so that the only singularity in $p$ comes from the switch state. Also, we have done the $p^{0}$ integral and kept only the particle pole, since other poles give non-singular contributions which have vanishing sum-integral differences. Finally, we have added in the cut-off function $H(\vec{k})$, which is allowed since it does not change the singularity.

To use the sum-integral identity, we need to expand $A^{\prime(1, u)}(\vec{p}, k)$ in spherical harmonics with respect to $\vec{p}^{*}$, i.e. treat $k$ as the spectator and boost to the CM frame of the other two particles [with boost velocity $\left.-(\vec{P}-\vec{k}) /\left(E-\omega_{k}\right)\right]$. This is different from the expansion used earlier, in Eq. 5.90, where $p$ was treated as the spectator. Thus we define [see Fig. 5.13a]

$$
A_{\ell, m}^{\prime(1, s)}\left(\vec{k}, p^{*}\right) \sqrt{4 \pi} Y_{\ell, m}\left(\hat{p}^{*}\right) \equiv A^{\prime(1, u)}\left(\vec{p},\left[\omega_{k}, \vec{k}\right]\right)
$$

where the superscript " $s$ " indicates that the particle carrying the momentum singled out by the coordinate system, here $\vec{k}$, is one of those scattered by the $\mathcal{K}_{2}$ inside $A^{\prime(1, u)}$. We stress that $A_{\ell, m}^{\prime(1, s)}$ and $A_{\ell, m}^{\prime(1, u)}$ are different expansions of the same underlying function-we are just using different coordinate systems. We also note that the boost defining $\vec{p}^{*}$ is well defined because of the presence of $H(\vec{k})$.

As a final step, we must also decompose the off-shell two-particle K-matrix into spherical harmonics:

$$
4 \pi Y_{\ell, m}^{*}\left(\hat{p}^{*}\right) \mathcal{K}_{2 ; \mathrm{off} ; \ell, m ; \ell^{\prime}, m^{\prime}}\left(\vec{k}, p^{*}, a^{\prime *}\right) Y_{\ell^{\prime}, m^{\prime}}\left(\hat{a}^{\prime *}\right) \equiv \mathcal{K}_{2 ; \mathrm{off}}\left(\left[\omega_{p}, \vec{p}\right], P-p-k,-\left[\omega_{a^{\prime}}, \vec{a}^{\prime}\right]\right),
$$


This allows us to write

$$
\begin{aligned}
& A_{L ; k, \ell^{\prime}, m^{\prime}}^{\prime(2, u)}-A_{k, \ell^{\prime}, m^{\prime}}^{\prime(2, u)}= \\
& {\left[\frac{1}{L^{3}} \sum_{\vec{p}}-\widetilde{\mathrm{PV}} \int_{\vec{p}}\right] A_{\ell_{1}, m_{1}}^{(1, s)}\left(\vec{k}, p^{*}\right) \frac{i 4 \pi Y_{\ell_{1}, m_{1}}\left(\hat{p}^{*}\right) H(\vec{k}) Y_{\ell_{2}, m_{2}}^{*}\left(\hat{p}^{*}\right)}{2 \omega_{p} 2 \omega_{P-p-k}\left(E-\omega_{k}-\omega_{p}-\omega_{p k}\right)} i \mathcal{K}_{2 ; \mathrm{off} ; \ell_{2}, m_{2} ; \ell^{\prime}, m^{\prime}}\left(\vec{k}, p^{*}, q_{k}^{*}\right),}
\end{aligned}
$$

where we have explicitly pulled out the particle pole in the $P-p-k$ propagator, since the remainder is non-singular. This has the form for which we can apply the sum integral identity, from which we deduce

$$
A_{L}^{\prime(2, u)}=A^{\prime(2, u)}+2 A^{\prime(1, s)} i F i \mathcal{K}_{2},
$$

where the on-shell matrix form of $A^{\prime(1, s)}$ is

$$
A_{p, \ell, m}^{\prime(1, s)}=A_{\ell, m}^{\prime(1, s)}\left(\vec{p}, q_{p}^{*}\right) \text { with } \vec{p} \in(2 \pi / L) \mathbb{Z}^{3} .
$$

The factor of two in 5.144 appears because $F$ contains a symmetry factor of $1 / 2$ which is absent in the switch-state contribution.

The new quantity $2 A^{\prime(1, s)}$ will later be combined with $A^{\prime(1, u)}$ in order to form an object which, aside from one subtlety, is symmetric under particle interchange. To understand this point, first observe that there are three independent ways that the external momenta can be assigned to the diagram: (i) $\vec{p}$ is the spectator with $\vec{k}$ one of the scattered pair (giving $\left.A^{\prime(1, u)}\right)$, (ii) $\vec{p}$ is one of the scattered pair with $\vec{k}$ the spectator (giving $A^{(1, s)}$ ), and (iii) $\vec{p}$ and $\vec{k}$ form the scattered pair. This is illustrated in Fig. 5.13 b. For the symmetry to hold we must sum these three with equal weights: (i)+(ii)+(iii) 26 This differs from the combination that arises naturally in our derivation, (i) $+2($ ii). It turns out, however, that we can replace 2 (ii) with (ii)+(iii), and thus obtain a truely symmetric combination. We do this repeatedly below, and thus explain here the justification for this change.

Momentum assignment (iii) leads to a quantity we call $A^{\prime(1, \widetilde{s})}$ that is related to $A^{\prime(1, s)}$ as follows:

$$
A_{p, \ell, m}^{(1, \widetilde{s})}=(-1)^{\ell} A_{p, \ell, m}^{\prime(1, s)}
$$

\footnotetext{
${ }^{26}$ We stress that here we are discussing on-shell quantities; the symmetry cannot hold if one of the particles is off shell.
} 
This is because the assignments (iii) and (ii) differ simply by the interchange of the two particles that have been boosted to their CM frame. (These are the particles with momenta $k$ and $P-p-k$.) This interchange is the same as a parity tranform in the CM frame, leading to the result 5.146 . We also note that $A_{p, \ell, m}^{(1, u)}$ is only non-vanishing for even $\ell$ given the symmetry of $\mathcal{K}_{2}$. Thus the desired combination

$$
A_{p, \ell, m}^{\prime(1)} \equiv A_{p, \ell, m}^{(1, u)}+A_{p, \ell, m}^{(1, s)}+A_{p, \ell, m}^{\prime(1, \widetilde{s})}
$$

satisfies

$$
A_{p, \ell, m}^{\prime(1)}= \begin{cases}A_{p, \ell, m}^{\prime(1, u)}+2 A_{p, \ell, m}^{(1, s)} & \ell \text { even }, \\ 0 & \ell \text { odd } .\end{cases}
$$

This means that we can make the replacements

$$
2 A_{p, \ell, m}^{\prime(1, s)} \longrightarrow A_{p, \ell, m}^{\prime(1, s)}+A_{p, \ell, m}^{\prime(1, \widetilde{s})} \text { and } A_{p, \ell, m}^{\prime(1, u)}+2 A_{p, \ell, m}^{\prime(1, s)} \longrightarrow A_{p, \ell, m}^{\prime(1)}
$$

as long as only even values of $\ell$ contribute.

To see that only even values of $\ell$ contribute, first recall from Eq. 5.144 that $A^{\prime(1, s)}$ is connected by an $F$ to a factor of $\mathcal{K}_{2}$. Next, note that the symmetry of $\mathcal{K}_{2}$ implies that only even angular momenta appear in its expansion. Finally, use the result Eq. D.11 in Appendix $\mathrm{D}$ that $F_{p, \ell, m ; k \ell^{\prime}, m^{\prime}}$ vanishes if $\ell+\ell^{\prime}$ is odd. Together these imply that, since $\ell^{\prime}$ is even, $\ell$ is also.

It turns out that, throughout the derivation, " $(s)$ " quantities always appear opposite those with a " $(u)$ " superscript. The latter always have the requisite symmetry so that only even angular momenta contribute. Consequently, by the argument just given, we can always replace $2(s)$ with $(s)+(\widetilde{s})$. For the sake of brevity, we do not do this explicitly, but keep in mind that this replacement is allowed. At the end of the derivation, once we have summed contributiions from any number of switches, we make the replacement explicit so as to allow symmetrization.

The identity for $A_{L}^{(2, u)}$ is derived in exactly the same way as that for $A_{L}^{(2, u)}$ and we simply state the result:

$$
A_{L}^{(2, u)}=A^{(2, u)}+i \mathcal{K}_{2} i F 2 A^{(1, s)} .
$$

Here $A_{p, \ell^{\prime}, m^{\prime}}^{(2, u)}$ and $A^{(1, s)}$ are the left-right "reflections" of the corresponding $A^{\prime}$ quantities. 
(a)

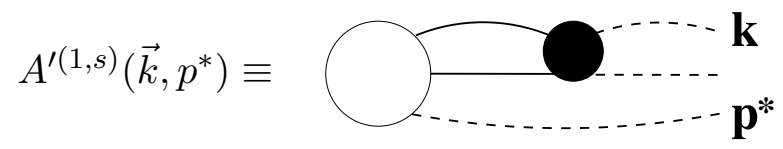

(b)

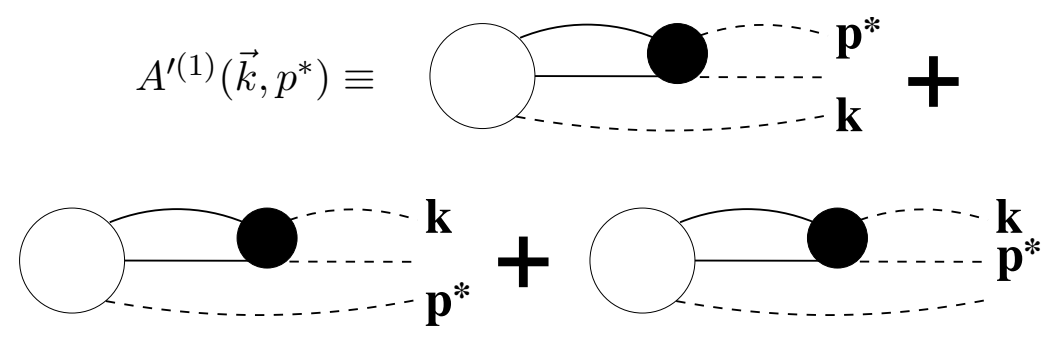

Figure 5.13: (a) Definition of $A^{\prime(1, s)}$, in which the momentum singled out by the coordinate system (here $\vec{k}$ ) is that of a particle that scatters. (b) Definition of the symmetrized quantity $A^{\prime(1)}$.

Finally we consider $C_{L, 0 F}^{(2)}$, defined in Eq. 5.118 and Fig. 5.11. Our aim is to determine the finite-volume residue that results when we convert the two momentum sums into integrals. The two-particle loops are both rendered non-singular by the $\widetilde{\mathrm{PV}}$ integrals over $a$ and $a^{\prime}$, so the only singularity is that in $\Delta(P-p-k)$. To isolate this, both $p^{0}$ and $k^{0}$ integrals must circle the particle poles. (If other poles are encircled in either integral, the remaining summand is non-singular and both sums can be immediately changed to integrals.) We then have to choose which sum to evaluate first. Our convention, here and below, is to work from left to right. Thus we first convert the sum over $\vec{p}$ into an integral plus an $F$ term. The detailed steps are exactly as for $A_{L}^{(2, u)}$, except that here we have $A^{(1, u)}$ on the right rather than $\mathcal{K}_{2}$. For the contribution in which $\vec{p}$ is integrated, there are no more singularities, so the sum over $\vec{k}$ can be converted directly into an integral. For the $F$-term, however, the sum over $\vec{k}$ must remain. The result of this analysis is that

$$
C_{L, 0 F}^{(2)}=C_{\infty}^{(2)}+2 A^{\prime(1, s)} \frac{i F}{2 \omega L^{3}} A^{(1, u)}
$$


where $C_{\infty}^{(2)}$ is the infinite-volume version of the single-switch correlator:

$$
\begin{aligned}
C_{\infty}^{(2)}=\frac{1}{4} \widetilde{\mathrm{PV}} \int_{\vec{k}} \widetilde{\mathrm{PV}} \int_{\vec{p}} \widetilde{\mathrm{PV}} \int_{a, a^{\prime}} \int_{p^{0}} \int_{k^{0}} \sigma(p, a) \Delta(a) \Delta(P-p-a) \Delta(p) i \mathcal{K}_{2 ; \mathrm{off}}(a, P-p-a,-k) \\
\quad \times \Delta(P-p-k) i \mathcal{K}_{2 ; \text { off }}\left(P-p-k, p,-a^{\prime}\right) \Delta\left(a^{\prime}\right) \Delta\left(P-k-a^{\prime}\right) \Delta(k) \sigma^{\dagger}\left(k, a^{\prime}\right) .
\end{aligned}
$$

The factor of $1 /\left(2 \omega L^{3}\right)$ in the last term in Eq. 5.151) arises because $F$ is defined to contain the contributions from only two of the three propagators in the switch state. The overall factor of 2 in this term arises because $F$ contains a symmetry factor of $1 / 2$ that is absent in the switch state. We stress here, for the final time, that the order of $\widetilde{P V}$-integration matters in the definition of this infinite-volume quantity.

Our "left to right convention" has given an asymmetric result, with $A^{\prime(1, s)}$ to the left of $A^{(1, u)}$ and no " $u F s$ " term. This lack of symmetry can, however, be corrected a posteriori, as will be explained when we consider the result from any number of switches.

Inserting the identities (5.138), (5.144), (5.150) and (5.151) into Eq. (5.119) we find the final result of this section

$$
\begin{aligned}
C_{L}^{(2)}-C_{\infty}^{(2)} & =\left(\sigma^{*}+A^{\prime(1, u)}\right)[\mathcal{A}]\left[i \mathcal{K}_{2} i G 2 \omega L^{3} i \mathcal{K}_{2}+i \mathcal{K}_{\mathrm{df}, 3}{ }^{(2, u, u)}\right][\mathcal{A}]\left(\sigma^{\dagger *}+A^{(1, u)}\right) \\
& +A^{\prime(2, u)}[\mathcal{A}]\left(\sigma^{\dagger *}+A^{(1, u)}\right)+\left(\sigma^{*}+A^{\prime(1, u)}\right)[\mathcal{A}] A^{(2, u)} \\
& +\left[2 A^{\prime(1, s)}\right] i F i \mathcal{K}_{2}[\mathcal{A}]\left(\sigma^{\dagger *}+A^{(1, u)}\right)+\left(\sigma^{*}+A^{\prime(1, u)}\right)[\mathcal{A}] i \mathcal{K}_{2} i F\left[2 A^{(1, s)}\right] \\
& +2 A^{\prime(1, s)} \frac{i F}{2 \omega L^{3}} A^{(1, u)}
\end{aligned}
$$

This is the main result of this subsection. The right-hand side is the finite-volume residue of all one-switch diagrams.

\subsubsection{Two-to-two insertions: two switches}

In this section we sum the diagrams of Fig. 5.14. These are all diagrams that have two switches in the pair that is scattered. We denote the sum of all such diagrams by $C_{L}^{(3)}$. Throughout this section we refer to leftmost (rightmost) triplet of propagators, at the point where the scattering pair changes, as the left (right) switch state. We label the three different spectator momenta $p, r$ and $k$, as shown in the figure. 


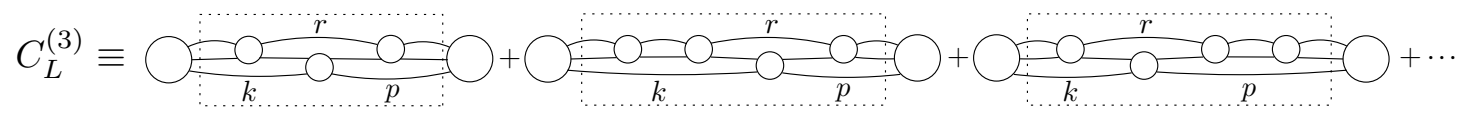

Figure 5.14: Subset of finite-volume correlator diagrams containing only two-to-two insertions and with two switches in the scattered pair.

We provide a detailed analysis of two-switch diagrams before analyzing diagrams with any number of switches for two reasons. First, a new type of intermediate quantity with finite-volume dependence arises at this order. This is $\mathcal{K}_{3, L}^{(3, u, u)}$, a contribution to threeto-three scattering. Second, a number of new complications enter at this stage with the derivation of identities relating the intermediate quantities (with $L$ subscripts) to infinitevolume quantities. We think it clearer to analyze these in isolation before generalizing to all orders.

As in the previous sections we evaluate $p^{0}, r^{0}$ and $k^{0}$ integrals and then substitute the identity of Eq. (5.75) for all two-particle loops for which the spectator is on shell. There are three locations where insertions of $F$ can appear: to the left of the left switch state, between switch states, and to the right of the right switch state. We define eight different classes of terms, based on whether or not at least one $F$ insertion appears in each of the three locations:

$$
\begin{aligned}
& \text { (1) } F, F, F \quad(2)-, F, F, \quad(3)-,-, F, \text { (4) } F, F,-, \\
& \text { (5) } F,-,-(6)-, F,-, \quad(7) F,-, F \quad(8)-,-,- \text {. }
\end{aligned}
$$

For example in class (2) there is at least one insertion to the right of the right switch and between the switches, but no insertion to the left of the left switch.

Using the methods of the previous subsections, it is straightforward to obtain the results 
from these classes. We find

$$
\begin{aligned}
C_{L}^{(3)} & =\left(\sigma^{*}+A^{\prime(1, u)}\right)[\mathcal{A}] i \mathcal{K}_{3}^{(2, u, u)}[\mathcal{A}] i \mathcal{K}_{3}^{(2, u, u)}[\mathcal{A}]\left(\sigma^{\dagger *}+A^{(1, u)}\right) \\
& +A_{L}^{(2, u)}[\mathcal{A}] i \mathcal{K}_{3}^{(2, u, u)}[\mathcal{A}]\left(\sigma^{\dagger *}+A^{(1, u)}\right)+A_{L}^{(3, u)}[\mathcal{A}]\left(\sigma^{\dagger *}+A^{(1, u)}\right) \\
& +\left(\sigma^{*}+A^{\prime(1, u)}\right)[\mathcal{A}] i \mathcal{K}_{3}^{(2, u, u)}[\mathcal{A}] A_{L}^{(2, u)}+\left(\sigma^{*}+A^{\prime(1, u)}\right)[\mathcal{A}] A_{L}^{(3, u)} \\
& +A_{L}^{\prime(2, u)}[\mathcal{A}] A_{L}^{(2, u)}+\left(\sigma^{*}+A^{\prime(1, u)}\right)[\mathcal{A}] i \mathcal{K}_{3, L}^{(3, u, u)}[\mathcal{A}]\left(\sigma^{\dagger *}+A^{(1, u)}\right)+C_{L, 0 F}^{(3)} .
\end{aligned}
$$

Here the eight terms are the results, in turn, from the eight classes of contribution identified above. The four new quantities appearing in Eq. 5.155 are $A_{L}^{\prime(3, u)}, A_{L}^{(3, u)}, C_{L, 0 F}^{(3)}$ and $\mathcal{K}_{3, L}^{(3, u, u)}$. These are defined as (see also Fig. 5.15 )

$$
A_{L ; p, \ell^{\prime}, m^{\prime}}^{\prime(3, u)} \equiv A_{L ; \ell^{\prime}, m^{\prime}}^{\prime(3, u)}\left(\vec{p}, q_{p}^{*}\right) \text { with } \vec{p} \in(2 \pi / L) \mathbb{Z}^{3}
$$

where

$$
\begin{aligned}
& A_{L ; \ell^{\prime}, m^{\prime}}^{(3, u)}\left(\vec{p}, a^{*}\right) \sqrt{4 \pi} Y_{\ell^{\prime}, m^{\prime}}\left(\hat{a}^{*}\right) \equiv \\
& \frac{1}{2} \frac{1}{L^{6}} \sum_{\vec{k}, \vec{p}} \widetilde{\mathrm{PV}} \int_{a^{\prime}} \int_{k_{0}} \int_{r^{0}} \sigma\left(k, a^{\prime}\right) \Delta\left(a^{\prime}\right) \Delta\left(P-k-a^{\prime}\right) i \mathcal{K}_{2 ; \text { off }}\left(a^{\prime}, P-k-a^{\prime},-r\right) \Delta(k) \Delta(P-r-k) \\
& \times i \mathcal{K}_{2 ; \text { off }}(k, P-k-r,-p) \Delta(r) \Delta(P-p-r) i \mathcal{K}_{2 ; \text { off }}(r, P-p-r,-a) \text {, }
\end{aligned}
$$

with $A_{L ; k, \ell, m}^{(3, u)}$ defined analogously,

$$
\begin{aligned}
& C_{L, 0 F}^{(3)} \equiv \frac{1}{4} \frac{1}{L^{9}} \sum_{\vec{k}, \vec{p}, \vec{r}} \widetilde{\mathrm{PV}} \int_{a^{\prime}} \widetilde{\mathrm{PV}} \int_{a} \int_{k^{0}} \int_{p^{0}} \int_{r^{0}} \\
& \times \sigma\left(k, a^{\prime}\right) \Delta\left(a^{\prime}\right) \Delta\left(P-k-a^{\prime}\right) i \mathcal{K}_{2 ; \mathrm{off}}\left(a^{\prime}, P-k-a^{\prime},-r\right) \Delta(k) \Delta(P-k-r) i \mathcal{K}_{2 ; \mathrm{off}}(k, P-r-k,-p) \\
& \quad \times \Delta(r) \Delta(P-p-r) i \mathcal{K}_{2 ; \mathrm{off}}(k, P-p-r,-a) \Delta(p) \Delta(P-p-a) \Delta(a) \sigma^{\dagger}(p, a), \quad(5.158)
\end{aligned}
$$

and finally

$$
\mathcal{K}_{3, L ; k, \ell^{\prime}, m^{\prime} ; p, \ell, m}^{(3, u, u)} \equiv \mathcal{K}_{3, L ; \ell^{\prime}, m^{\prime} ; \ell, m}^{(3, u, u)}\left(\vec{k}, q_{k}^{*}, \vec{p}, q_{p}^{*}\right) \text { with } \vec{p}, \vec{k} \in(2 \pi / L) \mathbb{Z}^{3}
$$

with

$$
\begin{aligned}
& 4 \pi Y_{\ell^{\prime}, m^{\prime}}^{*}\left(\hat{a}^{\prime *}\right) i \mathcal{K}_{3, L ; \ell^{\prime}, m^{\prime} ; \ell, m}^{(3, u, u)}\left(\vec{k}, a^{\prime *}, \vec{p}, a^{*}\right) Y_{\ell, m}\left(\hat{a}^{*}\right) \equiv \frac{1}{L^{3}} \sum_{\vec{r}} \int_{r^{0}} i \mathcal{K}_{2 ; \mathrm{off}}\left(a^{\prime}, P-k-a^{\prime},-r\right) \\
& \quad \times \Delta(P-k-r) \Delta(r) i \mathcal{K}_{2 ; \mathrm{off}}(k, P-k-r,-p) \Delta(P-p-r) i \mathcal{K}_{2 ; \mathrm{off}}(r, P-p-r,-a) .
\end{aligned}
$$


(a)

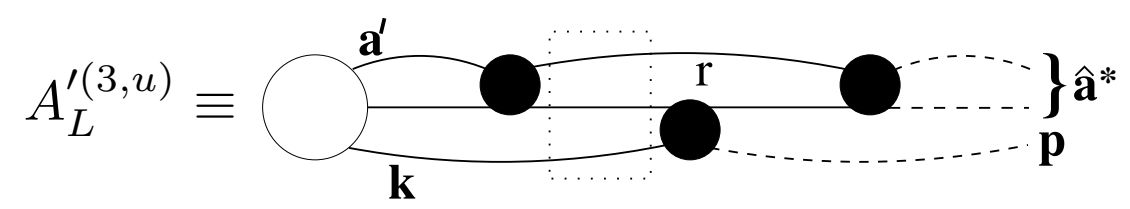

(b)

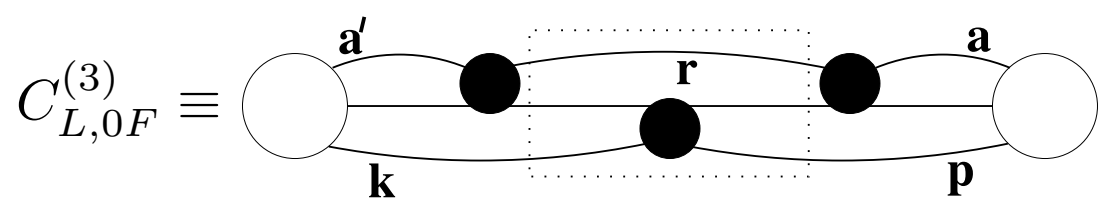

$(c)$

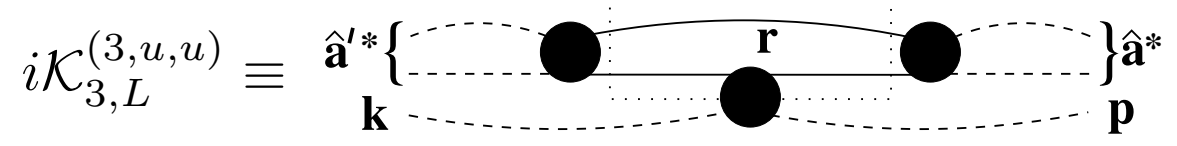

Figure 5.15: (a) Definition of $A_{L}^{\prime(3, u)}$. The dotted rectangle contains momenta which are summed; thus only the leftmost two-particle loop is integrated. (b) Definition of $C_{L, 0 F}^{(3)}$, which has two integrated and three summed loop momenta. (c) Definition of $\mathcal{K}_{3, L}^{(3, u, u)}$, which has a single summed momentum. 
To obtain these results we have summed $B_{2}$ kernels into two-particle K-matrices, and used the fact that $[\mathcal{A}]$ amputates and puts on-shell both factors adjacent to it.

We now derive identities relating the quantities $A_{L}^{(3, u)}, A_{L}^{(3, u)}, C_{L, 0 F}^{(3)}$ and $\mathcal{K}_{3, L}^{(3, u, u)}$ to infinite-volume observables. We begin with $A_{L}^{\prime(3, u)}$ (see Fig. 5.15 a), and work from left to right converting sums into integrals. The $\vec{k}$ sum leaves a finite-volume residue because of the singular propagator $\Delta(P-k-r)$, while the $\vec{r}$ sum leaves a residue because of $\Delta(P-p-r)$. The infinite-volume quantity that results, which we call $A^{\prime(3, u)}$, is thus given by the same expressions as Eqs. 5.156 and $\sqrt[5.157]{ }$ except that $\sum_{\vec{k}, \vec{p}}$ is replaced by $\widetilde{\mathrm{PV}} \int_{\vec{k}} \widetilde{\mathrm{PV}} \int_{\vec{p}}$ in Eq. (5.157). The finite-volume residues can be obtained using the same argumentation as for $A_{L}^{\prime(2, u)}$ in the previous subsection. The result is

$$
A_{L}^{\prime(3, u)}=A^{\prime(3, u)}+2 A^{\prime(1, s)} \frac{i F}{2 \omega L^{3}} i \mathcal{K}_{3}^{(2, u, u)}+2 A^{\prime(2, s)} \frac{i F}{2 \omega L^{3}} i \mathcal{K}_{2}
$$

Note the superscripts " $s$ " on the $A_{L}$ 's and the factors of 2 due to the missing symmetry factor at the switch states. The new quantity $A^{\prime(2, s)}$ is simply $A^{\prime(2, u)}$ expressed in the alternative coordinate system, just as in the definition of $A^{\prime(1, s)}$, Eq. (5.174). We can now use the result from the previous subsection for $\mathcal{K}_{3}^{(2, u, u)}$, Eq. 5.138, to obtain the desired identity

$$
A_{L}^{\prime(3, u)}=A^{\prime(3, u)}+2 A^{\prime(2, s)} i F i \mathcal{K}_{2}+2 A^{\prime(1, s)} \frac{i F}{2 \omega L^{3}} i \mathcal{K}_{2} i G 2 \omega L^{3} i \mathcal{K}_{2}+2 A^{\prime(1, s)} \frac{i F}{2 \omega L^{3}} i \mathcal{K}_{\mathrm{df}, 3}^{(2, u, u)}
$$

This derivation naturally lends itself to a recursive extension to higher order, one that we will explain in the next subsection.

The result for $A_{L}^{(3, u)}$ is given simply by reversing the order of factors in each term:

$$
A_{L}^{(3, u)}=A^{(3, u)}+i \mathcal{K}_{2} i F 2 A^{(2, s)}+i \mathcal{K}_{2} i G i \mathcal{K}_{2} i F 2 A^{(1, s)}+i \mathcal{K}_{\mathrm{df}, 3}^{(2, u, u)} \frac{i F}{2 \omega L^{3}} 2 A^{(1, s)} .
$$

We next consider $C_{L, 0 F}^{(3)}$. Working from left to right we obtain the infinite-volume quantity plus one finite-volume residue from the $\vec{k}$ sum and another from the $\vec{r}$ sum. Following our by now standard manipulations, this leads to

$$
C_{L, 0 F}^{(3)}=C_{\infty}^{(3)}+2 A^{\prime(1, s)} \frac{i F}{2 \omega L^{3}} A_{L}^{(2, u)}+2 A^{\prime(2, s)} \frac{i F}{2 \omega L^{3}} A^{(1, u)}
$$


Here $C_{\infty}^{(3)}$ is defined as in Eq. 5.158$)$ except that the momentum sums are replaced by the ordered integrals $\widetilde{\mathrm{PV}} \int_{\vec{p}} \widetilde{\mathrm{PV}} \int_{\vec{r}} \widetilde{\mathrm{PV}} \int_{\vec{k}}$. Note that $A_{L}^{(2, u)}$ still contains a momentum sum, but we can obtain a complete decomposition using Eq. 5.150 from the previous subsection. This leads to

$$
\begin{aligned}
C_{L, 0 F}^{(3)}-C_{\infty}^{(3)}=2 A^{\prime(1, s)} \frac{i F}{2 \omega L^{3}} i \mathcal{K}_{2} i F 2 & A^{(1, s)} \\
& +2 A^{\prime(1, s)} \frac{i F}{2 \omega L^{3}} A^{(2, u)}+2 A^{\prime(2, s)} \frac{i F}{2 \omega L^{3}} A^{(1, u)} .
\end{aligned}
$$

We are thus left with $\mathcal{K}_{3, L}^{(3, u, u)}$ [Eqs. 5.159 5.160 and Fig. 5.15]. As always, our method is to replace sums with integrals while keeping track of finite-volume remainders. The analysis is shown diagrammatically in Fig. 5.16. The first step is to do the $r^{0}$ integral. Singular terms occur only if the contour circles the $r^{0}=\omega_{r}$ pole; for the remainder we can replace the sum over $\vec{r}$ with an integral (giving the last term on the right-hand side in Fig. 5.16a). Thus to study the singular terms we can replace $\Delta(r)$ with $1 /\left(2 \omega_{r}\right)$ and set $r=\left[\omega_{r}, \vec{r}\right]$ (indicated by the dashed top line in the figure). The sum over $\vec{r}$ runs over two potential singularities, one in $\Delta(P-k-r)$ and the other in $\Delta(P-p-r)$. To use the sum-minus-integral identity, we must pull out the double singularity (the first term on the right-hand side in Fig. 5.16 a), leaving a remainder with at most single singularities. To do so we follow the analysis of the previous subsection [see Eqs. [5.120.5.138] ], applied separately to the two propagators, both of which are sandwiched between factors of $\mathcal{K}_{2}$. This analysis can be applied independently to the contributions associated with each propagator, with each separated into into an on-shell singular part and a divergence-free quantity. This leads to the decomposition

$$
\begin{aligned}
i \mathcal{K}_{3, L}^{(3, u, u)}=i \mathcal{K}_{2} i G i \mathcal{K}_{2} i G\left[2 \omega L^{3}\right] i \mathcal{K}_{2}+i \mathcal{K}_{2} i G i \mathcal{K}_{\mathrm{df}, 3}^{(2, u, u)} & \\
& +i \mathcal{K}_{\mathrm{df}, 3}^{(2, u, u)}\left[1 /\left(2 \omega L^{3}\right)\right] i G\left[2 \omega L^{3}\right]+\mathcal{R}
\end{aligned}
$$

where the first three terms correspond to the first three terms on the right-hand side of Fig. 5.16 27 while $\mathcal{R}$ is the sum of the last two diagrams in the figure. The only properties

\footnotetext{
${ }^{27}$ The appearance of $\left[2 \omega L^{3}\right]$ and its inverse in the third but not the second term is due to the facts that $\left[1 /\left(2 \omega L^{3}\right)\right]$ appears on the right in the definition of $G$, Eq. 5.21 , and that $\left[2 \omega L^{3}\right]$ does not commute with
} $G$. 
(a)

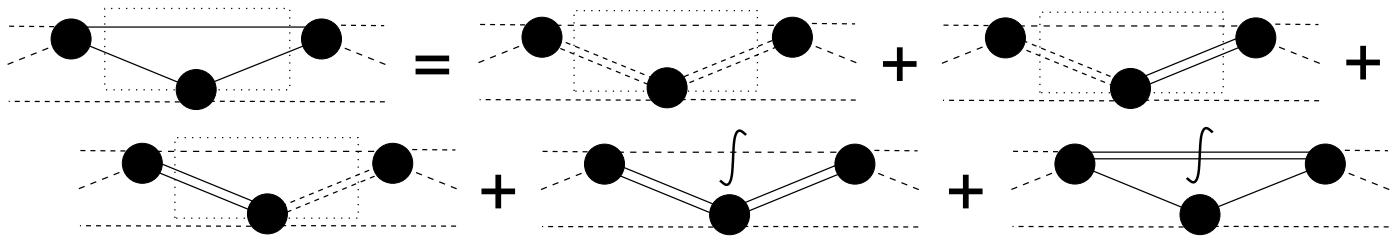

(b)

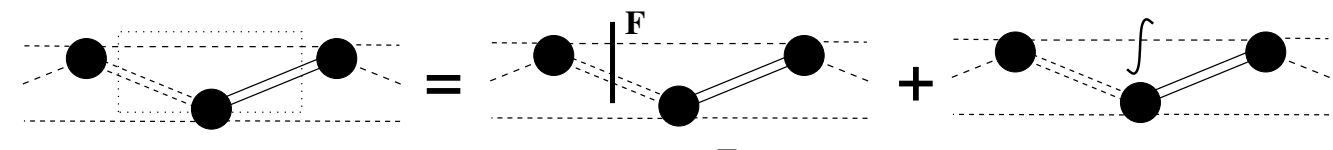

(c)
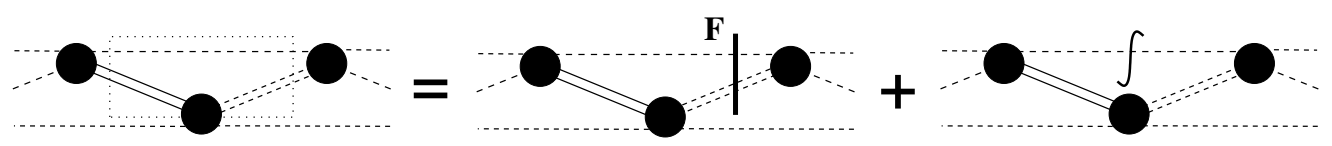

Figure 5.16: Decomposition of $\mathcal{K}_{3, L}^{(3, u, u)}$. See Fig. 5.15 (c) for momentum labels. Double solid lines indicate non-singular terms. On the top line these come from the $r^{0}$ contour circling poles other than the single-particle pole, while for the diagonal lines in the switch state the notation is as in Fig. 5.12. Double dashed lines represent the singular quantity $G^{b}$ sandwiched between on-shell amplitudes, as in Fig. 5.12. The single dashed line within the loop (top propagator) indicates the on-shell propagator factor $1 /\left(2 \omega_{r}\right)$. (a) Initial decomposition. Loop momenta inside dotted boxes are summed, while those not in a box are integrated. (b) and (c): Use of the sum-minus-integral identity, as indicated by the vertical bars and factors of $F$, leaving a remainder which is integrated. The vertical bar crosses the two propagators whose momenta are projected on-shell by $F$, so that the uncrossed propagator is the spectator. $\mathcal{K}_{\mathrm{df}, 3}^{(3, u, u)}$ is given by the sum of the four terms containing loop integrals (two in (a) and one each in (b) and (c). 
of $\mathcal{R}$ that we will need are that it is an infinite-volume quantity (since the sum over $\vec{r}$ which it contains can be replaced by an integral) and that it is a smooth function of its arguments. An explict form for $\mathcal{R}$ is not needed-Eq. (5.166) serves as sufficient definition since all terms other than $\mathcal{R}$ are known.

As noted above, we can use the sum-minus-integral identity on the two terms in Eq. (5.166) containing a single factor of $G$. Unpacking our abbreviated notation, the first such term can be written

$$
\begin{aligned}
& {\left[i \mathcal{K}_{2} i G i \mathcal{K}_{\mathrm{df}, 3}^{(2, u, u)}\right]_{p, \ell, m ; k, \ell^{\prime}, m^{\prime}}=} \\
& \left.\widetilde{\mathrm{PV}} \int_{\vec{r}} \frac{1}{2 \omega_{r}} i \mathcal{K}_{2 ; \ell, m ; \ell_{1}, m_{1}}(\vec{p}) i G_{\ell_{1}, m_{1} ; \ell_{2}, m_{2}}^{b}(\vec{p}, \vec{r}) i \mathcal{K}_{d f, 3 ; \ell_{2}, m_{2} ; \ell^{\prime} m^{\prime}}^{(2, u, u}, q_{r}^{*}, \vec{k}, q_{k}^{*}\right) \\
& +\left[\frac{1}{L^{3}} \sum_{\vec{r}}-\widetilde{\mathrm{PV}} \int_{\vec{r}} i \mathcal{K}_{2 ; \ell, m ; \ell_{1}, m_{1}}(\vec{p})\left(\frac{r^{*}}{q_{p}^{*}}\right)^{\ell_{1}} \frac{i 4 \pi Y_{\ell_{1}, m_{1}}\left(\hat{r}^{*}\right) H(\vec{p}) H(\vec{r}) Y_{\ell_{2}, m_{2}}^{*}\left(\hat{p}^{*}\right)}{2 \omega_{r} 2 \omega_{p r}\left(E-\omega_{p}-\omega_{r}-\omega_{p r}\right)}\left(\frac{p^{*}}{q_{r}^{*}}\right)^{\ell_{2}}\right. \\
& \times i \mathcal{K}_{d f, 3 ; r, \ell_{2}, m_{2} ; k, \ell^{\prime} m^{\prime}}^{(2, u, u)}
\end{aligned}
$$

The integral (the last term in Fig. 5.16p) is combined with the corresponding integral from the third term in Eq. 5.166 (the last term in Fig. 5.16 c), and with $\mathcal{R}$, to define $\mathcal{K}_{d f, 3}^{(3, u, u)}$. This is the two-switch contribution to the continuum divergence-free amplitude. The sumintegral difference requires some adjustments to allow the use of our identity. First we make the substitution

$$
\begin{aligned}
& \sqrt{4 \pi} Y_{\ell_{2}, m_{2}}^{*}\left(\hat{p}^{*}\right)\left(p^{*} / q_{r}^{*}\right)^{\ell_{2}} \mathcal{K}_{\mathrm{df}, 3 ; r, \ell_{2}, m_{2} ; k, \ell^{\prime}, m^{\prime}}^{(2, u, u)}= \\
& \sqrt{4 \pi} Y_{\ell_{2}, m_{2}}^{*}\left(\hat{p}^{*}\right)\left(p^{*} / q_{r}^{*}\right)^{\ell_{2}} \mathcal{K}_{\mathrm{df}, 3 ; \ell_{2}, m_{2} ; \ell^{\prime}, m^{\prime}}^{(2, u,}\left(\vec{r}, q_{r}^{*}, \vec{k}, q_{k}^{*}\right) \\
& \longrightarrow \sqrt{4 \pi} Y_{\ell_{2}, m_{2}}^{*}\left(\hat{p}^{*}\right) \mathcal{K}_{\mathrm{df}, 3 ; \ell_{2}, m_{2} ; \ell^{\prime}, m^{\prime}}^{(2, u, u)}\left(\vec{r}, p^{*}, \vec{k}, q_{k}^{*}\right) \equiv \mathcal{K}_{\mathrm{df}, 3 ; ; \ell^{\prime}, m^{\prime}}^{(2, u, u)}\left(\vec{r}, \vec{p}, \vec{k}, q_{k}^{*}\right)
\end{aligned}
$$

In words we are changing $q_{k}^{*} \rightarrow p^{*}$, which is allowed because the difference between the old and new forms is proportional to $p^{* 2}-q_{p}^{* 2}$, which cancels the singularity. We explain in Appendix $\mathrm{D}$ why the difference has this particular scaling. In fact, we have already made implicit use of the scaling in our on-shell identity for the sum-integral-difference, Eq. 5.70. After this change, the sum over $\ell_{2}$ and $m_{2}$ can be done, leading to a version of $\mathcal{K}_{\mathrm{df}, 3}^{(2, u, u)}$ which is off shell on the left ${ }^{28}$ At this stage we can drop the $H(\vec{r})$ factor from the summand of

\footnotetext{
${ }^{28}$ Our notation for this quantity, $\mathcal{K}_{\mathrm{df}, 3 ; ; \ell^{\prime}, m^{\prime}}^{(2, u, u)}\left(\vec{r}, \vec{p}, \vec{k}, q_{k}^{*}\right)$ indicates through the absence of a subscript
} 
the sum-minus-integral term in Eq. (5.167), since it is not needed to define the boosts, and the difference $1-H(\vec{r})$ cancels the singularity. These manipulations bring the sum-minusintegral into a form where we can apply our standard identity. In this way we find that the sum-minus-integral in Eq. (5.167) can be written

$$
i \mathcal{K}_{2} i F 2 i \mathcal{K}_{\mathrm{df}, 3}^{(2, s, u)}
$$

where $K_{\mathrm{df}, 3}^{(2, s, u)}$ is defined by re-expanding $K_{\mathrm{df}, 3}^{(2, u, u)}$ in spherical harmonics in $\vec{r}$. This is shown by the first diagram on the right-hand side in Fig. 5.16b. Specifically, the off-shell form of $K_{\mathrm{df}, 3}^{(2, u, u)}$ is expanded in harmonics

$$
\sqrt{4 \pi} Y_{\ell_{1}, m_{1}}^{*}\left(\hat{r}^{*}\right) \mathcal{K}_{\mathrm{df}, 3 ; \ell_{1}, m_{1} ; \ell^{\prime}, m^{\prime}}^{(2, s, u)}\left(\vec{p}, r^{*}, \vec{k}, q_{k}^{*}\right) \equiv \mathcal{K}_{\mathrm{df}, 3 ; ; \ell^{\prime}, m^{\prime}}^{(2, u, u)}\left(\vec{r}, \vec{p}, \vec{k}, q_{k}^{*}\right)
$$

and then put on shell and restricted to finite-volume momenta,

$$
\mathcal{K}_{\mathrm{df}, 3 ; p, \ell_{1}, m_{1} ; k, \ell^{\prime}, m^{\prime}}^{(2, s, u)} \equiv \mathcal{K}_{\mathrm{df}, 3 ; \ell_{1}, m_{1} ; \ell^{\prime}, m^{\prime}}^{(2, s, u)}\left(\vec{p}, q_{p}^{*}, \vec{k}, q_{k}^{*}\right) \quad\left[\vec{p}, \vec{k} \in(2 \pi / L) \mathbb{Z}^{3}\right]
$$

The superscript "s" once again indicates that the momentum singled-out by the coordinate system on the left, here $\vec{p}$, is one of the scattered outgoing particles.

We stress that the validity of Eq. 5.169 requires two properties of $\mathcal{K}_{\mathrm{df}, 3}^{(2, u, u)}$. First, it must be a smooth function of its arguments, for otherwise there would be additional contributions to the sum-integral difference. As discussed in the previous subsection, smoothness requires that $G$ be defined including the factors of $\left(p^{*}\right)^{\ell_{1}+\ell_{2}}$. Second, it must be divergence-free, and thus local in position-space, so that the expansion in spherical harmonics of $\hat{r}^{*}$ is convergent. This is one of the ways that our analysis forces us to use divergence-free quantities, as announced in the introduction.

The other term in Eq. (5.166) containing a single factor of $G$ can be analyzed in a similar fashion, leading to an integral plus the finite-volume residue $2 i \mathcal{K}_{\mathrm{df}, 3}^{(2, u, s)} i F i \mathcal{K}_{2}$. This is shown in Fig. 5.16. Here $\mathcal{K}_{\mathrm{df}, 3}^{(2, u, s)}$ is defined in an analogous way to $\mathcal{K}_{\mathrm{df}, 3}^{(2, s, u)}$, but with the re-expansion in new coordinates occuring for the incoming momenta. As already noted, the integral is part of the non-singular remainder which builds up $\mathcal{K}_{\mathrm{df}, 3}^{(3, u, u)}$. Combining all 
elements, we finally reach

$$
i \mathcal{K}_{3, L}^{(3, u, u)}=i \mathcal{K}_{2} i G i \mathcal{K}_{2} i G\left[2 \omega L^{3}\right] i \mathcal{K}_{2}+i \mathcal{K}_{2} i F 2 i \mathcal{K}_{\mathrm{df}, 3}^{(2, s, u)}+2 i \mathcal{K}_{\mathrm{df}, 3}^{(2, u, s)} i F i \mathcal{K}_{2}+i \mathcal{K}_{\mathrm{df}, 3}^{(3, u, u)}
$$

This has the desired form in which each term is a product of on-shell, infinite-volume quantities and kinematic factors.

The result (5.172) and the similar decompositions in Eqs. (5.162), (5.163) and (5.165) can now be substituted in Eq. (5.155) to obtain our final result for the two-switch correlator $C_{L}^{(3)}$. The result is lengthy and, at this stage, unilluminating. We hold off on making such substitutions until we are working to all orders, in the next subsection, for then the result simplifies.

\subsubsection{Two-to-two insertions: any number of switches}

In this section we sum all remaining contributions to the finite-volume correlator containing only $B_{2}$ kernels, allowing any number of switches in scattered pair.

The first step, as before, is to replace sums on two-particle loops with integrals plus factors of $F$. This leads to the appearance of $C_{L, 0 F}^{(n)}, A_{L}^{(n, u)}, A_{L}^{(n, u)}$ and $\mathcal{K}_{3, L}^{(n, u, u)}$, with $n \geq 3$, which are generalizations of the quantities found earlier. Their definitions, sketched in Fig. 5.17, are

$$
\begin{gathered}
C_{L, 0 F}^{(n)} \equiv \frac{1}{4}\left[\prod_{m=1}^{n} \frac{1}{L^{3}} \sum_{\vec{q}_{m}}\right] \widetilde{\mathrm{PV}} \int_{a} \widetilde{\mathrm{PV}} \int_{a^{\prime}}\left[\prod_{m=1}^{n} \int_{q_{m}^{0}}\right] \sigma\left(q_{1}, a\right) \Delta(a) \Delta\left(P-q_{1}-a\right) \\
\times i \mathcal{K}_{2, \mathrm{off}}\left(a, P-q_{1}-a,-q_{2}\right) \Delta\left(q_{1}\right) \Delta\left(P-q_{1}-q_{2}\right) i \mathcal{K}_{2, \mathrm{off}}\left(q_{1}, P-q_{1}-q_{2},-q_{3}\right) \times \cdots \times \\
\times \Delta\left(q_{n-1}\right) \Delta\left(P-q_{n}-q_{n-1}\right) i \mathcal{K}_{2, \mathrm{off}}\left(q_{n-1}, P-q_{n}-q_{n-1},-a^{\prime}\right) \Delta\left(q_{n}\right) \Delta\left(P-q_{n}-a^{\prime}\right) \Delta\left(a^{\prime}\right) \sigma^{\dagger}\left(q_{n}, a^{\prime}\right) \\
A_{L ; \ell^{\prime}, m^{\prime}}^{\prime(n, u)}\left(\vec{k}, a^{\prime *}\right) \sqrt{4 \pi} Y_{\ell^{\prime}, m^{\prime}}\left(\hat{a}^{\prime *}\right) \equiv \frac{1}{2}\left[\prod_{m=1}^{n-1} \frac{1}{L^{3}} \sum_{\vec{q}_{m}}\right] \widetilde{\mathrm{PV}} \int_{a}\left[\prod_{m=1}^{n-1} \int_{q_{m}^{0}}\right] \\
\times \sigma\left(q_{1}, a\right) \Delta(a) \Delta\left(P-q_{1}-a\right) i \mathcal{K}_{2, \mathrm{off}}\left(a, P-q_{1}-a,-q_{2}\right) \Delta\left(q_{1}\right) \Delta\left(P-q_{1}-q_{2}\right) i \mathcal{K}_{2, \mathrm{off}}\left(q_{1}, P-q_{1}-q_{2},-q_{3}\right) \\
\times \Delta\left(q_{2}\right) \Delta\left(P-q_{3}-q_{2}\right) \times \cdots \times i \mathcal{K}_{2, \mathrm{off}}\left(q_{n-2}, P-q_{n-2}-q_{n-1},-p\right) \\
\times \Delta\left(q_{n-1}\right) \Delta\left(P-p-q_{n-1}\right) i \mathcal{K}_{2, \mathrm{off}}\left(q_{n-1}, P-p-q_{n-1},-a^{\prime}\right)
\end{gathered}
$$


(a)

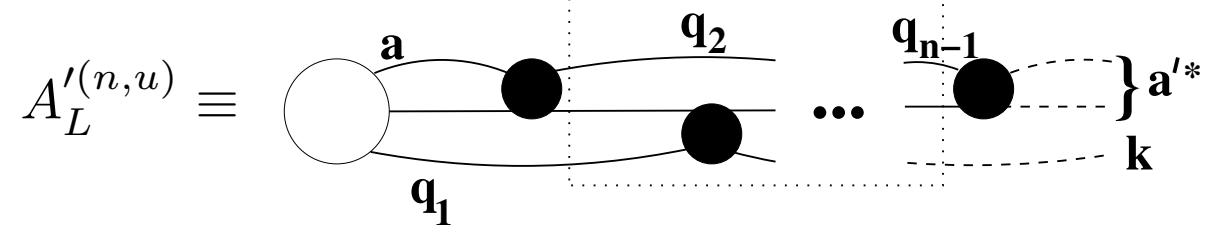

(b)

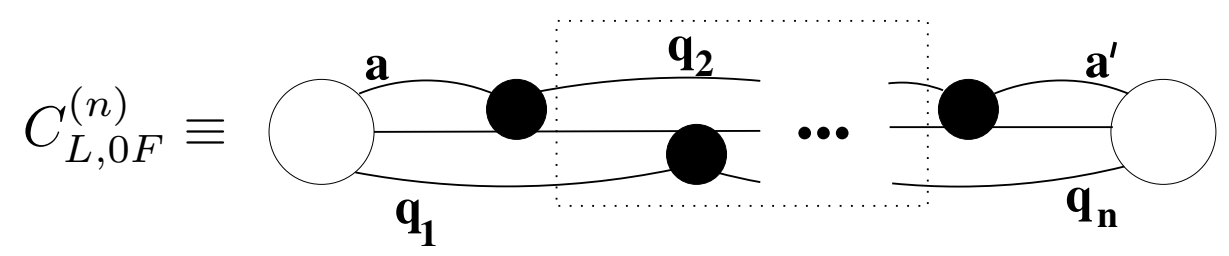

(c)

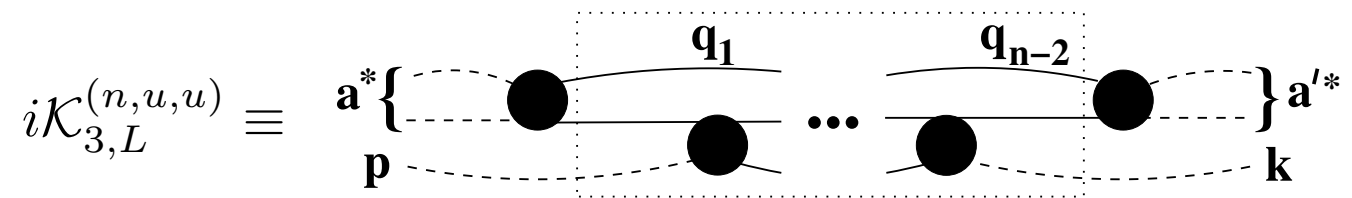

Figure 5.17: Diagrammatic definitions of (a) $A_{L}^{\prime(n, u)}$, (b) $C_{L, 0 F}^{(n)}$, and (c) $\mathcal{K}_{3, L}^{(n, u, u)}$. 
with $A_{L}^{(n, u)}$ defined analogously by reflection, and

$$
\begin{aligned}
4 \pi Y_{\ell^{\prime}, m^{\prime}}^{*}\left(\hat{a}^{*}\right) i \mathcal{K}_{3, L ; \ell^{\prime}, m^{\prime} ; \ell, m}^{(n, u, u)}\left(\vec{p}, a^{*}, \vec{k}, a^{*}\right) Y_{\ell, m}\left(\hat{a}^{\prime *}\right) \equiv\left[\prod_{m=1}^{n-2} \frac{1}{L^{3}} \sum_{\vec{q}_{m}}\right]\left[\prod_{m=1}^{n-2} \int_{q_{m}^{0}}\right] \\
\times i \mathcal{K}_{2, \mathrm{off}}\left(a, P-p-a,-q_{1}\right) \Delta\left(P-p-q_{1}\right) i \mathcal{K}_{2, \mathrm{off}}\left(p, P-p-q_{1},-q_{2}\right) \\
\times \Delta\left(q_{2}\right) \Delta\left(P-q_{1}-q_{2}\right) \times \cdots \times \Delta\left(q_{n-2}\right) \Delta\left(P-k-q_{n-2}\right) i \mathcal{K}_{2, \mathrm{off}}\left(q_{n-2}, P-p-q_{n-2},-a^{\prime}\right),
\end{aligned}
$$

The above definitions give partially off shell versions of $A_{L}^{(n, u)}$ and $\mathcal{K}_{3, L}^{(n, u, u)}$. The on-shell versions are defined as usual by

$$
\begin{gathered}
A_{L ; k, \ell^{\prime}, m^{\prime}}^{\prime(n, u)} \equiv A_{L ; \ell^{\prime}, m^{\prime}}^{(n, u)}\left(\vec{k}, q_{k}^{*}\right) \text { and } \mathcal{K}_{3, L ; p, \ell^{\prime}, m^{\prime} ; k, \ell, m}^{(n, u, j)} \equiv \mathcal{K}_{3, L ; \ell^{\prime}, m^{\prime} ; \ell, m}^{(n, u, u)}\left(\vec{p}, q_{p}^{*}, \vec{k}, q_{k}^{*}\right) \\
\text { with } \vec{p}, \vec{k} \in(2 \pi / L) \mathbb{Z}^{3},
\end{gathered}
$$

with an analogous definition for $A_{L}^{(n, u)}$.

It is simpler to write down the all-orders form of

$$
C_{L}^{\left[B_{2}\right]}=\sum_{n=0}^{\infty} C_{L}^{(n)},
$$

than it is to write down $C_{L}^{(n)}$ itself. The superscript "[ $\left.B_{2}\right]$ " here is a reminder that no $B_{3}$ kernels have yet been included. We find

$$
\begin{aligned}
C_{L}^{\left[B_{2}\right]}=\sum_{n=0}^{\infty} C_{L, 0 F}^{(n)}-(2 / 3) & \sigma^{*} \frac{i F}{2 \omega L^{3}} \sigma^{\dagger *} \\
+ & {\left[\sum_{i=0}^{\infty} A_{L}^{(i, u)}\right][\mathcal{A}]\left\{\sum_{n=0}^{\infty}\left(i \mathcal{K}_{3, L}^{(u, u)}[\mathcal{A}]\right)^{n}\right\}\left[\sum_{k=0}^{\infty} A_{L}^{(k, u)}\right] }
\end{aligned}
$$

Here we have made the definitions

$$
\begin{gathered}
A_{L}^{(0, u)} \equiv \sigma^{* \dagger}, \quad A_{L}^{(1, u)} \equiv A^{(1, u)}, \quad A_{L}^{\prime(0, u)} \equiv \sigma^{*}, \quad A_{L}^{(1, u)} \equiv A^{\prime(1, u)} \\
i \mathcal{K}_{3, L}^{(2, u, u)} \equiv i \mathcal{K}_{3}^{(2, u, u)}, \quad C_{L, 0 F}^{(0)} \equiv C_{\infty}^{(0)}, \quad C_{L, 0 F}^{(1)} \equiv C_{\infty}^{(1)}
\end{gathered}
$$

in which infinite-volume quantities are relabeled as though they have volume dependence, in order to simplify the form of the result. We have also introduced

$$
\mathcal{K}_{3, L}^{(u, u)} \equiv \sum_{n=2}^{\infty} \mathcal{K}_{3, L}^{(n, u, u)}
$$


The way in which $(5.178)$ arises should be clear by generalizing the discussion leading to the results for $C_{L}^{(0)}+C_{L}^{(1)}\left[\right.$ Eq. 5.96)], $C_{L}^{(2)}$ [Eq. 5.119] ] and $C_{L}^{(3)}$ [Eq. 5.155)] above. In words, one has endcaps, involving any number of switches, connected to any number of finitevolume three-particle scattering amplitudes with intemediate factors of $[\mathcal{A}]$. Recall that $[\mathcal{A}]$, defined in Eq. 5.95 , is closely related to the two-particle finite-volume propagator.

To bring Eq. (5.178) into a useful form we need identities relating all quantities with $L$ subscripts to infinite-volume quantities and finite-volume remainders.

We first consider $A_{L}^{\prime(n, u)}$, and for now seek only to rewrite this in terms of $\mathcal{K}_{3, L}^{(j, u, u)}$ as well as infinite-volume quantities. Our basic strategy is to move from left to right, replacing sums with integrals plus sum-integral differences. The reason for moving from left to right is that each sum then has a summand with only one singular factor, which we know how to handle. All double singularities are removed, because each sum is adjacent to an integral which removes the singularities in one of the two switch-states containing the summed coordinate.

We describe in some detail how the process works for $q_{1}$ and then state the final result. The sum over $\vec{q}_{1}$ has a potentially singular summand in the propagator $\Delta\left(P-q_{1}-q_{2}\right)$ (the singularity in $\Delta\left(P-q_{1}-a\right)$ being removed by the the $\widetilde{\mathrm{PV}}$ integral $)$. For this singularity to be present, both $q_{1}$ and $q_{2}$ must be on shell, so we must first do the $q_{1}^{0}$ and $q_{2}^{0}$ integrals and pick out the particle poles. In this singular term we can replace the sum over $\vec{q}_{1}$ with an integral plus the sum-integral difference. Generalizing the analysis given earlier, we find that the sum-integral difference gives

$$
2 A^{\prime(1, s)} \frac{i F}{2 \omega L^{3}} i \mathcal{K}_{3, L}^{(n-1, u, u)}
$$

What remains are terms involving the $\widetilde{\mathrm{PV}}$ integral over $\vec{q}_{1}$, and these can be repackaged into a quantity with exactly the form of $A_{L}^{\prime(n, u)}$ [Eq. 5.174] ] except that the sum-integral $1 / L^{3} \sum_{\vec{q}_{1}} \widetilde{\mathrm{PV}} \int_{q_{1}}$ is replaced by the four-momentum integral $\widetilde{\mathrm{PV}} \int_{q_{1}}$. In this way the finitevolume residue from the sum over $\vec{q}_{1}$ has been determined.

We now repeat this analysis for $q_{2}$, finding that the $F$ term is

$$
2 A^{(2, s)} \frac{i F}{2 \omega L^{3}} i \mathcal{K}_{3, L}^{(n-2, u, u)}
$$

while the remainder has the form of $A_{L}^{\prime(n, u)}$ with sum-integrals over both $q_{1}$ and $q_{2}$ replaced 
by integrals. Continuing this pattern, we deduce

$$
A_{L}^{\prime(n, u)}=\sum_{i=1}^{n-2} 2 A^{\prime(i, s)} \frac{i F}{2 \omega L^{3}} i \mathcal{K}_{3, L}^{(n-i, u, u)}+2 A^{\prime(n-1, s)} i F i \mathcal{K}_{2}+A^{\prime(n, u)}
$$

This result holds for $n>2$, and agrees with Eq.(5.161) for $n=3$. The infinite-volume quantity $A^{\prime(n, u)}$ is given by the same expression as $A_{L}^{\prime(n, u)}$ [Eq. 5.174 ] except that all sums are replaced with $\widetilde{\mathrm{PV}}$ integrals, with the order being

$$
\widetilde{\mathrm{PV}} \int_{q_{n}} \cdots \widetilde{\mathrm{PV}} \int_{q_{1}} \widetilde{\mathrm{PV}} \int_{a}
$$

The quantities $A^{\prime(n, s)}$ are defined in terms of $A^{\prime(n, u)}$ by changing variables exactly as for $A^{\prime(1, s)}$ [see Eq. (5.141)].

The analysis for $A_{L}^{(n, u)}$ is the mirror image of that for $A_{L}^{\prime(n, u)}$, so that the sums are now dealt with moving from right to left. The result is

$$
A_{L}^{(n, u)}=\sum_{i=1}^{n-2} i \mathcal{K}_{3, L}^{(n-i, u, u)} \frac{i F}{2 \omega L^{3}} 2 A^{(i, s)}+i \mathcal{K}_{2} i F 2 A^{(n-1, s)}+A^{(n, u)} .
$$

We treat $C_{L, 0 F}^{(n)}$ in a similar fashion. Here we can choose to work from left to right or vice versa - both choices lead to single singularities for each loop sum. As above, our convention is to work from left to right. Since the analysis follows that for $A_{L}^{\prime(n, u)}$ very closely, we simply quote the result,

$$
\begin{aligned}
C_{L, 0 F}^{(n)} & =\sum_{i=1}^{n-2} 2 A^{\prime(i, s)} \frac{i F}{2 \omega L^{3}} A_{L}^{(n-i, u)}+2 A^{\prime(n-1, s)} \frac{i F}{2 \omega L^{3}} A^{(1, u)}+C_{\infty}^{(n)}, \\
& =\sum_{i=1}^{n-1} 2 A^{\prime(i, s)} \frac{i F}{2 \omega L^{3}} A_{L}^{(n-i, u)}+C_{\infty}^{(n)} .
\end{aligned}
$$

To obtain the second form we have used $A_{L}^{(1, u)} \equiv A^{(1, u)}$. The quantity $C_{\infty}^{(n)}$ takes the same form as $C_{L, 0 F}^{(n)}$ [Eq. 5.173$]$ ] except that all sums are replaced by integrals, ordered as

$$
\widetilde{\mathrm{PV}} \int_{q_{n}} \cdots \widetilde{\mathrm{PV}} \int_{q_{1}} \widetilde{\mathrm{PV}} \int_{a} \widetilde{\mathrm{PV}} \int_{a^{\prime}}
$$

The result 5.188) is valid for $n>1$, and agrees with Eqs. (5.151) and 5.164 for $n=2$ and 3 , respectively. 
The next step towards simplifying the result for $C_{L}^{\left[B_{2}\right]}$, Eq. 5.178 , is to perform the sums over the number of switches. In particular, from Eq. (5.184) we find

$$
A_{L}^{\prime(u)} \equiv \sum_{n=1}^{\infty} A_{L}^{\prime(n, u)}=A^{\prime(u)}+2 A^{\prime(s)}\left[i F i \mathcal{K}_{2}+\frac{i F}{2 \omega L^{3}} i \mathcal{K}_{3, L}^{(u, u)}\right],
$$

where

$$
A^{\prime(u)} \equiv \sum_{n=1}^{\infty} A^{\prime(n, u)} \text { and } A^{\prime(s)} \equiv \sum_{n=1}^{\infty} A^{\prime(n, s)} .
$$

Similar definitions will be used for $A^{(u)}$ and $A^{(s)}$, and also for the amplitudes corresponding to the third choice of momentum assignments, $A^{\prime(\widetilde{s})}$ and $A^{(\widetilde{s})}$. The latter were introduced in Eq. (5.146).

An analogous result to Eq. 5.190 holds for the other endcap

$$
A_{L}^{(u)} \equiv \sum_{n=1}^{\infty} A_{L}^{(n, u)}=A^{(u)}+\left[i \mathcal{K}_{2} i F+i \mathcal{K}_{3, L}^{(u, u)} \frac{i F}{2 \omega L^{3}} 2 A^{(s)}\right],
$$

while for the correlator sum we obtain

$$
\sum_{n=0}^{\infty} C_{L, 0 F}^{(n)}=C_{\infty}^{\left[B_{2}\right]}+2 A^{\prime(s)} \frac{i F}{2 \omega L^{3}} A_{L}^{(u)}
$$

where

$$
C_{\infty}^{\left[B_{2}\right]}=\sum_{n=0}^{\infty} C_{\infty}^{(n)}
$$

Substituting Eq. (5.192) into the result (5.193) allows us to rewrite the latter in terms of a single finite-volume quantity, $\mathcal{K}_{3, L}^{(u, u)}$.

We can now express $C_{L}^{\left[B_{2}\right]}$ in terms of infinite-volume quantities together with $\mathcal{K}_{3, L}^{(u, u)}$. This requires substituting Eqs. (5.190), (5.192) and (5.193) into Eq. (5.178) and using the following identities

$$
\begin{gathered}
\left(i F i \mathcal{K}_{2}+\frac{i F}{2 \omega L^{3}} i \mathcal{K}_{3, L}^{(u, u)}\right)[\mathcal{A}] \sum_{n=0}^{\infty}\left(i \mathcal{K}_{3, L}^{(u, u)}[\mathcal{A}]\right)^{n}=[\mathcal{A}] \sum_{n=0}^{\infty}\left(i \mathcal{K}_{3, L}^{(u, u)}[\mathcal{A}]\right)^{n}-\frac{i F}{2 \omega L^{3}} \\
=[\mathcal{A}] \sum_{n=0}^{\infty}\left(i \mathcal{K}_{3, L}^{(u, u)}[\mathcal{A}]\right)^{n}\left(i \mathcal{K}_{2} i F+i \mathcal{K}_{3, L}^{(u, u)} \frac{i F}{2 \omega L^{3}}\right)
\end{gathered}
$$

After some algebra, we obtain a relatively simple form

$$
C_{L}^{\left[B_{2}\right]}=C_{\infty}^{\left[B_{2}\right]}+\delta C_{\infty}^{\left[B_{2}\right]}+A^{\prime\left[B_{2}\right]}\left[-\frac{2}{3} \frac{i F}{2 \omega L^{3}}+[\mathcal{A}] \sum_{n=0}^{\infty}\left(i \mathcal{K}_{3, L}^{(u, u)}[\mathcal{A}]\right)^{n}\right] A^{\left[B_{2}\right]},
$$


where

$$
A^{\prime\left[B_{2}\right]}=\sigma^{*}+A^{\prime(u)}+A^{\prime(s)}+A^{\prime(\widetilde{s})}, \quad A^{\prime\left[B_{2}\right]}=\sigma^{\dagger *}+A^{(u)}+A^{(s)}+A^{(\widetilde{s})},
$$

and

$$
\delta C_{\infty}^{\left[B_{2}\right]}=\frac{2}{3} A^{\prime\left[B_{2}\right]} \frac{i F}{2 \omega L^{3}}\left(A^{(u)}-A^{(s)}\right)+\frac{2}{3}\left(A^{\prime(u)}-A^{\prime(s)}\right) \frac{i F}{2 \omega L^{3}} \sigma^{\dagger *} .
$$

Several comments are in order. First, we observe that summing over all switches has led to a dramatic simplification in the expression for the correlator. This can be seen, for example, by comparing even the one-switch expression (5.153) to Eq. (5.197). Second, to obtain Eq. (5.197) we have made use of the fact, explained after Eq. (5.149), that, within our derivation thus far, superscripts $(s)$ and $(\widetilde{s})$ are interchangeable. This allows us to write the result in terms of endcaps, $A^{\prime\left[B_{2}\right]}$ and $A^{\left[B_{2}\right]}$, which are symmetric under particle interchange. We stress that this symmetrization occurs only when working to all orders in the number of switches, since it requires combining terms with different numbers of switches. Our third comment also concerns symmetrization, or rather the its absence in Eq. (5.199). Recall that particle-interchange symmetry was violated when we chose to analyze the loops in $C_{L, 0 F}^{(n)}$ moving from left to right, since this led to $(s)$ quantities always being to the left of those with superscripts $(u)$. Forcing the endcaps into symmetric form leads to the remainder $\delta C_{\infty}^{\left[B_{2}\right]}$. Note that in the terms involving a $(u)-(s)$ difference, we can freely interchange $(s)$ and $(\widetilde{s})$, and we have used this freedom to choose both terms to involve $(s)$. Although $\delta C_{\infty}$ appears to be a finite-volume term (since it contains factors of $F$ ), in fact, as we show below, it can be rewritten as an infinite-volume quantity. This means that $\delta C_{\infty}^{\left[B_{2}\right]}$ can be absorbed into an alternative infinite-volume quantity, used in place of $C_{\infty}^{\left[B_{2}\right]}$. Since other contributions of this type arise in the analysis that follows, we delay our definition of the replacement until Eq. (5.243) below. We note that our job is not done, because the result (5.197) still contains the asymmetric three-particle finite-volume scattering amplitude $\mathcal{K}_{3, L}^{(u, u)}$. We return shortly to the task of rewriting this in terms of infinite-volume quantities.

First, however, we rewrite $\delta C_{\infty}^{\left[B_{2}\right]}$ in a manifestly infinite-volume form. We show how this works for the first term in 5.199 from which the generalization to the second term is 
immediate. The steps are as follows:

$$
\begin{aligned}
& \frac{2}{3} A^{\prime\left[B_{2}\right]} \frac{i F}{2 \omega L^{3}}\left(A^{(u)}-A^{(s)}\right)=\frac{1}{3} \frac{1}{L^{3}} \sum_{\vec{k}}\left[\frac{1}{L^{3}} \sum_{\vec{a}}-\widetilde{\mathrm{PV}} \int_{\vec{a}}\right] \\
& \times A^{\prime\left[B_{2}\right]}(\vec{k}, \vec{a}) \frac{i H(\vec{k}) H(\vec{a}) H\left(\vec{b}_{k a}\right)}{2 \omega_{k} 2 \omega_{a} 2 \omega_{k a}\left(E-\omega_{k}-\omega_{a}-\omega_{k a}\right)}\left[A^{(u)}(\vec{k}, \vec{a})-A^{(u)}(\vec{a}, \vec{k})\right] \\
& =-\frac{1}{3} \int_{\vec{k}} \widetilde{\mathrm{PV}} \int_{\vec{a}} A^{\prime\left[B_{2}\right]}(\vec{k}, \vec{a}) \frac{i H(\vec{k}) H(\vec{a}) H\left(\vec{b}_{k a}\right)}{2 \omega_{k} 2 \omega_{a} 2 \omega_{k a}\left(E-\omega_{k}-\omega_{a}-\omega_{k a}\right)}\left[A^{(u)}(\vec{k}, \vec{a})-A^{(u)}(\vec{a}, \vec{k})\right] \\
& =\int_{\vec{k}} A^{\prime\left[B_{2}\right]}(\vec{k}) \frac{i \rho(\vec{k})}{3 \omega_{k}}\left[A^{(u)}(\vec{k})-A^{(s)}(\vec{k})\right]=A^{\prime\left[B_{2}\right]} \frac{i \rho}{3 \omega}\left[A^{(u)}-A^{(s)}\right],
\end{aligned}
$$

where in an abuse of notation, in the last line we have introduced the shorthand that integration over $\vec{k}$ is implicit for a product involving $\rho$.

In the first step, we use the sum-minus-integral identity in reverse, as well as the definition of $A^{(s)}$ [see Eq. 5.141)]. The momenta $\vec{k}$ and $\vec{a}$ are on shell, but, in general, the third four-momentum, $b_{k a}=P-k-a$, is now off shell, Thus amplitudes are not invariant under the full particle-interchange symmetry. Nevertheless, $A^{\prime\left[B_{2}\right]}(\vec{k}, \vec{a})$ remains symmetric under the interchange $\vec{k} \leftrightarrow \vec{a}$, while the $A^{(u)}-A^{(s)}$ becomes a term which is manifestly antisymmetric under this interchange. Since the remaining terms are symmetric, the entire summand/integrand is antisymmetric. This observation allows us to drop the double sum, since a symmetric sum over an antisymmetric summand clearly vanishes. The sum over $\vec{k}$ can now be replaced by an integral, since the $\widetilde{\mathrm{PV}}$ integral over $\vec{a}$ leads to a smooth function of $\vec{k}$. At this stage we obtain the second form of the right-hand side. The final step is to notice that, if an $i \epsilon$ pole prescription were used, then the double integration would also vanish by symmetry. Thus it is only the $\rho$ term in the definition of $\widetilde{\mathrm{PV}}$ integration that survives, and its form is obtained using Eqs. (5.59) and (5.60).

Applying a similar analysis to second term in Eq. (5.199), we find, in total,

$$
\delta C_{\infty}^{\left[B_{2}\right]}=A^{\prime\left[B_{2}\right]} \frac{i \rho}{3 \omega}\left[A^{(u)}-A^{(s)}\right]+\left[A^{\prime(u)}-A^{\prime(s)}\right] \frac{i \rho}{3 \omega} \sigma^{\dagger *}
$$

where, as above, integration over $\vec{k}$ is implicit.

This is a manifestly infinite-volume quantity depending only on on-shell (but not symmetric) amplitudes. 
(a)

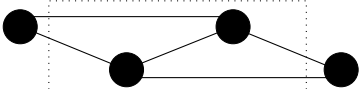

(b)

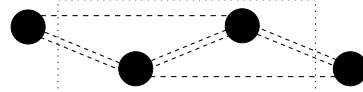

(c)

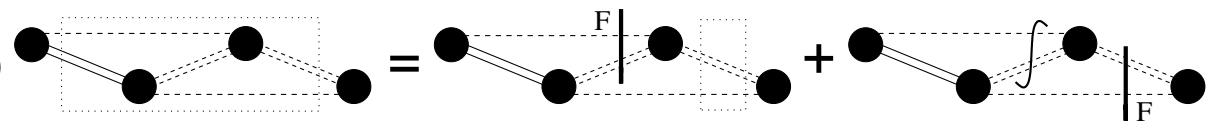
$+0 \int_{1}^{0}$

(d)
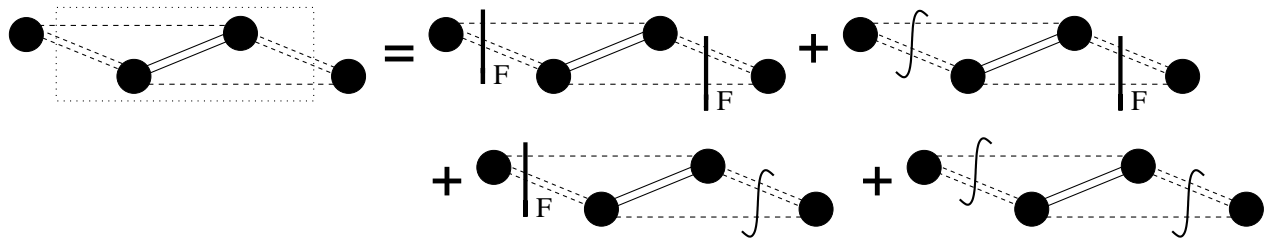

(e)

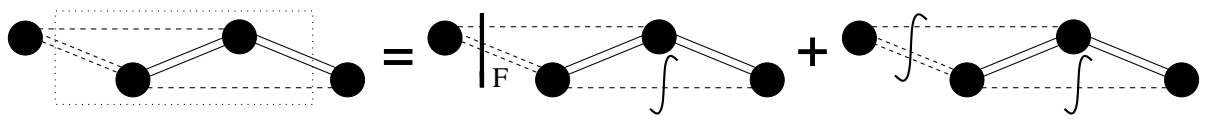

(f)

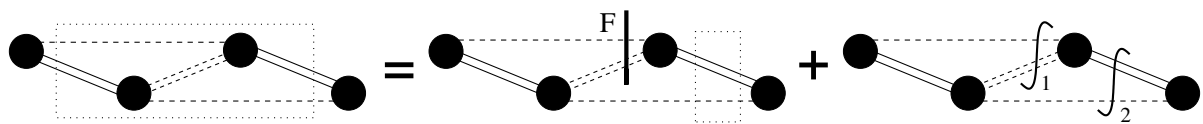

(g)

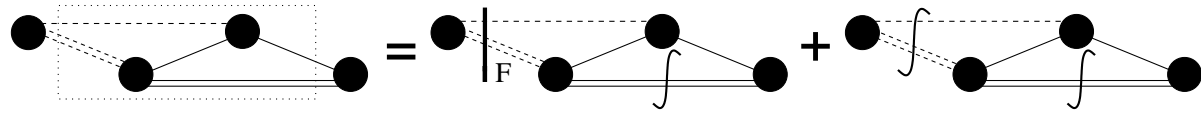

(h)

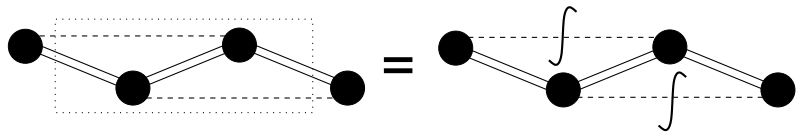

(i)

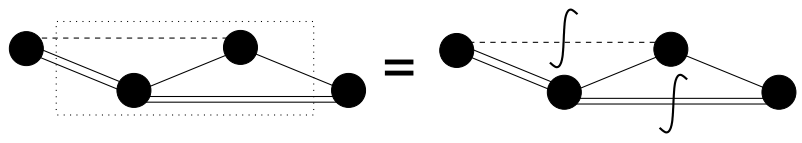

(j)

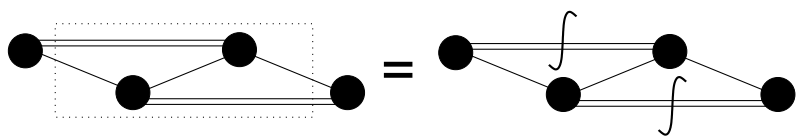

Figure 5.18: Decomposition of $\mathcal{K}_{3, L}^{(4, u, u)}$. All external propagators are dropped, and the notation of Figs. 5.12 and 5.16 is used. (a) $\mathcal{K}_{3, L}^{(4, u, u)}$ itself [see Eq. 5.175]]; (b) the most singular term (with three singular propagators); (c) and (d): terms with two singular propagators and their decompositions; (e), (f) and (g): terms with one singular propagator and their decompositions; (h), (i) and (j): non-singular terms. Terms in the decompositions are always ordered from most to least singular. The treatment of loop momenta is indicated explicitly: they are either summed (dashed box), integrated (integral sign) or the sum-minus-integral identity is used (factor of $F$ ). Where the order of integrals matters it is shown explicitly. 
The final identity we require is that for $\mathcal{K}_{3, L}^{(n, u, u)}$. The identities for $n=2$ and 3 are given, respectively, by Eqs. (5.138) and (5.172). To understand how the pattern generalizes to arbitrary $n$ it is useful to first work out explicitly the result for $n=4$, since new effects occur at this order. The decomposition of $\mathcal{K}_{3, L}^{(4, u, u)}$ is shown diagrammatically in Fig. 5.18 . Here we are using a stripped-down diagrammatic notation in which external lines and momentum labels are implicit. The basic method, however, is exactly as used earlier for $n=2$ and 3: (i) do the time-component integrals over the loop momenta, and separate the result into on-shell particle contributions and the remainders; (ii) separate each of the remaining "diagonal" propagators and their attached factors of $\mathcal{K}_{2}$ into a singular part (containing $G^{b}$ ) and the non-singular remainder; (iii) pull out the most singular term; (iv) analyze the remainder by converting sums into integrals where possible, which in some cases leads to residues containing factors of $F$. The key point is that after the most singular term has been subtracted, there is always at least one choice of ordering of momentum sums in which allows the use of the sum-minus-integral identity at each stage. For most terms in the decomposition there is either a single such choice or the order is unimportant. However, at $n=4$ we first encounter a case where there is a significant choice of ordering to be made. As $n$ increases there are more such cases and we need a convention for how to deal with them.

We now work through the different contributions to $\mathcal{K}_{3, L}^{(4, u, u)}$ in some detail, starting from the most singular and working to the least. We recall the notation [from Eq. 5.175) and Fig. 5.17] that $q_{1}$ is the leftmost loop momentum and $q_{2}$ the rightmost. The most singular term is that shown in Fig. 5.18 b, and gives the contribution

$$
\text { (b) }=i \mathcal{K}_{2} i G i \mathcal{K}_{2} i G i \mathcal{K}_{2} i G\left[2 \omega L^{3}\right] i \mathcal{K}_{2}
$$

This term must be left as a sum (which is implicit in our matrix notation).

Contributions with two singular propagators are those of Fig. 5.18c, its "reflection" (not shown) in which the rightmost diagonal propagator is non-singular, and Fig. 5.18d. The decomposition of the first of these is also shown in Fig. 5.18c. We must begin with $q_{1}$ since the $q_{2}$ sum runs over two singular propagators. We first convert the $q_{1}$ sum into an $F$-insertion plus an integral. For the $F$-term this is as far as we can go, since the $q_{2}$ sum 
runs over singularities in both $F$ and the propagator. For the integral over $q_{1}$ we can repeat the $F$ plus integral decomposition for $q_{2}$. Note that in the resulting double integral the order of integration is important. The net result is that there are three terms, each with different levels of singularity. The doubly-singular term gives

$$
\text { (c) [doubly singular }]=2 i \mathcal{K}_{\mathrm{df}, 3}^{(2, u, s)} \frac{i F}{2 \omega L^{3}} i \mathcal{K}_{2} i G\left[2 \omega L^{3}\right] i \mathcal{K}_{2},
$$

while the term with one singularity is a contribution to

$$
\text { (c) [singly singular }] \subset 2 i \mathcal{K}_{\mathrm{df}, 3}^{(3, u, s)} i F i \mathcal{K}_{2},
$$

and the non-singular term contributes to

$$
\text { (c) [non-singular }] \subset i \mathcal{K}_{\mathrm{df}, 3}^{(4, u, u)},
$$

The reflected contribution is decomposed similarly.

The decomposition of the remaining term with two singular propagators is shown in Fig. 5.18d. Here, since the singular propagators are separated, the sum-integral identity can be applied to each independently. Thus there are four terms in the decomposition. The doubly-singular one is

$$
\text { (d) [doubly singular }]=i \mathcal{K}_{2} i F 4 i \mathcal{K}_{\mathrm{df}, 3}^{(2, s, s)} i F i \mathcal{K}_{2} \text {. }
$$

Note that here both $(u)$ 's have been switched to $(s)$ 's. Each switch comes with a factor of 2 , leading to the overall factor of 4 . The singly-singular terms contribute to $2 i \mathcal{K}_{\mathrm{df}, 3}^{(3, u, s)} i F i \mathcal{K}_{2}$ and $i \mathcal{K}_{2} i F 2 i \mathcal{K}_{\mathrm{df}, 3}^{(3, s, u)}$, while the non-singular terms contribute to $i \mathcal{K}_{\mathrm{df}, 3}^{(4, u, u)}$.

There are three diagrams containing one singular propagator: Fig. 5.18, its reflection, and Fig. 5.18f. In the first, the sum over $q_{2}$ can be immediately converted to an integral, since the summand is non-singular. For $q_{1}$ we obtain the usual $F$-term plus integral. The former gives rise to another contribution to $i \mathcal{K}_{2} i F 2 i \mathcal{K}_{\mathrm{df}, 3}^{(3, s, u)}$, while the latter contributes to $i \mathcal{K}_{\mathrm{df}, 3}^{(4, u, u)}$. Analogous results hold for the reflecton of Fig. 5.18 .

The diagram of Fig. 5.18 leads to a new effect. Here we can use the sum-integral identity either on $\vec{q}_{1}$ or $\vec{q}_{2}$. Our convention (as above) is to work from left to right when there is such a choice. This gives the singly-singular term

$$
\text { (f) [singly singular }]=2 i \mathcal{K}_{\mathrm{df}, 3}^{(2, u, s)} \frac{i F}{2 \omega L^{3}} i \mathcal{K}_{\mathrm{df}, 3}^{(2, u, u)}
$$


where our convention has led to the $(s)$ being on the left side of the $F$, rather than on the right. The non-singular term contributes to $i \mathcal{K}_{\mathrm{df}, 3}^{(4, u, u)}$. Here our convention leads to a definite (left to right) ordering of the $\widetilde{\mathrm{PV}}$ integrals.

Another new feature of the $n=4$ analysis is the appearance of singular contributions in which one of the $q_{j}^{0}$ integrals does not circle the particle pole. The corresponding diagrams are Fig. 5.18g and its reflection. The decomposition exactly follows that of Fig. 5.18.

Finally, we reach the completely non-singular contributions, where sums can be immediately converted to integrals. There are four such diagrams, Fig. 5.18h, its reflection, Fig. 5.18 and Fig. 5.18j. These all contribute to $i \mathcal{K}_{\mathrm{df}, 3}^{(4, u, u)}$.

Adding all contributions we find the total result

$$
\begin{array}{r}
i \mathcal{K}_{3, L}^{(4, u, u)}=i \mathcal{K}_{2} i G i \mathcal{K}_{2} i G i \mathcal{K}_{2}\left[i G 2 \omega L^{3}\right] i \mathcal{K}_{2}+i \mathcal{K}_{2} i G\left[2 \omega L^{3}\right] i \mathcal{K}_{2} \frac{i F}{2 \omega L^{3}} 2 i \mathcal{K}_{\mathrm{df}, 3}^{(2, s, u)} \\
+2 i \mathcal{K}_{\mathrm{df}, 3}^{(2, u, s)} \frac{i F}{2 \omega L^{3}} i \mathcal{K}_{2} i G\left[2 \omega L^{3}\right] i \mathcal{K}_{2}+i \mathcal{K}_{2} i F 4 i \mathcal{K}_{\mathrm{df}, 3}^{(2, s, s)} i F i \mathcal{K}_{2}+2 i \mathcal{K}_{\mathrm{df}, 3}^{(3, u, s)} i F i \mathcal{K}_{2} \\
+i \mathcal{K}_{2} i F 2 i \mathcal{K}_{\mathrm{df}, 3}^{(3, s, u)}+2 i \mathcal{K}_{\mathrm{df}, 3}^{(2, u, s)} \frac{i F}{2 \omega L^{3}} i \mathcal{K}_{\mathrm{df}, 3}^{(2, u, u)}+i \mathcal{K}_{\mathrm{df}, 3}^{(4, u, u)}
\end{array}
$$

where we have ordered terms in decreasing strength of divergence. The only aspect of this result not explained above is that contributions combine properly to give the quantities $\mathcal{K}_{\mathrm{df}, 3}^{(3, u, s)}$ and $\mathcal{K}_{\mathrm{df}, 3}^{(3, s, u)}$ in the fifth and sixth terms, respectively. For example, the $\mathcal{K}_{\mathrm{df}, 3}^{(3, u, s)}$ term receives the required four contributions (see Fig. 5.16) from diagrams (c), (d) and the reflections of (e) and (g). One can demonstrate that the correct contributions occur in all cases by observing that (i) the result 5.210 provides a complete classification of possible divergence structures and (ii) that expanding out each term in 5.210 leads to a unique set of contributions each of which is necessarily present in the decomposition of $\mathcal{K}_{3, L}^{(4, u, u)}$. Finally, we note that the non-singular term in Eq. $5.210, \mathcal{K}_{\mathrm{df}, 3}^{(4, u, u)}$, is simply defined as the sum of contributions from all the diagrams in Fig. 5.18 (plus appropriate reflections) that contain only loop integrals.

We are now ready to explain the result for general $i \mathcal{K}_{3, L}^{(n, u, u)}$. What arises are sequences alternating between one of the $\mathcal{K}$ 's,

$$
i \mathcal{K}_{2}, i \mathcal{K}_{\mathrm{df}, 3}^{(j, u, u)}, 2 i \mathcal{K}_{\mathrm{df}, 3}^{(j, s, u)}, 2 i \mathcal{K}_{\mathrm{df}, 3}^{(j, u, s)} \text { and } 4 i \mathcal{K}_{\mathrm{df}, 3}^{(j, s, s)}
$$


and one of

$$
\frac{i F}{2 \omega L^{3}} \text { and } i G \text {. }
$$

All possible combinations should be included, subject to the following rules

- The number of switches must add up to $n$. This number is given by the total number of $F$ 's and $G$ 's plus the number of switches in the $\mathcal{K}_{\mathrm{df}, 3}$ 's.

- There must be a $\mathcal{K}_{2}$ or $\mathcal{K}_{\mathrm{df}, 3}$ on both ends.

- Each $\mathcal{K}_{\mathrm{df}, 3}$ must have $F$ on both sides unless external. This is because the loop momenta next to a $\mathcal{K}_{\mathrm{df}, 3}$ have only one singular propagator in their summands and so the sum-integral identity can be used. This implies, given the rules above, that each $G$ must have a $\mathcal{K}_{2}$ (and not a $\left.\mathcal{K}_{\mathrm{df}, 3}\right)$ on both sides.

- F's must have a $\mathcal{K}_{\mathrm{df}, 3}$ on at least one side, or, equivalently, $F$ 's always appear on one side or other of a $\mathcal{K}_{\mathrm{df}, 3}$. This is because one cannot use the sum-integral identity in the middle of a sequence of singular propagators, since each loop sum runs over two singularities. The identity can only be used at the end of the sequence, and only then if it terminates with the non-singular part of a propagator. An example of this rule is that Fig. 5.18b cannot be decomposed using the sum-integral identity, whereas Fig. 5.18c can at the left-hand end. A consequence of this rule is that the only long subsequences involving $\mathcal{K}_{2}$ have the form $\ldots i \mathcal{K}_{2} i G i \mathcal{K}_{2} i G i \mathcal{K}_{2} \ldots$ These correspond to diagrams with sequences of singular propagators.

- In a sequence of the form $\ldots i \mathcal{K}_{2} i G i \mathcal{K}_{2} i G i \mathcal{K}_{2} \ldots$ the rightmost $G$ is multiplied on the right by $\left[2 \omega L^{3}\right]$. This arises from keeping track of on-shell propagators.

- The right-hand superscript of each $\mathcal{K}_{\mathrm{df}, 3}$ is $(s)$ unless it is external, when it is a $(u)$. Examples are the third, fifth and seventh terms in the expression 5.210 for $\mathcal{K}_{3, L}^{(4, u, u)}$.

- The middle superscript of each $\mathcal{K}_{\mathrm{df}, 3}$ is $(s)$ unless it is either external or it appears to the right of another $\mathcal{K}_{\mathrm{df}, 3}$, separated by a single $F$, in which cases it is a $(u)$. The 
difference from the previous rule arises due to our "left-to-right" convention of dealing with loop momenta. An example of the new exception is given by the penultimate term in Eq. (5.210).

A simple consequence of these rules is that the most divergent contribution to $i \mathcal{K}_{3, L}^{(n, u, u)}$ is

$$
i \mathcal{K}_{2}\left(i G i \mathcal{K}_{2}\right)^{n-2} i G\left[2 \omega L^{3}\right] i \mathcal{K}_{2}
$$

Similarly, sequences having this form (but with smaller values of $n$ ) can appear both connecting the ends to factors of $\mathcal{K}_{\mathrm{df}, 3}$, or between such factors.

It is simpler to display the full result for the summed quantity $\mathcal{K}_{3, L}^{(u, u)}=\sum_{n=2}^{\infty} \mathcal{K}_{3, L}^{(n, u, u)}$ than for $\mathcal{K}_{3, L}^{(n, u, u)}$. This removes the constraint of the first rule, so that the sequences are now composed of the quantities

$$
\mathcal{K}_{\mathrm{df}, 3}^{(u, u)} \equiv \sum_{n=2}^{\infty} \mathcal{K}_{\mathrm{df}, 3}^{(n, u, u)}, \quad \mathcal{K}_{\mathrm{df}, 3}^{(u, s)} \equiv \sum_{n=2}^{\infty} \mathcal{K}_{\mathrm{df}, 3}^{(n, u, s)}, \quad \mathcal{K}_{\mathrm{df}, 3}^{(s, u)} \equiv \sum_{n=2}^{\infty} \mathcal{K}_{\mathrm{df}, 3}^{(n, s, u)} \text { and } \mathcal{K}_{\mathrm{df}, 3}^{(s, s)} \equiv \sum_{n=2}^{\infty} \mathcal{K}_{\mathrm{df}, 3}^{(n, s, s)}
$$

In addition, the sequences of divergent terms of the form 5.213 can be summed, leading to

$$
\sum_{n=2}^{\infty} i \mathcal{K}_{2}\left(i G i \mathcal{K}_{2}\right)^{n-2} i G\left[2 \omega L^{3}\right] i \mathcal{K}_{2}=i T i G\left[2 \omega L^{3}\right] i \mathcal{K}_{2}=i \mathcal{K}_{2} i G i T\left[2 \omega L^{3}\right],
$$

where

$$
i T \equiv \frac{1}{1-i \mathcal{K}_{2} i G} i \mathcal{K}_{2}=i \mathcal{K}_{2} \frac{1}{1-i G i \mathcal{K}_{2}}
$$

We have used here the result that $\mathcal{K}_{2}$ commutes with $\left[2 \omega L^{3}\right]$, since both are diagonal.

To show the result in a compact form we collect the $\mathcal{K}_{\mathrm{df}, 3}$ into a two-by-two matrix. Now is a good point to recall that, using the arguments following Eq. (5.149), we can freely interchange in our formulae the superscripts $(s)$ and $(\widetilde{s})$. This is allowed because the rules always lead to quantities with $(s)$ superscripts being adjacent to those with $(u)$ superscripts (with an intervening factor of $F$ ). This allows us, for example, to replace $2 \mathcal{K}_{\mathrm{df}, 3}^{(s, u)}$ with $\mathcal{K}_{\mathrm{df}, 3}^{(s, u)}+\mathcal{K}_{\mathrm{df}, 3}^{(\widetilde{s}, u)}$. The point of such changes is to move towards a physical quantity which contains the symmetric combination $(u)+(s)+(\widetilde{s})$ for all superscripts. With this in mind, 
we introduce the matrix of matrices

$$
\left(i \mathcal{K}_{\mathrm{df}, 3}\right) \equiv\left(\begin{array}{cc}
i \mathcal{K}_{\mathrm{df}, 3}^{(u, u)} & i \mathcal{K}_{\mathrm{df}, 3}^{(u, s)}+i \mathcal{K}_{\mathrm{df}, 3}^{(u, \widetilde{s})} \\
i \mathcal{K}_{\mathrm{df}, 3}^{(s, u)}+i \mathcal{K}_{\mathrm{df}, 3}^{(\widetilde{s}, u)} & i \mathcal{K}_{\mathrm{df}, 3}^{(s, s)}+i \mathcal{K}_{\mathrm{df}, 3}^{(s, \widetilde{s})}+i \mathcal{K}_{\mathrm{df}, 3}^{(\widetilde{s}, s)}+i \mathcal{K}_{\mathrm{df}, 3}^{(\widetilde{s}, \widetilde{s})}
\end{array}\right)
$$

The quantity symmetric under particle exchange is then

$$
i \mathcal{K}_{\mathrm{df}, 3}^{\left[B_{2}\right]} \equiv\left(\begin{array}{cc}
1 & 1
\end{array}\right)\left(i \mathcal{K}_{\mathrm{df}, 3}\right)\left(\begin{array}{c}
1 \\
1
\end{array}\right)
$$

Using this matrix notation, and implementing the rules described above, we find

$$
\begin{aligned}
i \mathcal{K}_{3, L}^{(u, u)}= & i T i G\left[2 \omega L^{3}\right] i \mathcal{K}_{2}+\left(\begin{array}{cc}
1 & i T i F
\end{array}\right)\left(i \mathcal{K}_{\mathrm{df}, 3}\right) \\
& \times \sum_{j=0}^{\infty}\left\{\left(\begin{array}{c}
0 \\
1
\end{array}\right) \frac{i F}{2 \omega L^{3}}\left(\begin{array}{cc}
1 & i T i F
\end{array}\right)\left(i \mathcal{K}_{\mathrm{df}, 3}\right)\right\}^{j}\left(\begin{array}{c}
1 \\
\frac{i F}{2 \omega L^{3}} i T 2 \omega L^{3}
\end{array}\right) .
\end{aligned}
$$

We have succeeded in pulling out explicit finite-volume factors, with the infinite-volume quantities being $\mathcal{K}_{2}$ and the two-by-two matrix, $\left(\mathcal{K}_{\mathrm{df}, 3}\right)$. The latter, however, does not appear in the symmetric form (5.218). In particular, our left-to-right convention leads to the presence of an assymetric matrix between factors of $\left(\mathcal{K}_{\mathrm{df}, 3}\right)$.

The final step is to insert the result 5.219 into our expression for $C_{L}^{\left[B_{2}\right]}$, Eq. 5.197, and simplify. We begin by keeping only the first term in 5.219 , i.e. that which arises from summing the most divergent contributions to $\mathcal{K}_{3, L}^{(u, u)}$. We find

$$
\begin{aligned}
C_{L}^{\left[B_{2}\right]}-C_{\infty}^{\left[B_{2}\right]}-\delta C_{\infty}^{\left[B_{2}\right]} & =A^{\prime\left[B_{2}\right]}\left[-\frac{2}{3} \frac{i F}{2 \omega L^{3}}+[\mathcal{A}] \sum_{n=0}^{\infty}\left(i \mathcal{K}_{3, L}^{(u, u)}[\mathcal{A}]\right)^{n}\right] A^{\left[B_{2}\right]} \\
& =A^{\prime\left[B_{2}\right]} i F_{3} A^{\left[B_{2}\right]}+\mathcal{O}\left(\mathcal{K}_{\mathrm{df}, 3}\right)
\end{aligned}
$$

where the first line is a restatement of Eq. (5.197), and the second contains the new quantity

$$
i F_{3}=\frac{i F}{2 \omega L^{3}}\left[-\frac{2}{3}+\frac{1}{1-i T i F}\right] \text {. }
$$


To obtain this form for $F_{3}$ we have used

$$
[\mathcal{A}] \sum_{n=0}^{\infty}\left(i T i G\left[2 \omega L^{3}\right] i \mathcal{K}_{2}[\mathcal{A}]\right)^{n}=\frac{i F}{2 \omega L^{3}} \frac{1}{1-i T i F}
$$

Here the two sides are different ways of writing the sum of sequences in which $F$ 's or $G$ 's alternate with $\mathcal{K}_{2}$ 's in all possible orders, with the constraint that sequences must have $F$ 's at both ends. On the left one sums first over the number of intermediate $F$ 's and then sums over $G$ 's, while on the right the roles of $F$ and $G$ are interchanged. In the following we will also need two further ways of writing this quantity

$$
\frac{i F}{2 \omega L^{3}} \frac{1}{1-i T i F}=\left\{\sum_{n=0}^{\infty}\left([\mathcal{A}] i \operatorname{Ti} G\left[2 \omega L^{3}\right] i \mathcal{K}_{2}\right)^{n}\right\}[\mathcal{A}]=\frac{1}{1-\frac{i F}{2 \omega L^{3}} i T\left[2 \omega L^{3}\right]} \frac{i F}{2 \omega L^{3}}
$$

Next we consider terms proportional to $\mathcal{K}_{\mathrm{df}, 3}$. These are obtained by replacing one of the factors of $\mathcal{K}_{3, L}^{(u, u)}$ in the sum over $n$ in Eq. 5.220 with the term linear in $\mathcal{K}_{\mathrm{df}, 3}$ from Eq. 5.219, with all other $\mathcal{K}_{3, L}^{(u, u)}$, s replaced by the most divergent term from Eq. 5.219. This leads to the contribution

$$
\begin{aligned}
C_{L}^{\left[B_{2}\right]}-C_{\infty}^{\left[B_{2}\right]}-\delta C_{\infty}^{\left[B_{2}\right]} \supset & \\
A^{\prime\left[B_{2}\right]} \frac{i F}{2 \omega L^{3}} \frac{1}{1-i T i F}\left(\begin{array}{cc}
1 & i T i F
\end{array}\right) & \left(i \mathcal{K}_{\mathrm{df}, 3}\right)\left(\begin{array}{c}
1 \\
\frac{i F}{2 \omega L^{3}} i T 2 \omega L^{3}
\end{array}\right) \\
& \times \frac{1}{1-\frac{i F}{2 \omega L^{3}} i T\left[2 \omega L^{3}\right]} \frac{i F}{2 \omega L^{3}} A^{\left[B_{2}\right]} .
\end{aligned}
$$

We next use the identities

$$
\begin{gathered}
\frac{i F}{2 \omega L^{3}} \frac{1}{1-i T i F}\left(\begin{array}{cc}
1 & i T i F
\end{array}\right)=i F_{3}\left(\begin{array}{cc}
1 & 1
\end{array}\right)+\frac{i F}{2 \omega L^{3}}\left(\begin{array}{cc}
\frac{2}{3} & -\frac{1}{3}
\end{array}\right) \\
\left(\begin{array}{c}
1 \\
\frac{i F}{2 \omega L^{3}} i T 2 \omega L^{3}
\end{array}\right) \frac{1}{1-\frac{i F}{2 \omega L^{3}} i T\left[2 \omega L^{3}\right]} \frac{i F}{2 \omega L^{3}}=\left(\begin{array}{c}
1 \\
1
\end{array}\right) i F_{3}+\left(\begin{array}{c}
2 / 3 \\
-1 / 3
\end{array}\right) \frac{i F}{2 \omega L^{3}}
\end{gathered}
$$


to rewrite the right-hand side of Eq. (5.225) as

$$
\begin{aligned}
A^{\prime\left[B_{2}\right]}\left\{i F_{3}\left(\begin{array}{ll}
1 & 1
\end{array}\right)+\frac{i F}{2 \omega L^{3}}\left(\begin{array}{cc}
\frac{2}{3} & -\frac{1}{3}
\end{array}\right)\right\}\left(i \mathcal{K}_{\mathrm{df}, 3}\right) \\
\times\left\{\left(\begin{array}{c}
1 \\
1
\end{array}\right) i F_{3}+\left(\begin{array}{c}
2 / 3 \\
-1 / 3
\end{array}\right) \frac{i F}{2 \omega L^{3}}\right\} A^{\left[B_{2}\right]} .
\end{aligned}
$$

Here we have separated out the symmetric part of $\left(\mathcal{K}_{\mathrm{df}, 3}\right)$, which is multiplied on both sides by $F_{3}$, from the asymmetric parts. The latter can be analyzed in the same way as $\delta C_{\infty}^{\left[B_{2}\right]}$ [see Eq. 5.202 and subsequent text]. This is because the vector $(2,-1)$ projects, both from the left and right, onto a $(u)-(s)$ combination (if we use the freedom to interchange $(\widetilde{s})$ and $(s)$ when separated from symmetric quantity such as $A^{\prime[B 2]}$ by an $F$ ). For example, using this freedom one finds

$$
\begin{aligned}
& A^{\prime\left[B_{2}\right]} \frac{i F}{2 \omega L^{3}}\left(\begin{array}{cc}
\frac{2}{3} & -\frac{1}{3}
\end{array}\right)\left(i \mathcal{K}_{\mathrm{df}, 3}\right)\left(\begin{array}{c}
1 \\
1
\end{array}\right)= \\
& A^{\prime\left[B_{2}\right]} \frac{i F}{2 \omega L^{3}} \frac{2}{3}\left\{i \mathcal{K}_{\mathrm{df}, 3}^{(u, u)}-i \mathcal{K}_{\mathrm{df}, 3}^{(s, u)}+i \mathcal{K}_{\mathrm{df}, 3}^{(u, s)}-i \mathcal{K}_{\mathrm{df}, 3}^{(s, s)}+i \mathcal{K}_{\mathrm{df}, 3}^{(u, \widetilde{s})}-i \mathcal{K}_{\mathrm{df}, 3}^{(s, \widetilde{s})}\right\}
\end{aligned}
$$

This means that, just as in Eq. 5.202, $F / L^{3}$ can be replaced by $\rho$ with the (implicit) sum over the spectator momentum replaced by an integral. The same holds for the $F$ on the right of $\left(\mathcal{K}_{\mathrm{df}, 3}\right)$. We can therefore rewrite Eq. (5.228) as

$$
A^{\prime\left[B_{2}\right]}\left\{i F_{3}\left(\begin{array}{ll}
1 & 1
\end{array}\right)+\frac{i \rho}{2 \omega}\left(\begin{array}{cc}
\frac{2}{3} & -\frac{1}{3}
\end{array}\right)\right\}\left(i \mathcal{K}_{\mathrm{df}, 3}\right)\left\{\left(\begin{array}{c}
1 \\
1
\end{array}\right) i F_{3}+\left(\begin{array}{c}
2 / 3 \\
-1 / 3
\end{array}\right) \frac{i \rho}{2 \omega}\right\} A^{\left[B_{2}\right]}
$$

again using the notation with implicit integration for $\rho$ factors that was introduced in Eq. (5.202). The contribution linear in $\mathcal{K}_{\mathrm{df}, 3}$ can thus be broken up into four parts: (i) a finite-volume term involving symmetric quantities

$$
A^{\prime\left[B_{2}\right]} i F_{3} i \mathcal{K}_{\mathrm{df}, 3}^{\left[B_{2}\right]} i F_{3} A^{\left[B_{2}\right]}
$$


(ii) a partially asymmetric term with $\rho$ on the left

$$
A^{\prime\left[B_{2}\right]} i F_{3} \frac{i \rho}{2 \omega}\left(\begin{array}{cc}
\frac{2}{3} & -\frac{1}{3}
\end{array}\right)\left(i \mathcal{K}_{\mathrm{df}, 3}\right)\left(\begin{array}{c}
1 \\
1
\end{array}\right) i F_{3} A^{\left[B_{2}\right]},
$$

which can be interpreted as $\delta\left(A^{\prime\left[B_{2}\right]}\right) i F_{3} A^{\left[B_{2}\right]}$, where $\delta\left(A^{\prime\left[B_{2}\right]}\right)$ absorbs the infinite-volume integral involving $\rho$; (iii) the reflection of (ii) which gives rise to $A^{\prime\left[B_{2}\right]} i F_{3} \delta\left(A^{\left[B_{2}\right]}\right)$; and (iv) the infinite-volume quantity

$$
A^{\prime\left[B_{2}\right]} \frac{i \rho}{2 \omega}\left(\begin{array}{cc}
\frac{2}{3} & -\frac{1}{3}
\end{array}\right)\left(i \mathcal{K}_{\mathrm{df}, 3}\right)\left(\begin{array}{c}
2 / 3 \\
-1 / 3
\end{array}\right) \frac{i \rho}{2 \omega} A^{\left[B_{2}\right]},
$$

which is absorbed by replacing $C_{\infty}^{\left[B_{2}\right]}$ with the alternative infinite-volume quantity defined in Eq. (5.243), below.

To see the general pattern we next consider terms contributing to $C_{L}^{\left[B_{2}\right]}$ that are quadratic in $\mathcal{K}_{\mathrm{df}, 3}$. These arise from either a single $\mathcal{K}_{3, L}^{(u, u)}$ term having two factors of $\mathcal{K}_{\mathrm{df}, 3}$ or two $\mathcal{K}_{3, L}^{(u, u)}$ terms each containing one such factor. Adding these, using the identities (5.226 5.227), and replacing $F$ with $\rho$ where allowed, we find

$$
\begin{aligned}
A^{\prime\left[B_{2}\right]}\left\{i F_{3}\left(\begin{array}{ll}
1 & 1
\end{array}\right)\right. & \left.+\frac{i \rho}{2 \omega}\left(\begin{array}{cc}
\frac{2}{3} & -\frac{1}{3}
\end{array}\right)\right\}\left(i \mathcal{K}_{\mathrm{df}, 3}\right)\left(\begin{array}{c}
1 \\
1
\end{array}\right) \\
\times & \times\left\{F_{3}\left(\begin{array}{ll}
1 & 1
\end{array}\right)+\frac{i \rho}{2 \omega}\left(\begin{array}{cc}
\frac{2}{3} & -\frac{1}{3}
\end{array}\right)\right\}\left(i \mathcal{K}_{\mathrm{df}, 3}\right) \\
& \left.\times\left(\begin{array}{c}
1 \\
1
\end{array}\right) i F_{3}+\left(\begin{array}{c}
2 / 3 \\
-1 / 3
\end{array}\right) \frac{i \rho}{2 \omega}\right\} A^{\left[B_{2}\right]} .
\end{aligned}
$$

Extending this analysis, we find that terms of higher order in $\mathcal{K}_{\mathrm{df}, 3}$ are obtained by inserting additional factors of the matrix

$$
\left(\begin{array}{c}
1 \\
1
\end{array}\right)\left\{i F_{3}\left(\begin{array}{ll}
1 & 1
\end{array}\right)+\frac{i \rho}{2 \omega}\left(\begin{array}{cc}
\frac{2}{3} & -\frac{1}{3}
\end{array}\right)\right\}\left(i \mathcal{K}_{\mathrm{df}, 3}\right)
$$


after the final $\left(\mathcal{K}_{\mathrm{df}, 3}\right)$ in Eq. (5.234).

Our final task is to reorganize the series one last time into infinite-volume kernels separated by finite-volume quantities. This is done by generalizing the analysis described following Eq. (5.228.

The following asymmetric quantities are needed

$$
\begin{gathered}
i \mathcal{K}_{\mathrm{df}, 3}^{x}=\left(\begin{array}{cc}
\frac{2}{3} & -\frac{1}{3}
\end{array}\right)\left(i \mathcal{K}_{\mathrm{df}, 3}\right)\left(\begin{array}{c}
1 \\
1
\end{array}\right), \\
i \mathcal{K}_{\mathrm{df}, 3}^{y}=\left(\begin{array}{cc}
1 & 1
\end{array}\right)\left(i \mathcal{K}_{\mathrm{df}, 3}\right)\left(\begin{array}{c}
2 / 3 \\
-1 / 3
\end{array}\right), \\
i \mathcal{K}_{\mathrm{df}, 3}^{x y}=\left(\begin{array}{cc}
\frac{2}{3} & -\frac{1}{3}
\end{array}\right)\left(i \mathcal{K}_{\mathrm{df}, 3}\right)\left(\begin{array}{c}
2 / 3 \\
-1 / 3
\end{array}\right) .
\end{gathered}
$$

We find a simple geometric series

$$
C_{L}^{\left[B_{2}\right]}=C_{\infty}^{\left[B_{2}, \rho\right]}+\sum_{n=0}^{\infty} A^{\left[B_{2}, \rho\right]}\left[i F_{3} i \mathcal{K}_{\mathrm{df}, 3}^{\left[B_{2}, \rho\right]}\right]^{n} i F_{3} A^{\left[B_{2}, \rho\right]},
$$

where the redefined infinite-volume quantities are

$$
\begin{aligned}
i \mathcal{K}_{\mathrm{df}, 3}^{\left[B_{2}, \rho\right]} & \equiv \sum_{n=0}^{\infty} i \mathcal{K}_{\mathrm{df}, 3}^{\left[B_{2}\right]}\left[\frac{i \rho}{2 \omega} i \mathcal{K}_{\mathrm{df}, 3}^{x}\right]^{n}, \\
A^{\prime\left[B_{2}, \rho\right]} & \equiv \sum_{n=0}^{\infty} A^{\prime\left[B_{2}\right]}\left[\frac{i \rho}{2 \omega} i \mathcal{K}_{\mathrm{df}, 3}^{x}\right]^{n}, \\
A^{\left[B_{2}, \rho\right]} & \equiv\left\{i \mathcal{K}_{\mathrm{df}, 3}^{y} \frac{i \rho}{2 \omega}+i \mathcal{K}_{\mathrm{df}, 3}^{\left[B_{2}, \rho\right]} \frac{i \rho}{2 \omega} i \mathcal{K}_{\mathrm{df}, 3}^{x y}\right\} A^{\left[B_{2}\right]}, \\
C_{\infty}^{\left[B_{2}, \rho\right]} & \equiv C_{\infty}^{\left[B_{2}\right]}+\delta C_{\infty}^{\left[B_{2}\right]}+A^{\left[B_{2}, \rho\right]} \frac{i \rho}{2 \omega} i \mathcal{K}_{\mathrm{df}, 3}^{x y} \frac{i \rho}{2 \omega} A^{\left[B_{2}\right]} .
\end{aligned}
$$

Our notation here is rather compact, with implicit integrals wherever there is a factor of $\rho$, but we stress that it is straightforward to rewrite these definitions as integral equations. We also note that $i \mathcal{K}_{\mathrm{df}, 3}^{\left[B_{2}, \rho\right]}, A^{\prime\left[B_{2}, \rho\right]}$ and $A^{\left[B_{2}, \rho\right]}$ are all symmetric under external particle interchange. This is because they have the vector $(1,1)$, or its transpose, at all ends involving external particles. 
We can bring the result of Eq. (5.239) into familiar form by summing the geometric series, leading to

$$
C_{L}^{\left[B_{2}\right]}=C_{\infty}^{\left[B_{2}, \rho\right]}+A^{\prime\left[B_{2}, \rho\right]} \frac{1}{1-i F_{3} i \mathcal{K}_{\mathrm{df}, 3}^{\left[B_{2}, \rho\right]}} i F_{3} A^{\left[B_{2}, \rho\right]}
$$

This completes the most complicated part of the analysis.

\subsubsection{Including three-to-three insertions}

In this section we add in all diagrams containing three-to-three $\left(B_{3}\right)$ kernels, and so complete the derivation. The new diagrams we are considering are those exemplified by the first and last lines of Fig. 5.4. If there were only $B_{3}$ kernels, with no $B_{2}$ 's, the analysis would be a simple generalization of that for two particles. The complications come from the need to add all possible $B_{2}$ kernels between two $B_{3}$ 's (or between $\sigma$ and a $B_{3}$, or a $B_{3}$ and $\sigma^{\dagger}$ ). A key point here is that the properties of $B_{3}$ are the same as those of $\sigma$ and $\sigma^{\dagger}$, namely that it is symmetric in external momenta (separately on both sides) and is a smooth function of these momenta (within the range of $E$ that we are considering). This means that we can piggyback on the previous analysis in which we added all possible $B_{2}$ 's between $\sigma$ and $\sigma^{\dagger}$.

In particular, a formula analogous to Eq. (5.244) holds for each segment of a diagram between two $B_{3}$ 's (and for that between $\sigma$ and a $B_{3}$, and that between a $B_{3}$ and $\sigma^{\dagger}$ ). In words, Eq. (5.244) tells us that the finite-volume correlator can be written as the sum of an infinite-volume part and a part containing the finite-volume function $F_{3}$. The infinitevolume part is obtained in two stages: first, for each diagram replace all loop sums with $\widetilde{\mathrm{PV}}$ integrals ordered in an appropriate way; second, add in additional terms involving $\rho$, namely those of Eqs. (5.199) and (5.243). In the second term in Eq. (5.244), the endcaps $A^{\prime\left[B_{2}, \rho\right]}$ and $A^{\left[B_{2}, \rho\right]}$ are built up by decorating $\sigma$ and $\sigma^{\dagger}$, respectively, with all possible $B_{2}$ insertions, converting sums to $\widetilde{P V}$ integrals, and then adding in the " $\rho$ terms" of Eqs. 5.241 and 5.242 .

Exactly the same analysis holds for segments of diagrams in which $B_{3}$ 's are playing the role of endcaps. The $B_{3}$ 's are decorated on both sides with $B_{2}$ 's, and can connect to an 
adjacent $B_{3}$ (or $\sigma / \sigma^{\dagger}$ ) either through infinite-volume loops or through a factor of

$$
\mathcal{Z}=\frac{1}{1-i F_{3} i \mathcal{K}_{\mathrm{df}, 3}^{\left[B_{2}, \rho\right]}} i F_{3}
$$

following decoration analogous to that in $A^{\prime\left[B_{2}, \rho\right]}$ and $A^{\left[B_{2}, \rho\right]}$.

To present the result, we first introduce "decoration operators" $D_{C}^{\left[B_{2}, \rho\right]}, D_{A^{\prime}}^{\left[B_{2}, \rho\right]}$ and $D_{A}^{\left[B_{2}, \rho\right]}$, given by

$$
C_{\infty}^{\left[B_{2}\right]} \equiv \sigma D_{C}^{\left[B_{2}, \rho\right]} \sigma^{\dagger}, \quad A^{\prime\left[B_{2}, \rho\right]} \equiv \sigma D_{A^{\prime}}^{\left[B_{2}, \rho\right]}, \quad \text { and } \quad A^{\left[B_{2}, \rho\right]} \equiv D_{A}^{\left[B_{2}, \rho\right]} \sigma^{\dagger}
$$

These are infinite-volume integral operators defined implicitly by the work of previous subsections. This allows us to write Eq. 5.244 as

$$
C_{L}^{\left[B_{2}\right]}=\sigma\left\{D_{C}^{\left[B_{2}, \rho\right]}+D_{A^{\prime}}^{\left[B_{2}, \rho\right]} \mathcal{Z} D_{A}^{\left[B_{2}, \rho\right]}\right\} \sigma^{\dagger}
$$

The reason for using this notation is that it works also for segments of diagrams involving $B_{3}$ 's at the ends. Thus, for example, a segment of the finite-volume correlator between two $B_{3}$ 's can be written

$$
\ldots B_{3}\left\{D_{C}^{\left[B_{2}, \rho\right]}+D_{A^{\prime}}^{\left[B_{2}, \rho\right]} \mathcal{Z} D_{A}^{\left[B_{2}, \rho\right]}\right\} B_{3} \cdots
$$

The key point is that the same decoration operators appear as in (5.247).

We can now write down the result for the full finite-volume correlator

$$
\begin{aligned}
C_{L}=\sigma\left\{D_{C}^{\left[B_{2}, \rho\right]}+D_{A^{\prime}}^{\left[B_{2}, \rho\right]} \mathcal{Z} D_{A}^{\left[B_{2}, \rho\right]}\right\} \sigma^{\dagger} & \\
+\sigma\left\{D_{C}^{\left[B_{2}, \rho\right]}+D_{A^{\prime}}^{\left[B_{2}, \rho\right]} \mathcal{Z} D_{A}^{\left[B_{2}, \rho\right]}\right\} & i B_{3}\left\{D_{C}^{\left[B_{2}, \rho\right]}+D_{A^{\prime}}^{\left[B_{2}, \rho\right]} \mathcal{Z} D_{A}^{\left[B_{2}, \rho\right]}\right\} \sigma^{\dagger} \\
+\sigma\left\{D_{C}^{\left[B_{2}, \rho\right]}+D_{A^{\prime}}^{\left[B_{2}, \rho\right]} \mathcal{Z} D_{A}^{\left[B_{2}, \rho\right]}\right\} i B_{3}\{ & \left.D_{C}^{\left[B_{2}, \rho\right]}+D_{A^{\prime}}^{\left[B_{2}, \rho\right]} \mathcal{Z} D_{A}^{\left[B_{2}, \rho\right]}\right\} i B_{3} \\
& \times\left\{D_{C}^{\left[B_{2}, \rho\right]}+D_{A^{\prime}}^{\left[B_{2}, \rho\right]} \mathcal{Z} D_{A}^{\left[B_{2}, \rho\right]}\right\} \sigma^{\dagger}+\ldots
\end{aligned}
$$

As in the previous subsection, this can be reorganized into the form

$$
C_{L}=C_{\infty}+\sum_{n=0}^{\infty} A^{\prime}\left[\mathcal{Z} i B_{3}^{\left[B_{2}, \rho\right]}\right]^{n} \mathcal{Z} A
$$


where

$$
\begin{aligned}
i B_{3}^{\left[B_{2}, \rho\right]} & =\sum_{n=0}^{\infty} D_{A}^{\left[B_{2}, \rho\right]}\left[i B_{3} D_{C}^{\left[B_{2}, \rho\right]}\right]^{n} i B_{3} D_{A^{\prime}}^{\left[B_{2}, \rho\right]}, \\
A^{\prime} & =\sum_{n=0}^{\infty} \sigma\left[D_{C}^{\left[B_{2}, \rho\right]} i B_{3}\right]^{n} D_{A^{\prime}}^{\left[B_{2}, \rho\right]} \\
A & =\sum_{n=0}^{\infty} D_{A}^{\left[B_{2}, \rho\right]}\left[i B_{3} D_{C}^{\left[B_{2}, \rho\right]}\right]^{n} \sigma^{\dagger} \\
C_{\infty} & =\sum_{n=0}^{\infty} \sigma D_{C}^{\left[B_{2}, \rho\right]}\left[i B_{3} D_{C}^{\left[B_{2}, \rho\right]}\right]^{n} \sigma^{\dagger}
\end{aligned}
$$

The latter three equations give the final forms of the endcaps and the infinite-volume correlator, now including all factors of $B_{3}$.

We can now sum the geometric series in Eq. (5.250) and perform some simple algebraic manipulations to bring the result to its final form

$$
C_{L}=C_{\infty}+A^{\prime} \frac{1}{1-i F_{3} i \mathcal{K}_{\mathrm{df}, 3}} i F_{3} A
$$

where

$$
\mathcal{K}_{\mathrm{df}, 3} \equiv \mathcal{K}_{\mathrm{df}, 3}^{\left[B_{2}, \rho\right]}+B_{3}^{\left[B_{2}, \rho\right]}
$$

is the full divergence-free three-to-three amplitude.

We close our derivation by returning to an issue raised in the introduction to this section, namely the possibility of poles in $A, A^{\prime}$ and $C_{\infty}$. We argue that, while such poles can be present, they cannot contribute to the finite-volume spectrum, i.e. they do not lead to poles in $C_{L}$. Only solutions to the quantization condition (5.18) lead to poles in $C_{L}$.

The intuitive argument for this result is that $A, A^{\prime}$ and $C_{\infty}$ are infinite volume quantities. While they are non-standard, being defined with the $\widetilde{\mathrm{PV}}$ prescription and involving the decoration described above, they have no dependence on $L$. Thus, if they did lead to poles in $C_{L}$, this would imply states in the finite-volume spectrum whose energies were independent of $L$ (up to corrections of the form $\exp (-m L)$ ). The only plausible state with this property is a single particle, but this is excluded by our choice of energy range $\left(m<E^{*}<5 m\right)$. Threeparticle bound states will have finite-volume corrections that are exponentially suppressed by $\exp (-\gamma L)$, with $\gamma \ll m$ the binding momentum, but these should be captured by our 
analysis, just as is the case for two-particle bound states [151]. Finally, above-threshold "scattering" states should have energies with power-law dependence on $L$. This is true in the two-particle case, and we expect it to continue to hold for three particles. This is confirmed, for example, by the analysis of three (and more) particles using non-relativistic quantum mechanics [245, 246].

For the two-particle analysis this argument can be made more rigorous, and it is informative to see how this works. We have recalled the two-particle quantization condition in Sec. 5.3.1, and give here the form of the corresponding two-particle finite-volume correlator:

$$
C_{L, 2}=C_{\infty, 2}+i A_{2}^{\prime} \frac{1}{1+F \mathcal{K}_{2}} F A_{2}
$$

The subscripts on $A, A^{\prime}$ and $C$ indicate that these are the two-particle endcaps and correlator, while $F$ is defined in Eqs. (5.24)-(5.24) (although here we drop the spectator momentum index).

What we now show is that there are poles in $A_{2}, A_{2}^{\prime}$ and $C_{\infty, 2}$, but these cancel in $C_{L, 2}$. To see this we use the freedom to arbitrarily choose the interpolating functions $\sigma$ and $\sigma^{\dagger}$ without affecting the position of poles in $C_{L, 2}$. Specifically, we set both $\sigma$ and $\sigma^{\dagger}$ equal to the two-particle Bethe-Salpeter kernel $i B_{2}$, which, we recall, is a smooth non-singular function. One then finds that

$$
C_{\infty, 2}=i \mathcal{K}_{2}-i B_{2} \text { and } A_{2}=A_{2}^{\prime}=\mathcal{K}_{2}
$$

Inserting these results into Eq. (257) we find that

$$
C_{L, 2}=-i B_{2}+i \mathcal{K}_{2}+i \mathcal{K}_{2} \frac{1}{1-i F i \mathcal{K}_{2}} i F i \mathcal{K}_{2}=-i B_{2}+\frac{i}{\mathcal{K}_{2}^{-1}+F}
$$

From this we draw two conclusions. First, $A_{2}, A_{2}^{\prime}$ and $C_{\infty, 2}$ have poles whenever $\mathcal{K}_{2}$ diverges. Such poles occur, for a given angular momentum, when $\delta_{\ell}=\pi / 2 \bmod \pi$. Thus, using the $\widetilde{\mathrm{PV}}$ prescription, there are, in general, poles in $A_{2}, A_{2}^{\prime}$ and $C_{\infty, 2}$. Second, these poles cancel in $C_{L, 2}$, as shown by the second form in Eq. (5.259), which is clearly finite when $\mathcal{K}_{2}$ diverges.

We suspect that a similar result holds for the three-particle analysis, but have not yet been able to demonstrate this. Thus, in the three-particle case we must rely for now on the intuitive argument given above. 


\subsection{Conclusion of three-particle analysis}

In this chapter we have presented and derived a quantization condition that relates the finitevolume spectrum to two-to-two and three-to-three infinite-volume scattering quantities. In Section 5.1 we have explained this relation in detail, so that one may use this section alone to understand our result. Also in this section, we have explained that on-shell divergences in the three-to-three scattering amplitude make it an unsuitable quantity to directly extract from the finite-volume spectrum. This observation, together with other technical complications explained in the derivation, led us to a result which depends on a non-standard, infinitevolume three-to-three K-matrix, denoted $\mathcal{K}_{\mathrm{df}, 3}$.

This three-particle K-matrix is a smooth-function of three-particle phase space, and thus has a uniformly convergent partial wave expansion. In Section 5.2 we have explained how approximating $\mathcal{K}_{\mathrm{df}, 3}$ (as well as the two-particle K-matrix) with a truncated partial-wave expansion leads to a quantization condition with a finite number of free parameters. This shows that our quantization condition, like Lüscher's well known two-particle condition, may be practically useful in extracting scattering information.

The bulk of this work is the derivation, presented in Section 5.3. In this section we have introduced a skeleton expansion which describes an arbitrary relativistic field theory with a single scalar particle that interacts according to any even-legged vertices. We have then analyzed all diagrams in the expansion in finite-volume, dividing the derivation into subsections according to the types of diagrams considered. We have shown how summing all diagrams gives our main result.

In future work we plan to demonstrate the utility of our result in various applications. First, in a forthcoming note, we present a perturbative expansion of our quantization condition in powers of $1 / L$. This expansion agrees with existing expansions that were calculated in Refs. [245, 246] using non-relativistic quantum mechanics, and provides a nontrivial check on our relation. Next we aim to relate our three-particle infinite-volume quantity $\mathcal{K}_{\mathrm{df}, 3}$ to more standard scattering observables. This will require converting the non-standard poleprescription used in all Feynman diagrams, to the standard $i \epsilon$ prescription. The analysis is expected to be similar to the derivation presented here, but a careful study is required. 


\section{Chapter 6}

\section{CONCLUSION}

In this thesis we have shown how one may relate the finite-volume spectrum and finitevolume matrix elements to various multi-hadron infinite-volume observables. These relations are of importance to LQCD because only finite-volume quantities are accessible in numerical LQCD calculations. Indeed, the formalism presented here provides the only known method to systemically determine multi-hadron observables from the underlying theory.

The ultimate test of these ideas is to apply them to finite-volume data. For the formalism presented in Chapter 2, this has already been achieved with a benchmark calculation of the $\pi K-\eta K$ system by Ref. [92]. For the formalism of Chapters 3-5 this remains to be completed. This will be a particular challenge for the formalism of Chapter 5. Because three-particle phase space is more complicated than that for two-particles, extracting scattering information from the spectrum in this sector is more challenging. However, the work presented above shows that this is possible in principle, and given the success in the complicated $\pi K-\eta K$ system, we are encouraged that, also for three particles, LQCD can contribute physical information. 


\section{BIBLIOGRAPHY}

[1] David Griffiths, Intoduction to Elementary Particles. 1987.

[2] J.J. Thomson, Cathode Rays, The Electrician 39 (1897) 104.

[3] J.J. Thomson, Cathode Rays, Philosophical Magazine 44 (1897) 293.

[4] J. Chadwick, The Existence of a Neutron, Proc. R. Soc. Lond. A 136830 (1932) 692-708.

[5] M. Planck, On the Law of Distribution of Energy in the Normal Spectrum, Annalen der Physik 4 (3) (1901) 553-563.

[6] A. Einstein, ber einen die Erzeugung und Verwandlung des Lichtes betreffenden heuristischen Gesichtspunkt, Annalen der Physik 17 (6) (1905) 132-148.

[7] A. Einstein, ber die Entwicklung unserer Anschauungen ber das Wesen und die Konstitution der Strahlung, Physikalische Zeitschrift 10 (1909) 817-825.

[8] A. Einstein, Zur Quantentheorie der Strahlung, Physikalische Zeitschrift 18 (1917) $121-128$.

[9] A. Compton, A Quantum Theory of the Scattering of X-rays by Light Elements, Physical Review 21 (5) (1923) 483502.

[10] P.A.M. Dirac, The Quantum Theory of the Electron, Proc. R. Soc. Lond. A 117 (1928).

[11] P.A.M. Dirac, A Theory of Electrons and Protons, Proc. R. Soc. Lond. A 126 (1930).

[12] C. D. Anderson, The Positive Electron, Physical Review 43 (6) (1932) 491-494.

[13] E. Fermi, Versuch einer Theorie der-Strahlen. I, Zeitschrift fr Physik A $8 \mathbf{8}$ (34) (1934) 161.

[14] E. Fermi (translated by F. L. Wilson), Fermi's Theory of Beta Decay, American Journal of Physics 35 (12) (1968) 1150.

[15] H. Yukawa Proc. Physico-Math. Soc. Japan 17 (1935) 48. 
[16] S. Neddermeyer and C. Anderson Physical Review 51 (1937) 884.

[17] S. Neddermeyer and C. Anderson Physical Review 54 (1938) 88.

[18] J. Street and E. Stevenson Physical Review (L) 52 (1937) 1003.

[19] C. Lattes, H. Muirhead, G. Occhialini, and C. Powell Nature 159 (1947) 694.

[20] C. Lattes, H. Muirhead, G. Occhialini, and C. Powell Nature 160 (1947) 454.

[21] G. Rochester and C. Butler, Evidence for the Existence of New Unstable Elementary Particles, Nature 160 (1947) 855-857.

[22] Willis Lamb, Les Prix Nobel en 1955. Fine Structure of the Hydrogen Atom. Norstedt and Soner, 1 ed., 1956.

[23] Silvan S. Schweber, QED and the Men Who Made It. Princeton University Press, 1 ed., 1994.

[24] S. Tomonaga, On a relativistically invariant formulation of the quantum theory of wave fields, Prog.Theor.Phys. 1 (1946) 27-42.

[25] J. S. Schwinger, On Quantum electrodynamics and the magnetic moment of the electron, Phys.Rev. 73 (1948) 416-417.

[26] J. S. Schwinger, Quantum electrodynamics. I A covariant formulation, Phys.Rev. 74 (1948) 1439.

[27] R. Feynman, Space - time approach to quantum electrodynamics, Phys.Rev. 76 (1949) 769-789.

[28] R. Feynman, The Theory of positrons, Phys.Rev. 76 (1949) 749-759.

[29] R. Feynman, Mathematical formulation of the quantum theory of electromagnetic interaction, Phys.Rev. 80 (1950) 440-457.

[30] F. Dyson, The Radiation theories of Tomonaga, Schwinger, and Feynman, Phys.Rev. 75 (1949) 486-502.

[31] F. Dyson, The S matrix in quantum electrodynamics, Phys.Rev. 75 (1949) $1736-1755$.

[32] S. Glashow, Partial Symmetries of Weak Interactions, Nucl.Phys. 22 (1961) 579-588. 
[33] S. Weinberg, A Model of Leptons, Phys.Rev.Lett. 19 (1967) 1264-1266.

[34] A. Salam, Elementary Particle Physics: Relativistic Groups and Analyticity, Eighth Nobel Symposium, N. Svartholm (eds.) Stockholm (1968) 367.

[35] F. Englert and R. Brout, Broken Symmetry and the Mass of Gauge Vector Mesons, Phys.Rev.Lett. 13 (1964) 321-323.

[36] P. W. Higgs, Broken Symmetries and the Masses of Gauge Bosons, Phys.Rev.Lett. 13 (1964) 508-509.

[37] G. Guralnik, C. Hagen, and T. Kibble, Global Conservation Laws and Massless Particles, Phys.Rev.Lett. 13 (1964) 585-587.

[38] UA1 Collaboration Collaboration, G. Arnison et al., Experimental Observation of Isolated Large Transverse Energy Electrons with Associated Missing Energy at $s^{* *}(1 / 2)=540-G e V$, Phys.Lett. B122 (1983) 103-116.

[39] UA1 Collaboration Collaboration, G. Arnison et al., Experimental Observation of Lepton Pairs of Invariant Mass Around 95-GeV/c**2 at the CERN SPS Collider, Phys.Lett. B126 (1983) 398-410.

[40] ATLAS Collaboration Collaboration, G. Aad et al., Observation of a new particle in the search for the Standard Model Higgs boson with the ATLAS detector at the LHC, Phys.Lett. B716 (2012) 1-29, arXiv:1207.7214.

[41] CMS Collaboration Collaboration, S. Chatrchyan et al., Observation of a new boson at a mass of $125 \mathrm{GeV}$ with the CMS experiment at the LHC, Phys.Lett. B716 (2012) 30-61, arXiv:1207.7235.

[42] W. Heisenberg, Über den Bau der Atomkerne. I, Zeitschrift fur Physik $7 \mathbf{7}$ (Jan., 1932) $1-11$.

[43] E. Wigner, On the Consequences of the Symmetry of the Nuclear Hamiltonian on the Spectroscopy of Nuclei, Phys.Rev. 51 (1937) 106-119.

[44] T. Nakano and K. Nishijima, Charge Independence for V-particles, Prog.Theor.Phys. 10 (1953) 581-582.

[45] K. Nishijima, Charge Independence Theory of V Particles, Prog.Theor.Phys. 13.

[46] M. Gell-Mann, The interpretation of the new particles as displaced charge multiplets, Nuovo Cim. 4 (1956), no. S2 848-866. 
[47] M. Gell-Mann, A Schematic Model of Baryons and Mesons, Phys.Lett. 8 (1964) $214-215$.

[48] G. Zweig, An SU(3) model for strong interaction symmetry and its breaking. Version 1, .

[49] G. Zweig, An SU(3) model for strong interaction symmetry and its breaking. Version 2, .

[50] James Joyce, Finnegan's Wake. Faber and Faber, 1939.

[51] F. Tkachov, A Contribution to the history of quarks: Boris Struminsky's 1965 JINR publication, arXiv:0904.0343.

[52] M. Han and Y. Nambu, Three Triplet Model with Double SU(3) Symmetry, Phys.Rev. 139 (1965) B1006-B1010.

[53] O. Greenberg, Spin and Unitary Spin Independence in a Paraquark Model of Baryons and Mesons, Phys.Rev.Lett. 13 (1964) 598-602.

[54] C.-N. Yang and R. L. Mills, Conservation of Isotopic Spin and Isotopic Gauge Invariance, Phys.Rev. 96 (1954) 191-195.

[55] J. Bjorken, Asymptotic Sum Rules at Infinite Momentum, Phys.Rev. 179 (1969) $1547-1553$.

[56] E. D. Bloom, D. Coward, H. DeStaebler, J. Drees, G. Miller, et al., High-Energy Inelastic e p Scattering at 6-Degrees and 10-Degrees, Phys.Rev.Lett. 23 (1969) 930-934.

[57] M. Breidenbach, J. I. Friedman, H. W. Kendall, E. D. Bloom, D. Coward, et al., Observed Behavior of Highly Inelastic electron-Proton Scattering, Phys.Rev.Lett. 23 (1969) 935-939.

[58] SLAC-SP-017 Collaboration Collaboration, J. Augustin et al., Discovery of a Narrow Resonance in e+ e- Annihilation, Phys.Rev.Lett. 33 (1974) 1406-1408.

[59] J. Aubert, U. Becker, P. Biggs, J. Burger, M. Chen, et al., Discovery of the New Particle J, Nucl.Phys. B89 (1975) 1.

[60] S. Herb, D. Hom, L. Lederman, J. Sens, H. Snyder, et al., Observation of a Dimuon Resonance at 9.5-GeV in 400-GeV Proton-Nucleus Collisions, Phys.Rev.Lett. 39 (1977) 252-255. 
[61] CDF Collaboration Collaboration, F. Abe et al., Observation of top quark production in $\bar{p} p$ collisions, Phys.Rev.Lett. 74 (1995) 2626-2631, hep-ex/9503002.

[62] Do Collaboration Collaboration, S. Abachi et al., Search for high mass top quark production in $p \bar{p}$ collisions at $\sqrt{s}=1.8$ TeV, Phys.Rev.Lett. 74 (1995) 2422-2426, hep-ex/9411001.

[63] D. J. Gross and F. Wilczek, Ultraviolet Behavior of Nonabelian Gauge Theories, Phys.Rev.Lett. 30 (1973) 1343-1346.

[64] H. D. Politzer, Reliable Perturbative Results for Strong Interactions?, Phys.Rev.Lett. 30 (1973) 1346-1349.

[65] G. 't Hooft, Renormalization of Massless Yang-Mills Fields, Nucl.Phys. B33 (1971) 173-199.

[66] DESY-Hamburg-Heidelberg-Munich Collaboration Collaboration, F. Heimlich et al., Hadronic Jets From $e^{+} e^{-}$Annihilations in the $\Upsilon$ and $\Upsilon^{\prime}$ Region, Phys.Lett. B86 (1979) 399.

[67] D. Barber, U. Becker, H. Benda, A. Boehm, J. Branson, et al., Discovery of Three Jet Events and a Test of Quantum Chromodynamics at PETRA Energies, Phys.Rev.Lett. 43 (1979) 830.

[68] M. Srednicki, Quantum Field Theory. Cambridge University Press, 2007.

[69] Particle Data Group Collaboration, J. Beringer et al., Review of Particle Physics (RPP), Phys.Rev. D86 (2012) 010001.

[70] L. Lellouch and M. Luscher, Weak transition matrix elements from finite volume correlation functions, Commun.Math.Phys. 219 (2001) 31-44, hep-lat/0003023.

[71] M. Gell-Mann and A. Pais Proceeding Glasgow Conf. 1954, Pergamon London (1954) 342.

[72] R.W. Birge et al. Nuovo Cimento 4 (1956) 834.

[73] G. Alexander et al. Nuovo Cimento 6 (1957) 478.

[74] T. Hambye, G. Kohler, and P. Soldan, New analysis of the Delta $I=1 / 2$ rule in kaon decays and the B-affine(K) parameter, Eur.Phys.J. C10 (1999) 271-292, hep-ph/9902334. 
[75] M. Gaillard and B. W. Lee, Delta $I=1 / 2$ Rule for Nonleptonic Decays in Asymptotically Free Field Theories, Phys.Rev.Lett. 33 (1974) 108.

[76] G. Altarelli and L. Maiani, Octet Enhancement of Nonleptonic Weak Interactions in Asymptotically Free Gauge Theories, Phys.Lett. B52 (1974) 351-354.

[77] M. A. Shifman, A. Vainshtein, and V. I. Zakharov, Light Quarks and the Origin of the Delta $I=1 / 2$ Rule in the Nonleptonic Decays of Strange Particles, Nucl.Phys. B120 (1977) 316.

[78] F. J. Gilman and M. B. Wise, Effective Hamiltonian for Delta $s=1$ Weak Nonleptonic Decays in the Six Quark Model, Phys.Rev. D20 (1979) 2392.

[79] B. Guberina and R. Peccei, Quantum Chromodynamic Effects and CP Violation in the Kobayashi-Maskawa Model, Nucl.Phys. B163 (1980) 289.

[80] A. J. Buras, M. Jamin, M. E. Lautenbacher, and P. H. Weisz, Two loop anomalous dimension matrix for Delta $S=1$ weak nonleptonic decays. 1. O(alpha-s**2), Nucl.Phys. B400 (1993) 37-74, hep-ph/9211304.

[81] A. J. Buras, M. Jamin, and M. E. Lautenbacher, Two loop anomalous dimension matrix for Delta $S=1$ weak nonleptonic decays. 2. O(alpha-alpha-s), Nucl.Phys. B400 (1993) 75-102, hep-ph/9211321.

[82] A. J. Buras, M. Jamin, and M. E. Lautenbacher, The Anatomy of epsilon-prime / epsilon beyond leading logarithms with improved hadronic matrix elements, Nucl.Phys. B408 (1993) 209-285, hep-ph/9303284].

[83] M. Ciuchini, E. Franco, G. Martinelli, and L. Reina, epsilon-prime / epsilon at the Next-to-leading order in QCD and QED, Phys.Lett. B301 (1993) 263-271, hep-ph/9212203.

[84] B. W. Lee, J. Primack, and S. Treiman, Some physical constraints on gauge models of weak interactions, Phys.Rev. D7 (1973) 510-516.

[85] M. Gaillard and B. W. Lee, Rare Decay Modes of the K-Mesons in Gauge Theories, Phys.Rev. D10 (1974) 897.

[86] G. 't Hooft, A Two-Dimensional Model for Mesons, Nucl.Phys. B75 (1974) 461.

[87] W. A. Bardeen, A. Buras, and J. Gerard, A Consistent Analysis of the Delta I= $1 / 2$ Rule for K Decays, Phys.Lett. B192 (1987) 138. 
[88] RBC, UKQCD Collaboration, P. Boyle et al., Emerging understanding of the $\Delta I=1 / 2$ Rule from Lattice QCD, Phys.Rev.Lett. 110 (2013), no. 15 152001, arXiv:1212.1474.

[89] N. H. Christ, Calculating the two-pion decay and mixing of neutral $K$ mesons, PoS LATTICE2012 (2012) 008, arXiv:1301.4239.

[90] T. Janowski, C. Sachrajda, P. Boyle, N. Christ, R. Mawhinney, et al., Determination of the $A_{2}$ amplitude of $K \rightarrow \pi \pi$ decays, arXiv:1311.3844.

[91] T. Blum, P. Boyle, N. Christ, N. Garron, E. Goode, et al., The $K \rightarrow(\pi \pi)_{I=2}$ Decay Amplitude from Lattice QCD, arXiv:1111.1699.

[92] J. J. Dudek, R. G. Edwards, C. E. Thomas, and D. J. Wilson, Resonances in coupled $\pi K, \eta K$ scattering from quantum chromodynamics, arXiv:1406.4158.

[93] R. L. Jaffe, Multi-Quark Hadrons. 1. The Phenomenology of (2 Quark 2 anti-Quark) Mesons, Phys.Rev. D15 (1977) 267.

[94] D. Black, A. H. Fariborz, F. Sannino, and J. Schechter, Putative light scalar nonet, Phys.Rev. D59 (1999) 074026, hep-ph/9808415.

[95] N. Achasov and A. Kiselev, The New analysis of the KLOE data on the $\phi \rightarrow \eta \pi^{0} \gamma$ decay, Phys.Rev. D68 (2003) 014006, hep-ph/0212153.

[96] J. Pelaez, Light scalars as tetraquarks or two-meson states from large $N(c)$ and unitarized chiral perturbation theory, Mod.Phys.Lett. A19 (2004) 2879-2894, hep-ph/0411107.

[97] J. D. Weinstein and N. Isgur, Do Multi-Quark Hadrons Exist?, Phys.Rev.Lett. 48 (1982) 659.

[98] J. Oller, E. Oset, and J. Pelaez, Nonperturbative approach to effective chiral Lagrangians and meson interactions, Phys.Rev.Lett. 80 (1998) 3452-3455, hep-ph/9803242.

[99] LHCb Collaboration Collaboration, R. Aaij et al., Evidence for CP violation in time-integrated $D^{0} \rightarrow h^{-} h^{+}$decay rates, Phys.Rev.Lett. 108 (2012) 111602, arXiv:1112.0938.

[100] M. Golden and B. Grinstein, Enhanced CP Violations in Hadronic Charm Decays, Phys.Lett. B222 (1989) 501. 
[101] G. Isidori, J. F. Kamenik, Z. Ligeti, and G. Perez, Implications of the LHCb Evidence for Charm CP Violation, arXiv:1111.4987.

[102] J. Brod, A. L. Kagan, and J. Zupan, On the size of direct CP violation in singly Cabibbo-suppressed D decays, arXiv:1111.5000.

[103] B. Bhattacharya, M. Gronau, and J. L. Rosner, CP asymmetries in singly-Cabibbo-suppressed D decays to two pseudoscalar mesons, arXiv:1201.2351.

[104] E. Franco, S. Mishima, and L. Silvestrini, The Standard Model confronts CP violation in $D^{0} \rightarrow \pi^{+} \pi^{-}$and $D^{0} \rightarrow K^{+} K^{-}$, arXiv:1203.3131.

[105] J. Brod, Y. Grossman, A. L. Kagan, and J. Zupan, A Consistent Picture for Large Penguins in $D^{0} \rightarrow \pi^{+} \pi^{-}, K^{+} K^{-}$, JHEP 1210 (2012) 161, [arXiv:1203.6659].

[106] LHCb collaboration Collaboration, R. Aaij et al., Measurement of CP asymmetry in $D^{0} \rightarrow K^{-} K^{+}$and $D^{0} \rightarrow \pi^{-} \pi^{+}$decays, JHEP 1407 (2014) 041, arXiv:1405.2797.

[107] MuSun Collaboration Collaboration, V. Andreev et al., Muon Capture on the Deuteron - The MuSun Experiment, arXiv:1004.1754.

[108] S. Ando, T. Park, K. Kubodera, and F. Myhrer, The mu-d capture rate in effective field theory, Phys.Lett. B533 (2002) 25-36, nucl-th/0109053.

[109] G. Bardin, J. Duclos, J. Martino, A. Bertin, M. Capponi, M. Piccinini, and A. Vitale, A measurement of the muon capture rate in liquid deuterium by the lifetime technique, Nucl. Phys. A453 (1986) 591-604.

[110] M. t. Cargnelli. In Proceedings of the XXIII Yamada Conf. on Nuclear Weak Processes and Nuclear Structure, Osaka, Japan, 1989.

[111] J.-W. Chen, T. Inoue, X.-d. Ji, and Y.-c. Li, Fixing two-nucleon weak-axial coupling L(1,A) from mu-d capture, Phys.Rev. C72 (2005) 061001, nucl-th/0506001.

[112] BELLE Collaboration Collaboration, J.-T. Wei et al., Measurement of the Differential Branching Fraction and Forward-Backword Asymmetry for $B \rightarrow K(*) l+l-$, Phys.Rev.Lett. 103 (2009) 171801, arXiv:0904.0770.

[113] CDF Collaboration Collaboration, T. Aaltonen et al., Measurements of the Angular Distributions in the Decays $B \rightarrow K^{(*)} \mu^{+} \mu^{-}$at CDF, Phys.Rev.Lett. 108 (2012) 081807, arXiv:1108.0695. 
[114] BaBar Collaboration Collaboration, J. Lees et al., Measurement of Branching Fractions and Rate Asymmetries in the Rare Decays $B \rightarrow K^{(*)} l^{+} l^{-}$, Phys.Rev. D86 (2012) 032012, arXiv:1204.3933.

[115] LHCb Collaboration Collaboration, R. Aaij et al., Differential branching fraction and angular analysis of the decay $B^{0} \rightarrow K^{* 0} \mu^{+} \mu^{-}$, JHEP 1308 (2013) 131, arXiv:1304.6325.

[116] LHCb collaboration Collaboration, R. Aaij et al., Measurement of Form-Factor-Independent Observables in the Decay $B^{0} \rightarrow K^{* 0} \mu^{+} \mu^{-}$, Phys.Rev.Lett. 111 (2013), no. 19 191801, arXiv:1308.1707].

[117] C. Bobeth, G. Hiller, and D. van Dyk, General Analysis of $\bar{B} \rightarrow \bar{K}^{(*)} \ell^{+} \ell^{-}$Decays at Low Recoil, Phys.Rev. D87 (2013) 034016, arXiv:1212.2321.

[118] S. Descotes-Genon, J. Matias, and J. Virto, Understanding the $B \rightarrow K^{*} \mu^{+} \mu^{-}$ Anomaly, Phys.Rev. D88 (2013) 074002, arXiv:1307.5683.

[119] C. Hambrock, G. Hiller, S. Schacht, and R. Zwicky, $B \rightarrow K^{*}$ Form Factors from Flavor Data to QCD and Back, arXiv:1308.4379.

[120] F. Beaujean, C. Bobeth, and D. van Dyk, Comprehensive Bayesian Analysis of Rare (Semi)leptonic and Radiative B Decays, arXiv:1310.2478

[121] N. Isgur and G. Karl, Hyperfine Interactions in Negative Parity Baryons, Phys.Lett. B72 (1977) 109.

[122] N. Isgur and G. Karl, Positive Parity Excited Baryons in a Quark Model with Hyperfine Interactions, Phys.Rev. D19 (1979) 2653.

[123] H. B. Meyer, Photodisintegration of a Bound State on the Torus, arXiv:1202.6675.

[124] M. Bertero, P. Boccacci, and E. R. Pike, On the Recovery and Resolution of Exponential Relaxation Rates from Experimental Data: A Singular-Value Analysis of the Laplace Transform Inversion in the Presence of Noise, Proc. R. Soc. Lond. A383 (1982).

[125] X.-d. Ji and C.-w. Jung, Studying hadronic structure of the photon in lattice QCD, Phys.Rev.Lett. 86 (2001) 208, |hep-lat/0101014].

[126] L. Maiani and M. Testa, Final state interactions from Euclidean correlation functions, Phys.Lett. B245 (1990) 585-590. 
[127] M. Luscher, Volume Dependence of the Energy Spectrum in Massive Quantum Field Theories. 2. Scattering States, Commun.Math.Phys. 105 (1986) 153-188.

[128] M. Luscher, Two particle states on a torus and their relation to the scattering matrix, Nucl.Phys. B354 (1991) 531-578.

[129] K. Rummukainen and S. A. Gottlieb, Resonance scattering phase shifts on a nonrest frame lattice, Nucl. Phys. B450 (1995) 397-436, hep-lat/9503028.

[130] C. Kim, C. Sachrajda, and S. R. Sharpe, Finite-volume effects for two-hadron states in moving frames, Nucl.Phys. B727 (2005) 218-243, hep-lat/0507006.

[131] N. H. Christ, C. Kim, and T. Yamazaki, Finite volume corrections to the two-particle decay of states with non-zero momentum, Phys.Rev. D72 (2005) 114506 , hep-lat/0507009].

[132] C. Liu, X. Feng, and S. He, Two particle states in a box and the S-matrix in multi-channel scattering, Int.J.Mod.Phys. A21 (2006) 847-850, hep-lat/0508022.

[133] M. Lage, U.-G. Meissner, and A. Rusetsky, A Method to measure the antikaon-nucleon scattering length in lattice QCD, Phys.Lett. B681 (2009) 439-443, arXiv:0905.0069].

[134] V. Bernard, M. Lage, U.-G. Meissner, and A. Rusetsky, Scalar mesons in a finite volume, JHEP 1101 (2011) 019, arXiv:1010.6018.

[135] M. Doring, U.-G. Meissner, E. Oset, and A. Rusetsky, Unitarized Chiral Perturbation Theory in a finite volume: Scalar meson sector, Eur.Phys.J. A47 (2011) 139, arXiv:1107.3988.

[136] A. Agadjanov, V. Bernard, U.-G. Meiner, and A. Rusetsky, A framework for the calculation of the $\Delta N \gamma^{*}$ transition form factor on the lattice, arXiv:1405.3476.

[137] L. Roca and E. Oset, Scattering of unstable particles in a finite volume: the case of $\pi \rho$ scattering and the $a_{1}(1260)$ resonance, Phys.Rev. D85 (2012) 054507, arXiv: 1201.0438.

[138] K. Polejaeva and A. Rusetsky, Three particles in a finite volume, Eur.Phys.J. A48 (2012) 67, arXiv:1203.1241.

[139] R. A. Briceno and Z. Davoudi, Three-particle scattering amplitudes from a finite volume formalism, Phys.Rev. D87 (2012) 094507, arXiv:1212.3398. 
[140] M. T. Hansen and S. R. Sharpe, Multiple-channel generalization of Lellouch-Luscher formula, Phys.Rev. D86 (2012) 016007, arXiv:1204.0826.

[141] M. Luscher, Volume Dependence of the Energy Spectrum in Massive Quantum Field Theories. 1. Stable Particle States, Commun.Math.Phys. 104 (1986) 177.

[142] M. Luescher, Signatures of unstable particles in finite volume, Nucl. Phys. B364 (1991) 237-254.

[143] Z. Davoudi and M. J. Savage, Improving the Volume Dependence of Two-Body Binding Energies Calculated with Lattice QCD, Phys.Rev. D84 (2011) 114502, arXiv:1108.5371.

[144] Z. Fu, Rummukainen-Gottlieb's formula on two-particle system with different mass, Phys.Rev. D85 (2012) 014506, arXiv:1110.0319.

[145] L. Leskovec and S. Prelovsek, Scattering phase shifts for two particles of different mass and non-zero total momentum in lattice QCD, Phys.Rev. D85 (2012) 114507, arXiv:1202.2145.

[146] R. A. Briceno and Z. Davoudi, Moving multichannel systems in a finite volume with application to proton-proton fusion, Phys. Rev. D. 88, 094507 (2013) 094507, arXiv:1204.1110.

[147] J. M. Blatt and L. C. Biedenharn Phys. Rev. 86 (1952) 399.

[148] C. D. Lin, G. Martinelli, C. T. Sachrajda, and M. Testa, $K-\dot{z}$ pi pi decays in a finite volume, Nucl.Phys. B619 (2001) 467-498, hep-lat/0104006.

[149] R. A. Briceo, M. T. Hansen, and A. Walker-Loud, Multichannel one-to-two transition form factors in a finite volume, arXiv:1406.5965.

[150] S. Beane, P. Bedaque, A. Parreno, and M. Savage, Exploring hyperons and hypernuclei with lattice QCD, Nucl.Phys. A747 (2005) 55-74, [nucl-th/0311027].

[151] S. R. Beane, P. Bedaque, A. Parreno, and M. Savage, Two nucleons on a lattice, Phys.Lett. B585 (2004) 106-114, hep-lat/0312004.

[152] X. Li and C. Liu, Two particle states in an asymmetric box, Phys.Lett. B587 (2004) 100-104, hep-lat/0311035.

[153] W. Detmold and M. J. Savage, Electroweak matrix elements in the two nucleon sector from lattice QCD, Nucl.Phys. A743 (2004) 170-193, hep-lat/0403005. 
[154] P. F. Bedaque, Aharonov-Bohm effect and nucleon nucleon phase shifts on the lattice, Phys.Lett. B593 (2004) 82-88, nucl-th/0402051].

[155] X. Feng, X. Li, and C. Liu, Two particle states in an asymmetric box and the elastic scattering phases, Phys.Rev. D70 (2004) 014505, hep-lat/0404001.

[156] V. Bernard, M. Lage, U.-G. Meissner, and A. Rusetsky, Resonance properties from the finite-volume energy spectrum, JHEP 0808 (2008) 024, arXiv:0806.4495.

[157] S. Bour, S. Koenig, D. Lee, H.-W. Hammer, and U.-G. Meissner, Topological phases for bound states moving in a finite volume, Phys.Rev. D84 (2011) 091503, arXiv:1107.1272].

[158] M. Gockeler, R. Horsley, M. Lage, U.-G. Meissner, P. Rakow, et al., Scattering phases for meson and baryon resonances on general moving-frame lattices, Phys.Rev. D86 (2012) 094513, arXiv:1206.4141.

[159] N. Ishizuka, Derivation of Luscher's finite size formula for $N$ pi and $N N$ system, PoS LAT2009 (2009) 119, arXiv:0910.2772.

[160] R. A. Briceno, On the Determination of Elastic and Inelastic Nuclear Observables from Lattice QCD, arXiv:1311.6032.

[161] R. A. Briceno, Z. Davoudi, and T. C. Luu, Two-Nucleon Systems in a Finite Volume: (I) Quantization Conditions, Phys.Rev. D88 (2013) 034502, arXiv:1305.4903.

[162] R. A. Briceno, Z. Davoudi, T. Luu, and M. J. Savage, Two-Nucleon Systems in a Finite Volume: (II) 3S1-3D1 Coupled Channels and the Deuteron, arXiv:1309.3556.

[163] R. A. Briceno, Z. Davoudi, T. C. Luu, and M. J. Savage, Two-Baryon Systems with Twisted Boundary Conditions, arXiv:1311.7686.

[164] N. Li and C. Liu, Generalized Lúscher Formula in Multi-channel Baryon-Meson Scattering, Phys.Rev. D87 (2013) 014502, arXiv:1209.2201.

[165] P. Guo, J. Dudek, R. Edwards, and A. P. Szczepaniak, Coupled-channel scattering on a torus, Phys.Rev. D88 (2013) 014501, arXiv:1211.0929|.

[166] N. Li, S.-Y. Li, and C. Liu, Generalized Lüscher's Formula in Multichannel Baryon-Baryon Scattering, arXiv:1401.5569.

[167] R. A. Briceno, Two-particle multichannel systems in a finite volume with arbitrary spin, arXiv:1401.3312. 
[168] M. T. Hansen and S. R. Sharpe, Relativistic, model-independent, three-particle quantization condition, arXiv:1311.4848.

[169] CLQCD Collaboration Collaboration, X. Li et al., Anisotropic lattice calculation of pion scattering using an asymmetric box, JHEP 0706 (2007) 053, hep-lat/0703015.

[170] S. Durr, Z. Fodor, J. Frison, C. Hoelbling, R. Hoffmann, et al., Ab-Initio Determination of Light Hadron Masses, Science 322 (2008) 1224-1227, arXiv:0906.3599.

[171] NPLQCD Collaboration Collaboration, S. R. Beane et al., Evidence for a Bound H-dibaryon from Lattice QCD, Phys.Rev.Lett. 106 (2011) 162001, arXiv:1012.3812.

[172] S. R. Beane, E. Chang, W. Detmold, B. Joo, H. Lin, et al., Present Constraints on the H-dibaryon at the Physical Point from Lattice QCD, Mod.Phys.Lett. A26 (2011) 2587-2595, arXiv:1103.2821.

[173] S. R. Beane, E. Chang, S. D. Cohen, W. Detmold, H. Lin, et al., Light Nuclei and Hypernuclei from Quantum Chromodynamics in the Limit of SU(3) Flavor Symmetry, Phys.Rev. D87 (2013) 034506, arXiv:1206.5219.

[174] S. Beane, E. Chang, S. Cohen, W. Detmold, H.-W. Lin, et al., Hyperon-Nucleon Interactions and the Composition of Dense Nuclear Matter from Quantum Chromodynamics, Phys.Rev.Lett. 109 (2012) 172001, arXiv:1204.3606.

[175] T. Yamazaki, K.-i. Ishikawa, Y. Kuramashi, and A. Ukawa, Helium nuclei, deuteron and dineutron in 2+1 flavor lattice QCD, Phys.Rev. D86 (2012) 074514, arXiv:1207.4277.

[176] NPLQCD Collaboration Collaboration, S. R. Beane et al., The Deuteron and Exotic Two-Body Bound States from Lattice QCD, Phys.Rev. D85 (2012) 054511, arXiv:1109.2889.

[177] S. Beane, E. Chang, S. Cohen, W. Detmold, P. Junnarkar, et al., Nucleon-Nucleon Scattering Parameters in the Limit of SU(3) Flavor Symmetry, Phys.Rev. C88 (2013) 024003, arXiv:1301.5790.

[178] NPLQCD Collaboration Collaboration, S. R. Beane et al., The I=2 pipi S-wave Scattering Phase Shift from Lattice QCD, Phys.Rev. D85 (2012) 034505, arXiv:1107.5023.

[179] C. S. Pelissier, A. Alexandru, and F. X. Lee, $\rho$ meson decay on asymmetrical lattices, PoS LATTICE2011 (2011) 134, arXiv:1111.2314. 
[180] CP-PACS Collaboration Collaboration, S. Aoki et al., Lattice QCD Calculation of the rho Meson Decay Width, Phys.Rev. D76 (2007) 094506, arXiv:0708.3705.

[181] C. Lang, D. Mohler, S. Prelovsek, and M. Vidmar, Coupled channel analysis of the rho meson decay in lattice QCD, Phys.Rev. D84 (2011) 054503, arXiv:1105.5636.

[182] C. Pelissier and A. Alexandru, Resonance parameters of the rho-meson from asymmetrical lattices, Phys.Rev. D87 (2013) 014503, arXiv:1211.0092.

[183] S. Ozaki and S. Sasaki, Lúscher's finite size method with twisted boundary conditions: an application to $J / \psi-\phi$ system to search for narrow resonance, Phys.Rev. D87 (2013) 014506, arXiv:1211.5512.

[184] M. I. Buchoff, T. C. Luu, and J. Wasem, S-wave scattering of strangeness -3 baryons, Phys.Rev. D85 (2012) 094511, arXiv:1201.3596].

[185] J. J. Dudek, R. G. Edwards, and C. E. Thomas, Energy dependence of the $\rho$ resonance in $\pi \pi$ elastic scattering from lattice QCD, Phys.Rev. D87 (2013) 034505, arXiv:1212.0830.

[186] J. J. Dudek, R. G. Edwards, and C. E. Thomas, S and D-wave phase shifts in isospin-2 pi pi scattering from lattice QCD, Phys.Rev. D86 (2012) 034031, arXiv:1203.6041.

[187] D. Mohler, C. Lang, L. Leskovec, S. Prelovsek, and R. Woloshyn, $D_{s 0}^{*}(2317)$ Meson and D-Meson-Kaon Scattering from Lattice QCD, Phys.Rev.Lett. 111 (2013) 222001, arXiv:1308.3175.

[188] C. Lang, L. Leskovec, D. Mohler, and S. Prelovsek, Axial resonances a1(1260), b1(1235) and their decays from the lattice, arXiv:1401.2088.

[189] P. Guo, Coupled-channel scattering in $1+1$ dimensional lattice model, Phys.Rev. D88 (2013), no. 1 014507, arXiv:1304.7812.

[190] C. D. Lin, G. Martinelli, C. T. Sachrajda, and M. Testa, $K \rightarrow \pi \pi$ decays in a finite volume, Nucl.Phys. B619 (2001) 467-498, hep-lat/0104006.

[191] H. B. Meyer, Lattice QCD and the two-photon decay of the neutral pion, Eur.Phys.J. A49 (2013) 84, arXiv:1303.0138.

[192] V. Bernard, D. Hoja, U.-G. Meissner, and A. Rusetsky, Matrix elements of unstable states, JHEP 1209 (2012) 023, arXiv:1205.4642. 
[193] T. Blum, P. Boyle, N. Christ, N. Garron, E. Goode, et al., K to $\pi \pi$ Decay amplitudes from Lattice QCD, Phys.Rev. D84 (2011) 114503, arXiv:1106.2714.

[194] T. Blum, P. Boyle, N. Christ, N. Garron, E. Goode, et al., The $K \rightarrow(\pi \pi)_{I=2}$ Decay Amplitude from Lattice QCD, Phys.Rev.Lett. 108 (2012) 141601, arXiv:1111.1699.

[195] T. Blum, P. Boyle, N. Christ, N. Garron, E. Goode, et al., Lattice determination of the $K \rightarrow(\pi \pi)_{I=2}$ Decay Amplitude $A_{2}$, Phys.Rev. D86 (2012) 074513, arXiv:1206.5142.

[196] M. Ciuchini, E. Franco, G. Martinelli, and L. Silvestrini, Two-body nonleptonic decays on the lattice, Phys.Lett. B380 (1996) 353-362, hep-ph/9604240.

[197] M. Testa, Nonperturbative renormalization and kaon physics, Nucl.Phys.Proc.Suppl. 63 (1998) 38-46, hep-lat/9709044.

[198] C. Dawson, G. Martinelli, G. Rossi, C. T. Sachrajda, S. R. Sharpe, et al., New lattice approaches to the delta $I=1 / 2$ rule, Nucl.Phys. B514 (1998) 313-335, hep-lat/9707009.

[199] M. F. Golterman and K. C. Leung, Chiral perturbation theory for $K^{+} \rightarrow \pi^{+} \pi^{0}$ decay in the continuum and on the lattice, Phys.Rev. D56 (1997) 2950-2969, hep-lat/9702015.

[200] G. Rossi, Lattice approach to the Delta $I=1 / 2$ rule, hep-lat/9811009.

[201] A. Buras, M. Ciuchini, E. Franco, G. Isidori, G. Martinelli, et al., Final state interactions and epsilon-prime / epsilon: A Critical look, Phys.Lett. B480 (2000) 80-86, hep-ph/0002116.

[202] E. Pallante, Final state interactions and epsilon-prime / epsilon, Nucl.Phys.Proc.Suppl. 96 (2001) 336-341, hep-ph/0010011.

[203] G. Colangelo, Chiral perturbation theory, dispersion relations and final state interactions in $K \rightarrow \pi \pi$, Nucl.Phys.Proc.Suppl. 106 (2002) 53-61, hep-lat/0111003.

[204] C. D. Lin, G. Martinelli, C. Sachrajda, and M. Testa, Finite volume effects in weak hadronic decays, Nucl.Phys.Proc.Suppl. 109A (2002) 218-225, hep-lat/0111033.

[205] J. Laiho and A. Soni, On lattice extraction of $K \rightarrow \pi \pi$ amplitudes to $O\left(p^{* *} 4\right)$ in chiral perturbation theory, Phys.Rev. D65 (2002) 114020, hep-ph/0203106. 
[206] JLQCD Collaboration Collaboration, S. Aoki et al., $K^{+} \rightarrow \pi^{+} \pi^{0}$ decay amplitude with the Wilson quark action in quenched lattice QCD, Phys.Rev. D58 (1998) 054503, hep-lat/9711046.

[207] J. Bijnens, E. Pallante, and J. Prades, Obtaining $K \rightarrow \pi \pi$ from off-shell $K \rightarrow \pi$ amplitudes, Nucl.Phys. B521 (1998) 305-333, hep-ph/9801326.

[208] D. Pekurovsky and G. Kilcup, Matrix elements relevant for Delta $I=1 / 2$ rule and epsilon-prime / epsilon from lattice QCD with staggered fermions, Phys.Rev. D64 (2001) 074502, hep-lat/9812019.

[209] D. Pekurovsky and G. Kilcup, Lattice calculation of matrix elements relevant for Delta $I=1 / 2$ rule and epsilon-prime / epsilon, hep-lat/9903025.

[210] D. Pekurovsky, Calculation of hadronic matrix elements relevant for delta $I=1 / 2$ rule and epsilon-prime / epsilon in lattice QCD with staggered fermions, hep-lat/9909141.

[211] RBC Collaboration Collaboration, R. D. Mawhinney, $K \rightarrow \pi \pi$ decays with domain wall fermions: Towards physical results, Nucl.Phys.Proc.Suppl. 94 (2001) 315-318, hep-lat/0010030.

[212] S. Capitani and L. Giusti, Analysis of the Delta $I=1 / 2$ rule and epsilon-prime / epsilon with overlap fermions, Phys.Rev. D64 (2001) 014506, hep-lat/0011070.

[213] C.-h. Kim and N. H. Christ, $K \rightarrow \pi \pi$ decay amplitudes from the lattice, Nucl.Phys.Proc.Suppl. 119 (2003) 365-367, hep-lat/0210003.

[214] J. Laiho and A. Soni, Lattice extraction of $K \rightarrow \pi \pi$ amplitudes to NLO in partially quenched and in full chiral perturbation theory, Phys.Rev. D71 (2005) 014021, hep-lat/0306035.

[215] RIKEN-BNL-Columbia Collaboration Collaboration, T. Yamazaki, Calculation of Delta $I=3 / 2$ kaon weak matrix elements including two-pion interaction effects in finite volume, PoS LAT2006 (2006) 100, hep-lat/0610051.

[216] RBC Collaboration, UKQCD Collaboration Collaboration, T. Yamazaki, On-shell Delta I = 3/2 kaon weak matrix elements with non-zero total momentum, Phys.Rev. D79 (2009) 094506, arXiv:0807.3130.

[217] N. Byers and C. N. Yang, Theoretical considerations concerning quantized magnetic flux in superconducting cylinders, Phys. Rev. Lett. 7 (Jul, 1961) 46-49. 
[218] R. Johnson, ANGULAR MOMENTUM ON A LATTICE, Phys.Lett. B114 (1982) 147.

[219] D. C. Moore and G. T. Fleming, Angular momentum on the lattice: The Case of non-zero linear momentum, Phys.Rev. D73 (2006) 014504, hep-lat/0507018.

[220] Lattice Hadron Physics Collaboration (LHPC) Collaboration, S. Basak et al., Clebsch-Gordan construction of lattice interpolating fields for excited baryons, Phys.Rev. D72 (2005) 074501, hep-lat/0508018.

[221] D. C. Moore and G. T. Fleming, Multiparticle States and the Hadron Spectrum on the Lattice, Phys.Rev. D74 (2006) 054504, hep-lat/0607004].

[222] J. J. Dudek, R. G. Edwards, M. J. Peardon, D. G. Richards, and C. E. Thomas, Highly excited and exotic meson spectrum from dynamical lattice QCD, Phys.Rev.Lett. 103 (2009) 262001, arXiv:0909.0200].

[223] J. J. Dudek, R. G. Edwards, M. J. Peardon, D. G. Richards, and C. E. Thomas, Toward the excited meson spectrum of dynamical QCD, Phys.Rev. D82 (2010) 034508, arXiv:1004.4930.

[224] T. Luu and M. J. Savage, Extracting Scattering Phase-Shifts in Higher Partial-Waves from Lattice QCD Calculations, Phys.Rev. D83 (2011) 114508, arXiv:1101.3347.

[225] R. G. Edwards, J. J. Dudek, D. G. Richards, and S. J. Wallace, Excited state baryon spectroscopy from lattice QCD, Phys.Rev. D84 (2011) 074508, arXiv:1104.5152.

[226] C. E. Thomas, R. G. Edwards, and J. J. Dudek, Helicity operators for mesons in flight on the lattice, Phys.Rev. D85 (2012) 014507, [arXiv:1107.1930].

[227] J. E. Drut and A. N. Nicholson, Lattice methods for strongly interacting many-body systems, J.Phys. G40 (2013) 043101, arXiv:1208.6556.

[228] M. G. Endres, D. B. Kaplan, J.-W. Lee, and A. N. Nicholson, Lattice Monte Carlo calculations for unitary fermions in a finite box, Phys.Rev. A87 (2013), no. 2 023615, arXiv:1203.3169.

[229] P. F. Bedaque, I. Sato, and A. Walker-Loud, Finite volume corrections to pi-pi scattering, Phys.Rev. D73 (2006) 074501, hep-lat/0601033.

[230] I. Sato and P. F. Bedaque, Fitting two nucleons inside a box: Exponentially suppressed corrections to the Luscher's formula, Phys.Rev. D76 (2007) 034502, hep-lat/0702021. 
[231] H.-X. Chen and E. Oset, The pion-pion Interaction in the rho Channel in Finite Volume, Phys.Rev. D87 (2013) 016014, arXiv:1202.2787].

[232] M. Albaladejo, G. Rios, J. Oller, and L. Roca, Finite volume treatment of $\pi \pi$ scattering in the $\rho$ channel, arXiv:1307.5169.

[233] R. R. Horgan, Z. Liu, S. Meinel, and M. Wingate, Lattice QCD calculation of form factors describing the rare decays $B \rightarrow K^{*} \ell^{+} \ell^{-}$and $B_{s} \rightarrow \phi \ell^{+} \ell^{-}$, Phys.Rev. D89 (2014) 094501, arXiv:1310.3722.

[234] R. R. Horgan, Z. Liu, S. Meinel, and M. Wingate, Calculation of $B^{0} \rightarrow K^{* 0} \mu^{+} \mu^{-}$ and $B_{s}^{0} \rightarrow \phi \mu^{+} \mu^{-}$observables using form factors from lattice QCD, Phys.Rev.Lett. 112 (2014) 212003, arXiv:1310.3887.

[235] S. Prelovsek, Lattice QCD review of charmonium and open-charm spectroscopy, arXiv:1310.4354,

[236] C. Thomas, Hadron Spectroscopy Review, PoS LATTICE2013 (2014) 003.

[237] M. Doering, Resonances and multi-particle states, PoS LATTICE2013 (2014) 006.

[238] P. Guo, The three-particle system on a torus, arXiv:1303.3349.

[239] S. Aoki, N. Ishii, T. Doi, Y. Ikeda, and T. Inoue, Asymptotic behavior of Nambu-Bethe-Salpeter wave functions for multiparticles in quantum field theories, Phys.Rev. D88 (2013), no. 1 014036, [arXiv:1303.2210].

[240] M. Rubin, R. Sugar, and G. Tiktopoulos, Dispersion Relations for Three-Particle Scattering Amplitudes. I, Phys.Rev. 146 (1966) 1130-1149.

[241] D. Brayshaw, Off- and on-shell analyticity of three-particle scattering amplitudes, Phys.Rev. 176 (1968) 1855-1870.

[242] V. Potapov and J. Taylor, Three-particle scattering rates and singularities of the T matrix. I., Phys.Rev. A16 (1977) 2264-2275.

[243] V. Potapov and J. Taylor, Three-particle scattering rates and singularities of the T matrix. II., Phys.Rev. A16 (1977) 2276-2287.

[244] S. Sasaki and T. Yamazaki, Signatures of S-wave bound-state formation in finite volume, Phys.Rev. D74 (2006) 114507, hep-lat/0610081.

[245] S. R. Beane, W. Detmold, and M. J. Savage, n-Boson Energies at Finite Volume and Three-Boson Interactions, Phys.Rev. D76 (2007) 074507, arXiv:0707.1670. 
[246] S. Tan, Three-boson problem at low energy and Implications for dilute Bose-Einstein condensates, Phys.Rev. A78 (2008) 013636, arXiv:0709.2530.

[247] G. Sterman, An Introduction to Quantum Field Theory. Cambridge University Press, 9, 1993.

[248] S. Weinberg, The Quantum theory of fields. Vol. 1: Foundations, .

[249] P. F. Bedaque and J.-W. Chen, Twisted valence quarks and hadron interactions on the lattice, Phys.Lett. B616 (2005) 208-214, hep-lat/0412023.

[250] D. Agadjanov, U.-G. Meissner, and A. Rusetsky, Partially twisted boundary conditions for scalar mesons, arXiv:1310.7875

[251] J. E. Mandula, G. Zweig, and J. Govaerts, Representations of the Rotation Reflection Symmetry Group of the Four-dimensional Cubic Lattice, Nucl.Phys. B228 (1983) 91. 


\section{Appendix A}

\section{TWO-CHANNEL WATSON'S THEOREM}

In this appendix we work out the consequences of Watson's theorem for the phases of the matrix elements of interest, $\left\langle\pi \pi\left|\mathcal{H}_{W}(0)\right| D\right\rangle$ and $\left\langle K \bar{K}\left|\mathcal{H}_{W}(0)\right| D\right\rangle$. We assume at first that $\mathcal{H}_{W}$ is $\mathrm{T}$ invariant, and describe the generalization to non-invariant Hamiltonians at the end. We closely follow the textbook presentation given in Ref. [248].

We consider the $3 \times 3 S$-matrix with the three states being the hypothetical $D$ meson (at rest) and the s-wave $\pi \pi$ and $K \bar{K}$ states. We assume that we are in the kinematic regime described in the main text, so that the $3 \times 3 S$-matrix is unitary. Although we introduce a weak coupling between the $D$ and the two particle states, so that the $D$ is a resonance, its width is of second-order in the weak interaction and thus can be ignored at the linear order to which we work. Thus it is valid to treat it as an asymptotic state.

Watson's theorem follows by breaking the $S$-matrix into a strong part $S^{(0)}$ and a weak part $S^{W}$. The strong part is $\mathrm{T}$ invariant, and, since we use states which have definite (positive) T-parity, can be taken to be symmetric. This fixes the phases of the $\pi \pi$ and $K \bar{K}$ states, though not their overall signs. Extending the dimensionless, strong-coupling $S$-matrix of Eq. 2.47) to include the $D$ gives

$$
S^{(0)}=\left(\begin{array}{cc}
1 & 0 \\
0 & S^{s}
\end{array}\right),
$$

where 1 is the $1 \times 1$ identity and $S^{s}$ is the $2 \times 2$ s-wave $S$-matrix given in (2.47). The weak part only contains couplings between the $D$ and the two-particle states, and in $3 \times 3$ 
notation is

$$
S^{W}=\left(\begin{array}{ccc}
0 & S_{D, \pi \pi}^{W} & S_{D, K K}^{W} \\
S_{\pi \pi, D}^{W} & 0 & 0 \\
S_{K K, D}^{W} & 0 & 0
\end{array}\right)
$$

The assumed T invariance implies that it, too, is symmetric. The non-zero elements of $S^{W}$ are proportional to the desired matrix elements

$$
S_{j, D}^{W}=c P_{j j}\left\langle j\left|\left[-i \mathcal{H}_{W}(0)\right]\right| D\right\rangle,
$$

where $j=1,2$ runs over the $\pi \pi$ and $K \bar{K}$ channels, $P$ is the square root of the phase space factor defined in Eq. (2.46), and $c$ is a known real constant whose value will not be needed.

Unitarity of the complete $S$-matrix implies that the terms linear in the weak interaction satisfy

$$
i S^{W}=S^{(0)}\left(i S^{W}\right)^{\dagger} S^{(0)}
$$

This implies that

$$
i S_{j, D}^{W}=S_{j k}^{s}\left(i S_{D, k}^{W}\right)^{*}=S_{j k}^{s}\left(i S_{k, D}^{W}\right)^{*}
$$

where in the last step we have used the symmetry of $S^{W}$. Using the explicit form for the two-channel $S$-matrix 1

$$
S^{s}=R^{-1}\left(\begin{array}{cc}
e^{2 i \delta_{1}} & 0 \\
0 & e^{2 i \delta_{2}}
\end{array}\right) R
$$

with

$$
R=\left(\begin{array}{cc} 
& \\
\mathrm{c}_{\epsilon} & \mathrm{s}_{\epsilon} \\
-\mathrm{s}_{\epsilon} & \mathrm{c}_{\epsilon}
\end{array}\right)
$$

we find

$$
i R_{j k} S_{k, D}^{W}=e^{2 i \delta_{j}}\left(i R_{j k} S_{k, D}^{W}\right)^{*}
$$

\footnotetext{
${ }^{1}$ For simplicity of presentation, we are here using $\delta_{1}=\delta_{\alpha}$ and $\delta_{2}=\delta_{\beta}$.
} 
It follows that the phase of $i R_{j k} S_{k, D}^{W}$ is $e^{i \delta_{j}}$. This is the desired generalization of Watson's theorem to two channels. Thus the quantities

$$
v_{j}=e^{-i \delta_{j}} \frac{1}{c} \sqrt{4 \pi E^{*}} i R_{j k} S_{k, D}^{W}
$$

are real. Using (A.3) we can rewrite the $v_{j}$ as in Eqs. 3.15) and (3.16).

If the weak interaction is not $\mathrm{T}$ invariant, then $S_{j, D}^{W}$ will contain some number of $\mathrm{T}$ violating phases. Since we are working to linear order in the weak interaction, we can break up $\mathcal{H}_{W}$ into parts each with a single T-violating phase and treat each separately. Each such part has an overall phase $e^{i \phi_{T}}$, and the symmetry of the S-matrix is replaced by

$$
S_{D, k}^{W}\left(\phi_{T}\right)=S_{k, D}^{W}\left(-\phi_{T}\right)
$$

However, if we first pull out the overall phase by hand, then the symmetry of $\Delta S$ is restored, and Watson's theorem applies to the residue. 


\section{Appendix B}

\section{CANCELATION OF FREE POLES}

At arriving at the final expression for the two-point correlation, Eq. 4.55, we argued that the free particle poles of the integrand of Eq. 4.43 do not contribute, and here we give a proof of this statement. In Sections $4.1 \& 4.2$, we constructed operators that are in the irrep of the symmetry group of the system, but the cancelation of free poles cannot depend on this fact. It must only depend on the fact that the particles interactions are not exactly zero. If one would choose to not properly define an operator with good quantum numbers, then Eq. 4.19 would acquire an additional sum over all possible irreps that have overlap with the operator of interest. This in turn would lead to a far less reliable extraction of the spectrum since multiple irreps could in principle have nearly degenerate eigenstates. With this caveat in mind, we decide to illustrate the cancelation of free particle poles using a set of generic operators with no particular set of quantum numbers

$$
\begin{gathered}
\mathcal{A}\left(x_{0}, \mathbf{P}\right)=\sum_{\mathbf{k}} a(\mathbf{k}) \varphi\left(x_{0}, \mathbf{P}-\mathbf{k}\right) \widetilde{\varphi}\left(x_{0}, \mathbf{k}\right), \\
\mathcal{B}\left(x_{0}, \mathbf{P}\right)=\sum_{\mathbf{k}} b(\mathbf{k}) \varphi^{\dagger}\left(x_{0},-\mathbf{P}+\mathbf{k}\right) \widetilde{\varphi}^{\dagger}\left(x_{0},-\mathbf{k}\right),
\end{gathered}
$$

where $a(\mathbf{k})$ and $b(\mathbf{k})$ are some generic function of $\mathbf{k}$. Note that we have not specified wether the sum is over all possible values of $\mathbf{k}$ or one specific shell; this distinction does not matter. Additionally, we restrict the discussion to where there is only one channel open, since this observation is independent of the number of open channels. Using this notation, it is straightforward to write down the two-point correlation function in the vicinity of the 
free poles

$$
\begin{aligned}
& \left\langle 0\left|\mathcal{A}\left(x_{0}, \mathbf{P}\right) \mathcal{B}\left(y_{0}, \mathbf{P}\right)\right| 0\right\rangle=L^{3} \int \frac{d P_{0}}{2 \pi} e^{i P_{0}\left(x_{0}-y_{0}\right)}\left[\sum_{\mathbf{k}} \frac{-i L^{3}}{4 \omega_{1, \mathbf{P}-\mathbf{k}} \omega_{2, \mathbf{k}}} \frac{a(\mathbf{k}) b(\mathbf{k})}{P_{0}-i\left(\omega_{1, \mathbf{P}-\mathbf{k}}+\omega_{2, \mathbf{k}}\right)}\right. \\
& \left.-\sum_{\mathbf{k}, \mathbf{k}^{\prime}} \frac{a(\mathbf{k}) b\left(\mathbf{k}^{\prime}\right) \mathbb{T}_{L}\left(P, k, k^{\prime}\right)}{4 \omega_{1, \mathbf{P}-\mathbf{k}} \omega_{2, \mathbf{k}}\left[P_{0}-i\left(\omega_{1, \mathbf{P}-\mathbf{k}}+\omega_{2, \mathbf{k}}\right)\right] 4 \omega_{1, \mathbf{P}-\mathbf{k}^{\prime}} \omega_{2, \mathbf{k}^{\prime}}\left[P_{0}-i\left(\omega_{1, \mathbf{P}-\mathbf{k}^{\prime}}+\omega_{2, \mathbf{k}^{\prime}}\right)\right]}+\cdots\right],
\end{aligned}
$$

where the ellipses denote finite contributions to the correlation function near the free poles. The correlation function is illustrated in Fig. B. In writing the correlation function we have introduce a function $\mathbb{T}_{L}\left(P, k, k^{\prime}\right)$, which is related to the K-matrix via Eq. 4.38 , Near the free particle poles this can be written as

$$
\mathbb{T}_{L}\left(P, k, k^{\prime}\right)=-\mathbb{K}\left(P, k, k^{\prime}\right)+i\left[\frac{1}{L^{3}} \sum_{\mathbf{l}}-\int_{\mathbf{l}}\right] \frac{\mathbb{K}(P, k, l) \mathbb{T}_{L}\left(P, l, k^{\prime}\right)}{4 \omega_{1, \mathbf{P}-\mathbf{l}} \omega_{2, \mathbf{l}}(\mathbf{l})\left[P_{0}-i\left(\omega_{1, \mathbf{P}-\mathbf{l}}+\omega_{2, \mathbf{l}}\right)\right]},
$$

where we have neglected contributions suppressed at the free two-particle poles. The free particle poles satisfy $P_{0}=i\left(\omega_{1, \mathbf{P}-\mathbf{k}}+\omega_{2, \mathbf{k}}\right)$ and in order to obtain the contribution of these, we investigate the leading $\epsilon$ behavior, where $\epsilon$ is defined via

$$
P_{0}=i\left(\omega_{1, \mathbf{P}-\mathbf{k}}+\omega_{2, \mathbf{k}}\right)+\epsilon
$$

To do so, we again upgrade these functions to be matrices in momentum space. It is important to observe that in general, there will be multiple values of $\mathbf{k}$ and $\mathbf{P}-\mathbf{k}$ that will satisfy the free energy condition, these are of course the $\{\mathbf{k}\}^{\mathbf{P}}$ and $\{\mathbf{P}-\mathbf{k}\}^{\mathbf{P}}$. By defining $\omega_{1}$ and $\omega_{2}$ as the free energies that satisfy $P_{0}=i\left(\omega_{1}+\omega_{2}\right)$, at leading order in $\epsilon$, Eq. B.3 simplifies to

$$
-i L^{3} a\left[\frac{1}{4 \omega_{1} \omega_{2} \epsilon}\right]^{\star} b-a\left[\frac{1}{4 \omega_{1} \omega_{2} \epsilon}\right]^{\star} \mathbb{T}_{L}\left[\frac{1}{4 \omega_{1} \omega_{2} \epsilon}\right]^{\star} b
$$

Here $a$ is understood as a row and $b$ as a column vector, $\left[1 /\left(4 \omega_{1} \omega_{2} \epsilon\right)\right]^{\star}$ is a diagonal matrix that is zero if the value of the momenta are not in $\{\mathbf{k}\}^{\mathbf{P}}$ and $\{\mathbf{P}-\mathbf{k}\}^{\mathbf{P}}$ and equal to $1 /\left(4 \omega_{1} \omega_{2} \epsilon\right)$ otherwise, while $\mathbb{T}_{L}$ is a matrix with off-diagonal entries. By restricting ourselves to the set of momenta that satisfy the free energy conditions, the $T$-matrix, Eq. B.4, satisfies

$$
\mathbb{T}_{L}=-\mathbb{K}+i \frac{1}{L^{3}} \mathbb{K}\left[\frac{1}{4 \omega_{1} \omega_{2} \epsilon}\right]^{\star} \mathbb{T}_{L}
$$


At this stage we observe that, since $\mathbb{K}=\mathcal{O}(1)$, one can shown that

$$
\mathbb{T}_{L}=-i L^{3}\left[\frac{1}{4 \omega_{1} \omega_{2} \epsilon}\right]^{-1}+\mathcal{O}\left(\epsilon^{2}\right) .
$$

Substituting this into Eq. B.6 gives perfect cancellation of the $\mathcal{O}(1 / \epsilon)$ terms independent of the values of $a$ and $b$. This justifies the cancellation of free particle poles in Eq. 4.43, which is recovered by setting $a$ and $b$ equal to the Clebsch-Gordan coefficients.

However, it is common practice to restrict the scattering amplitude to a particular partial wave when obtaining the finite volume spectrum. Here we demonstrate how this approximation can lead to spurious free poles in the correlation function. Let $\mathbb{K}_{S}\left(n, P_{0}\right)$ and $\mathbb{T}_{S}\left(n, P_{0}\right)$ be the S-wave K-matrix and $T$ functions at the $n t h$ free particle pole which has a degeneracy of $N$. From Eq. B.4, we see that these satisfy

$$
\begin{aligned}
\mathbb{T}_{S}\left(n, P_{0}\right) & =-\mathbb{K}_{S}\left(n, P_{0}\right)+i \frac{N}{L^{3}} \frac{\mathbb{K}_{S}\left(n, P_{0}\right) \mathbb{T}_{S}\left(n, P_{0}\right)}{4 \omega_{1} \omega_{2} \epsilon}+\mathcal{O}\left(\epsilon^{2}\right), \\
\Rightarrow \mathbb{T}_{S}\left(n, P_{0}\right) & =-i \frac{4 \omega_{1} \omega_{2} L^{3}}{N} \epsilon+\mathcal{O}\left(\epsilon^{2}\right) .
\end{aligned}
$$

Substituting Eq. B.10 into the s-wave reduction of Eq. B.3, we deduce that free particle poles only cancel when

$$
(1 / N) \sum_{R, R^{\prime} \in \mathrm{LG}(\mathbf{P})} a(R \mathbf{k}) b\left(R \mathbf{k}^{\prime}\right)=\sum_{R \in \mathrm{LG}(\mathbf{P})} a(R \mathbf{k}) b(R \mathbf{k})
$$

If one chooses $a$ and $b$ to be Kronecker deltas in momentum, as is done in Ref. [192], the cancellation in Eq. B.3 is lost, unless $N=1$. But this is a contradiction to the statement above, that free particle poles should not appear regardless of the values of $a$ and $b$ for any momentum. The apparent contradiction here is resolved by noting that the matrix $\mathbb{K}$ is only invertible if each row is linearly independent. However, in the case of s-wave amplitude the matrix is proportional to a matrix which has 1 in every single entry. Thus the matrix argument fails and the alternative argument shows that cancellation does not occur for all $a$ and $b$. Furthermore, we argue that imposing a scattering amplitude to exactly vanish for all but one partial waves at all values of momentum is unnatural. The only way to achieve this is to require all shape parameters of the partial waves not included to be equal to zero. Restricting the final results of quantization condition, the matrix elements of the 


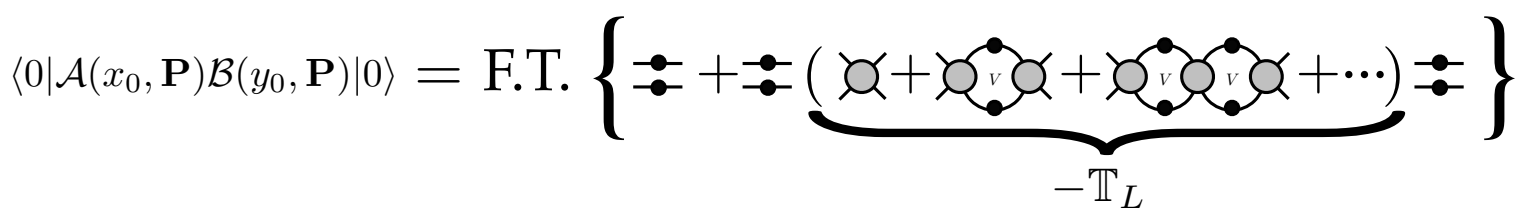

Figure B.1: Shown is the diagrammatic representation of the correlation function defined in Eq. B.3 in terms of the kernels (defined in Fig. 4.1(b)], the fully dressed single particle propagators (defined in Fig. 4.1(c) and the finite volume loops. The "F. T." label around the braces reminds the reader that one must Fourier transform the energy-momentum correlation function to obtain the correct exponential dependence in time. The $T$ function, which is explicitly labeled, is defined in Eq. 4.38 .

two-particle interpolating operator and the matrix elements of the currents, Eqs. 4.1, 4.57, 4.58, $4.92 \& 4.102$, to a single partial wave can be done if the contribution from higher partial waves is seen to be significantly suppressed at low energies. This is to say that the order of operations in studying finite volume physics is relevant and can lead to significantly different results.

From this discussion it is clear that if one is solely interested in obtaining the spectrum and is not in arriving at a nonperturbative expression for the correlation functions, it suffices to look at the poles of $\mathbb{T}_{L}$. As is evident from Fig. $B$, the free particle poles correspond to zeros of $\mathbb{T}_{L}$, and consequently one does not need to worry about any spurious poles. Furthermore, the subtlety regarding the order of operations does not play a role when studying the pole structure of $\mathbb{T}_{L}$. Therefore, as was done in Ref. [151], one may first proceed to set the angular momentum to any partial wave desired and then obtain the quantization condition from the pole structure of $\mathbb{T}_{L}$. 


\section{Appendix C}

\section{GENERALIZATION FOR TWISTED BOUNDARY CONDITIONS IN ASYMMETRIC VOLUMES}

In the derivation of the master equations of this work, namely Eqs. 4.1, 4.57, 4.58, $4.92 \& 4.102$, periodic boundary condition on the spatial extent of the cubic volume have been assumed. The periodicity constraint is encoded in the expression for the $\mathcal{Z}$ functions shown in Eq. 4.32, and this is generally true for arbitrary boundary conditions, and Ref. [167] demonstrated how to compactly write the $\mathcal{Z}$ functions in such a way that they accommodate the different geometries and boundary conditions. For relevant work that lead to this result, see Refs. [152, 153, 155, 154, 249, 134, 135, 183, 163, 250]. TBCs require that fields in general satisfy

$$
\psi(\mathbf{x}+\mathbf{n} L)=e^{i \boldsymbol{\theta} \cdot \mathbf{n}} \psi(\mathbf{x})
$$

where $\theta$ is a three-dimensional real angle. Therefore, the free momentum of the $i t h$ in the $j$ th channel will be equal to $\mathbf{p}_{j, i}=\frac{2 \pi \mathbf{n}_{i}}{L}+\frac{\phi_{j, i}}{L}$.

For asymmetric volumes, let $L$ to be the spatial extent of the z-axis and $\eta_{i}$ be defined such that $L_{x}=\eta_{x} L$ and $L_{y}=\eta_{y} L$. Using the notation $\tilde{\chi}=\left(\chi_{x} / \eta_{x}, \chi_{y} / \eta_{y}, \chi_{z}\right)$, one can readily find the most general form of the $c_{l m}$ and $\mathcal{Z}$ functions with arbitrary twist and asymmetric volumes

$$
\begin{aligned}
c_{l m}^{\mathbf{d}, \phi_{j, 1}, \phi_{j, 2}}\left(k^{* 2} ; L ; \eta_{x}, \eta_{y}\right) & =\frac{\sqrt{4 \pi}}{\eta_{x} \eta_{y} \gamma L^{3}}\left(\frac{2 \pi}{L}\right)^{l-2} \times \mathcal{Z}_{l m}^{\mathbf{d}, \phi_{j, 1}, \phi_{j, 2}}\left[1 ;\left(k^{*} L / 2 \pi\right)^{2} ; \eta_{x}, \eta_{y}\right] \\
\mathcal{Z}_{l m}^{\mathbf{d}, \phi_{j, 1}, \phi_{j, 2}}\left[s ; x^{2} ; \eta_{x}, \eta_{y}\right] & =\sum_{\mathbf{r} \in \mathcal{P}_{\mathbf{d} ; \eta_{x}, \eta_{y}}^{\phi_{1}, \phi_{2} ;}} \frac{|\mathbf{r}|^{l} Y_{l, m}(\mathbf{r})}{\left(\mathbf{r}^{2}-x^{2}\right)^{s}}
\end{aligned}
$$

where $\mathcal{P}_{\mathbf{d} ; \eta_{x}, \eta_{y}}^{\phi_{1}, \phi_{2}}=\left\{\mathbf{r} \in \mathbf{R}^{3} \mid \mathbf{r}=\hat{\gamma}^{-1}\left(\widetilde{\mathbf{m}}-\alpha_{j} \widetilde{\mathbf{d}}+\frac{\widetilde{\boldsymbol{\Delta}}^{(j)}}{2 \pi}\right)\right\}$, where $\mathbf{m}$ is a triplet integer, $\widetilde{\boldsymbol{\Delta}}^{(j)}=$ $-\left(\alpha_{j}-\frac{1}{2}\right)\left(\widetilde{\boldsymbol{\phi}}_{j, 1}+\widetilde{\boldsymbol{\phi}}_{j, 2}\right)+\frac{1}{2}\left(\widetilde{\boldsymbol{\phi}}_{j, 1}-\widetilde{\boldsymbol{\phi}}_{j, 2}\right)$ and $\widetilde{\mathbf{d}}=\mathbf{P} L / 2 \pi$. Additionally, one obtained an overall 
factor of $\sqrt{\eta_{x} \eta_{y}}$ in Eqs. $4.9,4.57 \& 4.58$, i.e. one must make the following replacements

$$
\begin{aligned}
\left|\left\langle 0|\varphi(0, \mathbf{k})| E_{k}^{(1)}\right\rangle\right| & \longrightarrow \sqrt{\frac{\eta_{x} \eta_{y} L^{3}}{2 \omega_{k}}} \\
\left|\left\langle 0\left|\mathcal{O}_{\Lambda \mu, a}(0, \mathbf{P})\right| E_{\Lambda, n}\right\rangle\right| & \longrightarrow \sqrt{\eta_{x} \eta_{y} L^{3}} \sqrt{\mathbb{C}_{\Lambda \mu}^{T}\left[\mathbb{Y}_{\Lambda, n} R_{\Lambda, n} \mathbb{Y}_{\Lambda, n}^{\dagger}\right]_{a a} \mathbb{C}_{\Lambda \mu}^{*}} .
\end{aligned}
$$




\section{Appendix D}

\section{SUM-MINUS-INTEGRAL IDENTITY}

In this appendix we derive the sum-minus-integral identity which plays a central role in the main text. This identity is closely related to that given in Ref. [130] in the context of the two particle quantization condition.

The identity is

$$
\frac{1}{2}\left[\frac{1}{L^{3}} \sum_{\vec{a}}-\widetilde{\mathrm{PV}} \int_{\vec{a}}\right] \frac{g(\vec{k}, \vec{a}) h(\vec{k}, \vec{a}) H(\vec{k})}{2 \omega_{a} 2 \omega_{k a}\left(E-\omega_{k}-\omega_{a}-\omega_{k a}\right)}=g_{\ell^{\prime}, m^{\prime}}^{*}(\vec{k}) F_{\ell^{\prime}, m^{\prime} ; \ell, m}(\vec{k}) h_{\ell, m}^{*}(\vec{k}),
$$

which holds up to (implicit) exponentially-suppressed finite-volume corrections. The matrix $F(\vec{k})$ is given in the main text but repeated here for convenience

$$
\begin{aligned}
& F_{\ell^{\prime}, m^{\prime} ; \ell, m}(\vec{k}) \equiv F_{\ell^{\prime}, m^{\prime} ; \ell, m}^{i \epsilon}(\vec{k})+\rho_{\ell^{\prime}, m^{\prime} ; \ell, m}(\vec{k}), \\
& F_{\ell^{\prime}, m^{\prime} ; \ell, m}^{i \epsilon}(\vec{k}) \equiv \frac{1}{2}\left[\frac{1}{L^{3}} \sum_{\vec{a}}-\int_{\vec{a}}\right] \frac{4 \pi Y_{\ell^{\prime}, m^{\prime}}\left(\hat{a}^{*}\right) Y_{\ell, m}^{*}\left(\hat{a}^{*}\right) H(\vec{k}) H(\vec{a}) H\left(\vec{b}_{k a}\right)}{2 \omega_{a} 2 \omega_{k a}\left(E-\omega_{k}-\omega_{a}-\omega_{k a}+i \epsilon\right)}\left(\frac{a^{*}}{q_{k}^{*}}\right)^{\ell+\ell^{\prime}} .
\end{aligned}
$$

The phase-space quantity $\rho$ and the cutoff function $H$ are defined, respectively, in Eqs. 5.25 and (5.28). The kinematic notation is that described in Sec. 5.1. the spectator has fixed four-momentum $\left(\omega_{k}, \vec{k}\right)$, the particle whose momentum is summed/integrated has fourmomentum $\left(\omega_{a}, \vec{a}\right)$, while the third particle is in general off shell, with four-momentum $\left(E_{2}-\omega_{a}, \vec{b}_{k a}\right)$. The four-momentum of the non-spectator pair is $P_{2}=\left(E_{2}, \vec{P}_{2}\right)=$ $\left(E-\omega_{k}, \vec{P}-\vec{k}\right)$, and $\vec{b}_{k a}=\vec{P}_{2}-\vec{a}$. If the third particle were on shell, it would have energy $\omega_{k a}$ [defined in Eq. [5.1]], so the on-shell condition is $E_{2}=\omega_{a}+\omega_{k a}$. This is where the denominator in Eq. D.3 vanishes. The boost to the CM-frame of the non-spectator pair sends $P_{2}$ to $\left(E_{2, k}^{*}, \overrightarrow{0}\right)$ and $\left(\omega_{a}, \vec{a}\right)$ to $\left(\omega_{a}^{*}, \vec{a}^{*}\right)$. If all three particles are on shell, then the magnitude of $\vec{a}^{*}$ satisfies $a^{*}=q_{k}^{*}$ [with $q_{k}^{*}$ given by Eq. (5.6)].

The two functions in the identity (D.1), $g$ and $h$, contain the momentum dependence arising from quantities respectively on the left and right of the three-particle "cut". They could be combined into a single function, but for our formalism it is advantageous to keep 
them separate. We assume that $g$ and $h$ are smooth (infinitely differentiable) functions of the components of $\vec{a}$ and that they fall off at large $|\vec{a}|$ such that the sum and integral are convergent. Note that, since $k$ and $a$ are on-shell, and the total momentum is fixed, the independent quantities are $\vec{k}$ and $\vec{a}$, which are thus given as the arguments of $g$ and $h$. In general, the third momentum is off shell, so these functions involve off-shell amplitudes. What appears on the right-hand side of the identity, however, are on-shell projections of these amplitudes after decomposition into the angular-momentum basis in the CM-frame of the non-spectator pair. This projection is explained around Eq. (5.65) for the case where $g=\sigma$ and $h=\sigma^{\dagger}$, but applies equally well to any functions.

One difference between our identity and that of Ref. [130] is that, in the three-particle context, the two-particle sub-system can be arbitrarily far below threshold. The dominant sub-threshold contribution to $F$ comes from the factor of $\rho$ in Eq. (D.2), which in turn arises from the difference between $\widetilde{\mathrm{PV}}$ and $i \epsilon$ pole prescriptions [see Eq. [5.59]. This factor is needed so that the dependence on $\vec{k}$ is smooth, but is not important for the derivation of the sum-integral identity. Indeed, we can rewrite the identity using the $i \epsilon$-prescription and cancel factors of $\rho$ :

$$
\frac{1}{2}\left[\frac{1}{L^{3}} \sum_{\vec{a}}-\int_{\vec{a}}\right] \frac{g(\vec{k}, \vec{a}) h(\vec{k}, \vec{a}) H(\vec{k})}{2 \omega_{a} 2 \omega_{k a}\left(E_{2}-\omega_{a}-\omega_{k a}+i \epsilon\right)}=g_{\ell^{\prime}, m^{\prime}}^{*}(\vec{k}) F_{\ell^{\prime}, m^{\prime} ; \ell, m}^{i \epsilon}(\vec{k}) h_{\ell, m}^{*}(\vec{k}),
$$

This is now very similar to the identity of Ref. [130], and we focus on this form henceforth.

To demonstrate (D.4) we need simply to subtract the two sides and show that it is exponentially suppressed. The difference is proportional to

$H(\vec{k})\left[\frac{1}{L^{3}} \sum_{\vec{a}}-\int_{\vec{a}}\right] \frac{g(\vec{k}, \vec{a}) h(\vec{k}, \vec{a})-g_{\ell^{\prime}, m^{\prime}}^{*}(\vec{k}) 4 \pi Y_{\ell^{\prime}, m^{\prime}}\left(\hat{a}^{*}\right)\left(a^{*} / q_{k}^{*}\right)^{\ell^{\prime}+\ell} Y_{\ell, m}^{*}\left(\hat{a}^{*}\right) h_{\ell, m}^{*}(\vec{k}) H(\vec{a}) H\left(\vec{b}_{k a}\right)}{2 \omega_{a} 2 \omega_{k a}\left(E_{2}-\omega_{a}-\omega_{k a}+i \epsilon\right)}$,

where we have assumed that the sums over angular-momentum indices can be interchanged with the $\vec{a}$ integral. The overall factor of $H(\vec{k})$ serves only to ensure that the boosts to the two-particle CM frame are well-defined. We note that the sums and integrals are convergent in the ultraviolet because of the assumed properties of $g$ and $h$ (in the first term in the numerator) and the presence of the cutoffs $H$ (in the second term). The difference (D.5) will vanish, up to exponentially suppressed corrections, if the integrand/summand is 
non-singular and smooth as a function of $\vec{a}$. This in turn holds if (i) all functions appearing in the expression have a smooth dependence on $\vec{a}$, and (ii) the difference in the numerator cancels that in the denominator in such a way that the the ratio is smooth. We address these conditions in turn.

The only non-smooth functions appearing in (D.5) are the spherical harmonics, which are ill-defined at $\vec{a}^{*}=0$ for $\ell>0$. Smoothness is ensured, however, by the factors of $\left(a^{*}\right)^{\ell^{\prime}+\ell}$, which turn the spherical harmonics into polynomials in the components of $\vec{a}$. Thus the first condition is satisfied. For subsequent work, it is useful to understand the $a^{*}$ dependence of the coefficients in the angular-momentum expansion of $g$ and $h$. Recall that one first changes to $\vec{a}^{*}$ as the independent variable, e.g. $g^{*}\left(\vec{k}, \vec{a}^{*}\right) \equiv g(\vec{k}, \vec{a})$, and then expands in harmonics:

$$
g^{*}\left(\vec{k}, \vec{a}^{*}\right)=g_{\ell^{\prime}, m^{\prime}}^{*}\left(\vec{k}, a^{*}\right) Y_{\ell^{\prime}, m^{\prime}}\left(\hat{a}^{*}\right) \sqrt{4 \pi}=\frac{g_{\ell^{\prime}, m^{\prime}}^{*}\left(\vec{k}, a^{*}\right)}{a^{* \ell}} Y_{\ell^{\prime}, m^{\prime}}\left(\hat{a}^{*}\right) a^{* \ell} \sqrt{4 \pi} .
$$

For $a^{*} \rightarrow 0$, the last form is simply a rewriting of the Taylor expansion in the spherical basis, since $Y_{\ell^{\prime}, m^{\prime}}\left(\hat{a}^{*}\right) a^{* \ell}$ is a homogeneous polynomial of order $\ell$ in the components of $\vec{a}^{*}$. This implies that $g_{\ell^{\prime}, m^{\prime}}^{*}\left(\vec{k}, a^{*}\right) / a^{* \ell}$ has a finite limit as $a^{*} \rightarrow 0$. Furthermore, since $g^{*}\left(\vec{k}, \vec{a}^{*}\right)$ is, by assumption, smooth at $a^{*}=0, g_{\ell^{\prime}, m^{\prime}}^{*}\left(\vec{k}, a^{*}\right) / a^{* \ell}$ must be a smooth function of $a^{* 2}$ and not $a^{*}$. Thus, for small $a^{*}$,

$$
\frac{g_{\ell^{\prime}, m^{\prime}}^{*}\left(\vec{k}, a^{*}\right)}{a^{* \ell}}=\sum_{n=0}^{\infty} s_{n}\left(a^{* 2}\right)^{n},
$$

with $s_{n}$ the Taylor coefficients. An analogous result holds for $h$.

We turn now to the second condition, that zeroes in the numerator and denominator should cancel. To satisfy this we first need the numerator of (D.5) to vanish on shell. This is true because, when $E_{2}=\omega_{a}+\omega_{k a}$, we have $H(\vec{a})=H\left(\vec{b}_{k a}\right)=1, a^{*}=q_{k}^{*}$, and

$$
g_{\ell^{\prime}, m^{\prime}}^{*}(\vec{k}) Y_{\ell^{\prime}, m^{\prime}}\left(\hat{a}^{*}\right) \sqrt{4 \pi}=g_{\ell^{\prime}, m^{\prime}}^{*}\left(\vec{k}, q_{k}^{*}\right) Y_{\ell^{\prime}, m^{\prime}}\left(\hat{a}^{*}\right) \sqrt{4 \pi}=g^{*}\left(\vec{k}, q_{k}^{*} \hat{a}^{*}\right)=g(\vec{k}, \vec{a})
$$

(and similarly for $h$ ). In addition, the numerator must vanish fast enough to cancel the denominator. To see that this is also true it is convenient to re-express the denominator in terms of CM variables. Following the arguments of Ref. [130], we can make the replacement

$$
\frac{1}{2 \omega_{a} 2 \omega_{k a}\left(E_{2}-\omega_{a}-\omega_{k a}+i \epsilon\right)} \longrightarrow \frac{\omega_{a}^{*}}{2 \omega_{a} E_{2, k}^{*}\left(q_{k}^{*}+a^{*}\right)\left(q_{k}^{*}-a^{*}+i \epsilon\right)},
$$


since the difference is non-singular. This shows us that the singularity lies in the radial integral over $a^{*}$. Now consider the ratio (D.5) at fixed angle $\hat{a}^{*}$, so that the spherical harmonics are fixed. Then, from the discussion above, we know that both terms in the numerator are smooth functions of $a^{*}$, which thus have Taylor expansions about $a^{*}=q_{k}^{*}$. In the difference, the constant term in these Taylor expansions cancels, and so the ratio with $1 /\left(q_{k}^{*}-a *\right)$ is a smooth function of $a^{*}$. In particular we have demonstrated that the numerator does not have a non-analytic form such as $\sqrt{q_{k}^{*}-a^{*}}$, which would fail to cancel the singularity.

A special case occurs if $q_{k}^{*} \rightarrow 0$, for then there is a double pole in $a^{*}$. In addition, one might be concerned about the factor of $1 /\left(q_{k}^{*}\right)^{\ell^{\prime}+\ell}$. These features do not, however, lead to problems. We know from Eqs. D.7 and D.8 that $g_{\ell^{\prime}, m^{\prime}}^{*}(\vec{k}) \propto q_{k}^{* \ell}$, and similarly for $h$, so the $1 / q_{k}^{*}$ factors are cancelled. Furthermore, because of Eq. (D.7), the difference in the numerator of $(\mathrm{D} .5)$ is proportional to $a^{* 2}$, and thus fully cancels the double pole.

This completes the demonstration of the key identity. We close this section by presenting some further results for the kinematic functions $F$ and $F^{i \epsilon}$. First, we give the relation to the kinematic functions $c^{P}$ introduced in Ref. [130]. These replace the product $g \times h$ with a single function, which is then expanded in a single-set of spherical harmonics. Because of this, the relation involves Clebsch-Gordon coefficients. Specifically we find (see also Ref. [140]):1]

$$
F_{\ell^{\prime}, m^{\prime} ; \ell, m}^{i \epsilon}(\vec{k})=\frac{i q_{k}^{*}}{16 \pi E_{2, k}^{*}} \delta_{\ell^{\prime}, \ell} \delta_{m^{\prime}, m}+\sum_{\widetilde{\ell}, \widetilde{m}} \frac{\sqrt{4 \pi} c_{\widetilde{\ell}, \widetilde{m}}^{P}\left(q_{k}^{*}\right)}{4 E_{2, k}^{*}\left(q_{k}^{*}\right)^{\widetilde{\ell}}} \int d \Omega_{a^{*}} Y_{\widetilde{\ell}, \widetilde{m}}^{*}\left(\hat{a}^{*}\right) Y_{\ell^{\prime}, m^{\prime}}\left(\hat{a}^{*}\right) Y_{\ell, m}^{*}\left(\hat{a}^{*}\right) .
$$

Because Ref. [130] uses an exponential cut-off while we use $H(\vec{a}) H\left(\vec{b}_{k a}\right)$, this result holds only up to exponentially suppressed finite-volume corrections $2^{2}$

\footnotetext{
${ }^{1}$ One subtlety in the derivation of Eq. D.10 is that the powers of $a^{*} / q_{k}^{*}$ do not always match. This is because we use a double expansion in spherical harmonics while Ref. [130] use a single expansion. One can show, however, that the differences always lead to exponentially suppressed contributions.

${ }^{2}$ One might wonder why we use the $H$ functions to provide the cut-off, since, as far as sum-integral identity is concerned, we could use any reasonable cut-off. The reason we use $H$ is that some of the factors of $F$ arise from insertions of the quantity $G$ [Eq. (5.21)]. But $G$ contains, as part of its essential definition, two factors of $H$, one of which becomes $H(\vec{a})$ when we convert the $G$ to an $F$. Thus an $H$ cut-off is forced
} 
Finally, we derive a result needed in the main text, namely

$$
F_{\ell^{\prime}, m^{\prime} ; \ell, m}(\vec{k})=F_{\ell^{\prime}, m^{\prime} ; \ell, m}^{i \epsilon}(\vec{k})=0 \text { if } \ell^{\prime}+\ell=\text { odd }
$$

up to exponentially suppressed corrections. It is sufficient to show this result for one of $F$ and $F^{i \epsilon}$, since it holds trivially for their difference, $\rho$, which is diagonal in angular momentum. We demonstrate D.11 for $F^{i \epsilon}$.

The result follows by averaging the original expression (D.3) with that obtained by changing variables $\vec{a} \rightarrow \vec{P}-\vec{a}=\vec{b}_{k a}$. In this way, the numerator of $F^{i \epsilon}$ is replaced, up to an overall constant, with

$$
\left[Y_{\ell^{\prime}, m^{\prime}}\left(\hat{a}^{*}\right) Y_{\ell, m}^{*}\left(\hat{a}^{*}\right)\left(a^{*}\right)^{\ell^{\prime}+\ell}+Y_{\ell^{\prime}, m^{\prime}}\left(\hat{b}_{k a}^{*}\right) Y_{\ell, m}^{*}\left(\hat{b}_{k a}^{*}\right)\left(b_{k a}^{*}\right)^{\ell^{\prime}+\ell}\right] H(\vec{a}) H\left(\vec{b}_{k a}\right) .
$$

If all particles are on shell, then from Eq. 5.5 , we have that $\vec{a}^{*}=-\vec{b}_{k a}^{*}$, so the two terms exactly cancel when the parities of the spherical harmonics are opposite, i.e. if $\ell+\ell^{\prime}$ is odd 3 As we move away from the on-shell condition, the cancellation will be inexact. However, as we now demonstrate, the residue is proportional to $E_{2}-\omega_{a}-\omega_{k a}$, which is enough to cancel the pole in $F^{i \epsilon}$, so that the sum-integral difference of the residue is exponentially suppressed. We recall that the boost to the two-particle CM frame transforms four-vectors as

$$
\left(E_{2}-\omega_{a}, \vec{b}_{k a}\right) \longrightarrow\left(E_{2, k}^{*}-\omega_{a}^{*},-\vec{a}^{*}\right) \text { and }\left(\omega_{k a}, \vec{b}_{k a}\right) \longrightarrow\left(\omega_{b}^{*}, \vec{b}_{k a}^{*}\right)
$$

But since

$$
\left(\omega_{k a}, \vec{b}_{k a}\right)=\left(E_{2}-\omega_{a}, \vec{b}_{k a}\right)-\left(E_{2}-\omega_{a}-\omega_{k a}, \overrightarrow{0}\right)
$$

we see from the linearity of boosts that

$$
\vec{b}_{k a}^{*}=-\vec{a}^{*}+\mathcal{O}\left(E_{2}-\omega_{a}-\omega_{k a}\right)
$$

This completes the demonstration.

upon us for such $F$ 's, and we wish to use a uniform definition. It is then convenient to enforce $a \leftrightarrow b_{k a}$ symmetry by adding in $H\left(\vec{b}_{k a}\right)$.

${ }^{3}$ It is possible for $\vec{a}=\vec{P} / 2=\vec{b}_{k a}$, and if so the two terms in the sum are one and the same. The derivation remains valid, however, since then $\vec{a}^{*}=0$, implying that the only non-vanishing contributions are from $\ell=\ell^{\prime}=0$. 
We note that a similar argument leads to the conclusion that $c_{\ell, m}^{P}$ vanishes for odd $\ell$, as first noted in Ref. [129]. 


\section{Appendix E \\ SMOOTHNESS OF $\widetilde{P V}$ POLE PRESCRIPTION}

In this appendix we explain why the $\widetilde{\mathrm{PV}}$ pole prescription, defined in Eq. 5.59 , leads to results that are smooth functions of the spectator momentum. The general integral that appears has the form

$$
f(\vec{k})=\widetilde{\mathrm{PV}} \int_{\vec{a}} \frac{g(\vec{k}, \vec{a}) H(\vec{k})}{2 \omega_{a} 2 \omega_{k a}\left(E-\omega_{k}-\omega_{a}-\omega_{k a}\right)}
$$

The notation is the same as in Eq. (D.1), except that here we have combined the two functions $g$ and $h$ in the numerator of (D.1) into the single function $g$. The issue is whether $f(\vec{k})$ is a smooth function of $\vec{k}$. All quantities appearing in the integrand are smooth functions: $\omega_{k}$ and $\omega_{k a}$ manifestly, $H(\vec{k})$ by construction, and $g(\vec{k}, \vec{a})$ by assumption 11 We also assume that the behavior of $g$ at large $|\vec{a}|$ is such that the integral remains convergent however many derivatives of the integrand with respect to the components of $\vec{k}$ we take. Then the only source for a lack of smoothness is the pole in the integrand.

It is useful to change variables to $\vec{a}^{*}$, the momentum in the two-particle CM frame. This gives

$$
\begin{aligned}
f(\vec{k}) & =\frac{H(\vec{k})}{2 E_{2, k}^{*}} \widetilde{\mathrm{PV}} \int \frac{d^{3} a^{*}}{(2 \pi)^{3}} \frac{\widetilde{g}^{*}\left(\vec{k}, \vec{a}^{*}\right)}{\left(q_{k}^{* 2}-a^{* 2}\right)} \\
\widetilde{g}^{*}\left(\vec{k}, \vec{a}^{*}\right) & =g(\vec{k}, \vec{a}) \frac{\left(E-\omega_{k}-\omega_{a}+\omega_{k a}\right)\left(E_{2, k}^{*}+2 \omega_{a}^{*}\right)}{8 \omega_{k a} \omega_{a}^{*}} .
\end{aligned}
$$

\footnotetext{
${ }^{1}$ The initial application of the result of this appendix, in the discussion following Eq. 5.65 in the main text, has $g$ composed of the product $\sigma \sigma^{\dagger}$, which is smooth by construction. Subsequently, one or both of these factors are replaced by Bethe-Salpeter kernels, which are also smooth because singularities are far from threshold. The nearest singularity is the left-hand cut which occurs when $E_{2, k}^{*}=0$ (corresponding to $s=u=0, t=4 m^{2}$ in Mandlestam variables), but we are protected from this cut by the cut-off function $H(\vec{k})$. Finally, the factors are replaced by two particle K-matrices, or decorated end-cap functions. Here the necessary smoothness is established by the argument of this appendix. Thus we are using the result of this appendix iteratively.
} 
The expression multiplying $g$ on the right-hand side of (E.3) equals unity on shell. Expanding $\widetilde{g}^{*}$ in spherical harmonics,

$$
\widetilde{g}^{*}\left(\vec{k}, \vec{a}^{*}\right)=\widetilde{g}_{\ell, m}^{*}\left(\vec{k}, a^{*}\right) Y_{\ell, m}\left(\hat{a}^{*}\right) \sqrt{4 \pi}
$$

we observe that only the $\ell=0$ component contributes to the integral:

$$
f(\vec{k})=\frac{H(\vec{k})}{4 \pi^{2} E_{2, k}^{*}} \widetilde{\mathrm{PV}} \int_{0}^{\infty} d a^{*} \frac{a^{* 2} \widetilde{g}_{0,0}^{*}\left(\vec{k}, a^{* 2}\right)}{\left(q_{k}^{* 2}-a^{* 2}\right)}=\frac{H(\vec{k})}{8 \pi^{2} E_{2, k}^{*}} \widetilde{\mathrm{PV}} \int_{0}^{\infty} d\left(a^{* 2}\right) \frac{\sqrt{a^{* 2}} \widetilde{g}_{0,0}^{*}\left(\vec{k}, a^{* 2}\right)}{\left(q_{k}^{* 2}-a^{* 2}\right)}
$$

Here we have made explicit that $\widetilde{g}_{0,0}^{*}$ is a function of $a^{* 2}$, as follows from the result (D.7).

In this form, the $\widetilde{\mathrm{PV}}$ prescription of Eq. 5.59 becomes

$$
\begin{aligned}
& f(\vec{k})=\frac{H(\vec{k})}{8 \pi^{2} E_{2, k}^{*}} \int_{0}^{\infty} d\left(a^{* 2}\right)\left[\frac{\sqrt{a^{* 2}} \widetilde{g}_{0,0}^{*}\left(\vec{k}, a^{* 2}\right)}{\left(q_{k}^{* 2}-a^{* 2}+i \epsilon\right)}\right] \\
& \quad+\frac{i q_{k}^{*} H(\vec{k}) \widetilde{g}_{0,0}^{*}\left(\vec{k}, q_{k}^{* 2}\right)}{8 \pi E_{2, k}^{*}} \times \begin{cases}i q_{k}^{*} & \left(q_{k}^{* 2}>0\right), \\
-\left|q_{k}^{*}\right| & \left(q_{k}^{* 2}<0\right) .\end{cases}
\end{aligned}
$$

If $q_{k}^{* 2}>0$, so the non-spectator pair is above threshold, the $\widetilde{\mathrm{PV}}$ and $\mathrm{PV}$ prescriptions are the same, and Eq. (E.6) gives the standard relationship between PV and $i \epsilon$ prescriptions. In particular, the second term cancels the imaginary part of the $i \epsilon$-regulated integral. The new feature of the prescription occurs below threshold, i.e. for $q_{k}^{* 2}<0$. Here there is no pole to regulate, so the $i \epsilon$ prescription is superfluous, and the integral is real. Nevertheless, the prescription adds the second term, also real, which is needed to avoid a cusp in $f(\vec{k})$ at threshold. We stress that in the second term $\widetilde{g}^{*}$ is evaluated on shell, with $a^{* 2}=q_{k}^{* 2}$. If $q_{k}^{* 2}<0$, then $\widetilde{g}^{*}$ must be evaluated below threshold. As discussed in the previous appendix, the assumed smoothness of $g$ implies that $\widetilde{g}_{0,0}^{*}$ is a function of $q_{k}^{* 2}$, and thus can be straightforwardly evaluated for $q_{k}^{* 2}<0$.

To show that $f(\vec{k})$ is smooth, we now extract the essential features of Eq. E.6 and consider the integral

$$
f(z)=\int_{0}^{\infty} d w \frac{\sqrt{w} g(w, z)}{z-w} .
$$

Here $w$ and $z$ are playing the roles of $a^{* 2}$ and $q_{k}^{* 2}$, respectively, and $g$, a smooth function of its arguments, ensures convergence (and includes $H(\vec{k})$ ). The only difference from Eq. E.6. 
is that the dependence on all components of $\vec{k}$ in $H$ and $\widetilde{g}^{*}$ has been simplified to dependence on $q_{k}^{* 2}$ alone. This simplification is justified because it is the dependence of the pole term on $\vec{k}$ that can lead to a lack of smoothness, and this dependence is correctly incorporated in Eq. E.7).

We treat $z$ as complex, and assume that $g(w, z)$ can be analytically continued to complex arguments without encountering singularities. Then $f(z)$ is well-defined and analytic in the entire complex plane except along the positive real axis. As $z$ approaches the positive real axis from above or below one obtains the $\pm i \epsilon$-regulated integrals:

$$
f(x \pm i \epsilon)=\int_{0}^{\infty} d w \frac{\sqrt{w} g(w, x)}{x-w \pm i \epsilon} .
$$

These are both complex, with the same real parts but differing imaginary parts, $\pm \pi i \sqrt{x} g(x, x) . f(z)$ thus has a cut on the positive real axis.

The integral of interest, $f(\vec{k})$ of Eq. E.5), becomes, in our stripped-down version, and for positive $q_{k}^{* 2}$

$$
f_{\widetilde{\mathrm{PV}}}(x)=\frac{1}{2}[f(x+i \epsilon)+f(x-i \epsilon)] .
$$

Here $x$ is real and positive, and we have used the result that the PV prescription (which is the same as the $\widetilde{\mathrm{PV}}$ prescription for $x>0$ ) can be written as the average of the integral with the contour running above and below the pole (see Fig. E.1 1 ). Our aim is to extend $f_{\widetilde{\mathrm{PV}}}$ to a function of $z$, and study its analyticity properties.

If $z$ is moved off the positive real axis then, to avoid non-analytic dependence, the integration contours must be deformed as shown in Fig E.1p and Fig E.1. Thus $f_{\widetilde{\mathrm{PV}}}(z)$ differs from $f(z)$ (the integral along the real axis) by pole terms:

$$
f_{\widetilde{\mathrm{PV}}}(z)=f(z)-\operatorname{sign}(\operatorname{Im} z) i \pi \sqrt{z} g(z, z)=f(z)+\pi \sqrt{-z} g(z, z)
$$

The sign of $\operatorname{Im}(z)$ enters because the direction of the contour around the pole depends on this sign. As shown in the second expression, however, the two possibilites can be combined into a single expression using the properties of the square root (assuming that the branch cut for $\sqrt{-z}$ is placed along the positive real axis). We now observe that there is no discontinuity for negative real $z$, since both $f(z)$ and $\sqrt{-z}$ are analytic there. Furthermore, by construction the discontinuities of $f(z)$ and the pole term cancel exactly along the positive real axis [as 
they must to yield a real $\left.f_{\widetilde{\mathrm{PV}}}(x)\right]$. Thus we find the key result: $f_{\widetilde{\mathrm{PV}}}(z)$ is analytic throughout the complex plane, i.e. is entire. Since an entire function is infinitely differentiable, it follows that $f_{\widetilde{\mathrm{PV}}}(x)$ is smooth along the entire real axis.

We now apply this result to our integral of interest, Eq. E.5. The rule is to write the difference between the results of the $\widetilde{\mathrm{PV}}$ and $i \epsilon$ prescriptions, which is standard above threshold, as an analytic function of $q_{k}^{* 2}$, and then continue to $q_{k}^{* 2}<0$. Noting that

$$
i q_{k}^{*}=-\sqrt{-q_{k}^{* 2}} \text { for } q_{k}^{* 2}=x+i \epsilon
$$

we obtain the result quoted in E.5 for the below threshold case

$$
-\sqrt{-q_{k}^{* 2}} \longrightarrow-\left|q_{k}^{*}\right| \text { for } q_{k}^{* 2}=-x
$$

Thus our $\widetilde{\mathrm{PV}}$ prescription indeed yields a smooth function of $\vec{k}$.

We have checked this result on an extensive set of examples, e.g. for $g(w, z)=$ $w^{n} \exp (-w)$ with $n \geq 0$ an integer, where the integrals can be done analytically, and for $g(w, z)=\exp \left(-w^{2}\right)$, where numerical integration is required. As an illustration, we show the results for $g(w, z)=\exp (z-w)$ in Fig. E.2.

Finally, we stress that the factor of $\sqrt{w}$ in the integrand of $f(z)$ is crucial for the smoothness of the $\widetilde{\mathrm{PV}}$ prescription. This factor is present in the original integral, Eq. E.5, because of three-dimensional phase space. Without this factor, the above- and below-axis pole terms would not be equal along the negative real axis. For example, if $\sqrt{w}$ is replaced by an analytic function, say $x$, then one can easily show, using the arguments above, that $f_{\widetilde{\mathrm{PV}}}(z)$ result has a complex discontinuity along the negative real axis. 


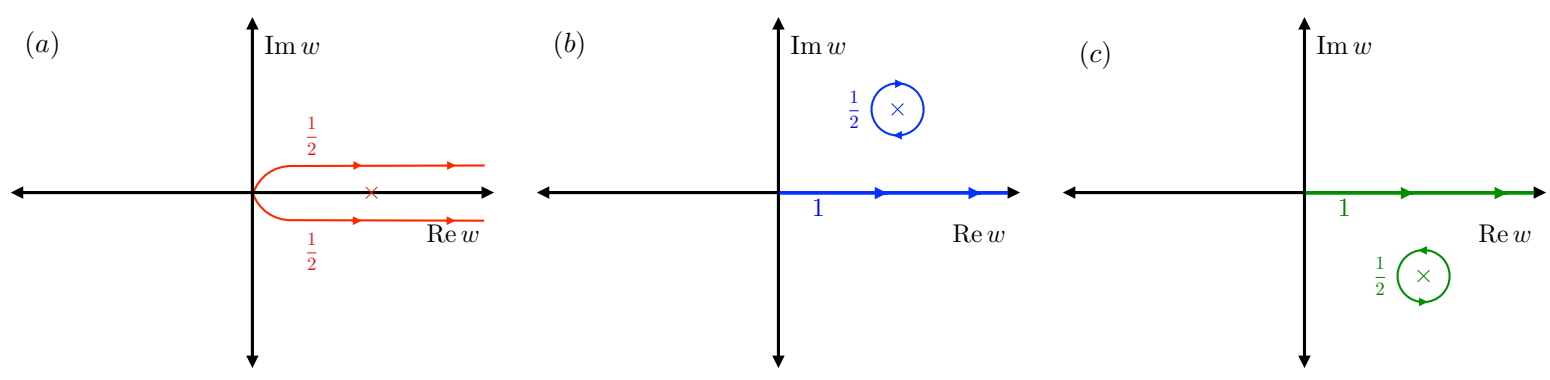

Figure E.1: Contours in $w$ complex plane contributing to $f_{\widetilde{\mathrm{PV}}}(z)$. (a) $z$ real and positive; (b) $z$ above the positive real axis; (c) $z$ below the positive real axis. In each case the cross indicates the location of the $z=w$ pole, and the numbers indicate the weights associated with each contour.

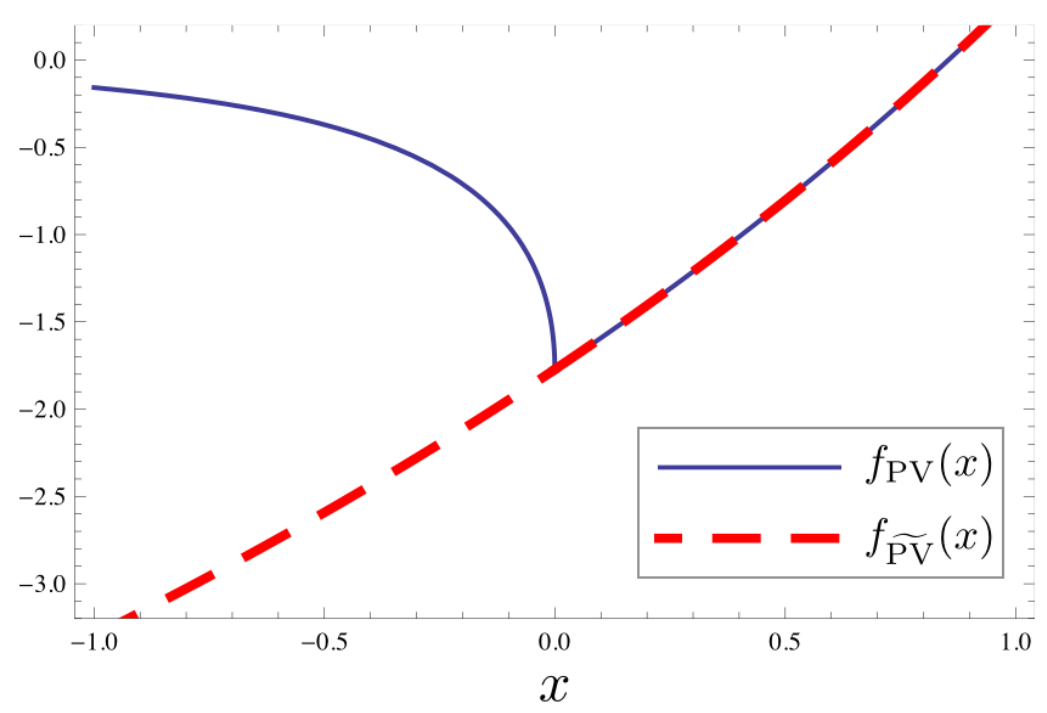

Figure E.2: $f_{\widetilde{\mathrm{PV}}}(x)$ for $g(w, z)=\exp (z-w)$ compared to the result of using the PV prescription, $f_{\mathrm{PV}}(x)$. The former is smooth, while the latter has a cusp at $x=0$. For $x<0$, the difference between the two functions is the pole term, the second term in Eq. (E.10). 
Appendix F

\section{DETAILED STUDY OF THE ISOTROPIC APPROXIMATION}

In this appendix we study the approach to the isotropic limit described in Sec. 5.2 in the context of a specific choice of parameters. Our aims are to show how "lost" states are recovered, and also to gain more intuition for the workings of the quantization condition and, in particular, the impact of the violation of particle-interchange symmetry by our coordinates and truncation. Although our considerations are specific to the chosen parameters, much of the discussion holds for a general choice of parameters.

We work in the static frame, $\vec{P}=0$. To simplify numerical values we choose a volume such that the particle mass satisfies $m=2 \pi / L$. While this is artificial, we stress that none of the general conclusions depend on this choice. The single-particle momenta are $(L / 2 \pi) \vec{k}=\overrightarrow{0},(1,0,0),(1,1,0),(1,1,1)$, etc., together with permutations. We refer to these, respectively, as the $n=0,1,2,3$ shells, etc. Given our choice of $m$, the corresponding single-particle energies are $\omega_{n}=\sqrt{n+1} \times m$.

We are interested in values of $E$ for which there are more than one free three-particle energy levels, so that we can see what happens when one of these is replaced by the solution to Eq. (5.38). The minimal case is to have two free levels. These levels occur at $E=3 \mathrm{~m}$ (all particles at rest), $E=(1+2 \sqrt{2}) m \approx 3.83 m$ (one particle at rest and two with opposite momenta from the $n=1$ shell), $E=4.46 m$, etc. Thus the range of $E$ should extend above $3.83 \mathrm{~m}$. We also want to minimize $N$, the size of the matrices appearing in the quantization

condition. The critical energies above which the cut-off function $H(\vec{k})$ is non-vanishing are, for the $n=1-4$ shells, $(1+\sqrt{2}) m,(\sqrt{2}+\sqrt{3}) m \approx 3.15 m,(2+\sqrt{3}) \approx 3.73 m$ and $(2+\sqrt{5}) m \approx 4.24 m$, respectively. Thus we are forced to include the $n=3$ shell (in order to attain $E>3.83 m$ ) but if we restrict $E<4.24 m$ we do not need the $n=4$ shell.

Thus we end up with the energy range of interest being $3 m<E<4.24 m$ and $N=$ 
$1+6+12+8=27$. Our aim is to find all solutions to the quantization condition,

$$
\operatorname{det}\left(1+F_{3}^{s} \mathcal{K}_{\mathrm{df}, 3}^{s}\right)=0
$$

within this range. Here $\mathcal{K}_{\mathrm{df}, 3}^{s}$ is defined in Eq. (5.14), while $F_{3}^{s}$ is simply $F_{3}$ [Eq. (5.19)] after truncation to $\ell_{\max }=0$. (An explicit expression is given below.) As already noted, the free states lie at $E=3 m$ and $3.83 m$. There are four such states: one at $3 m$ and three at $3.83 \mathrm{~m}$. One might have expected six states at $E=3.83 \mathrm{~m}$, since two of the particles have momenta in the $n=1$ shell, but only three are distinct for indentical particles. It is useful to classify the states according to their transformation properties under the octahedral symmetry group. The $E=3 m$ state lies in the trivial $A_{1}$ irrep (irreducible representation), while the three $E=3.83 m$ states decompose as $A_{1}+E$, where $E$ is the doublet. Here we are using standard notation for irreps of the cubic group, see, e.g., Ref. [251]. Interactions can lead to mixing between the two $A_{1}$ states, but not with the doublet. What we thus expect is that one of the two $A_{1}$ states is replaced by the solution to the isotropic quantization condition, Eq. (5.38), while the other remains at its free energy, and that the doublet also remain at their free energies.

As a first step in the analysis, it is useful to rewrite $F_{3}^{s}$ as ${ }^{1}$

$$
F_{3}^{s}=\frac{1}{L^{3}}\left\{\frac{1}{3} \frac{F^{s}}{2 \omega}-\frac{F^{s}}{2 \omega} \frac{1}{H} \frac{F^{s}}{2 \omega}\right\}, \quad H=\frac{1}{2 \omega \mathcal{K}_{2}^{s}}+\frac{F^{s}}{2 \omega}+\frac{1}{2 \omega} G^{s},
$$

where $\mathcal{K}_{2}^{s}, G^{s}$ and $F^{s}$ are defined, respectively, in Eqs. (5.34), 5.36) and (5.37). Recall that all these quantities, as well as $F_{3}^{s}$, are matrices in spectator-momentum space alone, with size $N \times N$. The form (F.2) follows by straightforward algebraic manipulations from the definition of Eq. (5.19). One advantage of the new form is that it manifests the symmetry of $F_{3}^{s}$, since $\widetilde{G}^{s}=\frac{1}{2 \omega} G^{s}$ is symmetric, and all other matrices are diagonal. Another is that it shows how $F^{s}$ appears on both "ends" of $F_{3}^{s}$.

The matrices entering the quantization condition have transformation properties under the symmetries of the finite box that greatly simplify their forms. Beginning with $\widetilde{F}_{s} \equiv$ $F_{s} /(2 \omega)$, it is clear from its definition, Eq. (5.37), that it is invariant under cubic rotations and parity. Thus its entries, which are all diagonal, depend only on the class $n$ of the

\footnotetext{
${ }^{1}$ We stress that the matrix $H$ used here has nothing to do with the smooth cutoff function $H(\vec{k})$.
} 
spectator momentum $\vec{k}$. For example, all 6 entries for the $n=1$ class are equal. Thus there are only 4 independent entries in $\widetilde{F}^{s}$. The same holds for the other diagonal matrix, $\mathcal{K}_{2}^{s}$.

The situation with $\widetilde{G}^{s}$ is more complicated-all entries are non-vanishing. To simplify $\widetilde{G}^{s}$ we must decompose the $N$ spectator-momentum indices into irreps of the finite-box symmetry group, namely the direct product of the cubic group and parity. The decomposition is

$$
\begin{aligned}
& n=0 \longrightarrow A_{1}^{+}, \\
& n=1 \longrightarrow A_{1}^{+}+E^{+}+T_{1}^{-}, \\
& n=2 \longrightarrow A_{1}^{+}+E^{+}+T_{1}^{-}+T_{2}^{+}+T_{2}^{-}, \\
& n=3 \longrightarrow A_{1}^{+}+T_{1}^{-}+T_{2}^{+}+A_{2}^{-},
\end{aligned}
$$

where the superscript is parity. $A_{2}$ is a non-trivial singlet, while $T_{1}$ and $T_{2}$ are threedimensional irreps. Off-diagonal elements of $\widetilde{G}^{s}$ connecting different irreps, or different elements of the same irrep, vanish. Thus $\widetilde{G}_{s}$ is block-diagonal, with a four-dimensional $A_{1}^{+}$ block, a $2 \times 2$-dimensional $E^{+}$block, etc.

The same block structure holds for $\mathcal{K}_{\mathrm{df}, 3}^{s}$, but here additional simplification occurs because of the isotropic approximation, Eq. 5.33. In this approximation, all entries of $\mathcal{K}_{\mathrm{df}, 3}^{s}$ are equal. Since the $A_{1}^{+}$irreps are obtained by averaging over their respective momentum shells, while all other irreps involve differences, only the $A_{1}^{+}$block of $\mathcal{K}_{\mathrm{df}, 3}^{s}$ is non-vanishing. Furthermore, since $\mathcal{K}_{\mathrm{df}, 3}^{s}$ has only a single non-zero eigenvalue, whose eigenvector we call $\left|1_{K}\right\rangle$, all entries of the $A_{1}^{+}$block are related. Here we will slightly relax the approximation, so that all entries which are allowed by symmetries have magnitudes of order $\epsilon \ll 1$. Thus we can write

$$
\mathcal{K}_{\mathrm{df}, 3}^{s}=\left|1_{K}\right\rangle N \mathcal{K}_{\mathrm{df}, 3}^{\mathrm{iso}}(E)\left\langle 1_{K}\right|+[\mathcal{O}(\epsilon)]
$$

where the second term indicates an $N \times N$ matrix with form consistent with the symmetries whose non-zero entries are of $\mathcal{O}(\epsilon)$ (though unrelated). If we choose the indices so that the $A_{1}^{+}$block is placed first, ordered according to the class $n$, the dominant eigenvector is easily found to be

$$
\left\langle 1_{K}\right|=\frac{1}{\sqrt{N}}(1, \sqrt{6}, \sqrt{12}, \sqrt{8}, 0, \ldots) .
$$


We now return to the quantization condition (F.1). Since all matrices which enter are diagonal or block-diagonal, the condition can be studied block by block. We begin with the $A_{1}^{+}$block, which is the most interesting as it contains the dominant eigenvector of $\mathcal{K}_{\mathrm{df}, 3}^{s}$. Since $\widetilde{F}^{s}$ and $\mathcal{K}_{2}^{s}$ were diagonal in the original basis, with no cross terms between different classes $n$, they remain diagonal in the irrep basis. In general, there are no relations between the four diagonal elements of the $A_{1}^{+}$blocks. As we approach the free-spectrum energies, however, $\widetilde{F}^{s}$ does gain further structure. This is because it contains poles at these energies [from the sum contained in $F^{s}$, Eq. (5.37)]. Specifically, one finds that

$$
\widetilde{F}_{s}=\operatorname{diag}\left(\frac{1}{2} B_{0}+3 B_{1}, B_{1}, 0,0\right)+\operatorname{diag}(\mathcal{O}(1))
$$

where the pole terms are

$$
\begin{aligned}
& B_{0}=\frac{1}{L^{3}} \frac{1}{(2 m)^{3}} \frac{1}{E-3 m}, \\
& B_{1}=\frac{1}{L^{3}} \frac{1}{2 m\left(2 \omega_{1}\right)^{2}} \frac{1}{E-m-2 \omega_{1}} .
\end{aligned}
$$

The factor of 3 multiplying $B_{1}$ in the first entry on the right-hand side of Eq. (F.9) arises from the fact that six terms in the sum over $\vec{a}$ in $F^{s}$ contribute $B_{1} / 2$. The second term in $\widetilde{F}^{s}$ is the non-pole part, arising from the rest of the sum over $\vec{a}$ and from the integral.

Pole terms also appear in $\widetilde{G}^{s}$. Using Eq. 5.21 we find the $A_{1}^{+}$block to be

$$
\widetilde{G}^{s}=\left(\begin{array}{cccc}
B_{0} & \sqrt{6} B_{1} & \mathcal{O}(1) & \mathcal{O}(1) \\
\sqrt{6} B_{1} & B_{1} & \mathcal{O}(1) & \mathcal{O}(1) \\
\mathcal{O}(1) & \mathcal{O}(1) & \mathcal{O}(1) & \mathcal{O}(1) \\
\mathcal{O}(1) & \mathcal{O}(1) & \mathcal{O}(1) & \mathcal{O}(1)
\end{array}\right)
$$

Apart from the fact that the matrix is symmetric, there is no relation between the $\mathcal{O}(1)$ 
terms. The combination whose inverse appears in $F_{3}^{s}[\mathrm{Eq} .(\mathrm{F} .2)]$ is thus

$$
H \equiv \frac{1}{2 \omega \mathcal{K}_{2}^{s}}+\widetilde{F}^{s}+\widetilde{G}^{s}=\left(\begin{array}{cccc}
\frac{3}{2} B_{0}+3 B_{1} & \sqrt{6} B_{1} & 0 & 0 \\
\sqrt{6} B_{1} & 2 B_{1} & 0 & 0 \\
0 & 0 & 0 & 0 \\
0 & 0 & 0 & 0
\end{array}\right)+[\mathcal{O}(1)]
$$

where the $\mathcal{O}(1)$ symmetric matrix now contains entries in all positions.

Our task is to combine these forms and insert them in the quantization condition. Since $B_{0}$ and $B_{1}$ become large for different regions of $E$, we treat these cases one at at time. The simpler is when $E \approx 3 m$, such that $\left|B_{0}\right| \gg 1$, while $B_{1} \sim \mathcal{O}(1)$. Using Raliegh-Schrödinger perturbation theory, one finds that the inverse of $H$ becomes

$$
H^{-1}=\left(\begin{array}{cc}
\frac{2}{3 B_{0}}+\mathcal{O}\left(1 / B_{0}^{2}\right) & \mathcal{O}\left(1 / B_{0}\right) \\
\mathcal{O}\left(1 / B_{0}\right) & \mathcal{O}(1)
\end{array}\right)
$$

Here we are using a block notation in which the first block has dimension one (the $n=0 A_{1}^{+}$ subspace) while the second block has dimension three (containing the $n=1-3 A_{1}^{+}$states). This result exemplifies two general features of $H^{-1}$ when a one-dimensional subspace of $H$ becomes large. First, the projection of $H^{-1}$ onto this subspace is, up to small corrections, simply the inverse of the projection of $H$ onto the subspace. Thus it is proportional to $1 / B_{0}$. Second, the off-diagonal elements of the inverse (those connecting the 1-d subspace to the remainder of the space) are of $\mathcal{O}\left(1 / B_{0}\right)$. We use these results again below. 
Combining the results above, we find that, when $\left|B_{0}\right| \gg 1$,

$$
\begin{aligned}
& L^{3} F_{3}^{s}=\frac{\widetilde{F}^{s}}{3}-\widetilde{F}_{s} H^{-1} \widetilde{F}_{s} \\
&=\left(\begin{array}{cc}
\frac{B_{0}}{6}+\mathcal{O}(1) & 0 \\
0 & \mathcal{O}(1)
\end{array}\right) \\
&-\left(\begin{array}{cc}
\frac{B_{0}}{2}+\mathcal{O}(1) & 0 \\
0 & \mathcal{O}(1)
\end{array}\right)\left(\begin{array}{cc}
\frac{2}{3 B_{0}}+\mathcal{O}\left(1 / B_{0}^{2}\right) & \mathcal{O}\left(1 / B_{0}\right) \\
\mathcal{O}\left(1 / B_{0}\right) & \mathcal{O}(1)
\end{array}\right)\left(\begin{array}{cc}
\frac{B_{0}}{2}+\mathcal{O}(1) & 0 \\
0 & \mathcal{O}(1)
\end{array}\right) \\
&=[\mathcal{O}(1)],
\end{aligned}
$$

using the same $1+3$ block notation as in Eq. F.14). The key result is that all terms proportional to positive powers of $B_{0}$ cancel. When we combine (F.18) with the result (F.7) for $\mathcal{K}_{\mathrm{df}, 3}^{s}$ and evaluate the determinant, the quantization condition becomes

$$
\operatorname{det}\left(1+F_{3}^{s} \mathcal{K}_{\mathrm{df}, 3}^{s}\right)=1+N \mathcal{K}_{\mathrm{df}, 3}^{i s o}(E)\left\langle 1_{K}\left|F_{3}^{s}\right| 1_{K}\right\rangle+\mathcal{O}(\epsilon)=0
$$

We see that there is only a single solution in the $A_{1}^{+}$channel, that given essentially by the "isotropic solution" of Eq. (5.38), aside from small corrections from the $O(\epsilon)$ terms. There is no possibility of a solution that is $\mathcal{O}(\epsilon)$ from $E=3 \mathrm{~m}$, because there are no terms of the form $\epsilon B_{0}$ or $\epsilon B_{0}^{2}$, which could have led to $\mathcal{O}(1)$ contributions to the quantization condition. Such terms are required to cancel the 1 in Eq. (F.19), in order to get solutions that are infinitesimally displaced from the the free solution.

The analysis near $E=m+2 \omega_{1}$, when $\left|B_{1}\right| \gg 1$, is more involved, for in this case the free poles do not cancel. Using Eq. F.13, $H$ is now given by

$$
H=\left|B_{1}\right\rangle 5 B_{1}\left\langle B_{1}\right|+\mathcal{O}(1),
$$

where the one-dimensional subspace in which $H$ is large is spanned by

$$
\left\langle B_{1}\right|=\frac{1}{\sqrt{5}}(\sqrt{3}, \sqrt{2}, 0,0) .
$$


Using the results quoted above, we have

$H^{-1}=\left|B_{1}\right\rangle\left[\frac{1}{5 B_{1}}+\mathcal{O}\left(1 / B_{1}^{2}\right)\right]\left\langle B_{1}\left|+\sum_{j=2}^{4} \mathcal{O}\left(1 / B_{1}\right)\left(\left|B_{j}\right\rangle\left\langle B_{1}|+| B_{1}\right\rangle\left\langle B_{j}\right|\right)+\sum_{j=2}^{4} \mathcal{O}(1)\right| B_{j}\right\rangle\left\langle B_{j}\right|$,

where the $\left|B_{j}\right\rangle, j=2-4$, are any choice of basis vectors orthogonal to $\left|B_{1}\right\rangle$.

To display $F_{3}^{s}$ it is better to switch from the ordering of $A_{1}^{+}$elements according to their momentum class to the "K-basis". In this new basis, the vectors are $\left\langle 1_{K}\right|$ together with

$$
\begin{aligned}
& \left\langle 2_{K}\right|=\frac{1}{\sqrt{7}}(-\sqrt{6}, 1,0,0), \\
& \left\langle 3_{K}\right|=\frac{1}{\sqrt{N}}(-\sqrt{20 / 7},-\sqrt{120 / 7}, \sqrt{84 / 20}, \sqrt{56 / 20}), \\
& \left\langle 4_{K}\right|=\frac{1}{\sqrt{20}}(0,0,-\sqrt{8}, \sqrt{12}) .
\end{aligned}
$$

This basis makes maximal use of the form of $\mathcal{K}_{\mathrm{df}, 3}^{s}[\mathrm{Eq} .[$ F.7 $]$ as well as the fact that the dominant terms in $\widetilde{F}^{s}$ lie in the first two entries on the diagonal. In addition, we note that $\left\langle 1_{K}\right|$ and $\left\langle 3_{K}\right|$, when contracted with $\widetilde{F}^{s}$, have large components only in the subspace in which $H^{-1}$ is small:

$$
\begin{aligned}
\left\langle 1_{K}\right| \widetilde{F}^{s} & =\sqrt{\frac{15}{7}} B_{1}\left\langle B_{1}\right|+\mathcal{O}(1), \\
\left\langle 3_{K}\right| \widetilde{F}^{s} & =\sqrt{\frac{15}{7}} B_{1}\left\langle B_{1}\right|+\mathcal{O}(1) .
\end{aligned}
$$

Also important is that no large terms appear when we contract $\left\langle 4_{K}\right|$ with $\widetilde{F}^{s}$ :

$$
\left\langle 4_{K}\right| \widetilde{F}^{s}=\mathcal{O}(1)
$$

The net effect of these results is that the largest contributions occur when $F_{3}^{s}$ is contracted with $\left\langle 2_{K}\right|$. Specifically, we find

$$
F_{3}^{s}=\left(\begin{array}{cccc}
\mathcal{O}(1) & \mathcal{O}\left(B_{1}\right) & \mathcal{O}(1) & \mathcal{O}(1) \\
\mathcal{O}\left(B_{1}\right) & \mathcal{O}\left(B_{1}^{2}\right) & \mathcal{O}\left(B_{1}\right) & \mathcal{O}\left(B_{1}\right) \\
\mathcal{O}(1) & \mathcal{O}\left(B_{1}\right) & \mathcal{O}(1) & \mathcal{O}(1) \\
\mathcal{O}(1) & \mathcal{O}\left(B_{1}\right) & \mathcal{O}(1) & \mathcal{O}(1)
\end{array}\right)_{K}
$$


where the subscript on the matrix indicates that this result is in the K-basis. We see that $F_{3}^{s}$ does contain single and double $B_{1}$ pole terms (unlike for $B_{0}$, where they cancel). There is one cancellation, however, which leads to the absence of pole terms in the $\left|1_{K}\right\rangle\left\langle 1_{K}\right|$ element. This is important, since we know from Eq. (F.7) that the only large entry in $\mathcal{K}_{\mathrm{df}, 3}^{s}$ is in exactly this element:

$$
\mathcal{K}_{\mathrm{df}, 3}^{s}=\left(\begin{array}{cccc}
N \mathcal{K}_{\mathrm{df}, 3}^{\text {iso }}(E) & \mathcal{O}(\epsilon) & \mathcal{O}(\epsilon) & \mathcal{O}(\epsilon) \\
\mathcal{O}(\epsilon) & \mathcal{O}(\epsilon) & \mathcal{O}(\epsilon) & \mathcal{O}(\epsilon) \\
\mathcal{O}(\epsilon) & \mathcal{O}(\epsilon) & \mathcal{O}(\epsilon) & \mathcal{O}(\epsilon) \\
\mathcal{O}(\epsilon) & \mathcal{O}(\epsilon) & \mathcal{O}(\epsilon) & \mathcal{O}(\epsilon)
\end{array}\right)_{K}
$$

The final step of the $B_{1}$ analysis is to insert these results into the quantization condition (F.1) and evaluate the determinant. We are looking for solutions which occur when $B_{1}$ is large, so that they are almost at the free-particle energy. By explicit evaluation, we find that the dominant contributions to the determinant involving $B_{1}$ are of $\mathcal{O}\left(\epsilon B_{1}^{2}\right)$. Thus the appropropriate scaling of $B_{1}$ relative to $\epsilon$ is such that $\epsilon B_{1}^{2}=\mathcal{O}(1)$. Using this scaling, it turns out that only the upper-left $2 \times 2$ blocks in the K-basis are relevant for solutions to the quantization condition. Other blocks lead to contributions proportional to $\epsilon B_{1}$, which remains small. Thus, for the purpose of finding solutions to the quantization condition we can make the replacements

$$
F_{3}^{s} \longrightarrow\left(\begin{array}{cccc}
\mathcal{O}(1) & \mathcal{O}\left(B_{1}\right) & 0 & 0 \\
\mathcal{O}\left(B_{1}\right) & \mathcal{O}\left(B_{1}^{2}\right) & 0 & 0 \\
0 & 0 & 0 & 0 \\
0 & 0 & 0 & 0
\end{array}\right) \text { and } \mathcal{K}_{\mathrm{df}, 3}^{s} \longrightarrow\left(\begin{array}{cccc}
N \mathcal{K}_{\mathrm{df}, 3}^{\text {iso }}(E) & \mathcal{O}(\epsilon) & 0 & 0 \\
\mathcal{O}(\epsilon) & \mathcal{O}(\epsilon) & 0 & 0 \\
0 & 0 & 0 & 0 \\
0 & 0 & 0 & 0
\end{array}\right)
$$

This shows that, in the $A_{1}^{+}$block, the quantization condition involves only two states, and 
not four:

$$
\operatorname{det}\left(\begin{array}{cc}
1+N \mathcal{K}_{\mathrm{df}, 3}^{\text {iso }}(E)\left\langle 1_{K}\left|F^{s}\right| 1_{K}\right\rangle+\mathcal{O}\left(\epsilon B_{1}\right) & \mathcal{O}\left(\epsilon B_{1}\right) \\
\mathcal{O}\left(B_{1}\right) & 1+\mathcal{O}\left(\epsilon B_{1}^{2}\right)
\end{array}\right)=0
$$

When $E$ is far from $m+2 \omega_{1}$, so that $B_{1}=\mathcal{O}(1)$, the $B_{1}$ terms are small, and one finds only the isotropic solution, Eq. (5.38). But now, when $\epsilon B_{1}^{2}=\mathcal{O}(1)$, there is the possibility of a second solution. To demonstrate the existence and to find position of this solution, however, appears to require knowledge of the subdominant parts of $F^{s}, \mathcal{K}_{2}^{s}$ and $\mathcal{K}_{\mathrm{df}, 3}^{s}$. Nevertheless, what is clear is that any solution will lie very close to the free-particle energy, since it will require $\left|B_{1}\right| \gg 1$.

We now turn to blocks of the matrices in other irreps. These can only lead to solutions close to free-particle energies since $\mathcal{K}_{\mathrm{df}, 3}^{s}$ is of $\mathcal{O}(\epsilon)$ throughout these blocks. Such solutions require factors of $B_{1}$ to counterbalance those of $\epsilon$. $B_{1}$ appears in $\widetilde{F}^{s}$ in all diagonal elements with spectator momentum of class $n=1$, and thus [see Eq. (F.4)] appears in both $E^{+}$and $T_{1}^{-}$blocks. The same can be seen to hold for $\widetilde{G}^{s}$.

We consider first the $E^{+}$block. This has dimension four, since there are $E^{+}$irreps in both $n=1$ and $n=2$ classes, while the $E^{+}$irrep itself is a doublet. The structure within each $E^{+}$irrep is, however, always proportional to the identity matrix. Thus we display the blocks as $2 \times 2$ matrices, each element of which is implicitly proportional to the identity matrix. The matrices have the form

$$
\widetilde{F}^{s}=\left(\begin{array}{cc}
B_{1}+\mathcal{O}(1) & 0 \\
0 & \mathcal{O}(1)
\end{array}\right), H=\left(\begin{array}{cc}
2 B_{1}+\mathcal{O}(1) & \mathcal{O}(1) \\
\mathcal{O}(1) & \mathcal{O}(1)
\end{array}\right), \mathcal{K}_{\mathrm{df}, 3}^{s}=\left(\begin{array}{cc}
\mathcal{O}(\epsilon) & \mathcal{O}(\epsilon) \\
\mathcal{O}(\epsilon) & \mathcal{O}(\epsilon)
\end{array}\right)
$$

from which it follows that

$$
F_{3}^{s}=\left(\begin{array}{cc}
-\frac{B_{1}}{6}+\mathcal{O}(1) & \mathcal{O}(1) \\
\mathcal{O}(1) & \mathcal{O}(1)
\end{array}\right), 1+F_{3}^{s} \mathcal{K}_{\mathrm{df}, 3}^{s}=\left(\begin{array}{cc}
1+\mathcal{O}\left(\epsilon B_{1}\right) & \mathcal{O}\left(\epsilon B_{1}\right) \\
\mathcal{O}(\epsilon) & 1+\mathcal{O}(\epsilon)
\end{array}\right)
$$

Thus the determinant is $1+\mathcal{O}\left(\epsilon B_{1}\right)$, and can vanish if $\epsilon B_{1}=\mathcal{O}(1)$. In this case we know that such a solution will exist, irrespective of the overall sign of the $\epsilon B_{1}$ term, since $B_{1}$ can 
take either sign, depending on whether $E$ is above or below $m+2 \omega_{1}$. Thus we conclude that the $E^{+}$irrep yields a solution with $E \approx m+2 \omega_{1}$. Recalling the implicit $2 \times 2$ identity matrix in each entry, this will be a degenerate doublet.

At this stage we have all uncovered all the solutions we want-four in all (assuming that the $A_{1}^{+}$quantization condition does have an almost-free solution). But there remains the $T_{1}^{-}$block in which both $\widetilde{F}^{s}$ and $\widetilde{G}^{s}$ have entries of $B_{1}$. If these end up multiplying factors of $\epsilon$, as in the $E^{+}$block, then there is potential for unwanted solutions to the quantization condition, corresponding to states which violate particle-interchange symmetry. The way in which the formalism avoids this is through the particle-interchange symmetry that has been carefully maintained in $\mathcal{K}_{\mathrm{df}, 3}$. The issue is subtle, however, because our coordinates, and, in particular, the truncation we are using, is not particle-interchange symmetric.

The $T_{1}^{-}$block has contributions from classes $n=1,2$ and 3 , each of which is threedimensional, so the overall block dimension is nine. Entries within each $3 \times 3$ sub-block are, however, proportional to the identity matrix, so we leave this implicit and display only the $3 \times 3$ matrix indexed by momentum class. We find

$$
\widetilde{F}^{s}=\left(\begin{array}{ccc}
B_{1}+\mathcal{O}(1) & 0 & 0 \\
0 & \mathcal{O}(1) & 0 \\
0 & 0 & \mathcal{O}(1)
\end{array}\right), \quad \widetilde{G}^{s}=\left(\begin{array}{ccc}
-B_{1}+\mathcal{O}(1) & \mathcal{O}(1) & \mathcal{O}(1) \\
\mathcal{O}(1) & \mathcal{O}(1) & \mathcal{O}(1) \\
\mathcal{O}(1) & \mathcal{O}(1) & \mathcal{O}(1)
\end{array}\right)
$$

where the minus sign on $B_{1}$ in $\widetilde{G}^{s}$ arises from the negative parity of the $T_{1}^{-}$irrep and the fact that the non-zero elements of $\widetilde{G}^{s}$ in the original basis are those connecting an $n=1$ momentum to its parity conjugate. It follows from (F.35) that $B_{1}$ cancels from $H$, so that $H^{-1}$ is a general symmetric $\mathcal{O}(1)$ matrix with no small elements. We then find

$$
F_{3}^{s}=\left(\begin{array}{ccc}
\mathcal{O}\left(B_{1}^{2}\right) & \mathcal{O}\left(B_{1}\right) & \mathcal{O}\left(B_{1}\right) \\
\mathcal{O}\left(B_{1}\right) & \mathcal{O}(1) & \mathcal{O}(1) \\
\mathcal{O}\left(B_{1}\right) & \mathcal{O}(1) & \mathcal{O}(1)
\end{array}\right)
$$


so that, as in the $E^{+}$block, $F_{3}^{s}$ contains single and double poles.

If $\mathcal{K}_{\mathrm{df}, 3}^{s}$ was simply a symmetric matrix containing terms of $\mathcal{O}(\epsilon)$, then an analysis similar to that for the $E^{+}$block would imply the presence of almost-free solutions to the quantization condition in the $T_{1}^{-}$block. These would be unexpected, and indicate that our formalism was violating particle-interchange symmetry in a fundamental way. We are saved from this conclusion by the presence of additional structure in $\mathcal{K}_{\mathrm{df}, 3}^{s}$, following, not surprisingly, from particle-interchange symmetry. We recall that, before truncation, $\mathcal{K}_{\mathrm{df}, 3}$ is, by construction, exactly symmetric under particle interchange. We argue below that a consequence of this symmetry is that, if $E=m+2 \omega_{1}$ (so that the free three-particle state is exactly on shell), then

$$
\left[\mathcal{K}_{\mathrm{df}, 3}^{s}\right]_{(1,0,0) ; k^{\prime}}=\left[\mathcal{K}_{\mathrm{df}, 3}^{s}\right]_{(-1,0,0) ; k^{\prime}},
$$

(and similarly for the permutations of the left-hand momentum index). Here the right-hand index indicates an arbitrary momentum. The key feature of this result is that only the first index is parity-inverted - the second is unchanged. This implies that the projection on the left-hand index onto the irrep $T_{1}^{-}$, which involves taking the difference between the two sides of Eq. (F.37), vanishes identically. As we move away from $E=m+2 \omega_{1}$, the two sides start to differ, but we expect this difference to grow at least linearly in $E-\left(m+2 \omega_{1}\right) \propto 1 / B_{1}$.

The upshot is that particle-interchange symmetry leads to the following form for $\mathcal{K}_{\mathrm{df}, 3}^{s}$ :

$$
\mathcal{K}_{\mathrm{df}, 3}^{s}=\left(\begin{array}{ccc}
\mathcal{O}\left(\epsilon / B_{1}^{2}\right) & \mathcal{O}\left(\epsilon / B_{1}\right) & \mathcal{O}\left(\epsilon / B_{1}\right) \\
\mathcal{O}\left(\epsilon / B_{1}\right) & \mathcal{O}(\epsilon) & \mathcal{O}(\epsilon) \\
\mathcal{O}\left(\epsilon / B_{1}\right) & \mathcal{O}(\epsilon) & \mathcal{O}(\epsilon)
\end{array}\right)
$$

The top-left element is doubly suppressed because it involves a cancellation of the type just described for both left and right-hand indices. Combined with the result for $F_{3}^{s}$, Eq. (F.36), this implies that $\operatorname{det}\left(1+F_{3}^{s} \mathcal{K}_{\mathrm{df}, 3}^{s}\right)=1+\mathcal{O}(\epsilon)$. Thus there are no solutions to the quantization condition, and no unwanted states.2

\footnotetext{
${ }^{2}$ There is another source of suppression arising from particle-interchange symmetry, arising from the
} 
We now demonstrate Eq. (F.37). On the left-hand side the spectator momentum is $\vec{k}=(1,0,0)$, so the total momentum of the other two particles is $-\vec{k}$. By assumption (given our truncation) the amplitude in the CM frame of the other two particles is independent of angle. For one choice of angle the other two momenta are $\overrightarrow{0}$ and $-\vec{k}$ (since this gives the correct energy $E$ ). Thus the amplitude on the left-hand side of (F.37) is equal to the original $\mathcal{K}_{\mathrm{df}, 3}$ (with no superscript $s$ ) when the three incoming momenta are $\vec{k}, \overrightarrow{0}$ and $-\vec{k}$. By exactly the same argument, the amplitude on the right-hand side equals $\mathcal{K}_{\mathrm{df}, 3}$ for incoming momenta $-\vec{k}, \overrightarrow{0}$ and $\vec{k}$. But since $\mathcal{K}_{\mathrm{df}, 3}$ is symmetric under incoming particle exchange, the amplitudes on the two sides are equal.

endcaps $A$ and $A^{\prime}$ [see Eq. (5.244]). These have vanishing coupling to the symmetry-violating states when $E=m+2 \omega_{1}$. However, this alone would not be enough to remove these states from the spectrum if their energies were shifted slightly from the free-particle value. 


\section{VITA}

Max Hansen was born in Bozeman, MT to wonderful parents Taylor Hansen and Elizabeth Healy.

He welcomes your comments at mth28@uw. edu. 